\title{
MONOLITHIC INTEGRATED CERAMIC WAVEGUIDE FILTERS
}

\author{
MUHAMMAD YAMEEN SANDHU \\ Submitted in accordance with the requirements for the degree of \\ Doctor of Philosophy
}

The University of Leeds,

School of Electronic \& Electrical Engineering.

June 2014

This copy has been supplied on the understanding that it is copyright material and that no quotation from the thesis may be published without proper acknowledgement. 


\section{DECLARATION}

The candidate confirms that the work submitted is his own, except where work which has formed part of jointly-authored publications has been included. The contribution of the candidate and the other authors to this work has been explicitly indicated below. The candidate confirms that appropriate credit has been given within the thesis where reference has been made to the work of others.

The details of chapter 4 and 5 of the thesis are based on the following published paper:

[1] Ian C Hunter, Muhammad Y Sandhu, "Monolithic integrated ceramic waveguide filters," presented at IEEE MTT-S International Microwave Symposium, June 2014.

The candidate (Muhammad Yameen Sandhu) performed the experimental work and prepared the initial draft along with the graphical and tabular presentation, calculation and summarization of the paper.

The co-author (Prof. Ian Hunter) supervised the work, proof read the drafts and made suggestions and corrections to the draft paper.

(C) 2014 The University of Leeds and <Muhammad Yameen Sandhu> 


\begin{abstract}
Design techniques for a new class of monolithic integrated high permittivity ceramic rectangular waveguide microwave filters are presented in this thesis. These filters enable a size reduction of $50 \%$ as compared to air filled coaxial resonator filters with the same unloaded Q-factor. Initially, an integrated ceramic rectangular waveguide resonator structure is investigated. It consists of a metal plated high permittivity ceramic rectangular block with Q-factor comparable to transverse electromagnetic (TEM) coaxial resonator but in a much miniaturised volume with good out of band spurious performance. The three dimensional finite element method (FEM) electromagnetic solver HFSS ${ }^{\mathrm{TM}}$ is used to analyse the resonant modes, Q-factor and field patterns of the ceramic waveguide resonator.
\end{abstract}

High performance Chebyshev and generalised Chebyshev monolithic integrated ceramic rectangular waveguide filters are designed to meet the stringent electrical requirement for cellular radio base station. Inter-resonator couplings are achieved by placing various through and blind holes in the broad dimension of the waveguide. In the generalised chebyshev filter, both negative and positive cross couplings are introduced to achieve transmission zeros on both sides of the passband. Metal tuning screws are added to the generalised chebyshev design to correct any practical imperfections. The ceramic waveguide filters are excited through coaxial probes placed at the centre of the broad wall of the external resonators.

An integrated ceramic rectangular waveguide diplexer design is also presented to be used at mobile base station front end to replace an existing TEM diplexer without degrading electrical performance in a much miniaturised volume. The both filters and the common junction of the diplexer consist of single metal coated ceramic block with various blind and through holes to realize a complex coupling scheme.

Finally a low pass ceramic corrugated waveguide filter design is presented to be used along with diplexer at cellular base station to achieve very wide spurious free out of band bandwidth. The miniaturisation techniques discussed in this thesis will provide overall cost reduction for cellular communication systems requiring low loss narrowband bandpass filters. 


\section{ACKNOWLEDGEMENT}

First and foremost, I would like to thank Almighty Allah for the countless blessings $\mathrm{He}$ has bestowed upon me. My deep and sincere gratitude to Prof. Ian Hunter for his much needed kind support and constructive guidance both in an academic and personal capacity. I would like to thank Radio Design Limited to let me use their facilities to optimise and measure the filters. Thanks to Martin Gostling, Richard Parry, Michael Guess, Phil Sleigh and all others at Radio Design for giving their valuable suggestions to optimise the filters. I deeply appreciate Shokat Khokhar for correcting grammatical and editorial mistakes in my thesis.

I am very grateful to management of my sponsor Sukkur Institute of Business Administration for providing me the opportunity to pursue my studies. Sukkur IBA administration continued to support me financially despite having critical budget shortage.

I am deeply indebted to my parents, family and loved ones for their patience, sacrifice, valuable help, kind support, unconditional love and encouragement throughout my studies. My gratitude to my parents is more than that can be expressed in words.

Last but not least, I am much thankful to my colleagues and friends for their wonderful company and making my stay full of life. 


\section{CONTENTS}

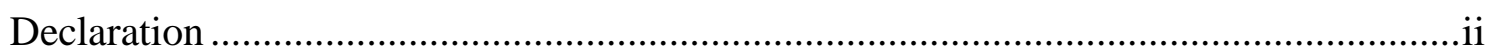

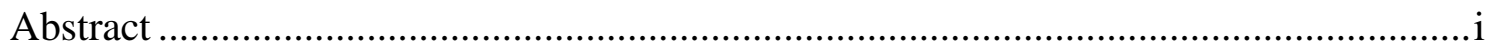

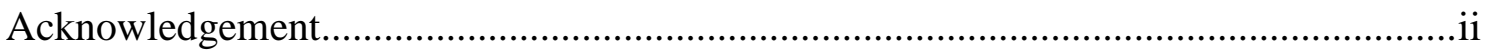

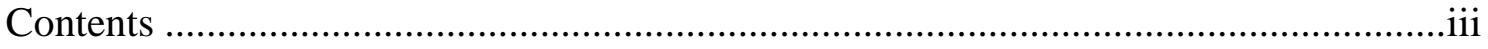

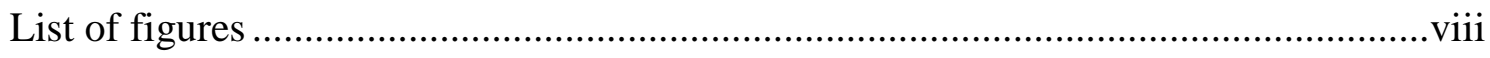

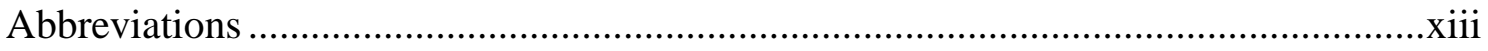

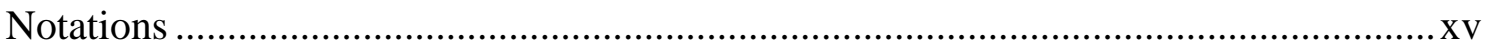

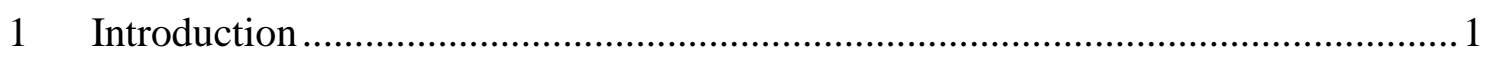

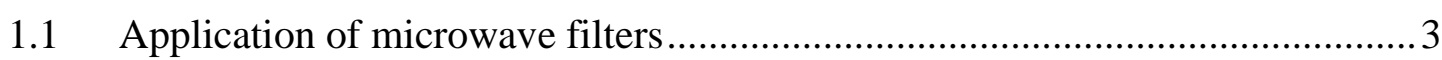

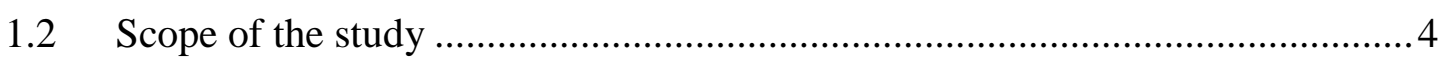

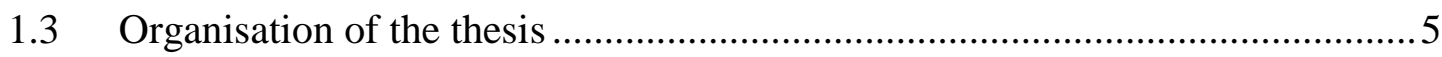

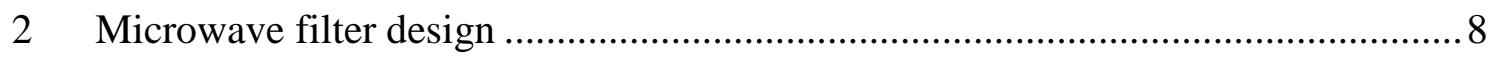

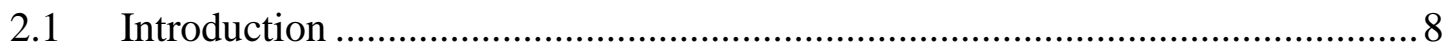

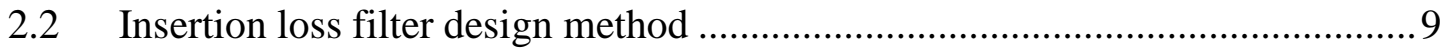

2.2.1 Low pass filter prototype …............................................................. 11

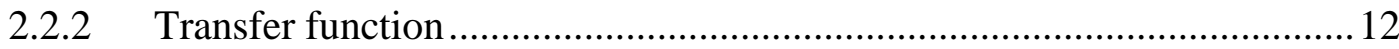

2.2.3 Frequency and impedance transformation .......................................... 17

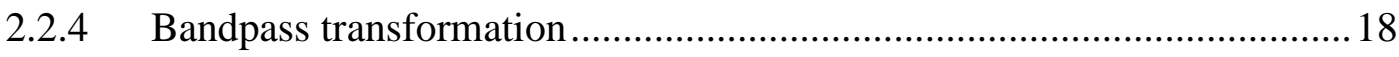

2.3 Implementation of microwave filters ….................................................. 19

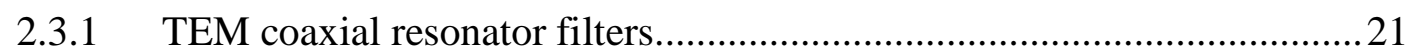

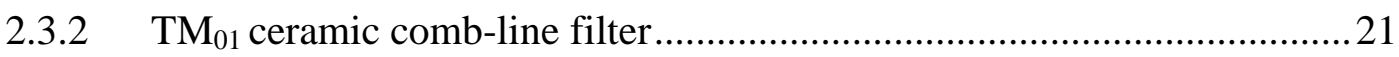

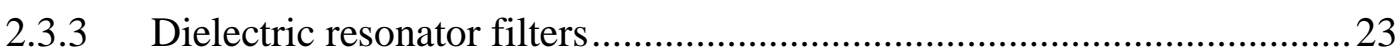




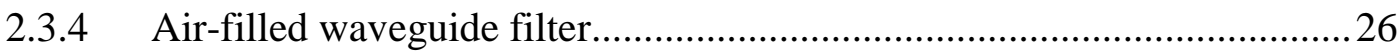

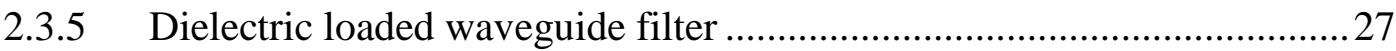

2.3.6 Integrated ceramic waveguide filter..................................................2 29

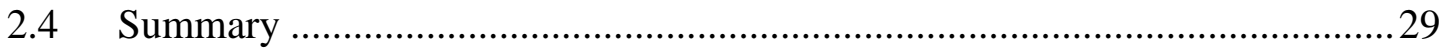

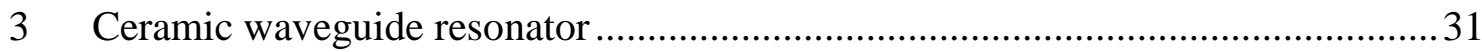

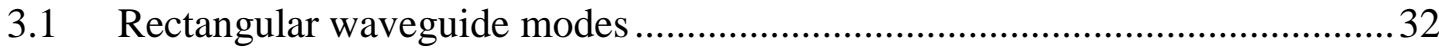

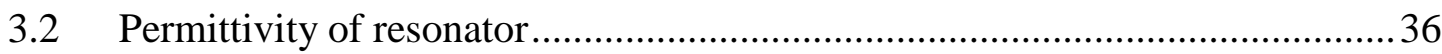

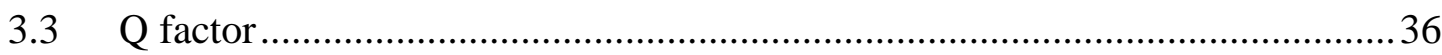

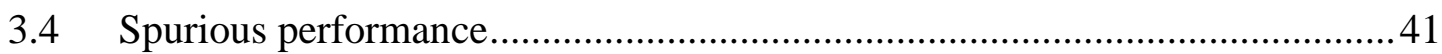

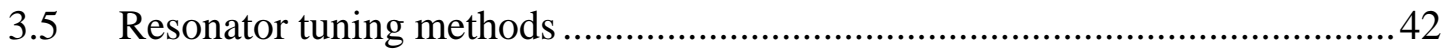

3.6 High Frequency Structure Simulator $\left(\mathrm{HFSS}^{\mathrm{TM}}\right)$....................................... 44

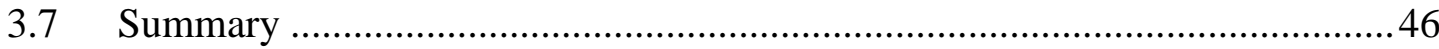

4 Chebyshev ceramic waveguide bandpass filter.................................................. 48

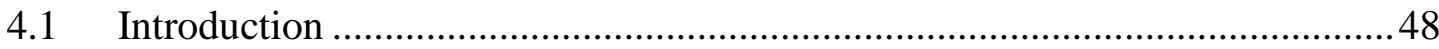

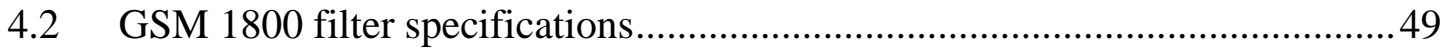

4.3 Lumped element Chebyshev filter design ................................................... 49

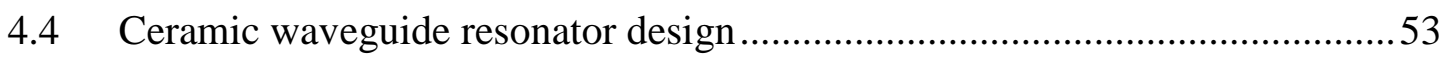

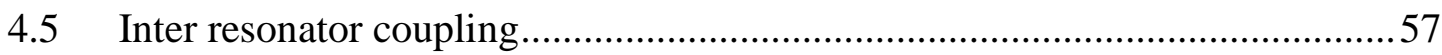

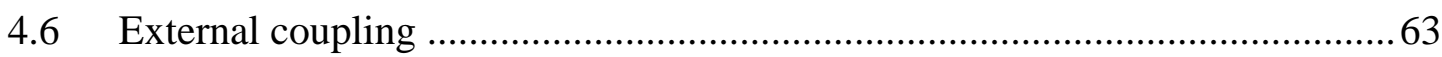

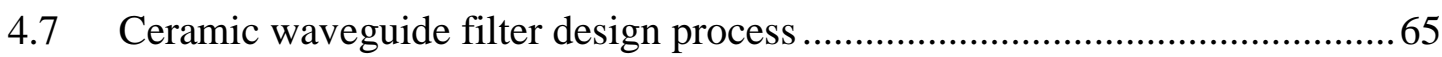

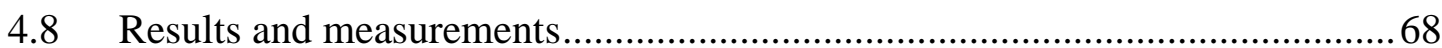

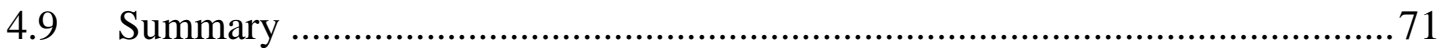

5 Generalized Chebyshev ceramic rectangular waveguide bandpass filter .............. 72

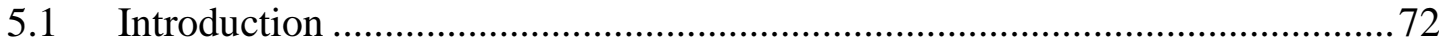

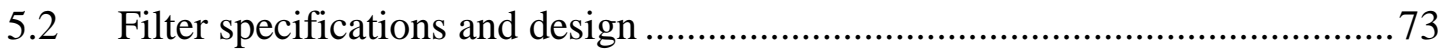

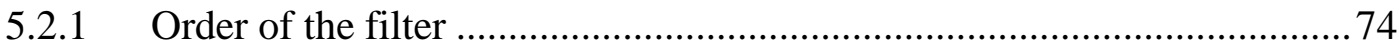




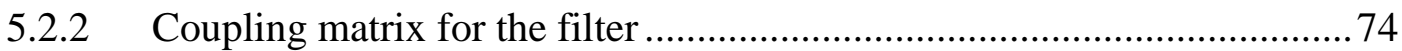

5.2.3 Lumped element impedance inverters ................................................76

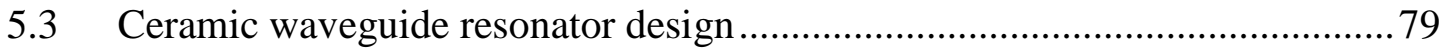

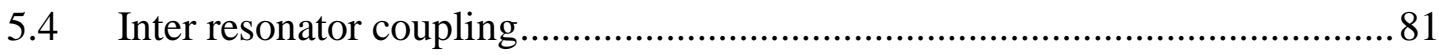

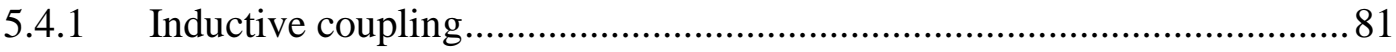

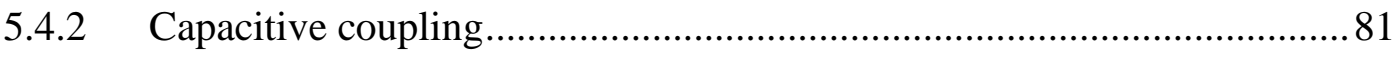

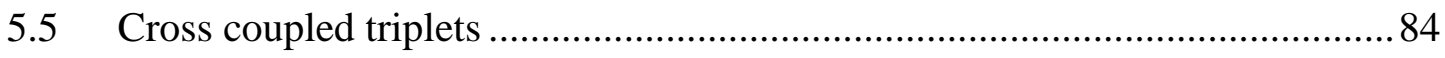

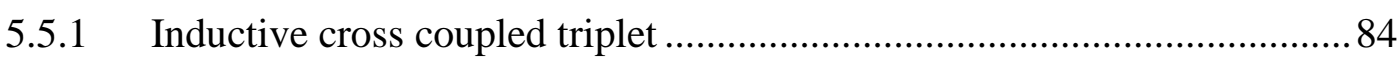

5.5.2 Capacitive cross coupled triplet ....................................................... 86

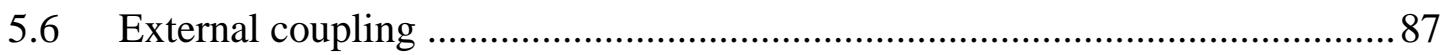

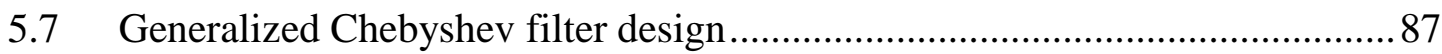

5.7.1 Filter with transmission zero above pass band..................................... 88

5.7.2 Filter with transmission zero below pass band .................................... 90

5.7.3 Filter with transmission zeros above and below pass band......................92

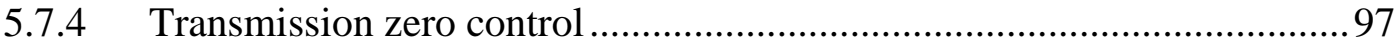

5.7.5 Generalized Chebyshev filter with tuning screws.................................. 98

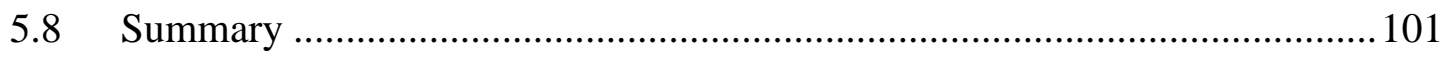

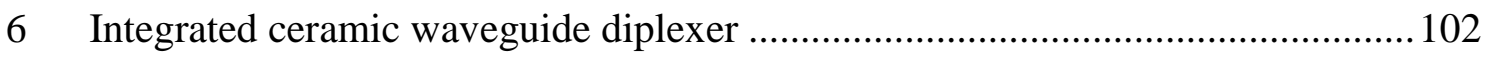

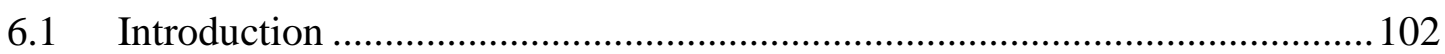

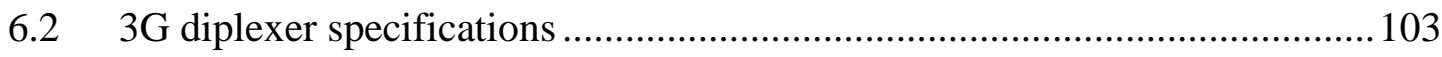

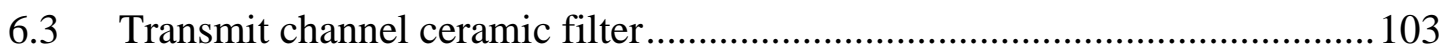

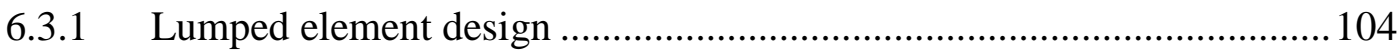

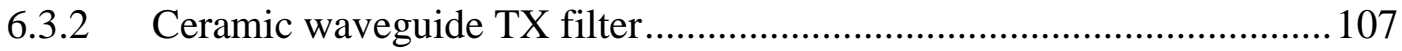

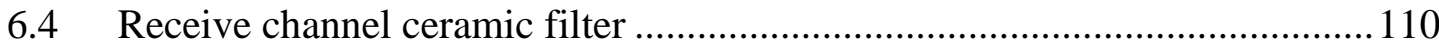

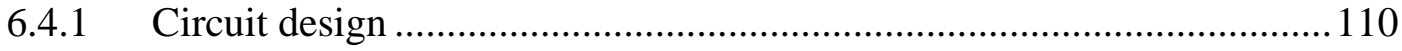

6.4.2 Ceramic waveguide realisation .................................................... 112 


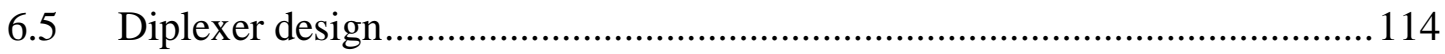

6.5.1 Circuit design ....................................................................................... 115

6.5.2 Ceramic waveguide realisation of diplexer..............................................118

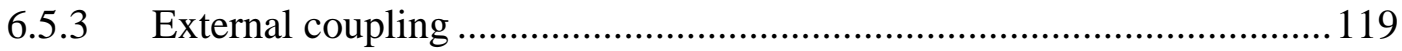

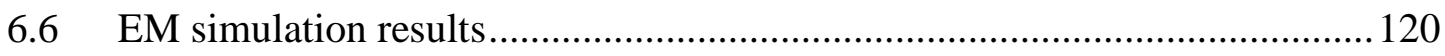

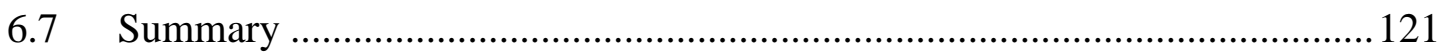

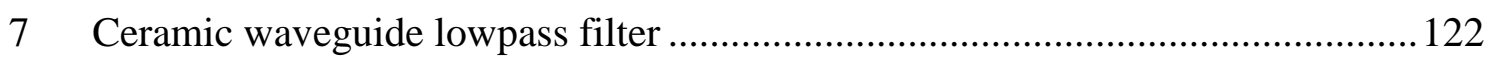

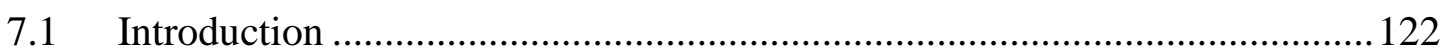

7.2 Image parameter filter design method ............................................................ 124

7.3 Corrugated waveguide LPF design procedure …………………………….... 128

$7.4 \quad$ Simulated Results for GSM1800................................................................131

7.4.1 Corrugated waveguide lowpass filter....................................................... 131

7.4.2 Improved spur corrugated waveguide LPF ...........................................134

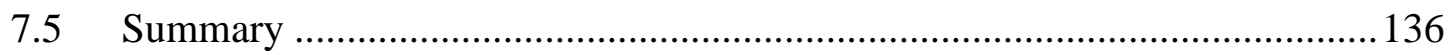

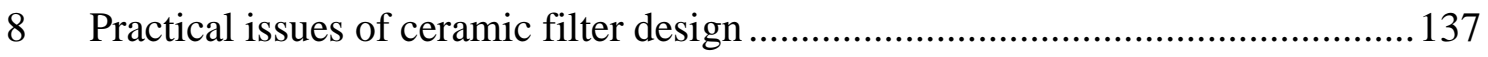

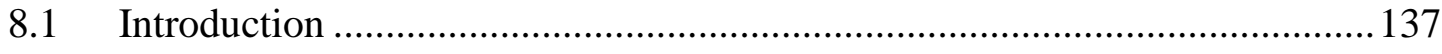

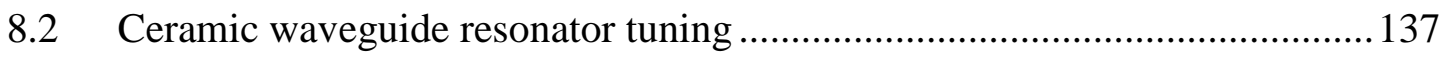

8.2.1 Tuning with metal screw ...................................................................... 138

8.2.2 Tuning with ceramic screw ................................................................... 140

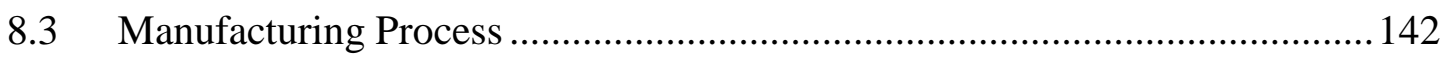

$8.4 \quad$ Temperature Effects ................................................................................... 142

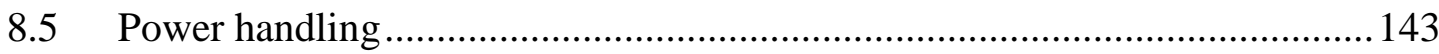

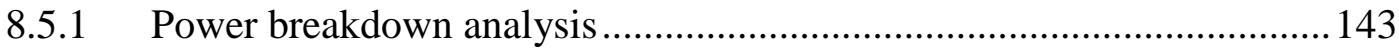

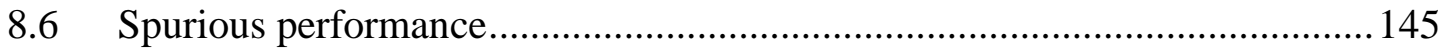

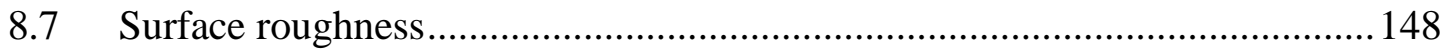

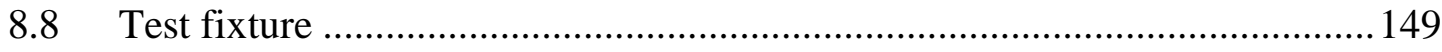




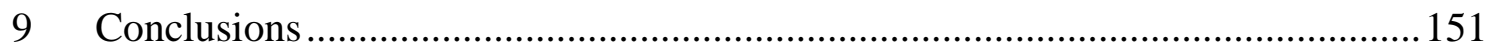

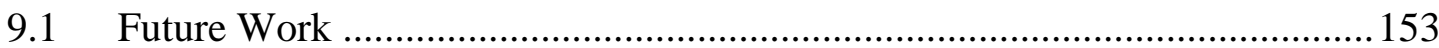

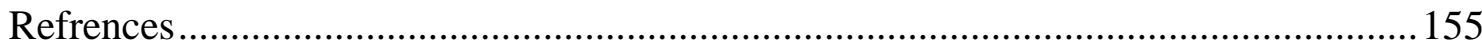




\section{LIST OF FIGURES}

Figure 1.1: Block diagram of RF front end of cellular base station...............................

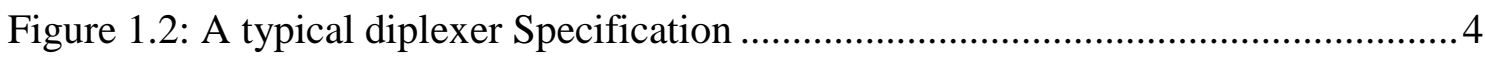

Figure 2.1: Design steps of insertion loss method .................................................. 10

Figure 2.2: Normalized low pass prototype ladder network ....................................... 11

Figure 2.3: Impedance inverter terminated with a load $\mathrm{Z}_{\mathrm{L} . \ldots \ldots \ldots \ldots \ldots \ldots \ldots \ldots \ldots \ldots \ldots \ldots \ldots \ldots \ldots \ldots \ldots \ldots \ldots \ldots \ldots \ldots \ldots \ldots \ldots} 12$

Figure 2.4: Inverter coupled generalized low pass prototype filter ............................... 12

Figure 2.5 : Butterworth low pass filter response .................................................... 14

Figure 2.6: Chebyshev low pass filter response .......................................................... 16

Figure 2.7: Generalised Chebyshev low pass filter response........................................ 17

Figure 2.8: Bandpass impedance and frequency scaled filter ...................................... 19

Figure 2.9: TEM coaxial resonator (a) Top view (b) Side view (c) Equivalent circuit .. 21 Figure 2.10: $\mathrm{TM}_{01}$ Ceramic comb-line resonator (a) Top view (b) Side view (c)

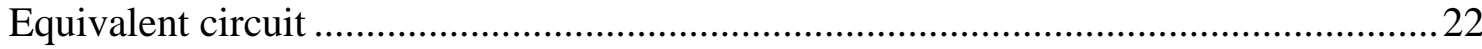

Figure 2.11: $\mathrm{TE}_{01 \delta}$ Dielectric resonator filter (Side view) .............................................2 23

Figure 2.12: Air filled rectangular waveguide cavity filter (i) Top view (ii) Side view. 27

Figure 2.13: Dielectric loaded waveguide filter........................................................... 28

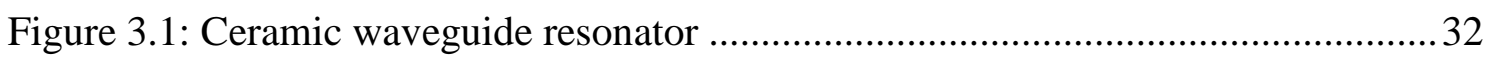

Figure 3.2: Electric and magnetic field pattern of rectangular waveguide resonator ......33

Figure 3.3: The mode chart for rectangular waveguide resonator with $\mathrm{B} / \mathrm{A}=0.5[20] \ldots 35$

Figure 3.4: Chart for estimating the unloaded $\mathrm{Q}$ of $\mathrm{TE}_{101}$ mode rectangular waveguide

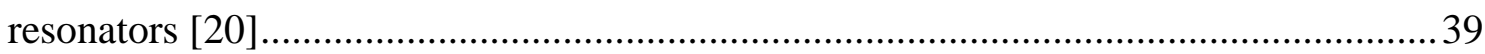

Figure 3.5: Coaxial and ceramic waveguide resonator Q-factor versus volume

Figure 3.6: Spurious resonance variation with respect to change in the height of ceramic waveguide resonator having $(\mathrm{a}=\mathrm{d}=18 \mathrm{~mm})$

Figure 3.7: Q-factor variation with respect to change in the height of a ceramic waveguide resonator having $(\mathrm{a}=\mathrm{d}=18 \mathrm{~mm})$ .42

Figure 3.8: Metal tuner (a) Top view (b) Side view (c) Equivalent circuit ....................43

Figure 3.9: Ceramic waveguide resonator tuning with metal screw

Figure 3.10: Mesh with tetrahedral elements to approximate the shape of a 3D object.45

Figure 3.11: Finite element method analysis of ceramic waveguide with different mesh resolutions (a) $0.6 \lambda$ mesh (b) $0.2 \lambda$ mesh 46 
Figure 4.1: Impedance inverter equivalent circuit

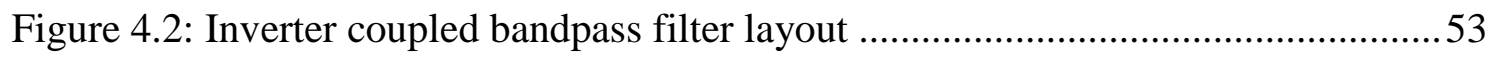

Figure 4.3: Inverter coupled lumped element bandpass filter response.

Figure 4.4: E-field distribution of ceramic waveguide resonator (Magnitude \& Vector)

Figure 4.5: H-field distribution of ceramic waveguide resonator (Magnitude \& Vector)

Figure 4.6: Through hole in ceramic (i) Top view (ii) Side view (iii) Equivalent circuit 57

Figure 4.7 : Normalized susceptance for two posts in a waveguide [58] ......................58

Figure 4.8: Shunt inductive iris embedded in a waveguide section

Figure 4.9: \% Fractional coupling bandwidth with different hole distance from sidewall 63

Figure 4.10: An equivalent circuit of six pole rectangular ceramic WG filter 68

Figure 4.11: Fabricated six pole Chebyshev ceramic Waveguide filter .70

Figure 4.12: $1842.5 \mathrm{MHz}$ ceramic Waveguide band pass filter measured \& simulated response

Figure 4.13: $1842 \mathrm{MHz}$ ceramic Waveguide filter passband insertion loss (measured vs HFSS $^{\mathrm{TM}}$ ) .71

Figure 5.1: Coupling matrix of generalised Chebyshev bandpass filter..... 75

Figure 5.2: K-inverter and its equivalent lumped element circuit 77

Figure 5.3: Inverter coupled generalized Chebyshev bandpass filter circuit design....... 78

Figure 5.4: Simulated lumped element generalized Chebyshev bandpass filter response

Figure 5.5: Waveguide capacitive impedance inverter (i) Side view (ii) Top view (iii) Equivalent circuit

Figure 5.6: Ceramic waveguide inductive triplet (i) Top view (ii) Coupling scheme (iii)

Side view.....

Figure 5.7: High side transmission zero control by varying inductive cross coupling ... 85 Figure 5.8: Ceramic waveguide capacitive triplet (i) Top view (ii) Side view (iii) Coupling scheme. 86

Figure 5.9: Low side transmission zero control by varying capacitive cross coupling .. 87 Figure 5.10: Coupling matrix of generalised Chebyshev filter with one cross coupling 88 
Figure 5.11: Generalized Chebyshev filter configuration with an inductive triplet 89

Figure 5.12: Generalized Chebyshev ceramic waveguide filter layout with an inductive triplet (i) Top view (ii) Side view

Figure 5.13: A generalised Chebyshev ceramic waveguide filter with Transmission zero above passband ( $\mathrm{HFSS}^{\mathrm{TM}}$ simulation)

Figure 5.14: Coupling matrix for a generalised Chebyshev filter with negative cross coupling .90

Figure 5.15: Generalized Chebyshev filter configuration with a capacitive triplet 91

Figure 5.16: Generalized Chebyshev ceramic waveguide filter layout with capacitive cross coupled triplet (i) Top view (ii) Side view.

Figure 5.17: Generalised Chebyshev ceramic waveguide bandpass filter response with a transmission zero below passband (HFSS ${ }^{\mathrm{TM}}$ Simulation) 92

Figure 5.18: Coupling matrix of generalised Chebyshev BPF with a capacitive and an inductive cross coupled triplet .93

Figure 5.19: Generalized Chebyshev filter configuration with a capacitive and an inductive cross coupled triplet

Figure 5.20: Ceramic waveguide generalised Chebyshev bandpass filter drawing

Figure 5.21: Simulated RX filter with Transmission zero below and above passband $\left(\mathrm{HFSS}^{\mathrm{TM}}\right)$ 94

Figure 5.22: Fabricated ceramic waveguide RX filter 95

Figure 5.23 : Measured and HFSS ${ }^{\mathrm{TM}}$ simulated response 95

Figure 5.24 : Measured and EM simulated response with side resonator radii included 96

Figure 5.25: RX filter with transmission zero near pass-band. 98

Figure 5.26: Ceramic waveguide filter with tuning screws (i) bottom view (ii) Side view

Figure 5.27: Simulated generalised Chebyshev ceramic WG BPF with tuning screws $\left(\right.$ HFSS $\left.^{\mathrm{TM}}\right)$

Figure 5.28 : Fabricated generalised Chebyshev filter with tuning screws 100

Figure 5.29 : Simulated and measured response of generalised Chebyshev filter with tuning screws

Figure 6.1: Capacitive coupled normalised low pass prototype filter.

Figure 6.2: Capacitive coupled Chebyshev bandpass filter circuit diagram 107

Figure 6.3: Lumped element Chebyshev bandpass filter response (Circuit simulation) 
Figure 6.4: Inter resonator coupling bandwidths variation with respect to hole offset $\left(\mathrm{X}_{1}\right)$ from side walls of the waveguide

Figure 6.5: TX Chebyshev ceramic waveguide filter layout (i) Top view (ii) Side view

Figure 6.6: Simulated TX filter response $\left(\mathrm{HFSS}^{\mathrm{TM}}\right)$.

Figure 6.7: Coupling matrix for Receive channel filter 111

Figure 6.8: Simulated RX filter circuit design response

Figure 6.9: Generalized Chebyshev RX ceramic waveguide filter layout

Figure 6.10: Simulated generalized Chebyshev RX filter response (HFSS ${ }^{\mathrm{TM}}$ )

Figure 6.11: Generalized Chebyshev RX filter pass band insertion loss

Figure 6.12: Diplexer configuration.

Figure 6.13: Inverter-coupled diplexer circuit design

Figure 6.14: Simulated diplexer circuit design response

Figure 6.15: Passband insertion loss of RX and TX filter

Figure 6.16: Ceramic rectangular waveguide diplexer (i) Top view (ii) Side view

Figure 6.17: Simulated ceramic waveguide diplexer response $\left(\mathrm{HFSS}^{\mathrm{TM}}\right)$

Figure 7.1: Corrugated waveguide low pass filter layout (i) Side View (ii) Top view. 124

Figure 7.2: Two port network terminated in its image impedance 125

Figure 7.3: (a) Constant K-section (b) m-derived half section of low pass filter 126

Figure 7.4: Low pass filter circuit diagram

Figure 7.5: Simulated low pass filter response

Figure 7.6: Corrugated waveguide half section 128

Figure 7.7: Corrugated waveguide low pass filter

Figure 7.8: Simulated corrugated ceramic waveguide low pass filter response (HFSS ${ }^{\mathrm{TM}}$ )

Figure 7.9: Simulated passband IL of ceramic corrugated waveguide filter $\left(\mathrm{HFSS}^{\mathrm{TM}}\right) 133$

Figure 7.10: Change in cut-off, attenuation pole and spurious resonance frequencies with the height of the high impedance section of corrugated LPF

Figure 7.11: Simulated corrugated ceramic waveguide filter response with two different heights of high impedance section $\left(\mathrm{HFSS}^{\mathrm{TM}}\right.$ )

Figure: 8.1: Simulated resonance frequency tuning with metal tuning screw of different diameter. 
Figure: 8.2: Simulated tuning screw length effect on unloaded Q of the resonator...... 139

Figure: 8.3: Ceramic resonator with ceramic tuner (a) Top view (b) Side view (c)

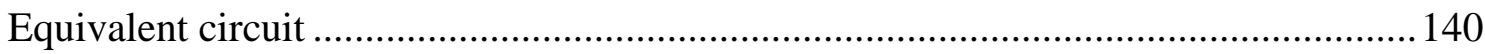

Figure 8.4: Simulated resonance frequency tuning with ceramic tuner...................... 141

Figure: 8.5: Simulated Ceramic tuner effect on unloaded Q-factor of resonator.......... 142

Figure 8.6: Simulated TX filter E-field distribution .................................................. 144

Figure 8.7: Simulated wideband response of TX filter .............................................. 145

Figure 8.8: Simulated broadband response of RX filter .......................................... 146

Figure 8.9: Simulated ceramic rectangular waveguide fundamental and first spurious frequency variation by placing the metal coated hole at the centre of broad wall of it.147 Figure 8.10: Simulated ceramic rectangular waveguide unloaded Q-factor variation by placing the metal coated hole at the centre of broad wall of it. ................................. 147

Figure 8.11: Simulated unloaded Q degradation with surface roughness.................... 149

Figure 8.12: Fabricated RX filter with I/O pins ....................................................... 150

Figure 8.13: Test fixture with ceramic filter (a) Top view (b) Side view ..................... 150 


\section{ABBREVIATIONS}

\begin{tabular}{|c|c|}
\hline $\mathrm{BPF}$ & Bandpass filter \\
\hline BTS & Base transceiver station \\
\hline BW & Bandwidth \\
\hline DR & Dielectric resonator \\
\hline $\mathrm{GHz}$ & Gigahertz \\
\hline GSM & Global system for mobile communication \\
\hline HFSS & High frequency structure simulator \\
\hline HTS & High temperature super conducting \\
\hline $\mathrm{I} / \mathrm{P}$ & Input \\
\hline IL & Insertion loss \\
\hline LPF & Lowpass filter \\
\hline LTCC & Low temperature co-fired ceramic \\
\hline $\mathrm{MHz}$ & Megahertz \\
\hline PCS & Personnel communication system \\
\hline PEC & Perfect electric conductor \\
\hline PLR & Power loss ratio \\
\hline RL & Return loss \\
\hline $\mathrm{TZ}$ & Transmission zero \\
\hline UMTS & Universal mobile telecommunication system \\
\hline WG & Waveguide \\
\hline EM & Electromagnetic \\
\hline FEM & Finite element method \\
\hline $\mathrm{RF}$ & Radio frequency \\
\hline $\mathrm{RX}$ & Receive \\
\hline $\mathrm{TE}$ & Transverse electric \\
\hline TEM & Transverse electromagnetic \\
\hline TM & Transverse magnetic \\
\hline TX & Transmit \\
\hline
\end{tabular}


$\mathrm{KHz}$

$\mathrm{MV} / \mathrm{m}$

KW
Kilohertz

Megavolt per metre

Kilowatts 


\section{NOTATIONS}

\begin{tabular}{|c|c|}
\hline$A_{A B C D}$ & A parameter of transfer matrix \\
\hline$A_{\text {Area }}$ & Area of coupling blind hole \\
\hline$B_{A B C D}$ & B parameter of transfer matrix \\
\hline$B_{c 1}$ & Series susceptance \\
\hline$B_{c 2}$ & Shunt susceptance \\
\hline$B_{r, r+1}$ & Susceptance of coupling section between resonator $r$ and $r+1$ \\
\hline$C_{A B C D}$ & C parameter of transfer matrix \\
\hline$C_{s}^{\prime}$ & Impedance scaled capacitor in series branch \\
\hline$C_{s}^{\prime}$ & Impedance scaled capacitor in parallel branch \\
\hline$D_{A B C D}$ & D parameter of transfer matrix \\
\hline$D_{c}$ & Coaxial probe outer conductor diameter \\
\hline$E_{\text {peak }}$ & Peak electric field \\
\hline$E_{x}$ & E-field component in $\mathrm{x}$ direction \\
\hline$E_{y}$ & E-field component in y direction \\
\hline$E_{z}$ & E-field component in $\mathrm{z}$ direction \\
\hline$F_{B W}$ & Fractional bandwidth \\
\hline$F_{n}(\omega)$ & Filtering function \\
\hline$H_{0}$ & Magnitude of H-field \\
\hline$H_{x}$ & $\mathrm{H}$-field component in $\mathrm{x}$ direction \\
\hline$H_{y}$ & $\mathrm{H}$-field component in y direction \\
\hline$H_{z}$ & $\mathrm{H}$-field component in $\mathrm{z}$ direction \\
\hline$K_{r, r+1}$ & Coupling coefficient between resonator $r$ and $r+1$ \\
\hline$L_{A}$ & Stopband attenuation \\
\hline$L_{R}(\omega)$ & Return loss \\
\hline$L_{p}^{\prime}$ & Impedance scaled inductor in parallel branch \\
\hline$L_{S}^{\prime}$ & Impedance scaled inductor in series branch \\
\hline$P_{L R}$ & Power loss ratio \\
\hline$P_{c}$ & Power dissipation due to conductor loss \\
\hline
\end{tabular}




\begin{tabular}{|c|c|}
\hline$P_{d}$ & Power dissipation due to dielectric loss \\
\hline$P_{r}$ & Power dissipation due to radiation loss \\
\hline$Q_{u}$ & Unloaded quality factor \\
\hline$Q_{c}$ & Conduction loss \\
\hline$Q_{d}$ & Dielectric loss \\
\hline$Q_{r}$ & Radiation loss \\
\hline$T_{n}(\omega)$ & Chebyshev function \\
\hline$Y_{I}$ & Normalized image admittance \\
\hline$Y_{o c}$ & Open circuit admittance of half section of corrugated filter \\
\hline$Y_{s c}$ & Short circuit admittance of half section of corrugated filter \\
\hline$Z_{L}$ & Load Impedance \\
\hline$Z_{T E}$ & Impedance of rectangular waveguide in TE mode \\
\hline$Z_{c}$ & Impedance of coaxial cable \\
\hline$Z_{\text {in }}$ & Input impedance \\
\hline$Z_{w}$ & Impedance of rectangular waveguide \\
\hline$b_{T}$ & Height of terminating section of corrugated waveguide filter \\
\hline$b_{W G}$ & Height of bigger height section of corrugated waveguide filter \\
\hline$b_{W G}^{\prime}$ & Height of smaller height section of corrugated filter \\
\hline$b_{o c}$ & Open circuit suceptance of half section of corrugated filter \\
\hline$b_{s c}$ & Short circuit suceptance of half section of corrugated filter \\
\hline$d_{h}$ & Diameter of coupling through hole \\
\hline$d_{c}$ & Coaxial probe inner conductor radius \\
\hline$d_{\text {separation }}$ & Distance between the plates of a parallel plate capacitor \\
\hline$d_{y}$ & Input/output probe distance from shorted end \\
\hline$f_{0 R X}$ & Centre frequency of receive filter \\
\hline$f_{1}$ & Cuttoff frequency of waveguide low pass filter \\
\hline$f_{2}$ & Lower cuttoff frequency of next higher order mode of a LPF \\
\hline$f_{3}$ & Upper cuttoff frequency of next higher order mode of a LPF \\
\hline$f_{c}$ & Cuttoff frequency of waveguide \\
\hline$f_{\text {in }}$ & Attenuation pole frequency of waveguide \\
\hline$k_{1}$ & Ripple level in the passband \\
\hline$l_{W G}$ & Length of bigger height section of corrugated filter \\
\hline
\end{tabular}




\begin{tabular}{|c|c|}
\hline$l_{W G}^{\prime}$ & Length of smaller height section of corrugated filter \\
\hline$l_{i l}$ & Initial length \\
\hline$m_{L P F}$ & $\mathrm{~m}$ parameter of lowpass filter \\
\hline$p_{L}$ & Load power \\
\hline$p_{\text {in }}$ & Input power \\
\hline$r_{p}$ & Radius of input/output coupling probe \\
\hline$v_{g}$ & Group velocity \\
\hline$\alpha_{c}$ & Conductor loss \\
\hline$\alpha_{l}$ & Linear coefficient of thermal expansion \\
\hline$\varepsilon_{0}$ & Vacuum Permittivity \\
\hline$\varepsilon_{r}$ & Relative permittivity \\
\hline$\eta_{0}$ & Characteristic wave impedance \\
\hline$\theta^{\prime}$ & Electrical length of lower height section of corrugated filter \\
\hline$\lambda_{c}$ & Waveguide cut-off wavelength \\
\hline$\lambda_{d}$ & Wavelength in dielectric medium \\
\hline$\lambda_{g}$ & Guide wavelength \\
\hline$\lambda_{g 0}$ & Guide wavelength at resonance frequency \\
\hline$\omega_{0}$ & Angular resonant frequency \\
\hline$\omega_{c}$ & Angular cuttoff frequency \\
\hline$\omega_{p}$ & Passband frequency \\
\hline$\omega_{s}$ & Stopband frequency \\
\hline$\Delta$ & Surface roughness \\
\hline$\Delta l_{l}$ & Change in length \\
\hline$B$ & Susceptance \\
\hline$g_{i}$ & Lowpass prototype element values \\
\hline$h$ & Input/output coupling probe depth inside waveguide \\
\hline$k_{e}$ & Normalized external coupling coefficient \\
\hline$K_{e}$ & External coupling bandwidth \\
\hline$L_{e}$ & Inductor used to form external coupling impedance inverter \\
\hline$r$ & Radius of blind/ through holes in ceramic waveguide \\
\hline$x_{1}$ & Distance of coupling hole from sidewall of waveguide \\
\hline$Y$ & Admittance \\
\hline
\end{tabular}




\begin{tabular}{|c|c|}
\hline$\Omega$ & Normalized cuttoff frequency of lowpass prototype \\
\hline C & Capacitor \\
\hline$K$ & Characteristic impedance \\
\hline$L$ & Inductor \\
\hline$N$ & Order of the filter \\
\hline$S$ & Ratio of stopband to passband bandwidth \\
\hline$W$ & Total stored energy of the structure \\
\hline$Z_{0}$ & Impedance \\
\hline$a$ & Ceramic waveguide resonator width \\
\hline$b$ & Ceramic waveguide resonator height \\
\hline$d$ & Ceramic waveguide length \\
\hline$f$ & Frequency \\
\hline$l$ & $\begin{array}{l}\text { Number of half wave variations of E-field along width of } \\
\text { waveguide resonator }\end{array}$ \\
\hline$m$ & $\begin{array}{l}\text { Number of half wave variations of E-field along height of } \\
\text { waveguide resonator }\end{array}$ \\
\hline$n$ & $\begin{array}{l}\text { Number of half wave variations of E-field along length of } \\
\text { waveguide resonator }\end{array}$ \\
\hline ppm $/{ }^{0} \mathrm{C}$ & Parts per million per centigrade \\
\hline $\tan \delta$ & Dielectric loss tangent \\
\hline$v$ & Speed of light \\
\hline$\nabla$ & Ratio of centre frequency to bandwidth of a filter \\
\hline$\beta$ & Imaginary part of propagation constant \\
\hline$\gamma$ & Propagation constant \\
\hline$\delta$ & Skin depth of the conductor \\
\hline$\varepsilon$ & Passband ripple constant \\
\hline$\theta$ & Electrical length of transmission line \\
\hline$\lambda$ & Wavelength \\
\hline$\rho$ & Resistivity of the material \\
\hline$\sigma$ & Conductivity of the material \\
\hline$\tau$ & $\begin{array}{l}\text { Ratio of height of high impedance section to lower } \\
\text { impedance section in a corrugated waveguide filter }\end{array}$ \\
\hline
\end{tabular}


Phase length 


\section{INTRODUCTION}

Microwaves are the part of electromagnetic spectrum ranging from $300 \mathrm{MHz}$ to $30 \mathrm{GHz}$ with the wavelength from $1 \mathrm{~m}$ down to $1 \mathrm{~cm}$. Microwave filter technology is used in Radars, satellites and all other cellular communication systems [1]. Due to advent of smart phones and tablets, data traffic is increasing very rapidly. Presently, cellular infrastructure is in its development phase to support this rapid flood of data traffic initiated by smartphones and tablet computers. Conventional cellular base stations have their transceivers at the base of mast and they are connected to the antennas at the top of the mast through lengthy coaxial cables. Thus, a significant amount of signal is attenuated due to large distances between cellular transceiver and antenna. To avoid this amount of energy loss, transceiver needs to be placed at the top of the mast along with antenna. Therefore, miniaturized cellular components are required with lower mass and smaller volume without compromising the electrical performance. Moreover, the RF front end is analog and requires significant changes compatible with evolving digital signal processing techniques. A timeline of development in the area of RF and microwave filters is listed in [2]. The major part of the cellular base station transceiver consisting of filters with significant volume and mass is to rectify the desired electromagnetic signals from unwanted interferences. Thus, for achieving the ideal base station configuration, the biggest challenge is the size and mass reduction of microwave filters without compromising on its electrical performance. Miniaturization of RF microwave filters is the core objective of this research. RF microwave filters are analog electrical devices which are used to separate desired signal frequency band from undesired interference band as well as to isolate transmitted signals from received signals. These devices require stringent electrical performance specifications for transmission and reception. Microwave filter comprises several resonant sections 
aligned together to form required transfer function of the filter. The amount of energy dissipated in the resonant sections while passing through the filter is known as insertion loss of the filter. Total number of resonant sections and their configuration determine the order of filter and its selectivity. Conventional RF base stations use air filled coaxial comb-line cavities in order to implement filters. Air-filled coaxial comb-line resonators are simple structures having nominal Q factor and good spurious performance but they are not compatible with future miniaturized communication systems due to their large volumes. Ceramic materials offer very high Q resonators to design low loss ceramic filters. The wavelength of a wave passing through a dielectric material of permittivity $\varepsilon_{r}$ decreases by a factor of $\frac{1}{\sqrt{\varepsilon_{r}}}$. Therefore, the filter dimensions shrink significantly when high permittivity ceramic resonators are used. Although the insertion loss also increases in the same proportion but still for nominal Q values a significant volume reduction can be achieved. The aim of this thesis is to design low loss miniaturised monolithic integrated ceramic rectangular waveguide filters to be used in cellular base stations. The first objective of this thesis is to design a Chebyshev integrated ceramic waveguide band pass filter suitable for digital cellular system (DCS) uplink frequency band. This filter could be miniaturised alternative of conventional coaxial cavity filter. The second objective of this thesis is to design a more complex design of generalised Chebyshev ceramic waveguide filter with transmission zeros placed at the both sides of the pass band. The generalised Chebyshev ceramic waveguide filter design could meet the stringent commercial filter out of band specifications. The third objective of this research is to provide a complete miniaturized diplexer solution in the form of an integrated ceramic rectangular waveguide structure. The miniaturized integrated ceramic diplexer can be placed at the top of the mast along with the antenna system. Final objective of the thesis is to design a low pass corrugated integrated ceramic waveguide filter. The miniaturised corrugated ceramic waveguide filter can be used along with the ceramic band pass filter to provide wide spurious free stop band. The main focus of this work is to provide an introduction to size reduction of the microwave band pass filters using integrated ceramic waveguide resonators. This work can be further extended to realize much complex and advanced filter networks. 


\subsection{Application of microwave filters}

The frequency spectrum is becoming over crowded day by day and various communications systems are forced to work in adjacent frequency bands. Microwave filters are the key component of the mobile, terrestrial and radar communication systems used to pass wanted frequency spectrum and block unwanted noise signals. Rapidly growing mobile communication system requires more stringent filter specifications. Microwave filters are essential part of cellular base station front end [3]. They isolate the very weak signals received at the common port from the powerful transmitted signals by allowing desired frequency range through the filter network simultaneously rejecting all other frequency components. Usually low loss in the pass band of the filter is required with sharp transition from pass to stop band to avoid any interference from the adjacent channels. A wide spurious free bandwidth and symmetrical response is often required but in some cases asymmetric realizations are preferred. The selectivity of the filter is dictated by the Q-factor and number of resonators used in the filter. Figure 1.1 represents the block diagram of the cellular base station front end.

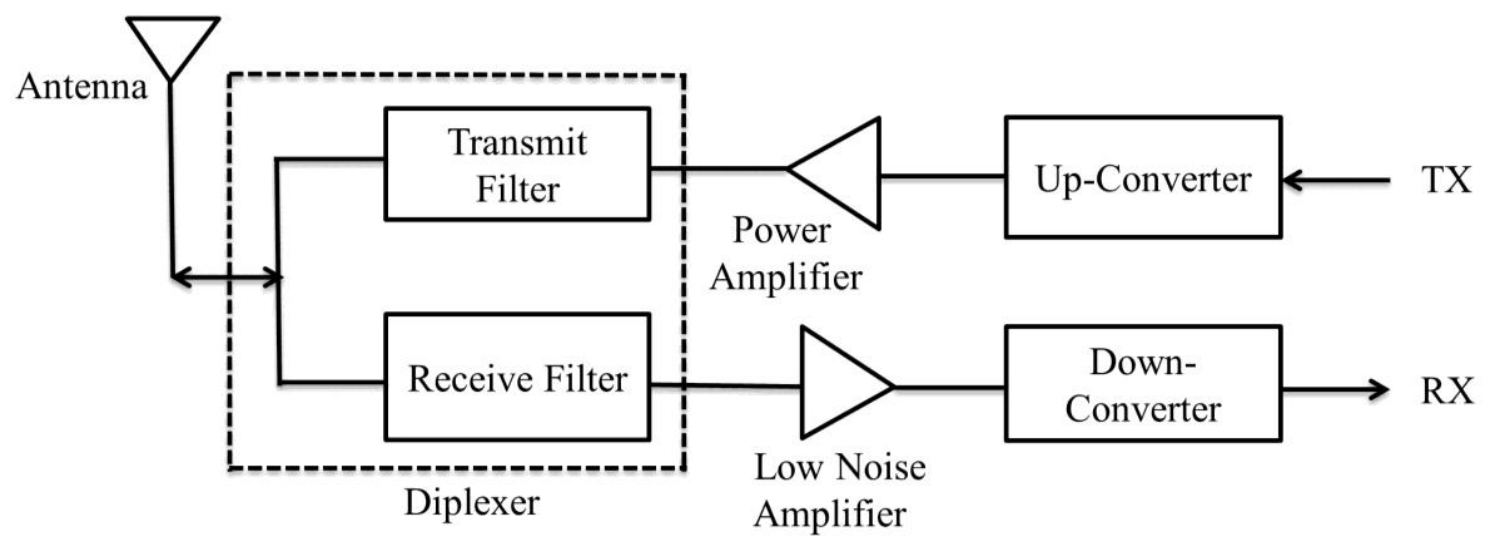

Figure 1.1: Block diagram of RF front end of cellular base station

Microwave filters are the key building blocks of diplexer and multiplexer devices used to combine or separate different frequency bands. Diplexers provide economical solution by allowing two different devices to communicate over the shared communication medium. A diplexer is a three port device which combines/separates 
frequency selective signals from TX and RX filter at the common port. Figure 1.2 shows the electrical response of a typical diplexer.

Band pass filters are the main part of almost every cellular communication transceiver to reduce the noise from the system. Microwave filters with different physical realisation are used in cellular base station and mobile handsets. Usually, filters with low pass band insertion loss, smaller volume and low cost are required. Monolithic integrated ceramic waveguide filter design presented in this thesis can be used in cellular base station front end which can save the volume of overall diplexer more than $50 \%$ as compared to conventional TEM resonator filters.

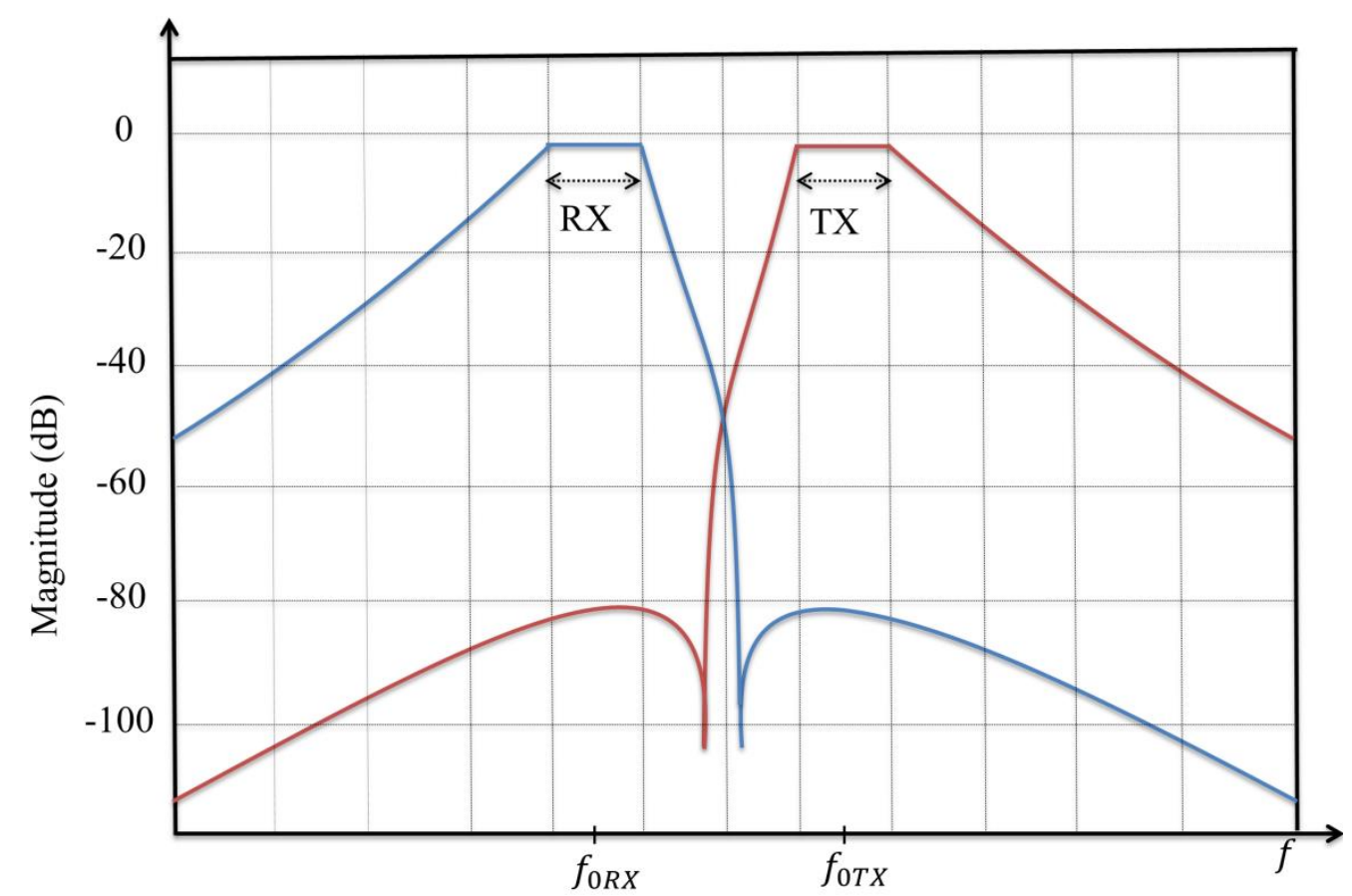

Figure 1.2: A typical diplexer Specification

\subsection{Scope of the study}

Miniaturization of the RF components has been of considerable interest recently. A wide variety of literature is published on single and multimode dielectric loaded cavity filters. Dielectric loaded cavity filters offer significant size reduction but still the demand to miniaturize the RF front end remains. Also the dielectric loaded cavity resonator suffers from poor spurious performance. In this study, a variety of integrated 
ceramic waveguide filters are designed, which offer more than $50 \%$ volume reduction than conventional air filled coaxial cavity resonator filters. Classical Chebyshev and generalized Chebyshev prototypes are used to realize the response of ceramic waveguide filter. The most of the research work presented here is focused on physical realisation of the ceramic waveguide filters using 3D EM simulation tool $\mathrm{HFSS}^{\mathrm{TM}}$. Firstly, a ceramic rectangular waveguide resonator section is investigated. It has been observed that ceramic waveguide resonator reduces the dimensions of an air filled waveguide cavity by a factor of $\frac{1}{\sqrt{\varepsilon_{r}}}$. It has the advantage of being integrated, therefore easy to manufacture. A simple Chebyshev and a more complex generalised Chebyshev ceramic waveguide filter design and fabrication is presented. Both of the designs have advantage of being monolithic integrated structures. In generalised Chebyshev realisation, transmission zeros are produced by allowing the cross coupled paths to nonadjacent resonators. Inter-resonator couplings are realized using various metal plated through and blind holes. Input and output couplings are achieved by a coaxial probe at the centre of the broad wall of the resonator. It makes it very easy to fabricate by taking a ceramic block and then adding details to it and finally the entire block is coated with a metal ink except input/output probe positions. Various coupling and resonator dimensions can be calculated theoretically and verified through an electromagnetic (EM) simulation. Post production tuning of resonators is achieved using metal tuning screws at the centre of each resonator. A monolithic integrated ceramic waveguide diplexer design is also presented, which could replace the waveguide or coaxial resonator filters used in current RF front end of cellular base stations. Finally, a low pass ceramic corrugated waveguide filter design is presented. This miniaturized device can be used along with the ceramic waveguide diplexer to provide the wide spurious free stop band bandwidth. Thus, the overall work presented here can be used in a commercial cellular base station front end to replace the existing diplexer section of the RF front end without degrading the electrical performance and in a much miniaturised space.

\subsection{Organisation of the thesis}

Firstly, this Chapter explains the need to miniaturize cellular front end. Applications of microwave filters in cellular communication system are explained here. Objectives of 
the research presented in this thesis are defined Chapter 1.

Chapter 2 gives a review of microwave filter design procedures. Insertion loss method of microwave filter design is explained in detail. Commonly used approximations for filter transfer functions are given. Commonly used physical implementations of microwave filter types used in cellular base stations are also reviewed.

Chapter 3 explains the ceramic waveguide resonator design. A comparison of ceramic waveguide resonator is done with conventional air filled coaxial comb-line resonator in terms of Q-factor verses volume. Resonator tuning methods, spurious performance and surface roughness effect on Q-factor is also discussed.

A six pole Chebyshev ceramic waveguide filter is designed in Chapter 4. The monolithic integrated ceramic waveguide filter structure uses metal coated through holes for inter resonator coupling. External couplings are achieved by coaxial probes. Details about coaxial to waveguide transitions are also given. A step by step design procedure for ceramic waveguide filter is explained. Tuning methods to introduce post production tuning is explained. Simulated and fabricated results for a $6^{\text {th }}$ order Chebyshev filter are presented and explained.

Chapter 5 explains the design and fabrication of a six section generalized Chebyshev ceramic waveguide filter. Inter resonator couplings are realized by various through and blind holes. Filter with transmission zero below and above pass band is realized. Methods to realize negative and positive cross couplings in ceramic waveguide triplets are explained. Fabricated generalized Chebyshev filter response is compared with simulation results. A generalized Chebyshev filter with tuning screws having two ways tuning capability is presented.

An integrated ceramic waveguide diplexer design is presented in Chapter 6. The three port monolithic integrated ceramic waveguide diplexer composed of a transmit (TX) and receive $(\mathrm{RX})$ filter and a common port. A six section TX Chebyshev filter at a centre frequency of $2130 \mathrm{MHz}$ and a bandwidth of $60 \mathrm{MHz}$ is designed with metal coated inductive holes. RX filter of diplexer is composed of six sections cross coupled configuration to realize generalized Chebyshev filter response with transmission zeros below and above pass band. The centre frequency of RX filter is selected $1730 \mathrm{MHz}$ 
with a bandwidth of $\pm 30 \mathrm{MHz}$. Methods to achieve broad band coaxial to waveguide transitions are discussed to be used at the common port of the diplexer. HFSS ${ }^{\mathrm{TM}}$ simulated results are given for the integrated ceramic waveguide diplexer.

Chapter 7 presents the design of a corrugated ceramic waveguide low pass filter. Image parameter analysis for a single section of corrugated waveguide low pass filter is explained. Methods to improve the spurious performance of the ceramic waveguide corrugated low pass filter are explained. EM simulation of a band pass filter composed of ceramic corrugated waveguide configuration is presented.

In Chapter 8, practical aspects of the ceramic waveguide filter and diplexer design are discussed. Different tuning methods for ceramic waveguide resonators are explained. Thermal effects on the resonance condition of the ceramic waveguide resonator are investigated. Power handling capability of ceramic waveguide filters is addressed. The spurious performance of ceramic waveguide resonator is discussed and the methods to improve the spurious performance are explored. The conductor losses owing to surface roughness of the resonator are discussed and simulated.

Lastly, Chapter 9 summarises the basic aspects of this thesis along with advantages and shortcomings for both ceramic filter and diplexer design. The shortcomings of current study and possible extension/ improvement of present work are also included. 


\section{MICROWAVE FILTER DESIGN}

\subsection{Introduction}

Microwave filters are the essential part of the modern cellular communication system. They are used to pass only desired signal spectra and reject all other frequency components. Ideal filters exhibit brick wall transfer function with instantaneous transition at cut-off frequency of the filter and constant group delay in the pass band [3]. In practical filter design process, the transfer function of a low pass prototype filter is approximated first and then it is converted to bandpass, bandstop or highpass filter configuration. Based on the synthesis of low pass prototype, the filter is implemented in a suitable physical structure such as printed circuit, microstrip line, waveguide cavity or ceramic material. The design specifications for a filter are the centre frequency, bandwidth, passband insertion loss, return loss and selectivity. The Q-factor of the resonators defines the selectivity and passband insertion loss of the filter. An appropriate transfer function is selected to approximate the response of the filter based on given specifications. The selectivity of the filter increases by increasing the order of the filter at the expense of higher passband insertion loss. Therefore, transmission zeros are added to the filter response so as to increase the selectivity of the filter with less number of reactive components. Transmission zeros can be realized by several ways, such as by introducing cross coupled paths between non-adjacent resonators or by extracted pole techniques $[4,5]$. A microwave filter can be designed using lumped element ladder network synthesis or by using the coupling matrix approach [6]. Physical implementation of more complex filter transfer functions is easier by using coupling matrix method of filter design. Finally, EM simulation tools can be used to get the 
optimised dimensions of the filter. Practical filters allow post production tuning mechanism in order to compensate the manufacturing discrepancies. This Chapter addresses the basic design procedure of a microwave filter. And a review of different physical implementations of the microwave filters used in cellular base station front end is also presented. Insertion loss design method of microwave filter is used to design the integrated ceramic rectangular waveguide filter.

\subsection{Insertion loss filter design method}

Insertion loss method is a well-known and convenient method to design microwave filters. It allows high degree of control over pass band insertion loss, stop band attenuation and phase of the filter under investigation. It gives a systematic approach to design a fully characterized filter response. All types of filter responses i.e. low pass, band pass, high pass or bandstop can be designed by using insertion loss method. A suitable filter response can be chosen depending on the different design parameters e.g. maximum insertion loss, cut-off rate, phase and attenuation in stop band to meet the required system specifications. The order of filter is equal to the number of reactive elements in the filter and stopband attenuation is directly proportional to the order of the filter. The performance of a filter can be readily improved by increasing its order but at the expense of increased pass band insertion loss. The filter response is characterized by the power loss ratio expressed as[7]

$$
P_{L R}=\frac{P_{\text {in }}}{P_{l}}
$$

Where $P_{\text {in }}$ and $P_{l}$ represent input power available and power delivered to the load respectively. If the source and the load are matched then the insertion loss (IL) of a filter can be expressed as

$$
I L(d B)=10 \log _{10}\left(P_{L R}\right)
$$

If an equal-ripple (Chebyshev) response is chosen for an $\mathrm{N}_{\text {th }}$ order low pass filter, the filter pass band will have ripple level of $1+\mathrm{k}_{1}{ }^{2}$ and the power loss ratio in this case can be expressed as

$$
P_{L R}=1+K_{1}^{2} T_{N}^{2}\left(\frac{\omega}{\omega_{c}}\right)
$$


Thus $\mathrm{k}_{1}^{2}$ determines the pass band ripple level. For larger values of $\mathrm{x}, T_{N}(x) \approx \frac{(2 x)^{N}}{2}$. when $\omega \gg \omega_{c}$, the power loss ratio $\left(P_{L R}\right)$ of the filter becomes

$$
P_{L R}=\frac{K^{2}}{4}\left(\frac{2 \omega}{\omega_{c}}\right)^{2 N}
$$

In recent decades, significant advances in the filter design procedures have been published in the literature but microwave and RF filters still follow the design procedure developed by Cohn in the late 1950s [8] [9]. There are three main steps of this microwave filter design procedure: (1) An equivalent circuit prototype is synthesised based on an established mathematical model [10]; (2) The elements of the equivalent filter network are related to the physical dimensions of the actual filter structure and initial dimensions of the structure are obtained [11, 12]; (3) A final optimisation of the physical filter dimensions is performed to achieve required performance [13, 14]. Figure 2.1 shows the basic steps of a microwave filter design by insertion loss method.

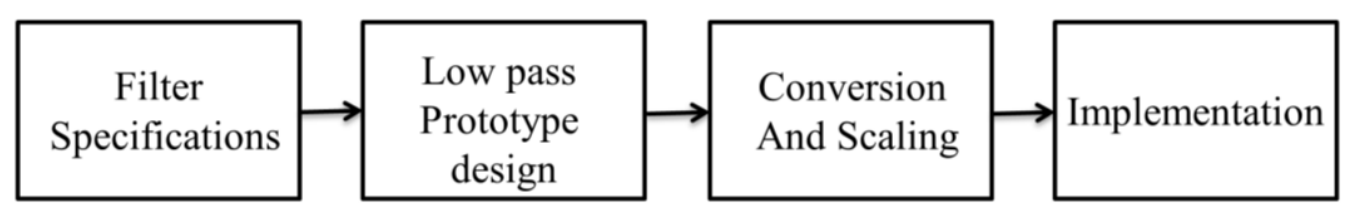

Figure 2.1: Design steps of insertion loss method

An advanced way to achieve reduced sensitivity to manufacturing tolerances, resonator unloaded-Q and filter losses is to use the concept of chained function filters [15-17]. This is achieved by controlling the position of return loss zeros while maintaining a given maximum ripple level in the passband of the filter. Chained function filters provide a number of transfer functions to choose from with each one having different characteristics and different physical implementation requirements. Chained function filters have superior rejection properties as compared to conventional Butterworth filters and slightly less than Chebyshev filters. This family of filters form the basis for tuningless filters while maintaining the minimum return loss level in the passband of the filters [16]. 


\subsubsection{Low pass filter prototype}

Microwave filters can be classified as bandpass, bandstop, lowpass and highpass depending on their characteristics to pass and reject the specified frequency bands. Using circuit synthesis, normalized low pass prototype is the basis for all four configurations. A low pass prototype is a two port passive lumped element reciprocal filter network with $1 \Omega$ source and load impedances and an angular cut-off frequency of $1 \mathrm{rad} / \mathrm{s}$. The order of the filter is determined by total number of reactive elements in the filter. The selectivity of the filter is directly proportional to the order of the filter. The low pass prototype filter can be formed by cascade of a ladder network composed of series inductors and shunt capacitors. The transmitted power in a ladder network is proportional to the product of coupling coefficients for all adjacent resonator pairs [18].

Figure 2.2 shows a generalized low pass prototype filter.

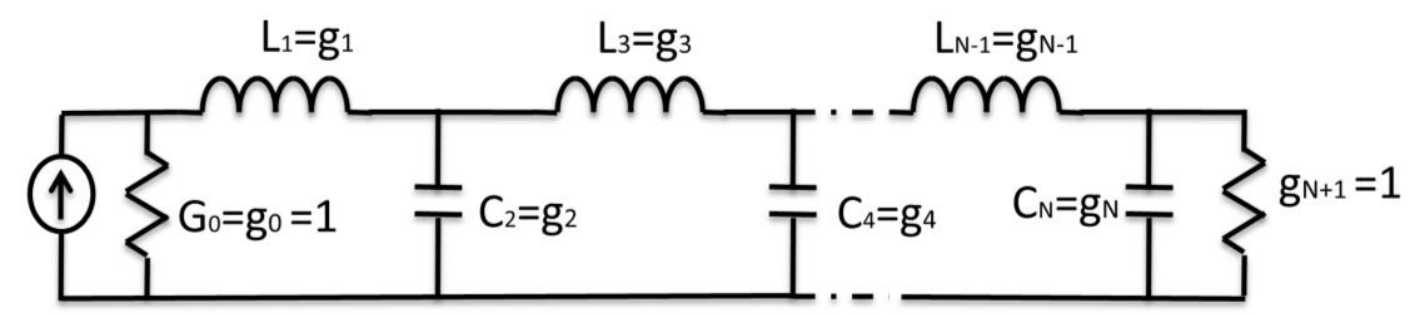

Figure 2.2: Normalized low pass prototype ladder network

An alternative way to realise a ladder network is to use impedance inverters. An impedance inverter as shown in Figure 2.3 is a lossless, reciprocal frequency independent two port network defined by its transfer matrix given as [19]

$$
[T]=\left[\begin{array}{cc}
0 & j K \\
j / K & 0
\end{array}\right]
$$

Where $\mathrm{K}$ is the characteristics admittance or impedance of the inverter and is related to the input and output impedance of the inverter through the relation

$$
K=\sqrt{Z_{\text {in }} Z_{L}}
$$


Thus the input impedance of the inverter is proportional to the inverse of the load impedance.

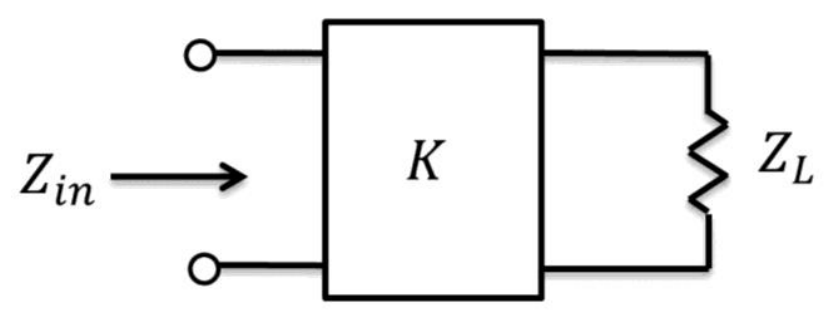

Figure 2.3: Impedance inverter terminated with a $\operatorname{load} \mathrm{Z}_{\mathrm{L}}$

A low pass prototype filter can be designed by using one type of reactive component i.e. either capacitors or inductors and impedance/admittance inverters as shown in Figure 2.4 .

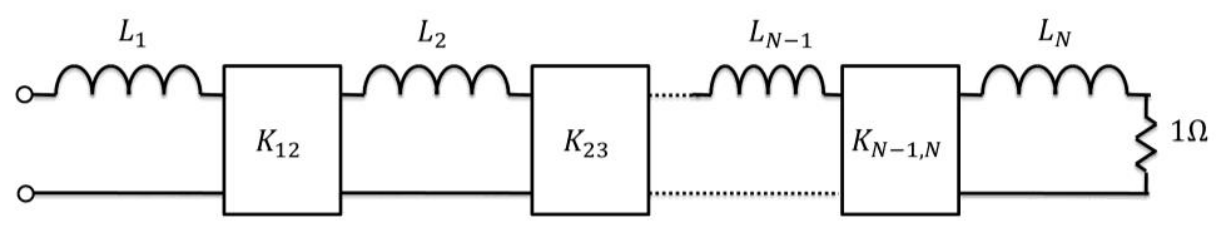

(a)

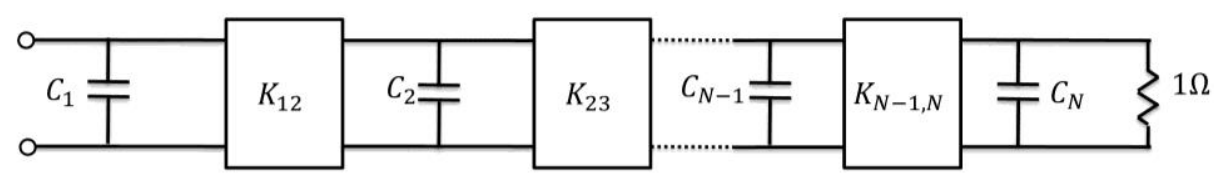

(b)

Figure 2.4: Inverter coupled generalized low pass prototype filter

\subsubsection{Transfer function}

A two port linear time invariant filter network can be represented by its transfer function defined as

$$
\left|S_{21}(\omega)\right|^{2}=\frac{1}{1+\varepsilon^{2} F_{n}^{2}(\omega)}
$$


Where $\varepsilon$ represents the ripple constant, $\omega$ is the frequency variable and $F_{n}(\omega)$ is the characteristic or filtering function. A low pass prototype filter response can be approximated by several transfer functions depending upon the nature of ripple level in the passband and stopband. The insertion loss of the transfer function in Equation 2.7 can be related as

$$
I L(d B)=10 \log \frac{1}{\left|S_{21}(j \omega)\right|^{2}} d B
$$

The insertion loss of a filter is often measured in $\mathrm{dB}$ and represents the attenuation of the signal passing through the filter network.

$$
I L(d B)=20 \log \left|S_{21}(j \omega)\right| d B
$$

Power gain and input reflection coefficient of a two port lossless network cab be related through unitary condition defined as $\left|S_{11}(j \omega)\right|^{2}+\left|S_{21}(j \omega)\right|^{2}=1$, which gives

$$
L_{R}(\omega)=10 \log \left[1-\left|S_{21}(j \omega)\right|^{2}\right] d B
$$

Return loss $\left[L_{R}(\omega)\right]$ of the filter is the measure of attenuation of the reflected signal [3]. It defines the matching level of the filter network. A perfectly matched filter network would have infinite return loss. It is often measured in $\mathrm{dB}$ units and known as return loss of the filter

$$
L_{R}(\omega)=-20 \log \left|S_{11}(j \omega)\right| d B
$$

\subsubsection{Butterworth filter response}

Butterworth approximation is the simplest approximation to an ideal low pass filter prototype. It is maximally flat at $\mathrm{dc}$ and infinity and rolls off to $3 \mathrm{~dB}$ at $\Omega=\frac{\omega}{\omega_{c}}=1$. The Butterworth filter transfer function is given by [3]

$$
\left|S_{21}(j \omega)\right|^{2}=\frac{1}{1+\omega^{2 N}}
$$

Therefore the insertion loss of the filter becomes

$$
I L(d B)=20 \log \left(1+\omega^{2 N}\right) d B
$$

Figure 2.5 shows the response of a maximally flat low pass filter approximation with different values of $\mathrm{N}$, where $\mathrm{N}$ is the order of the filter. The higher is the order; the steeper is the roll off rate of the filter producing sharp transition between passband and stopband. The $3 \mathrm{~dB}$ cut-off frequency of the filter is at $\Omega=1$ where the filter passband 
ends and stopband starts. Butterworth filter offers very flat response in the passband and stopband of the filter. The order of the filter to meet specific attenuation level in the stop band can be calculated as

$$
N \geq \frac{L_{A}(d B)+L_{R}(d B)}{20 \log (S)}
$$

Where

$$
S=\frac{\omega_{s}}{\omega_{p}} \geq 1
$$

$\omega_{s}$ represents stop band frequency and $\omega_{p}$ represents pass band frequency bandwidth of the filter.

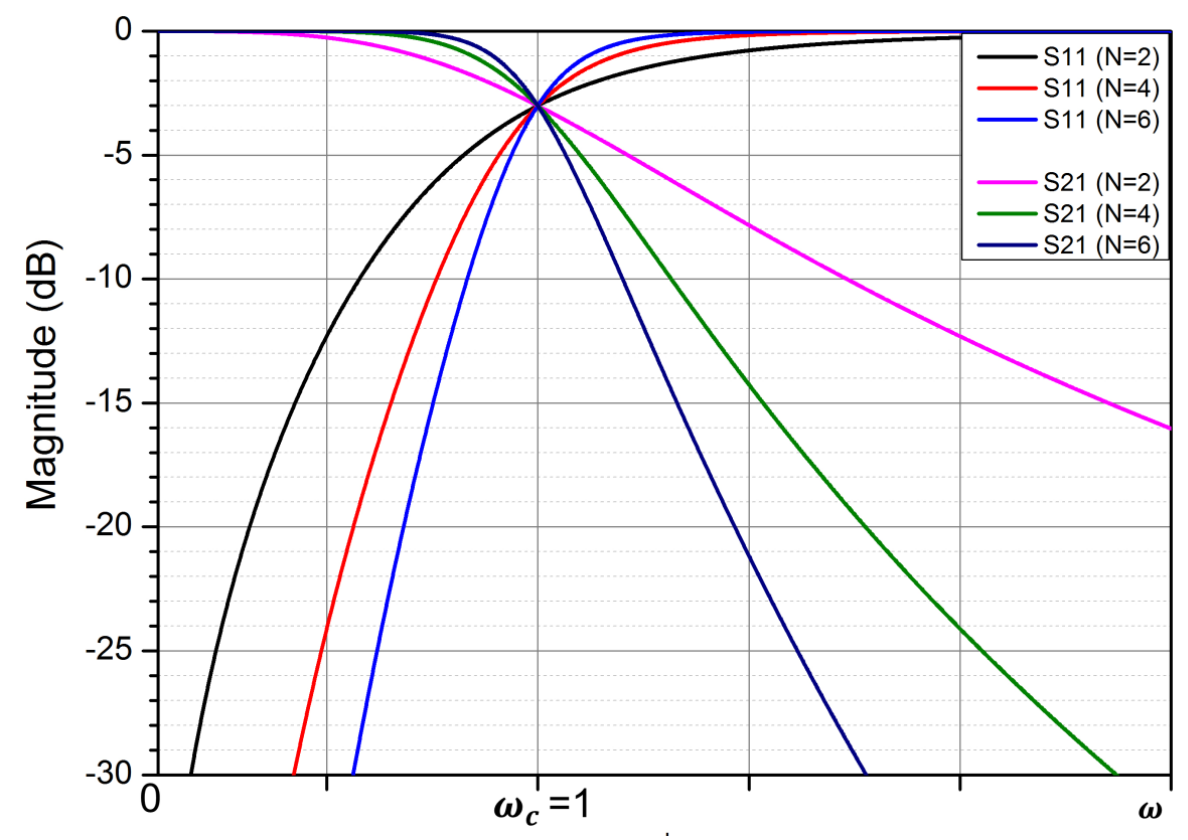

Figure 2.5 : Butterworth low pass filter response

Explicit formulae have been developed for the element values of the general $\mathrm{N}_{\text {th }}$ degree maximally flat inverter coupled lowpass prototype filter as shown in Figure 2.4 and are given below as

$$
\begin{aligned}
g_{r} & =L_{r} \text { or } C_{r} \quad r=1,2 . . N \\
& =2 \sin \left[\frac{(2 r-1) \pi}{2 N}\right]
\end{aligned}
$$


And

$$
K_{r, r+1}=1 \quad r=1,2 . . N-1
$$

\subsubsection{Chebyshev filter response}

Butterworth filter has very flat response in the passband but it does not roll off quickly therefore more number of elements are required to achieve a sharp roll off rate which increases the insertion loss in the passband of the filter. A more suitable approximation is called Chebyshev filter which introduces some ripples between two values in the passband response of the filter up to its cut-off frequency i.e. $\Omega=1$ and then rolls off rapidly in the stopband. The transfer function to achieve Chebyshev filter response is expressed as [20]

$$
\left|S_{21}(j \omega)\right|^{2}=\frac{1}{1+\varepsilon^{2} T_{n}^{2}(\omega)}
$$

Where $\varepsilon$ is the ripple level in the passband and can be evaluated as

$$
\varepsilon=\left(10^{\frac{L_{R}}{10}}-1\right)^{-\frac{1}{2}}
$$

$T_{n}(\omega)$ is the Nth order Chebyshev polynomial which has maximum value of 1 in the passband of the filter. $T_{n}(\omega)$ can be represented mathematically as

$$
T_{n}(\omega)=\operatorname{Cos}\left[N \operatorname{Cos}^{-1}(\omega)\right]
$$

Insertion loss of a Chebyshev filter can be calculated from its transfer function as

$$
I L=10 \log \left[1+\varepsilon^{2} T_{n}^{2}(\omega)\right]
$$

The insertion loss of Chebyshev filter at ripple level becomes

$$
I L=10 \log \left[1+\varepsilon^{2}\right]
$$

The degree of Chebyshev filter to meet any specified rejection level in the stopband can be calculated using the expression

$$
N \geq \frac{L_{A}(d B)+L_{R}(d B)+6}{20 \log \left[S+\left(S^{2}-1\right)^{\frac{1}{2}}\right]}
$$

Where $L_{A}$ is the stopband attenuation, $L_{R}$ is the passband return loss and $S$ represents ratio of stopband to passband frequencies. Figure 2.6 shows the response of a Chebyshev filter with different values of $\mathrm{N}$ when $\varepsilon=0.5$. The formulae to calculate 
element values of a general Nth order Chebyshev filter prototype with impedance inverters as shown in Figure 2.4 are given as [3]

$$
K_{r, r+1}=\frac{\left[\eta^{2}+\operatorname{Sin}^{2}\left(\frac{r \pi}{N}\right)\right]^{\frac{1}{2}}}{\eta} \quad r=1,2 \ldots N-1
$$

And

$$
L_{r}=C_{r}=\frac{2}{\eta} \operatorname{Sin}\left[\frac{(2 r-1) \pi}{2 N}\right] \quad r=1,2 \ldots \ldots N
$$

Where parameter $\eta$ is defined as

$$
\eta=\operatorname{Sinh}\left[\frac{1}{N} \operatorname{Sinh}^{-1}\left(\frac{1}{\varepsilon}\right)\right]
$$

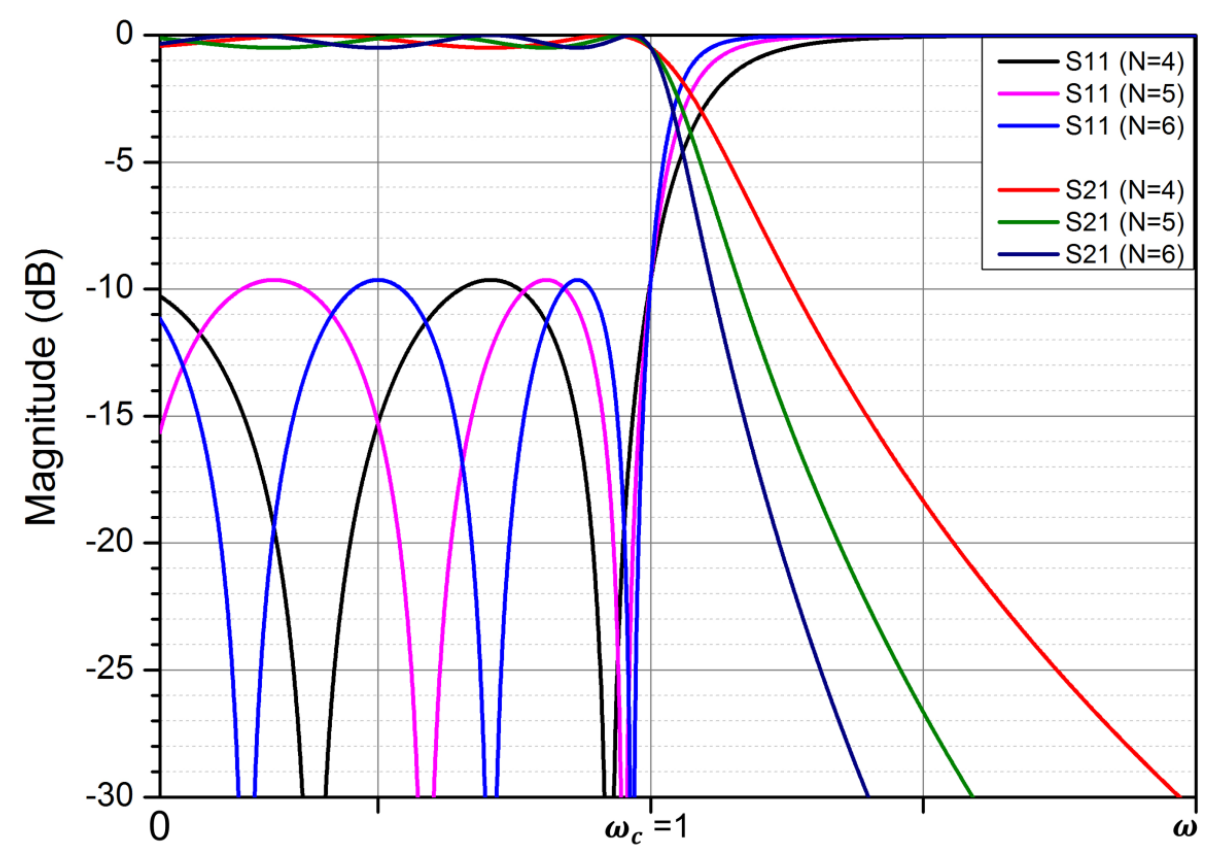

Figure 2.6: Chebyshev low pass filter response

\subsubsection{Generalised Chebyshev filter}

The generalised Chebyshev approximation offers equiripple response in the passband of the filter and arbitrarily placed transmission zeros in the stopband of the filter. As attenuation poles can be placed arbitrarily at any finite frequency. In this way, both symmetric and asymmetric response can be achieved. The generalised Chebyshev 
approximation is given as [3]

$$
\left|S_{21}(\omega)\right|^{2}=\frac{1}{1+\varepsilon^{2} F_{n}^{2}(\omega)}
$$

With

$$
-1<F_{N}(\omega)<+1 \quad \text { for }-1<\omega<+1
$$

And

$$
F_{N}\left(\omega_{r}\right)=\infty \quad r=1,2 \ldots N
$$

Where $\omega_{r}$ is the frequency of rth attenuation pole. Generalised Chebyshev filter provides sharp transition from passband to stopband due to the presence of the transmission zeros. A transmission zero can be produced by several methods such as coupling some amount of energy to the non-adjacent filter elements or by extracted pole techniques [21, 22]. Figure 2.7 shows the response of a generalised Chebyshev filter with a ripple level of 0.5 in the passband and two transmission zeros in the stopband.

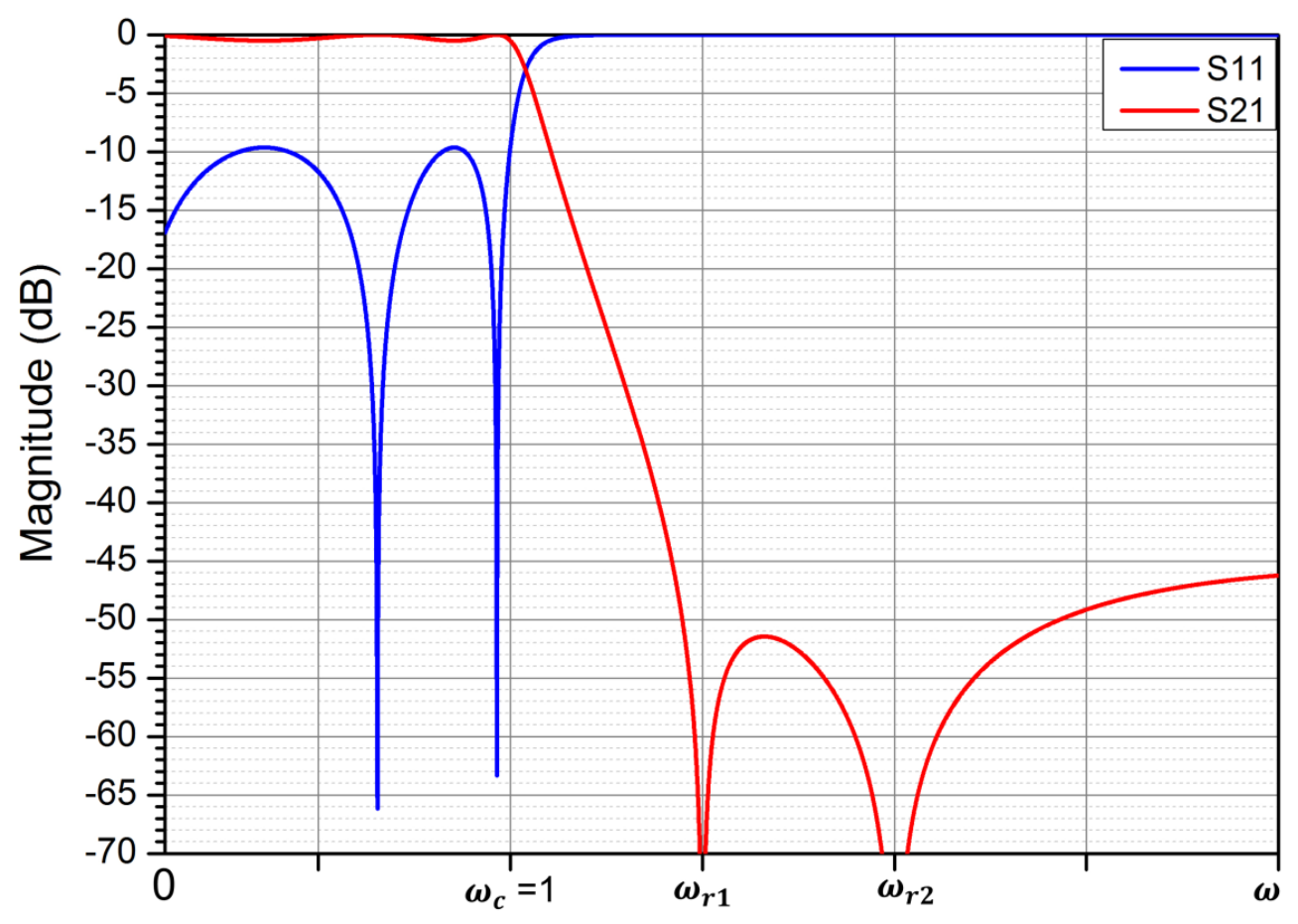

Figure 2.7: Generalised Chebyshev low pass filter response

\subsubsection{Frequency and impedance transformation}

A normalized prototype filter, shown in Figure 2.2, gives a low pass filter response with 
source and load impedance normalized to $1 \mathrm{ohm}$ and the cut-off frequency equals to unity. Most of the communication systems are operated with $50 \mathrm{ohm}$ source and terminations and are operated over a wide range of frequencies. For this, an impedance and frequency scaling of low pass prototype is required. A low pass prototype can be transformed into any low, high or bandpass filter configuration depending on requirement. To scale a low pass prototype to an impedance level of $\mathrm{Z}_{0}$ the scaled filter components can be obtained by following relations [7]

$$
\begin{gathered}
R^{\prime}{ }_{S}=R \\
R_{L}^{\prime}=R \\
L^{\prime}=R . L \\
C^{\prime}=C / R
\end{gathered}
$$

Where primed quantities denote impedance scaled components and $\mathrm{R}, \mathrm{L}$ and $\mathrm{C}$ are the original low pass prototype component values. The cut-off frequency of the low pass prototype can be changed from unity to $\omega_{c}$, where $\Omega$ in the low pass prototype is replaced from unity to $\omega / \omega_{c}$. And the power loss ratio of the transformed filter becomes

$$
P L R^{\prime}(\omega)=P L R\left(\frac{\omega}{\omega_{c}}\right)
$$

Where ' $\omega_{c}{ }^{\prime}$ is the cut-off frequency of the transformed filter. Thus new filter elements with frequency and impedance transformation can be evaluated using the expressions [7]

$$
\begin{aligned}
& L^{\prime}=R \cdot \frac{L}{\omega_{c}} \\
& C^{\prime}=\frac{C}{R \cdot \omega_{c}}
\end{aligned}
$$

\subsubsection{Bandpass transformation}

A low pass prototype can be transformed to give a band pass filter response if $\omega$ is replaced with the following expression [7]

$$
\omega \rightarrow \frac{1}{F_{B W}}\left(\frac{\omega}{\omega_{0}}-\frac{\omega_{0}}{\omega}\right)
$$

where

$$
F_{B W}=\frac{\omega_{2}-\omega_{1}}{\omega_{0}}
$$


And

$$
\omega_{0}=\sqrt{\omega_{1} \omega_{2}}
$$

Where $F_{B W}$ represents the fractional bandwidth and $\omega_{0}$ is the centre frequency of the filter passband. The low pass prototype filter shown in Figure 2.2 is transformed in to a bandpass filter as shown in Figure 2.8.

For a band pass transformation a series inductor in low pass prototype with inductance $L$ is transformed to a low impedance series LC resonator circuit with capacitance and inductance given by Equation 2.39 and 2.40.

$$
\begin{gathered}
L_{S}^{\prime}=\frac{L}{F_{B W} \omega_{0}} \\
C_{S}^{\prime}=\frac{F_{B W}}{L \omega_{0}}
\end{gathered}
$$

Similarly a shunt capacitor of low pass prototype is transformed into a high impedance parallel resonant LC circuit with element values given as in Equations 2.41 and 2.42

$$
\begin{gathered}
L_{P}^{\prime}=\frac{F_{B W}}{C \omega_{0}} \\
C_{P}^{\prime}=\frac{C}{F_{B W} \omega_{0}}
\end{gathered}
$$

The use of this synthesis method gives very satisfactory results for narrow to moderate bandwidth and, therefore, it is applicable to a variety of microwave bandpass filter applications [23].

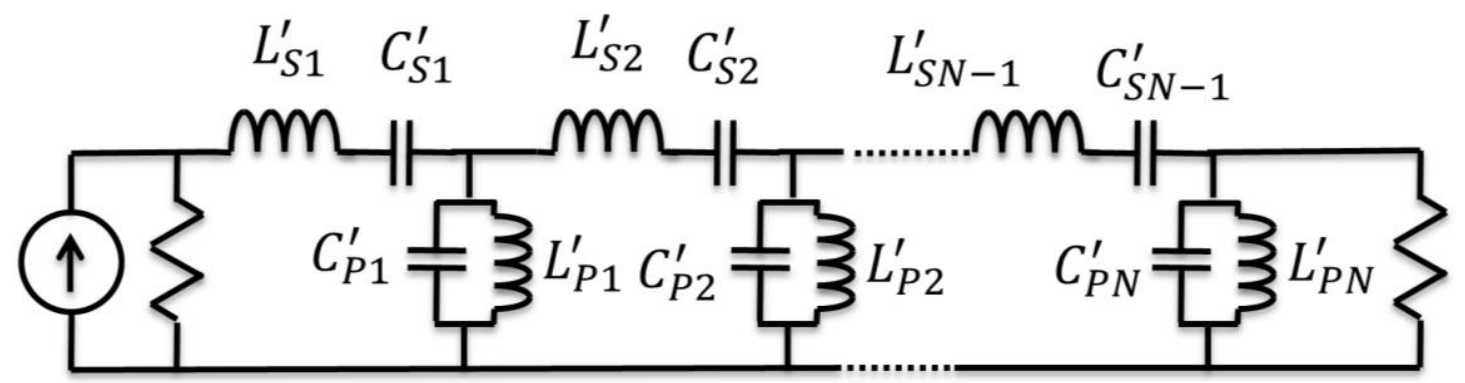

Figure 2.8: Bandpass impedance and frequency scaled filter

\subsection{Implementation of microwave filters}

The final step of a microwave filter design is its implementation in a suitable 
technology. The choice of resonator type is based on the required specification e.g. maximum passband insertion loss, Q-factor, volume, power handling and selectivity of the filter. Conventional cellular base stations use air filled coaxial resonator or waveguide cavity filters. Coaxial resonator filters offer good spurious performance, reliability and simplicity. But they are bulky and possess high inherent loss owing to lower Q-factor values. To enhance their insertion loss performance, dielectric rod is used instead of metal rod which makes the Q-factor of the resonator approximately double as compared to the conventional air filled coaxial comb line filter but have worse spurious performance and require big volumes [24]. On the other hand air-filled empty waveguide filter offers simple structures with very high Q-values ranging from 10,000 to 50,000 , thus, making them suitable to design very low loss devices. Waveguide resonators suffer from the very same problem of bigger size which is the key challenge of miniaturized communication systems. A survey of most often used techniques in microwave filter design is presented by Levy [25]. Dielectric resonator filter was first introduced by Cohn in 1968 using a dielectric material titanium dioxide $\left(\mathrm{TiO}_{2}\right)$ of relative permittivity 100 and loss tangent 0.0001 [26]. Up to 1980's DR filters were not preferred due to their temperature instability. In the late 1980's, advancements in ceramic materials and space and satellite communication focused the attention of researchers towards dielectric resonator filters. A ceramic puck held at the centre of a conducting enclosure resonates at a centre frequency. The resonant frequency of the dielectric structure depends upon shape, diameter and permittivity of the material [3]. Most of the coupled energy is stored within the dielectric medium at its resonant frequency. Mansour compares various filter types used in wireless base stations including TEM coaxial, dielectric resonator filters and HTS (High temperature superconducting) filters [27]. The size, mass, cost, structure, material, power handling capability and temperature drift of various filters is explained. The dielectric resonator filter technology with regard to satellite communication payload, military and radar application and cellular radio base station applications is discussed by Zaki [28]. An explanation of lumped element filters, coaxial resonator, waveguide resonator, ceramic dielectric resonators and superconductor filters is given. A brief introduction of conventional metallic resonators, ceramic loaded resonators and waveguide resonator filters used in cellular base stations is given in 2.3 subsections. 


\subsubsection{TEM coaxial resonator filters}

Air-filled metallic coaxial resonators are commonly used in conventional base station filters. A circular or rectangular metallic rod is placed at the centre of the rectangular cavity. One end of the inner conductor is grounded and the other is open. For an airfilled combline resonator; cavity size, $\mathrm{Q}$ factor and spurious performance are dictated by the inner rod radius to outer cavity radius ratio. These metallic resonators have low production cost, wide spurious performance and wide tuning range but require big volumes and have high inherent loss [29]. Figure 2.9 shows the physical layout and equivalent circuit of the coaxial resonator.

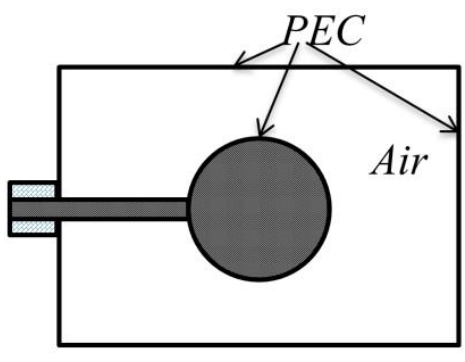

(a)

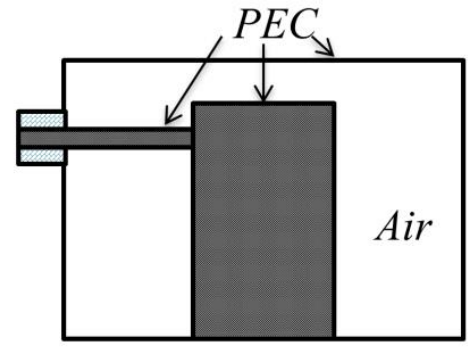

(b)

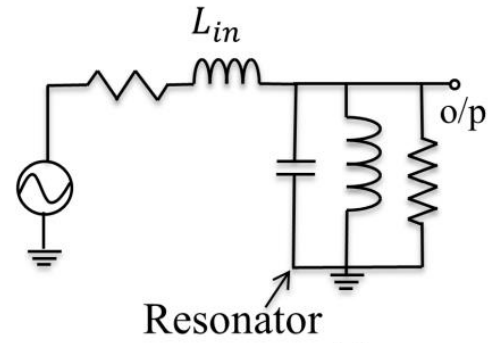

(c)

Figure 2.9: TEM coaxial resonator (a) Top view (b) Side view (c) Equivalent circuit

\subsection{2 $\mathrm{TM}_{01}$ ceramic comb-line filter}

Conventional metallic resonators have high inherent conduction loss due to the presence of inner metallic rod. This loss can be reduced by replacing inner metallic conductor with a high permittivity dielectric rod. Dielectric combline resonators have both the dielectric and combline resonator merits thus they offer high Q (approximately double than air filled comb line filter), wide spurious free window and simple structures [29]. Unfortunately, there is no optimum relationship for outer cavity to inner conductor ratio for maximum Q. Figure 2.10 shows the configuration of a ceramic rod comb-line filter and its equivalent circuit. 


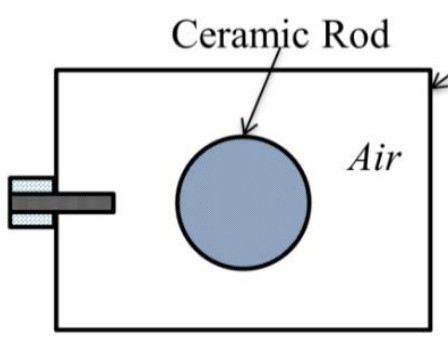

(a)

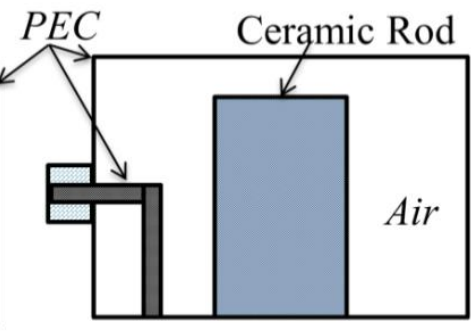

(b)

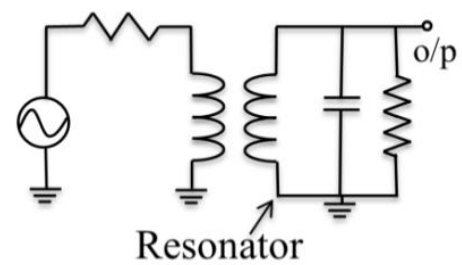

(c)

Figure 2.10: $\mathrm{TM}_{01}$ Ceramic comb-line resonator (a) Top view (b) Side view (c) Equivalent circuit

An example of eight pole elliptical dielectric comb-line filter is designed and explained by Atia [24]. It is found that the fundamental mode is quite sensitive to the variations in the length of rod. If the overall size is kept constant, dielectric combline filter loss is decreased to half as compared to the air-filled coaxial combline filter. Rod diameter, length and proximity determine the overall behaviour of the resonator such as resonant frequency, spurious free bandwidth and Q factor of the resonator [24]. It is suggested to replace conventional metallic comb-line rod by high permittivity dielectric rod in order to get high Q-factor without distorting the spurious response of metallic comb-line filter. The merits of conventional coaxial resonators (low cost, wide tuning range, best spurious performance but low Q) and dielectric resonators (high Q, low temperature stability but higher cost and worse spurious characteristics) are compared. By replacing inner metallic rod with high permittivity dielectric rod, additional losses occurring in conventional metallic combline resonator due to large currents induced on the surface of inner conductor are reduced [24]. Also spurious performance of conventional dielectric resonator is improved. A rigorous mode matching method is used to model dielectric comb-line resonators. Dielectric rod comb-line resonator offers Q-factor twice than the conventional metallic combline resonator. Resonant frequency and unloaded $\mathrm{Q}$ factor of the resonator both are inversely proportional to the dielectric rod length and radius. Dielectric rod with smaller diameter gives better spurious performance. Comprehensive data about rod dimension versus its effects on fundamental and higher order modes is given. 


\subsubsection{Dielectric resonator filters}

The idea of dielectric resonators filters is not new. In the late 1960's, Cohn designed a dielectric resonator filter using dielectric material with permittivity of 100 and loss tangent 0.0001 with Q-factor of 10,000 [26]. Dielectric resonator is formed by placing a ceramic puck at the centre of the conducting box which resonates at the centre frequency due to difference of its permittivity and that of surroundings. At its resonant frequency, most of the electric and magnetic energy is stored within the resonator. The field outside the ceramic puck is decaying and vanishes rapidly at a distance away from the puck. The conductive enclosure stops radiating field outside. Dielectric resonators structures have infinite number of resonant modes, therefore, they are also used to design multimode dielectric resonator filters. The resonant frequency of the dielectric resonator depends upon the shape, diameter and permittivity of the material [3]. Figure 2.11 shows the side view of a ceramic puck in a metal cavity. The ceramic puck does not interact with the conducting enclosure and it is held at the middle of the metal box by using a support of low dielectric material.

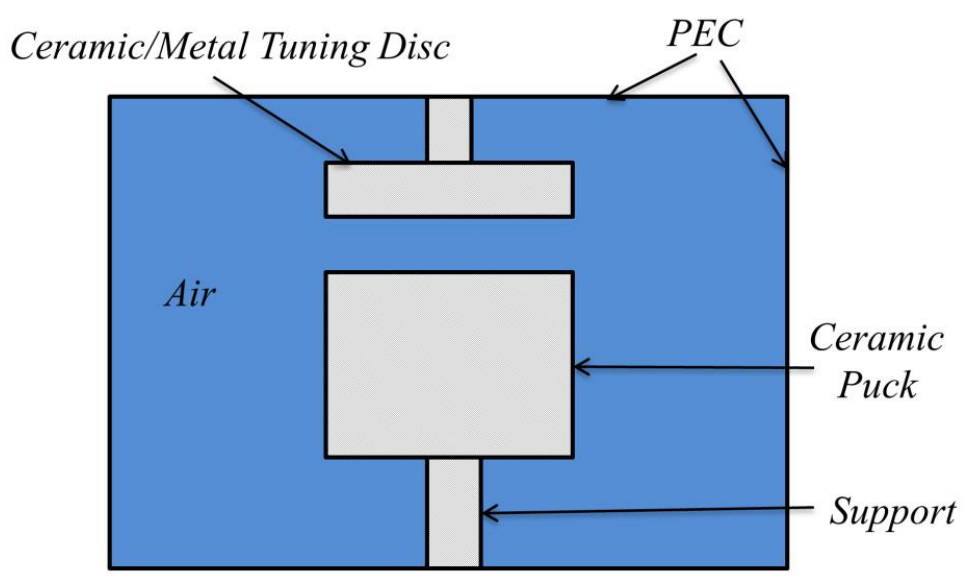

Figure 2.11: $\mathrm{TE}_{01 \delta}$ Dielectric resonator filter (Side view)

Dielectric materials are evaluated based on their relative permittivity, temperature coefficient, and Q value due to dielectric loss [30]. Table 2-1 obtained from [31] summarizes some common dielectric materials used in microwave components. A detailed mathematical explanation of resonant frequencies, filed distribution and magnetic coupling for dielectric resonator band pass filters is discussed by Cohn [26]. 
Author investigates different geometries of dielectric resonators, their properties and derives the formulae for adjacent resonator couplings for a dielectric puck in a metal waveguide operating below its cut-off frequency. Metal enclosure is needed to stop the radiation loss by providing shielding. Also different methods for external coupling such as probe or loop coupling for multi-resonator band pass filter are discussed. And it is concluded that dielectric resonators offer great size reduction as compared to other available resonators with similar Q, but temperature sensitivity of the material still remains a drawback for them [26].

Table 2-1: Commonly used ceramic material at microwave frequencies [31]

\begin{tabular}{|c|c|c|c|c|}
\hline Material & Permittivity & $\mathbf{Q}$ & $\begin{array}{c}\text { Frequency } \\
(\mathbf{G H z})\end{array}$ & $\begin{array}{c}\tau_{f} \\
\mathrm{ppm} /{ }^{0} \mathrm{C}\end{array}$ \\
\hline $\mathrm{MgTiO}_{3}-\mathrm{CaTiO}_{3}$ & 21 & 8000 & 7 & 0 \\
\hline $\mathrm{Ba}(\mathrm{Mg}, \mathrm{Ta}) \mathrm{O}_{3}$ & 25 & 16000 & 10 & 3 \\
\hline $\mathrm{Ba}(\mathrm{Mg}, \mathrm{Ta}) \mathrm{O}_{3}$ & 25 & 35000 & 10 & 4 \\
\hline $\mathrm{Ba}(\mathrm{Sn}, \mathrm{Mg}, \mathrm{Ta}) \mathrm{O}_{3}$ & 25 & 20000 & 10 & 0 \\
\hline $\mathrm{Ba}(\mathrm{Mg}, \mathrm{Ta}) \mathrm{O}_{3}-\mathrm{Ba}(\mathrm{Zn}, \mathrm{Ta}) \mathrm{O}_{3}$ & 27 & 15000 & 10 & 0 \\
\hline $\mathrm{Ba}(\mathrm{Zn}, \mathrm{Nb}) \mathrm{O}_{3}-\mathrm{Ba}(\mathrm{Zn}, \mathrm{Ta}) \mathrm{O}_{3}$ & 30 & 14000 & 12 & 0 \\
\hline $\mathrm{Ba}(\mathrm{Zr}, \mathrm{Zn}, \mathrm{Ta}) \mathrm{O}_{3}$ & 30 & 10000 & 10 & 0 \\
\hline$(\mathrm{Ca}, \mathrm{Sr}, \mathrm{Ba}) \mathrm{ZrO}_{3}$ & 30 & 4000 & 11 & 5 \\
\hline $\mathrm{BaO}-\mathrm{TiO}_{2}-\mathrm{WO}_{3}$ & 37 & 8800 & 6 & 2 \\
\hline$(\mathrm{Zr}, \mathrm{Sn}) \mathrm{TiO}_{4}$ & 38 & 7000 & 7 & 0 \\
\hline $\mathrm{Ba}_{2} \mathrm{Ti}_{9} \mathrm{O}_{20}$ & 40 & 8000 & 4 & 2 \\
\hline $\mathrm{Sr}(\mathrm{Zn}, \mathrm{Nb}) \mathrm{O}_{3}-\mathrm{SrTiO}_{3}$ & 43 & 5000 & 5 & \pm 5 \\
\hline $\mathrm{BaO}-\mathrm{Sm}_{2} \mathrm{O}_{3}-5 \mathrm{TiO}_{2}$ & 77 & 4000 & 2 & 15 \\
\hline $\mathrm{BaO}-\mathrm{PbO}-\mathrm{Nd}_{2} \mathrm{O}_{3}-\mathrm{TiO}_{2}$ & 90 & 5000 & 1 & 0 \\
\hline
\end{tabular}

A comprehensive discussion about dielectric resonator, its properties, resonant modes, different analysis models, coupling of resonators, their applications and comparison of dual verses single mode performance is explained by Zaki [32]. Author discusses about single mode, dual mode and mixed mode dielectric resonators and concludes that single 
mode DR filters are simple to design and flexible while dual mode filters provide substantial advantage in mass and volume reduction. A combination of both single mode $\mathrm{TE}_{01 \delta}$ and dual mode $\mathrm{HE}_{11} \mathrm{DR}$ filter offer both higher $\mathrm{Q} /$ volume ratio and good spurious performance. A single mode DR, transmit and receive filter with nine resonant sections for the UMTS application is presented by Knack [33]. A TM mode eight pole Chebyshev DR filter with three transmission zeros suitable for $3 \mathrm{G}$ cellular mobile communication and LTE is investigated in [34]. A 30\% volume reduction as compared to conventional coaxial resonator filter is achieved. The proposed configuration has higher Q from both metallic combline filter and both end metalized TM mode dielectric resonator. Pance introduced a moving DR structure coupling technique to design multiband tuneable dielectric resonator filter [35]. A band pass ceramic filter is designed in [36]. A high Q state of the art single mode dielectric resonator filter for PCS communication base station filters is designed in [37]. The summary of technological innovation of single mode dielectric resonator filters for wireless communication base station systems is given. A detailed explanation of $\mathrm{TE}_{01}$ mode ceramic filter and half wave $\mathrm{TM}_{01}$ mode ceramic filter is given in this paper. It is investigated that $\mathrm{TE}_{01}$ mode filter with planar layout offers significant advantage over in line filter configuration. Development of cross coupling techniques has enhanced performance of $\mathrm{TE}_{01}$ dielectric resonator filter similar to dual mode dielectric resonator filter. Also $\mathrm{TE}_{01}$ mode dielectric filter has virtues of simple design, flexible layout and cheaper manufacturing with respect to $\mathrm{HE}_{11}$ dual mode filters. However, they are bigger in volume and heavy in weight. A combination of dielectric resonator filter integrated with combline filter is presented in [38]. This integrated structure merges the virtues of dielectric resonator filter and conventional combline filter, thus offering high $\mathrm{Q}$, minimal complexity, cost effectiveness and excellent spurious performance. A four pole dielectric resonator filter integrated with six pole metallic combline filter operating at WiMax band in $\mathrm{TE}_{01}$ configuration is designed and tested. Tuning procedure and practical design considerations of the proposed design are also explained. Sophisticated coupling structure is introduced to couple energy to and from dielectric resonator filter and combline filter. A 10 pole dielectric resonator filter without irises suitable for base stations is investigated in [39]. The overall filter structure consists of movable canonical shape dielectric resonators mounted alternatively in orientation. Their movable capability provide full control over inter resonator coupling and hence avoids the need 
of coupling irises and makes it easy to tune coupling bandwidths and resonances. To boost spurious performance two comb-line cavities are used as first and last resonator in conjunction with dielectric resonator cavities. A new class of dual mode dielectric resonator filter i.e. conductor loaded DR filter for GSM transmit band is demonstrated in [40]. The geometry, coupling, field pattern and resonant modes of the conductor loaded dielectric resonator filter are explained. The device has less than half the size of TEM filters with the same Q. Dual degenerate mode $\mathrm{HE}_{11}$ is the fundamental mode of the structure composing of a dielectric puck resting on the base of metallic cavity and a conducting metallic disc on the top of it. The conducting disc drives the fundamental mode down in frequency and achieves reduction in size on the lower frequency. Permittivity of resonator, diameter of puck and disk determine the resonant frequency of the structure. Height of the puck has no significant effect on frequency, but contributes to the unloaded $\mathrm{Q}$ of the resonator. The internal couplings are realized by slots in the disc at an angle of 45 to the modal vectors. Slot depth determines the coupling bandwidth. Shorted coaxial stubs are placed in the irises to achieve inter resonator couplings. Conductor loaded dielectric resonator filter provides twice the $\mathrm{Q}$ with the same volume as compared to conventional combline filters. A miniaturized low loss fourth order elliptical dielectric loaded combline filter is implemented in [41]. It allows $75 \%$ volume reduction as compared to air-filled coaxial resonator filter by using 80 degree dielectric loaded coaxial resonators. It exhibits very low insertion loss in the pass band of the filter.

\subsubsection{Air-filled waveguide filter}

A half wavelength waveguide section shorted from both sides forms a cavity resonator. Electric and magnetic fields are stored within the cavity and the power can be dissipated in the conducting walls and the dielectric filling. Figure 2.12 shows the layout of a rectangular waveguide cavity filter. $\mathrm{TE}_{10}$ is the fundamental mode of a rectangular waveguide. Coupling from one resonator to another can be achieved through capacitive or inductive windows in the resonator common wall. External couplings can be achieved by placing a coaxial probe at the centre of the broad wall. Waveguide filters are usually reasonable where very high Q-factor is required. 


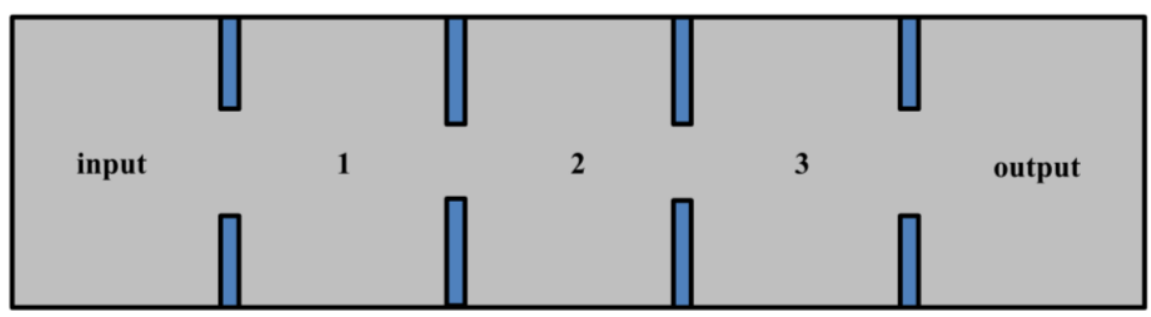

(i)

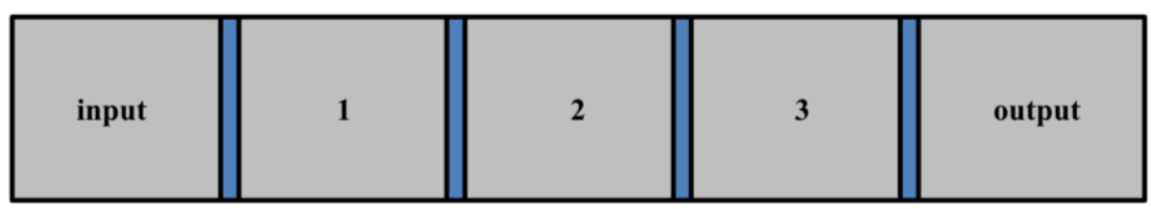

(ii)

Figure 2.12: Air filled rectangular waveguide cavity filter (i) Top view (ii) Side view

A C-band (4-8 GHz) direct coupled cavity waveguide filter is designed using MathCAD mathematical software in [42]. The designed structure is based on insertion loss designing method and physical realization is achieved using rectangular waveguide cavities, which is appropriate for high power applications. Circular inductive posts are implemented in between half wave resonators to provide inter-resonator couplings. These posts behave as inductive inverters on broad bandwidths. An interactive mathematical software tool to design direct coupled cavity filters is developed. A $7^{\text {th }}$ order $\mathrm{Ku}$-Band iris coupled waveguide filter realization is reported in [43]. The structure is realized in rectangular waveguide configuration operating in its $\mathrm{TE}_{10}$ resonant mode. High power handling capability and high Q factor are the virtues of the design. The synthesis procedure of the filter, coupling arrangements and coaxial to waveguide transition are discussed in detail. The specific design formulae for waveguide filter design are given in [8]. The design and implementation of a rectangular waveguide filter using simulation tools based on Wave iterative method is presented in [44].

\subsubsection{Dielectric loaded waveguide filter}

Waveguide resonators can be made with a cavity of half of the guide wavelength long and short circuited on both sides. The size of the air filled waveguide cavity can be reduced by loading it with the ceramic material. The $\mathrm{Q}$ factor and physical dimensions 
of the dielectric waveguide resonator decreases by a factor of $1 / \sqrt{\varepsilon_{r}}$ as compared to empty waveguide. Generally quality factor $\mathrm{x}$ frequency (QF) product of dielectric materials decreases as dielectric constant is increased [45]. A dielectric loaded rectangular waveguide design and analysis is reported in [46]. $\mathrm{TE}_{10}$ mode excited design offers miniaturization with wideband spur free bandwidth and no tuning is required.

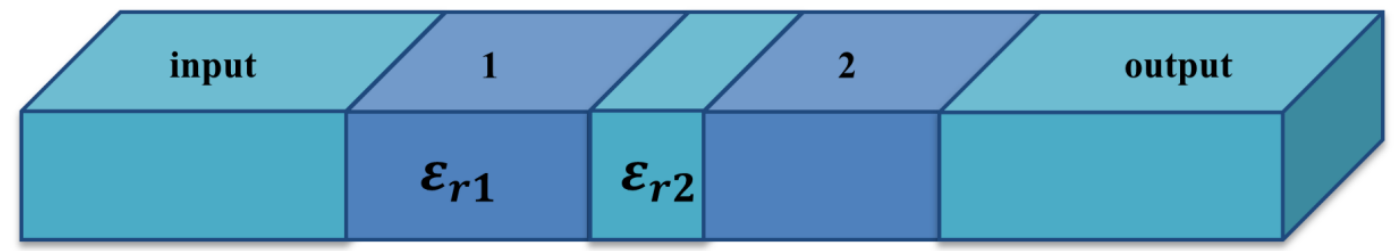

Figure 2.13: Dielectric loaded waveguide filter

Practical issues in designing the filter are discussed and it is shown that this structure has superior spur response over inductive window coupled resonator filters. One of the presented configuration is implementable with elliptical and Chebyshev response. Author discusses full wave analysis and design procedure in detail. Fiedziuszko describes dielectric waveguides and components, microwave dielectric material properties, currently available dielectric materials and their power handling characteristics in detail [47]. In the former section, the authors explore dielectric resonator structures and filters. Konishi describes the concept of creating new dielectric waveguide components, dielectric waveguide to microstrip transition and dielectric waveguide resonators. Multistage band pass filter response is achieved using tandem connection of quarter wave waveguide resonators. Several dielectric filter design considerations were compared against hollow metallic waveguides. Coupling to waveguides is achieved by probes or via open surfaces representing the magnetic walls between resonators [31]. A compact dielectric filled waveguide band pass filter operating at X-band (8-12 GHz) is reported by Ghorbaninejad [48]. Dielectric filled sections are separated by $\mathrm{N}+1$ inductive diaphragms in a rectangular waveguide where $\mathrm{N}$ represents total number of resonators. It is claimed that proposed structure achieves $57 \%$ miniaturization as compared to conventional air filled waveguide filters. The proposed structure is easy to manufacture, have capability to handle high powers and mechanically stable. Detailed mathematical explanation about coupling windows and 
impedance inverters is added in the paper. Hayashi presents the design of a low temperature co-fired ceramic (LTCC) waveguide filter operating in EHF (30-300 GHz) band [49]. Finite element method is used to analyse the circuit simulation. Author gives detailed formula of equivalent circuit model for coupling coefficients, resonator lengths and susceptances to be used. Two monolithic integrated dielectric-filled metal-pipe rectangular waveguide structures are presented by Lucyszyn [50]. Simulated and measured performance of a metal-pipe dielectric-filled rectangular $\pi$-network is presented, to be used at millimetre wave frequency spectrum. Only $\mathrm{TE}_{10}$ mode of rectangular waveguide is considered as the height of waveguide is very small as compared to its width. The high insertion loss is attributed to the available lossy material, non-ideal coplanar waveguide-to-waveguide transition and measurement facilities .The fabrication of a dielectric-filled rectangular waveguide using an easy and low cost photo-image-able thick film processing is described by Lucyszyn [51]. The size of the waveguide operating in the E-band $(60-90 \mathrm{GHz})$ is comparable to the planar structures.

\subsubsection{Integrated ceramic waveguide filter}

In this thesis, the design and implementation of monolithic integrated ceramic waveguide filters suitable for cellular base station is presented. A half wavelength long metal coated ceramic rectangular block behaves as a waveguide cavity. Physical dimensions of the ceramic rectangular waveguide resonator, operating in $\mathrm{TE}_{10}$ fundamental rectangular waveguide mode are reduced by a factor of $\frac{1}{\sqrt{\varepsilon_{r}}}$, as compared to air-filled waveguide cavity. Therefore significant reduction in overall volume of the filter can be achieved by using high permittivity ceramics. The ceramic rectangular waveguide resonator properties are discussed in detail in Chapter 3 and the design of a Chebyshev and a generalized Chebyshev integrated ceramic waveguide bandpass filters is presented in Chapter 4 and Chapter 5, respectively.

\subsection{Summary}

A microwave bandpass filter can be designed using insertion loss method of filter design. A normalized low pass prototype filter is synthesized first by using ladder network. The lowpass prototype is transformed into a bandpass configuration and then it 
is impedance scaled. The lumped element bandpass filter is then implemented in a suitable physical resonator technology depending upon application, insertion loss, mass and volume requirement. A review of commonly used microwave filter technologies is presented in this Chapter. 


\section{CERAMIC WAVEGUIDE}

\section{RESONATOR}

A waveguide is a hollow/dielectric filled structure without a centre conductor with finite lower cut off frequency. If a waveguide cavity is cut at half guide wavelength section and shorted from both sides, it behaves as a resonator. Air-filled waveguide resonators have very high Q-factor up to 50,000 and relatively high power handling capability. However, they suffer from drawback of bigger volumes at lower part of microwave spectrum. The most common shapes for waveguides are rectangular waveguides and cylindrical waveguides. Usually, at microwave frequencies a rectangular waveguide with an aspect ratio of $\mathrm{b} / \mathrm{a}=0.5$ is mostly used [52].

Ceramic waveguide resonators can be made by truncating a piece of rectangular ceramic material at half of the guide wavelength and assigning conducting boundary on all of its walls. Electric and magnetic energy is stored within the structure and produce resonance condition. The $\mathrm{Q}$ factor and the physical dimensions of the dielectric waveguide resonator decrease by a factor of $\frac{1}{\sqrt{\varepsilon_{r}}}$ as compared to hollow waveguide. Generally, QF product of dielectric materials decreases as dielectric constant is increased [45]. Figure 3.1 shows a ceramic rectangular waveguide resonator. The fundamental TE mode resonant frequency of a half wavelength ceramic waveguide resonator can be calculated as [20],

$$
f_{0}=\left(\frac{1}{2 \sqrt{\varepsilon_{0} \varepsilon_{r} \mu_{r}}}\right) \sqrt{\left[\left(\frac{l}{a}\right)^{2}+\left(\frac{m}{b}\right)^{2}+\left(\frac{n}{d}\right)^{2}\right]}
$$


Where $l, m$ and $n$ represent half wavelength variation of electric field lines along the width (a), height (b) and the length (d) of the resonator, respectively. For $\mathrm{TE}_{10}$ fundamental mode of the ceramic waveguide resonator, it is evident from Equation 3.1 that the resonant frequency only depends on width and length of the resonator and it is regardless of the waveguide narrow wall. Thus, waveguide resonator narrow wall does not contribute to the resonant frequency of the resonator but higher order modes and Qfactor of the resonator depend on the height of the resonator. If the height of the resonator is increased, the overall Q-factor tends to increase but at the same time higher order modes get closer to the fundamental resonant mode and makes its spurious performance worse. Therefore, rectangular waveguide resonator height is often kept half of its broad dimension, which is a trade-off between Q-factor and spurious performance of the resonator.

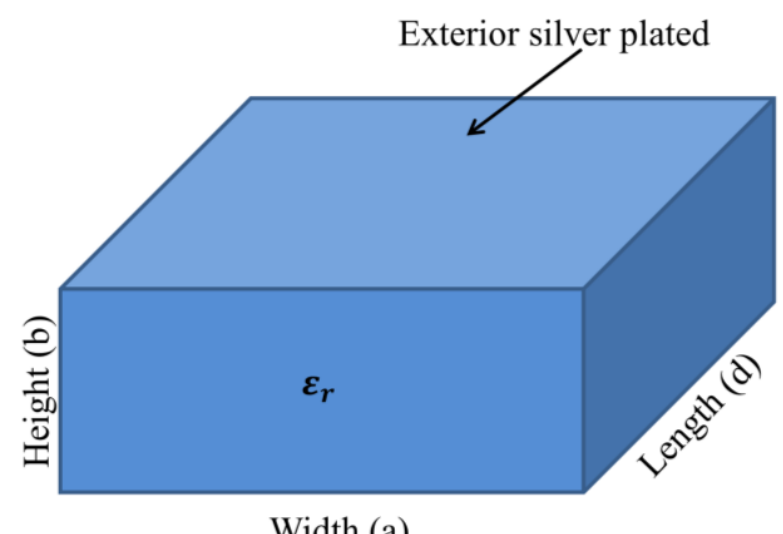

Figure 3.1: Ceramic waveguide resonator

\subsection{Rectangular waveguide modes}

A waveguide can support infinite number of modes either TE or TM not having their electric or magnetic field component in the direction of propagation respectively. Transverse electromagnetic (TEM) mode does not exist in waveguides because waveguides are single conductor transmission media [7]. For a rectangular waveguide, the fundamental dominant mode of propagation is $\mathrm{TE}_{10}$ [3]. Where TE refers to transverse electric and ' 10 ' represents half wave variations of electric field along the width and height of the waveguide, respectively. The field solutions for fundamental $\mathrm{TE}_{10}$ mode in rectangular waveguide with $\mathrm{a}>\mathrm{b}$ are given as, [52] 


$$
\begin{gathered}
E_{y}=-\frac{j a \omega \mu}{\pi} \mathrm{H}_{0} \sin \left(\frac{\pi x}{a}\right) \\
H_{x}=\frac{j a \beta}{\pi} \mathrm{H}_{0} \sin \left(\frac{\pi x}{a}\right) \\
H_{z}=H_{0} \cos \left(\frac{\pi x}{a}\right) \\
E_{x}=E_{z}=H_{y}=0
\end{gathered}
$$

Figure 3.2 shows the E-field and H-field distribution of rectangular ceramic waveguide resonator cavity.

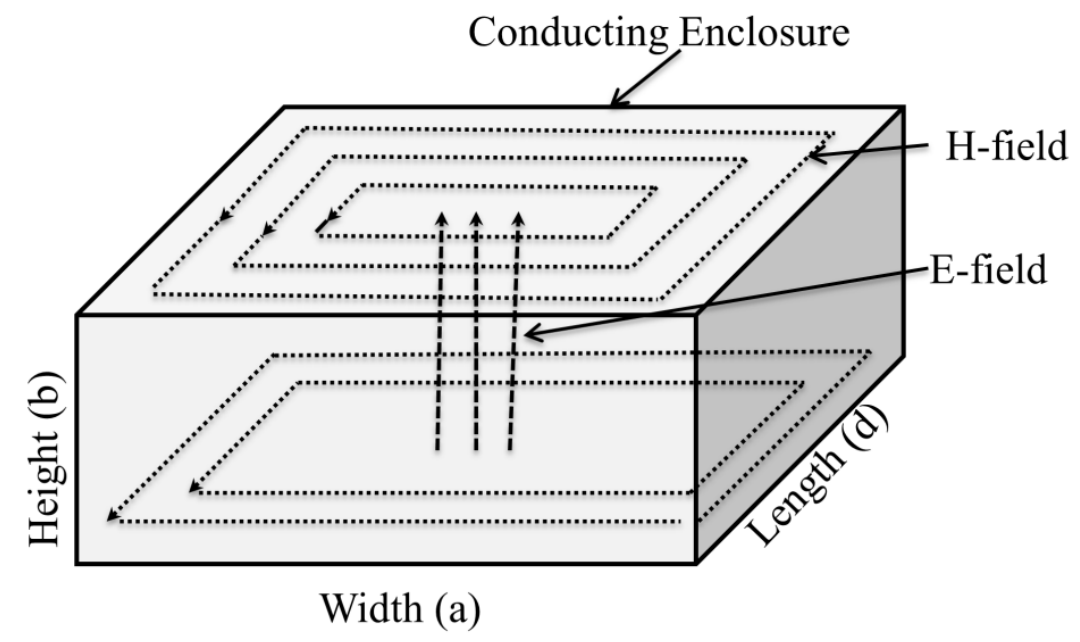

Figure 3.2: Electric and magnetic field pattern of rectangular waveguide resonator

Usually, waveguides are operated in a single mode behaving as transmission lines with an exponentially decreasing propagation constant $\gamma$. In a rectangular waveguide the first higher order mode is $\mathrm{TE}_{20}$, with cut-off frequency double the cut-off frequency of fundamental $\mathrm{TE}_{10}$ mode. Figure 3.3 shows a mode chart of cut-off frequencies of few lowest order modes of a rectangular waveguide with a ratio $b / a=0.5$. Usually cut-off frequency is about $30 \%$ lower than the operating frequency of the waveguide in order to minimise the dispersion caused by group delay due to different frequency components of the wave [53]. The cut-off frequency of the fundamental mode of a rectangular waveguide can be calculated as [7]

$$
\omega_{c}=v\left[\left(\frac{m \pi}{a}\right)^{2}+\left(\frac{n \pi}{b}\right)^{2}\right]^{\frac{1}{2}}
$$


Where ' $v$ ' represents velocity of light in a dielectric medium and for a rectangular waveguide with aspect ratio of $b=a / 2, \omega_{c}$ can be simplified as

$$
\omega_{c}=\frac{v \pi}{a}\left[m^{2}+4 n^{2}\right]^{\frac{1}{2}}
$$

The characteristic impedance of the fundamental mode of the rectangular waveguide can be represented as the ratio of transverse $\mathrm{E}$ and $\mathrm{H}$ fields [3], i.e.

$$
Z_{T E}=\frac{E_{y}}{H_{x}}=\frac{\omega \mu}{\beta}
$$

And the propagation constant is evaluated as

$$
\beta=\left[\omega^{2} \mu \varepsilon-\left(\frac{\pi}{a}\right)^{2}\right]^{\frac{1}{2}}
$$

Since $\frac{\pi}{a}=\frac{\omega_{c}}{v}$, therefore

$$
\beta=\frac{\omega}{v}\left[1-\left(\frac{\omega}{\omega_{c}}\right)^{2}\right]^{\frac{1}{2}}
$$

The propagation constant of the wave propagating in the waveguide is related to the guide wavelength $\left(\lambda_{g}\right)$ by

$$
\beta=\frac{2 \pi}{\lambda_{g}}
$$

Therefore

$$
\lambda_{g}=\frac{2 \pi}{\beta}=\frac{\lambda_{0}}{\left[1-\left(\frac{\omega_{c}}{\omega}\right)^{2}\right]^{\frac{1}{2}}}
$$

And the guide velocity $\left(v_{g}\right)$ becomes

$$
v_{g}=\frac{d \omega}{d \beta}=v\left[1-\left(\frac{\omega_{c}}{\omega}\right)^{2}\right]^{\frac{1}{2}}
$$

As ' $v_{g}$ ' approaches zero, ' $\omega$ ' approaches ' $\omega$ ', ' $\beta$ ' tends to zero and ' $\lambda_{g}$ ' tends to infinity leading to phase distortion as the signal frequency approaches too close to the cut-off frequency of the waveguide. The Figure 3.3 represents the mode chart of a rectangular waveguide resonator with an aspect ratio of $\mathrm{b} / \mathrm{a}=0.5$ in which $f^{2} a^{2}$ is 
plotted as a function of $a^{2} / d^{2}$ for all the TE and TM modes having $1, \mathrm{~m}, \mathrm{n} \leq 2$.

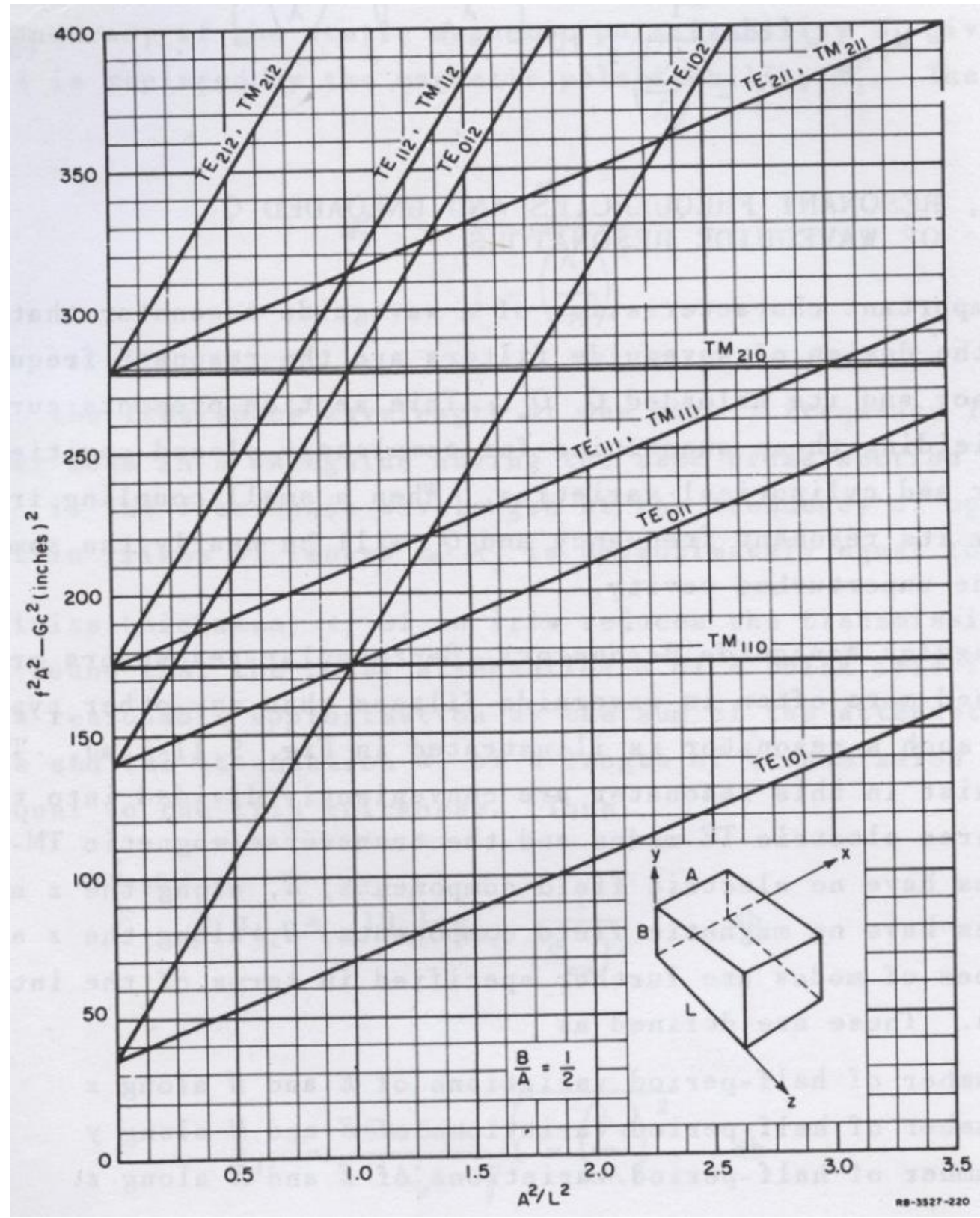

Figure 3.3: The mode chart for rectangular waveguide resonator with $\mathrm{B} / \mathrm{A}=0.5$ [20]

For $\mathrm{TE}_{10}$ mode of rectangular waveguide, propagation occurs along $\mathrm{z}$ direction therefore E-field must be zero at $\mathrm{z}=0$ and $\mathrm{z}=\mathrm{d}$. To form a rectangular cavity resonator length $(\mathrm{d})$ of the resonator must be half guide wavelength long. Hence [3], 


$$
\begin{aligned}
d & =\frac{\lambda_{g}}{2}=\frac{\lambda_{0}}{2\left[1-\left(\frac{\omega_{c}}{\omega}\right)^{2}\right]^{\frac{1}{2}}} \\
& =\frac{\lambda_{0}}{2\left[1-\left(\frac{\lambda_{0}}{2 a}\right)^{2}\right]^{\frac{1}{2}}}
\end{aligned}
$$

And the resonant frequency of the waveguide resonator can be approximated as

$$
f_{0}=\frac{c}{\lambda}=\frac{c\left(a^{2}+d^{2}\right)^{\frac{1}{2}}}{2 a d}
$$

\subsection{Permittivity of resonator}

Permitivity of a dielectric material determines its capability to store electric and magnetic energy at its resoanant frequency when applied with an external potential across it. The speed of the electromagnetic wave travelling through a dielectric material is affected by the permitivity of the material. The higher the permitivity of a material the lower is the speed of microwave signal passing through it [54]. By increasing permitivity of the dielctric resonator material, resonant frequency is decreased but it affects the bandwidth of the dielectric resonator filter. The wavelength passing through a dielectric medium is related with the permitivity of the material as [45]

$$
\lambda_{d}=\frac{\lambda_{0}}{\sqrt{\varepsilon_{r}}}
$$

where $\lambda_{\mathrm{d}}$ represents dielectric wavelength, $\lambda_{0}$ denotes free space wavelength and $\varepsilon_{r}$ is the relative permitivity of the dielectric medium.

\subsection{Q factor}

Quality factor is an important measurement of power loss of a microwave system. It determines the pass band insertion loss, bandwidth and selectivity of a microwave filter. It is inversely related to the bandwidth of the resonator and insertion loss. It is defined as [55] 


$$
Q=2 \pi * \frac{\text { Energy }_{\max } \text { stored per cycle }}{\text { Energy } y_{\text {avg }} \text { dissipated per cycle }}
$$

There are four types of losses that occur in the dielectric resonators, i.e. dielectric loss due to dielectric relaxation, conduction loss due to electric conduction, radiation loss and external loss. Generally, external loss occurs because of external coupling. When coupling energy to the dielectric resonator section, it should be considered that the higher the dielectric constant of the resonator the closer is the coupling to the resonator required. There are three types of the $\mathrm{Q}$ factor defined as loaded, unloaded and the total $\mathrm{Q}$ factor. The unloaded $\mathrm{Q}$ factor accounts for the internal losses of the resonatror, loaded Q factor includes external losses occurred in the dielectric resonator. And the total Qfactor accomodates both loaded and unloaded Q-factor. The unloaded Q factor can be calculated as [55]

$$
\frac{1}{\mathrm{Q}_{\mathrm{u}}}=\frac{1}{\mathrm{Q}_{\mathrm{d}}}+\frac{1}{\mathrm{Q}_{\mathrm{c}}}+\frac{1}{\mathrm{Q}_{\mathrm{r}}}
$$

Where $\mathrm{Q}_{\mathrm{d}}, \mathrm{Q}_{\mathrm{c}}$ and $\mathrm{Q}_{\mathrm{r}}$ represent dielectric , conduction and radiation losses respectively. And

$$
\begin{aligned}
& Q_{d}=\frac{\omega_{0} W_{1}}{P_{d}} \\
& Q_{c}=\frac{\omega_{0} W_{1}}{P_{c}} \\
& Q_{r}=\frac{\omega_{0} W_{1}}{P_{r}}
\end{aligned}
$$

In above expressions, $\mathrm{W}_{1}$ represents total stored energy in the resonator at resonance 
and $\omega_{0}$ is the angular resonant frequency, while $\mathrm{P}_{\mathrm{d}, \mathrm{c}, \mathrm{r}}$ represents power dissipation [45]. When conduction, radiation and external losses are considered negligible then unloaded $\mathrm{Q}$ can be approximated as [32]

$$
Q_{u}=\frac{1}{\tan \delta}
$$

Where $\tan \delta$ is the loss tangent of the resonator. The effect of finite losses in the conducting walls of the resonator determines the unloaded Q factor of the cavity. It can be evaluated by calculating the ratio of volume integral of $E_{y}$ to determine the stored energy to the dissipated energy due to currents in the walls of resonator [53]. The unloaded Q-factor of a ceramic waveguide resonator as shown in Figure 3.1 can be expressed as [20]

$Q_{u}=\frac{1}{\sqrt{\varepsilon_{r}}} \frac{\lambda_{0}}{\delta} \frac{a b d}{4} \frac{\left(p^{2}+q^{2}\right)\left(p^{2}+q^{2}+r^{2}\right)^{\frac{3}{2}}}{a d\left[p^{2} r^{2}+\left(p^{2}+q^{2}\right)^{2}\right]+b d\left[q^{2} r^{2}+\left(p^{2}+q^{2}\right)^{2}\right]+a b r^{2}\left(p^{2}+q^{2}\right)}$

Where $\delta$ is the skin depth and $\lambda_{o}$ is the free space wavelength of the microwave signal passing through the ceramic waveguide cavity and $p=l / a, q=m / b$ and $r=n / d$. Where $l, m, n$ represent half wave variation of electric field along width, height and length of the resonator. For fundamental $\mathrm{TE}_{10}$ mode of ceramic rectangular waveguide $m=0$, thus Equation 3.24 can be simplified as

$$
Q_{u}=\frac{1}{\sqrt{\varepsilon_{r}}} \frac{\lambda_{0}}{\delta} \frac{a b d}{2} \frac{\left(p^{2}+r^{2}\right)^{\frac{3}{2}}}{p^{2} d[a+2 b]+r^{2} a[d+2 b]}
$$

If the resonator length and width are equal i.e. $a=d$ then Equation 3.25 is further simplified as

$$
Q_{u}=\frac{1}{\sqrt{\varepsilon_{r}}} \frac{\lambda_{0}}{\delta} \frac{a b}{2} \frac{\left(p^{2}+r^{2}\right)^{\frac{1}{2}}}{[a+2 b]}
$$

For a rectangular waveguide with the aspect ratio of $\mathrm{b} / \mathrm{a}=0.5$, above Equation 3.26 becomes

$$
Q_{u}=\frac{1}{\sqrt{\varepsilon_{r}}} \frac{\lambda_{0}}{\delta} \frac{a\left(p^{2}+r^{2}\right)^{\frac{1}{2}}}{8}
$$

Where $\frac{\delta}{\lambda_{0}}$ for various polished, corrision free surface metals is given in Table 3-1 [20]. 
Figure 3.4 shows a chart of $Q_{u} \frac{\delta}{\lambda_{0}}$ verses $a / d$ for various aspect ratios $\mathrm{a} / \mathrm{b}$ for the fundamental rectangular waveguide mode $\mathrm{TE}_{10}$.

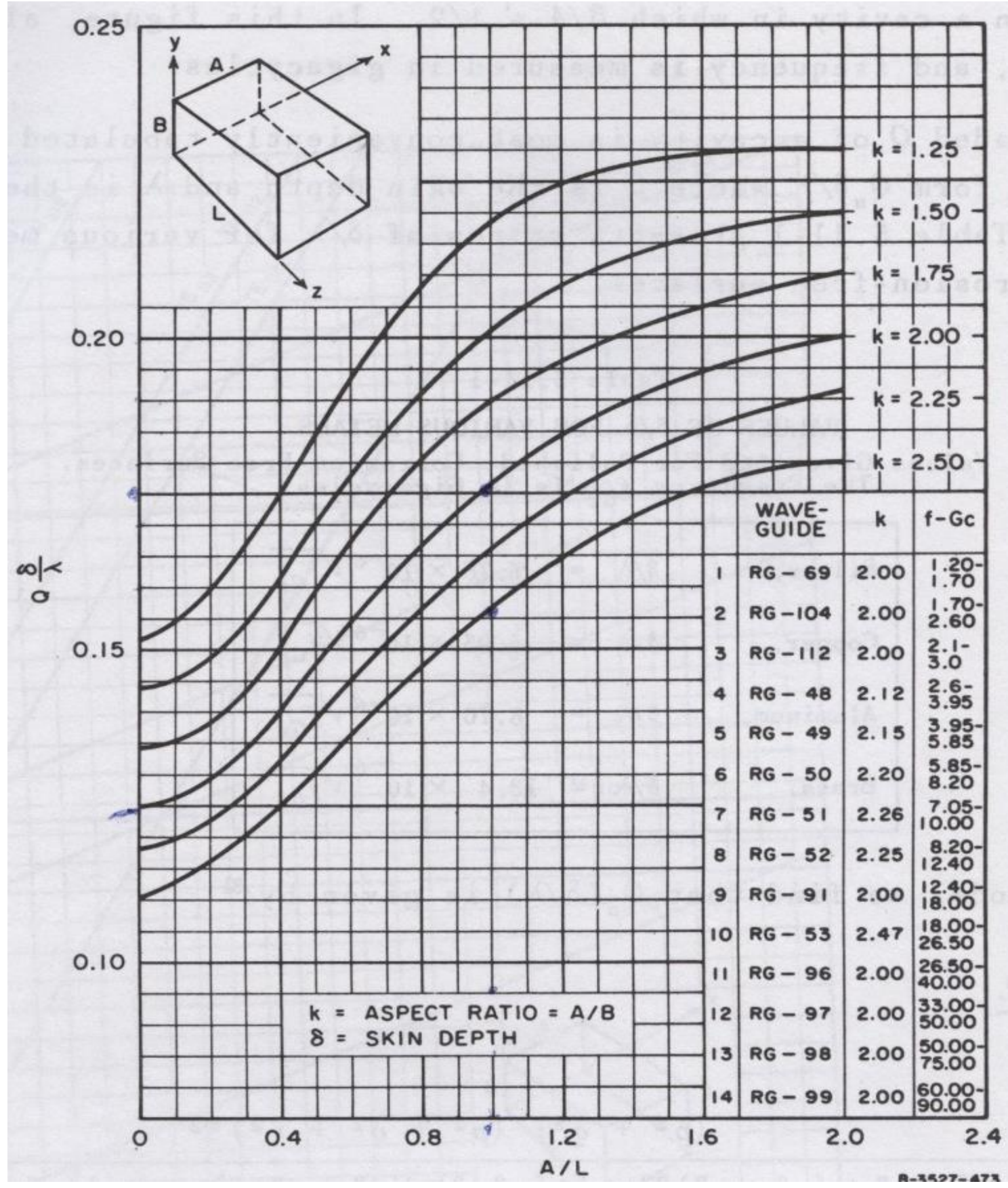

Figure 3.4: Chart for estimating the unloaded $\mathrm{Q}$ of $\mathrm{TE}_{101}$ mode rectangular waveguide resonators [20] 
Table 3-1: $\frac{\delta}{\lambda_{0}}$ values for various metals

\begin{tabular}{|l|l|}
\hline Metal & $\boldsymbol{\delta} / \boldsymbol{\lambda}_{\mathbf{0}}$ \\
\hline Silver & $6.76 \times 10^{-6} \sqrt{f_{G H z}}$ \\
\hline Copper & $6.95 \times 10^{-6} \sqrt{f_{G H z}}$ \\
\hline Aluminium & $8.70 \times 10^{-6} \sqrt{f_{G H z}}$ \\
\hline Brass & $13.4 \times 10^{-6} \sqrt{f_{G H z}}$ \\
\hline
\end{tabular}

A series of simulations is carried out using finite element method (FEM) software tool $\mathrm{HFSS}^{\mathrm{TM}}$ to compare conventional metallic air-filled coaxial resonator and a monolithic dielectric waveguide resonator, $\mathrm{Q}$ versus volume at a resonant frequency of $1 \mathrm{GHz}$. A relative comparison between air-filled coaxial resonator and rectangular dielectric resonator, $\mathrm{Q}$ factor versus volume is given in the Figure 3.5.

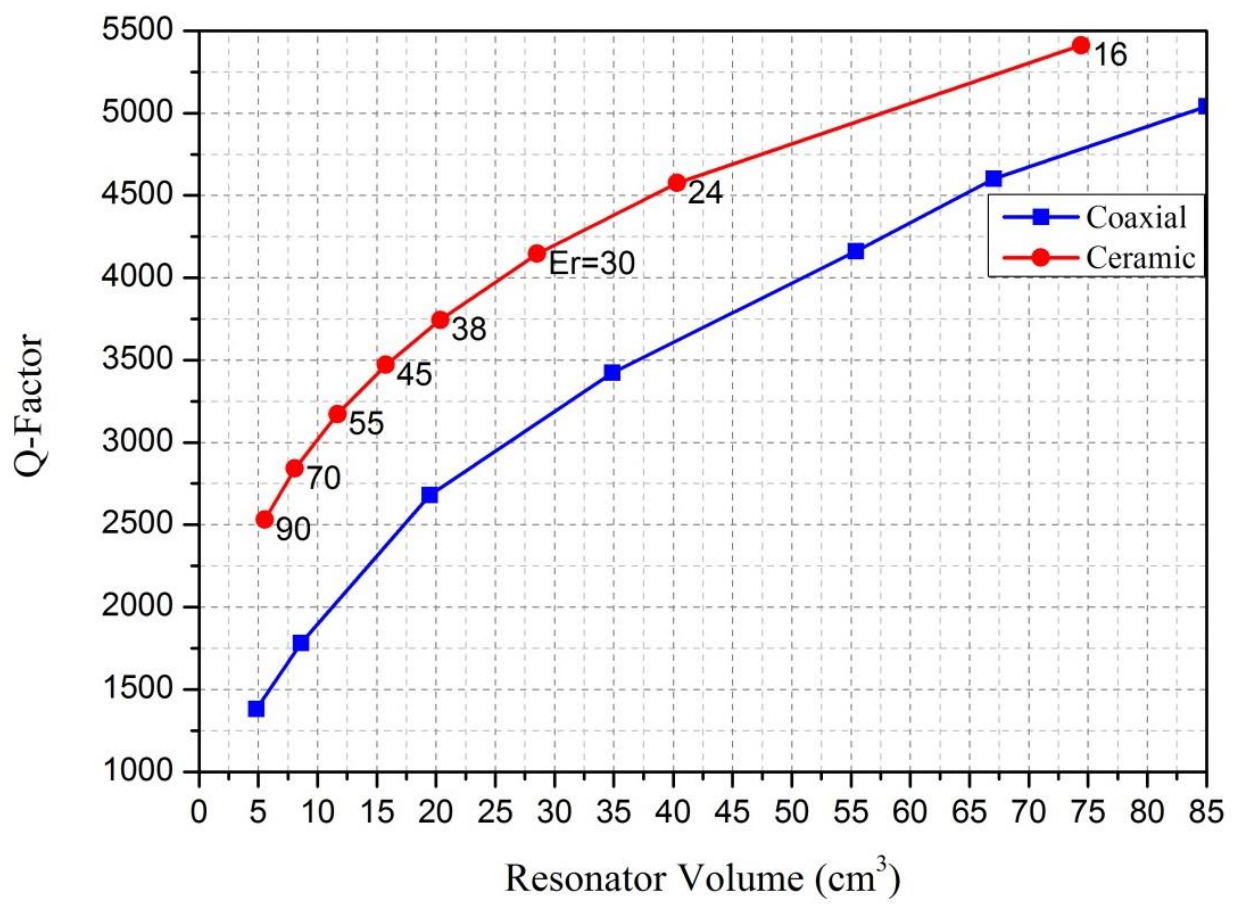

Figure 3.5: Coaxial and ceramic waveguide resonator Q-factor versus volume

It is evident from the graph that for lower volumes, ceramic waveguide resonator offers more than $50 \%$ volume reduction as compared to air-filled coaxial resonator, while maintaining the same Q-factor value. Therefore, significant volume reduction can be 
achieved by using ceramic waveguide resonators to design microwave filters to be used in cellular base stations. Air-filled coaxial resonator consists of various parts such as the lid, the conducting enclosure and the internal metallic rod while ceramic waveguide is an integrated metal coated piece of ceramic material. The ceramic waveguide resonator with an aspect ratio of $\mathrm{b} / \mathrm{a}=0.5$ is used. The $\mathrm{Q}$-factor of the ceramic waveguide resonator can be improved further by increasing its height but the spurious performance of the resonator will be degraded.

\subsection{Spurious performance}

The ceramic rectangular waveguide resonator with an aspect ratio of $b / a=0.5$ reresonates when its narrow dimension becomes equal to cut-off wavelength of $\mathrm{TE}_{20}$ mode. Figure 3.6 shows the spurious performance with respect to height of a ceramic waveguide resonator $\left(\varepsilon_{r}=43\right)$ having its broad dimensions $18 \mathrm{~mm}$ long. The graph suggests that as the waveguide height tends to increase from half of its broad dimension spurious resonance frequency comes closer to the fundamental resonance frequency. When the height of the waveguide resonator equals its width forming a cubic shape it becomes a triple mode resonator at the fundamental frequency.

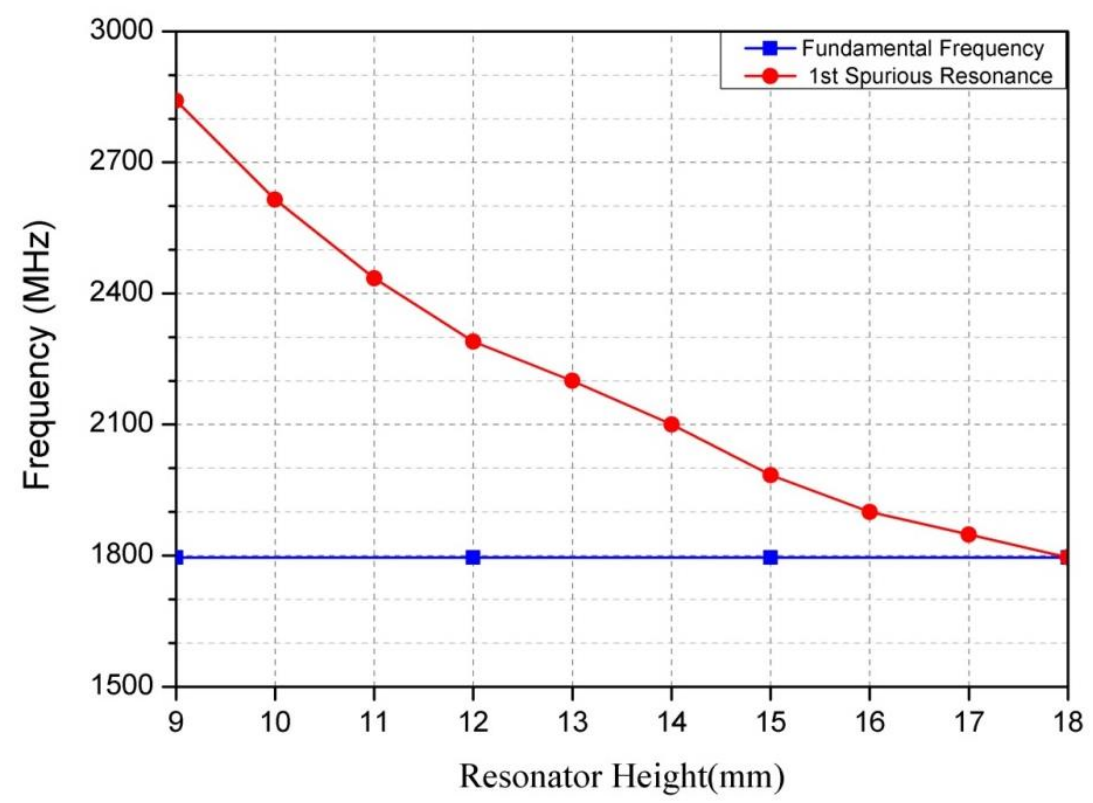

Figure 3.6: Spurious resonance variation with respect to change in the height of ceramic waveguide resonator having $(\mathrm{a}=\mathrm{d}=18 \mathrm{~mm})$ 
Figure 3.7 shows that the Q-factor of the ceramic waveguide resonator is directly proportional to the height of the resonator. Therefore $\mathrm{b} / \mathrm{a}=0.5$ is a good choice to achieve better spurious performance and the Q-factor of the resonator simultaneously.

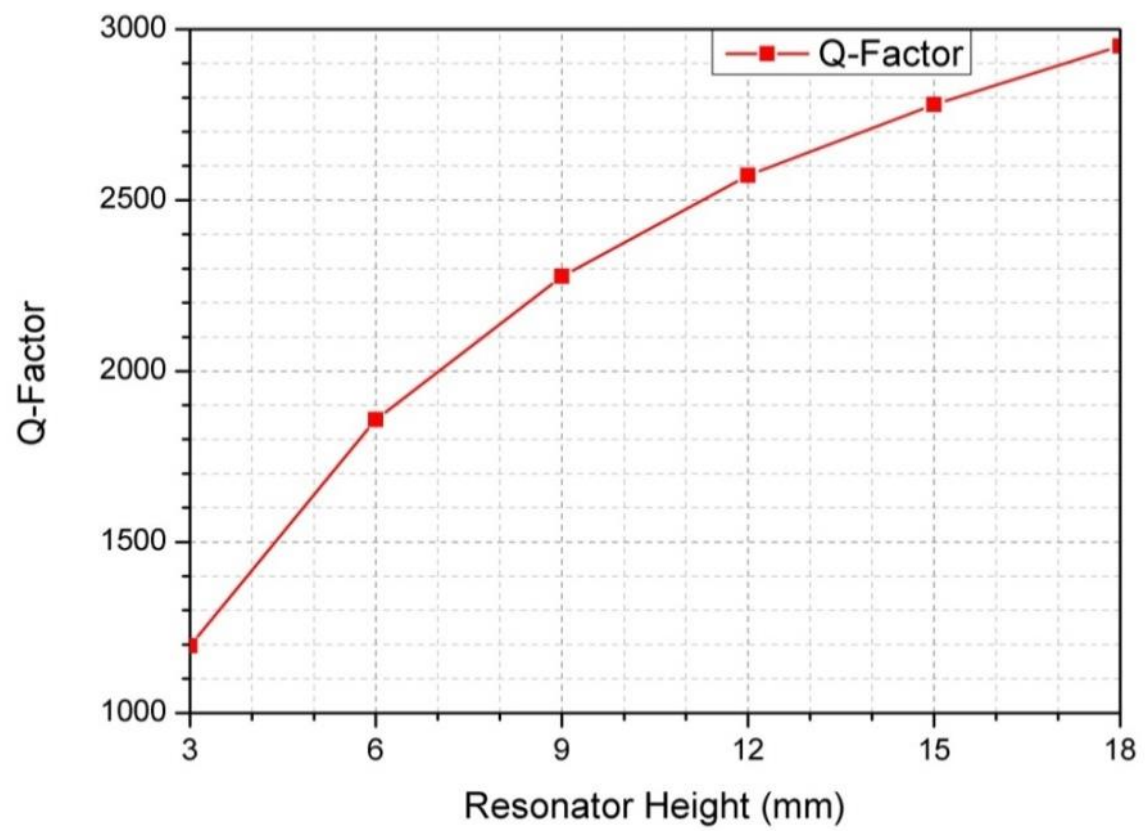

Figure 3.7: Q-factor variation with respect to change in the height of a ceramic waveguide resonator having $(\mathrm{a}=\mathrm{d}=18 \mathrm{~mm})$

\subsection{Resonator tuning methods}

Resonant frequency of the physical resonator can slightly be different from the simulated results due to the manufacturing tolerances, ceramic material inaccuracies and temperature effects. Therefore, it is always preferred to have some kind of tuning mechanism introduced in the resonator for the post-production tuning of the filter. Ceramic waveguide resonators can be tuned in several ways, e.g. by removing metal from the surface of the resonator, introducing a ceramic or metal screw at the centre of the broad wall of the ceramic waveguide resonator, a two way tuning mechanism could be achieved. The most common way of metal tuning screw used to tune air-filled empty waveguide is used to tune the ceramic waveguide resonator [56]. Metal screw is placed at the centre of the broad wall of the resonator so as it can perturb high E-field region of the resonator to achieve more tuning range with the smaller tuning screw diameter. The non-metalized blind hole machined at the centre of the resonator for tuning screw 
placement shifts resonant frequency of the basic waveguide resonator to a higher frequency. Physical layout and its equivalent circuit of the metal tuner to tune the resonant frequency of the ceramic waveguide resonator are shown in Figure 3.8.

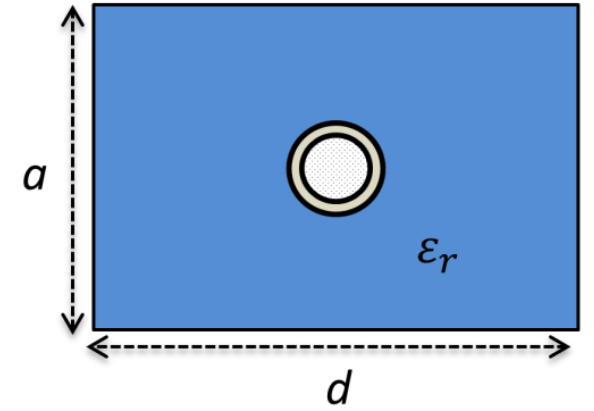

(a)

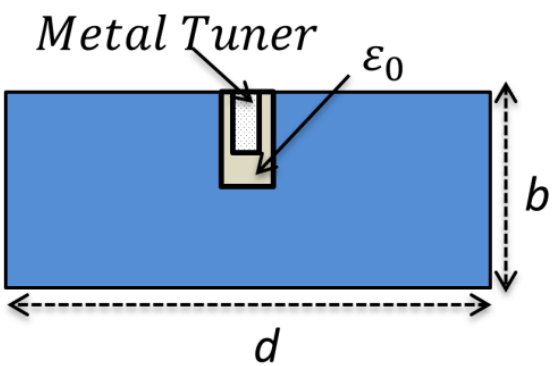

(b)

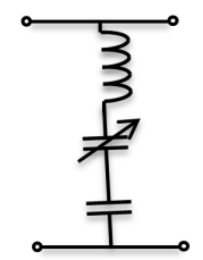

(c)

Figure 3.8: Metal tuner (a) Top view (b) Side view (c) Equivalent circuit

Figure 3.9 shows a tuning range of more than $200 \mathrm{MHz}$ achieved with a metal tuning screw placed in a blind hole of $6 \mathrm{~mm}$ depth, at the centre of the ceramic waveguide resonator broad wall.

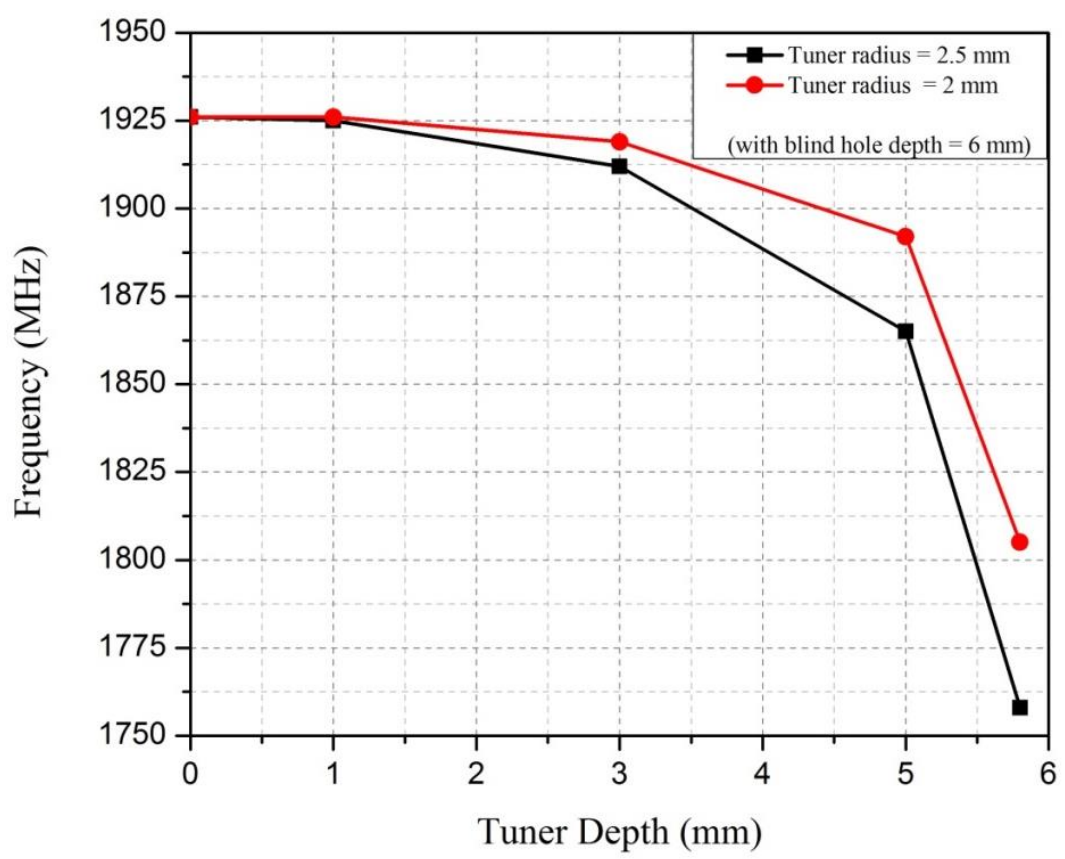

Figure 3.9: Ceramic waveguide resonator tuning with metal screw 
The diameter of the tuner and depth inside the resonator determine the tuning frequency range. The detailed explanation of different tuning mechanism used for ceramic waveguide resonators, tuning range achieved, effect of tuning screw on Q-factor of the resonator and other tuning related issues are discussed in detail in Chapter 8 .

\subsection{High Frequency Structure Simulator $\left(\mathrm{HFSS}^{\mathrm{TM}}\right)$}

$\mathrm{HFSS}^{\mathrm{TM}}$ is a high performance full-wave electromagnetic field simulator to model and analyse arbitrary three dimensional volumetric passive devices using finite element method (FEM). It is used to design several electromagnetic (EM) devices such as antenna, filters, transmission lines and various other three dimensional microwave components. HFSS ${ }^{\mathrm{TM}}$ uses interactive graphical user interface to draw complex 3D structures. It has a built-in library of materials that contains permittivity, permeability and electromagnetic loss tangent information of various commonly used materials. User can add new materials by specifying its properties such as permittivity, permeability and electromagnetic loss tangent. HFSS ${ }^{\mathrm{TM}}$ has multiple solvers based on FEM, integral equation (IE) or hybrid methods to simulate a variety of RF and high speed digital applications. It offers field solving engine with accuracy driven adaptive solutions. It has capability to generate and refine automatic and adaptive mesh and tangential vector finite elements. $\mathrm{HFSS}^{\mathrm{TM}}$ has three solution types named as driven modal, driven terminal and eigenmode.

In a driven mode problem, the modal based S-parameters are calculated by applying the external source of energy at a physical access port of the studied geometry. The scattering matrix solutions are expressed in terms of the incident and reflected powers of waveguide modes at each frequency point in the desired spectrum. Delta-S defined as the maximum change in the magnitude of the S-parameters between two consecutive passes, is the convergence criteria for the modal S-parameters in driven modal solution type. The eigenmodes or resonances of a 3D structure are calculated in sourceless eigenmode solution type of HFSS ${ }^{\mathrm{TM}}$ with defined short circuited planes. This solution type calculates the resonant frequencies and Q-factor of a 3D structure and plots its electromagnetic field patterns at those resonant frequencies. The terminal based Sparameters of multi-conductor transmission line ports are calculated in driven terminal solution type of the HFSS ${ }^{\mathrm{TM}}$. The convergence criteria for driven terminal solution type 
of $\mathrm{HFSS}^{\mathrm{TM}}$ are the delta $\mathrm{S}$ for the single ended or differential nodal S-parameters.

The obvious advantage of $\mathrm{HFSS}^{\mathrm{TM}}$ is to visualize the field patterns of the fundamental mode and spurious resonant modes, thus the simulated structure can be modified to include or remove the spurious modes. The inner material of the structure must be homogenous and linear but can be anisotropic and possess dielectric or conductive losses. The geometry of the analysed structure can be assigned perfect electric or magnetic boundary conditions or can be excited by waveguide or transmission lines depending upon the solution type. The HFSS ${ }^{\mathrm{TM}}$ simulator subdivides geometry of the studied structure into smaller basic elements in the field domain to calculate the actual field pattern. The finite elements are contractual and consists of tetrahedral volume elements in a three dimensional geometry as shown in Figure 3.10. The actual field strength solution is obtained by summing the field strengths at each junction of finite elements.

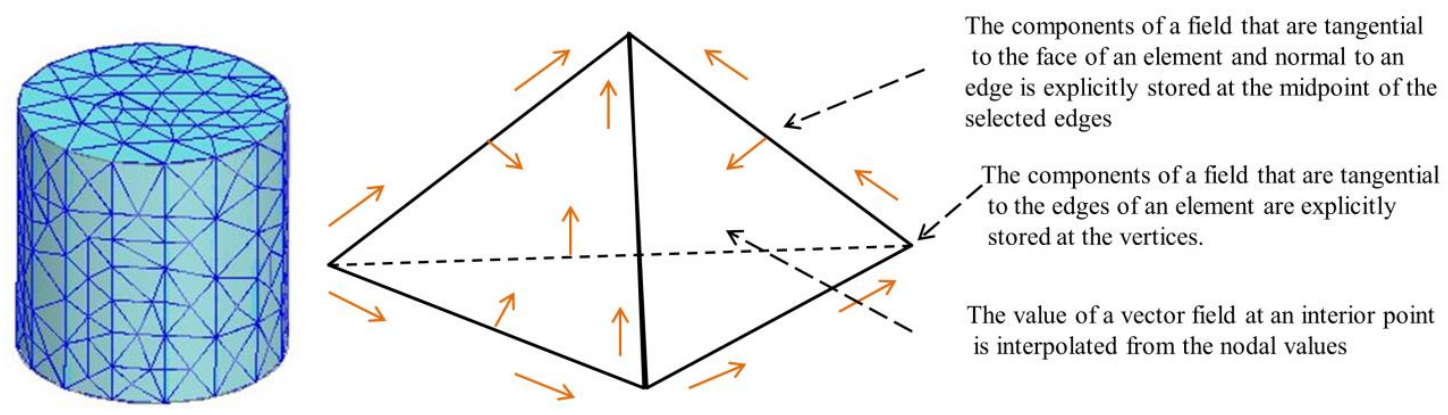

Figure 3.10: Mesh with tetrahedral elements to approximate the shape of a 3D object

HFSS $^{\mathrm{TM}}$ computes the eigenmode resonances of a 3D structure at each port by using the user defined number of modes and S-parameter solutions are obtained within a user defined tolerance. The refinement of the finite element mesh continues until the allowed tolerance level is achieved. An adaptive mesh is run to create a finite element mesh at the desired frequency and then the frequency sweep is added to obtain the S-parameters for the desired range of frequencies. For narrowband applications the adaptive pass is usually run at the centre frequency of the desired spectrum. The mesh algorithm helps to predict the required mesh density for particular boundary configurations. The resolution of the mesh can be increased to improve the accuracy of the design at the expense of increased simulation times. Also to ensure the convergence of each adaptive mesh a 
convergence value is required that also is a trade-off between computational time and accuracy of the design. An example of a ceramic waveguide resonator with different mesh resolution is shown in Figure 3.11.

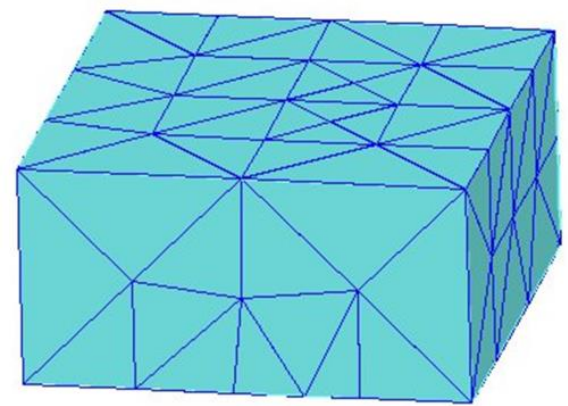

(a)

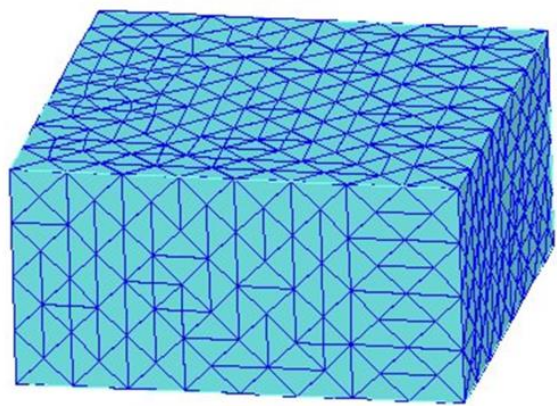

(b)

Figure 3.11: Finite element method analysis of ceramic waveguide with different mesh resolutions (a) $0.6 \lambda$ mesh (b) $0.2 \lambda$ mesh

The HFSS ${ }^{\mathrm{TM}}$ simulator calculates the electromagnetic fields of a 3D structure with high level of accuracy by using FEM method. There are some limitations in HFSS ${ }^{\mathrm{TM}}$ such as it does not calculate the impedances unless a voltage integration line is defined on the ports. These lines connect the maximum voltage difference points at each port and are different for each individual mode. The computational time and high memory requirement are other limitations of the $\mathrm{HFSS}^{\mathrm{TM}}$ simulation tool.

\subsection{Summary}

A ceramic waveguide resonator is explained in this Chapter. The physical dimensions and Q-factor of a metal coated ceramic waveguide resonator decreases by a factor of $\frac{1}{\sqrt{\varepsilon_{r}}}$ as compared to air-filled empty waveguide cavity. The ceramic waveguide resonator offers more than $50 \%$ volume reduction as compared to the conventional air filled coaxial resonators while maintaining the same electrical performance. $\mathrm{TE}_{10}$ is the fundamental mode of the rectangular ceramic waveguide resonator. The first spurious resonance occurs almost at double the fundamental frequency of the resonator. The Qfactor of the resonator can be increased by increasing the aspect ratio b/a, but at the expense of poor spurious performance. The ceramic waveguide resonator can be tuned 
by placing a metal screw at the centre of the broad wall of the resonator. The proposed ceramic waveguide resonator is used to design integrated ceramic waveguide filters discussed in upcoming Chapters of the thesis. 


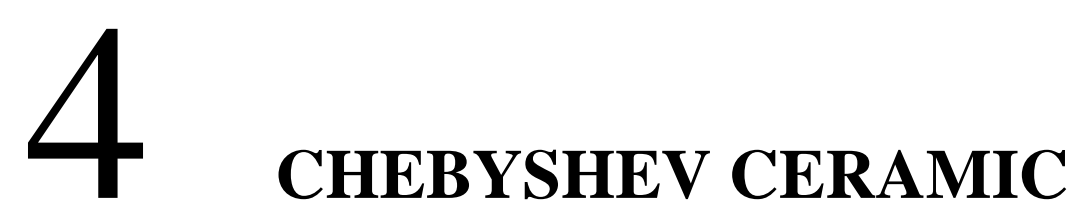 WAVEGUIDE BANDPASS FILTER}

\subsection{Introduction}

An integrated ceramic waveguide resonator filter design is presented in this Chapter. The volume of a ceramic waveguide cavity with permittivity $\varepsilon_{r}$ decreases $\frac{1}{\varepsilon_{r} \sqrt{\varepsilon_{r}}}$ times as compared to air-filled waveguide resonator [49]. For a given value of Q-factor, ceramic waveguide resonator occupies less than half of the conventional coaxial resonator volume used in cellular base station filters. A sixth order Chebyshev filter is designed using ceramic rectangular waveguide resonators. Metal coated through holes placed at the broad wall of the waveguide are used to control the amount of inter resonator coupling extracted from the coupling coefficients of the lumped element equivalent filter. Ceramic waveguide filter is excited using $50 \Omega$ coaxial probes. Input/output probes are directly placed at the centre of the broad wall of the first and last resonator section of the filter. Probe position from the shorted back end and its depth inside the ceramic waveguide are the input variables to adjust the external coupling amount. The filter is coated with a metal ink except the input/output probe positions. The fabricated filter results are compared against EM simulated response of the filter. Practical filter response is in good agreement to the EM simulation except high insertion loss in the passband of the filter, which is mainly caused owing to leakage from input and output probes. 


\subsection{GSM 1800 filter specifications}

A Chebyshev transfer function approximation is chosen to design a bandpass filter composed of ceramic waveguide resonators.

$$
\left|S_{21}(j \omega)\right|^{2}=\frac{1}{1+\varepsilon^{2} T_{n}^{2}(\omega)}
$$

The specifications for the filter to be designed are as follows.

Table 4-1: Specifications for the bandpass ceramic waveguide filter

\begin{tabular}{|l|l|}
\hline Centre Frequency $\left(\mathrm{f}_{0}\right)$ & $1842.5 \mathrm{MHz}$ \\
\hline Passband Bandwidth & $75 \mathrm{MHz}$ \\
\hline Out of Band Rejection & $>50 \mathrm{~dB}$ at $\mathrm{f}<1750 \mathrm{MHz}$ \\
& $>50 \mathrm{~dB}$ at $\mathrm{f}>1930 \mathrm{MHz}$ \\
\hline Passband Return Loss $\left(L_{R}\right)$ & $>20 \mathrm{~dB}$ \\
\hline Passband Insertion Loss $(\mathrm{IL})$ & $<0.7 \mathrm{~dB}$ \\
\hline System Impedance $\left(\mathrm{z}_{0}\right)$ & $50 \Omega$ \\
\hline
\end{tabular}

\subsection{Lumped element Chebyshev filter design}

The ripple level in the passband of the Chebyshev filter for $20 \mathrm{~dB}$ return loss $\left(L_{R}\right)$ in the passband is calculated using Equation 2.19

$$
\varepsilon=0.1005
$$

The order of the filter required to meet the specification is calculated using Equation 2.23

$$
N \geq 5.98
$$

The doubly terminated normalised lowpass prototype filter element values $\left(g_{i}\right)$ can be calculated from the following relationships given as [20]

$$
g_{1}=\frac{2 a_{1}}{\gamma}
$$




$$
\begin{aligned}
g_{i} & =\frac{4 a_{i-1} a_{i}}{b_{i-1} g_{i-1}}, \quad i=2,3 \ldots . N \\
g_{N+1} & =1 \text { for } N \text { odd } \\
& =\operatorname{coth}^{2}\left(\frac{\beta}{4}\right) \text { for } N \text { even }
\end{aligned}
$$

Where

$$
\begin{gathered}
\beta=\ln \left(\operatorname{coth} \frac{L_{R}}{17.37}\right) \\
\gamma=\sinh \left(\frac{\beta}{2 N}\right) \\
a_{i}=\sin \left[\frac{(2 i-1) \pi}{2 N}\right], i=1,2 \ldots N
\end{gathered}
$$

And

$$
b_{i}=\gamma^{2}+\sin ^{2}\left(\frac{i \pi}{N}\right), i=1,2 \ldots N
$$

Therefore, the calculated element values of a sixth order Chebyshev filter are given as from Equations 4.2, 4.3 and 4.4

$$
\begin{aligned}
& g_{0}=1 \\
& g_{1}=1.1681 \\
& g_{2}=1.4039 \\
& g_{3}=2.0562 \\
& g_{4}=1.5170 \\
& g_{5}=1.9029 \\
& g_{6}=0.8618 \\
& g_{7}=1.3554
\end{aligned}
$$

The normalised external couplings coefficients are calculated as

$$
\begin{gathered}
k_{e}=\frac{1}{\sqrt{g_{0} g_{1}}}=\frac{1}{\sqrt{g_{N, N+1}}} \\
=0.9253
\end{gathered}
$$


And the internal couplings are calculated as

$$
k_{i, i+1}=\frac{1}{\sqrt{g_{i} g_{i+1}}} i=1,2 \ldots N-1
$$

This gives,

$k_{12}=k_{56}=0.7809$

$k_{23}=k_{45}=0.5886$

$k_{34}=0.5662$

The normalised coupling coefficient can be represented in terms of coupling bandwidths i.e

$$
K_{i, i+1}(G H z)=\frac{1}{\sqrt{g_{i} g_{i+1}}} * \text { Bandwidth }(G H z)
$$

The bandwidth of the filter to be designed is $0.075 \mathrm{GHz}$, Therefore, the coupling coefficients in terms of coupling bandwidths become,

$K_{e}=0.0694 \mathrm{GHz}$

$K_{12}=K_{56}=0.0586 \mathrm{GHz}$

$K_{23}=K_{45}=0.0441 \mathrm{GHz}$

$K_{34}=0.0425 \mathrm{GHz}$

The inductor used to realize external impedance inverter of the bandpass filter can be calculated from the relation [57]

$$
\begin{gathered}
L_{e}=\frac{Z_{0}}{\pi \sqrt{2 \pi f_{0(G H z)} K_{e(G H z)}}} n H \\
=17.756 n H
\end{gathered}
$$

And the inductor used to form an impedance inverter between adjacent resonators of the bandpass filter as shown in Figure 4.1 can be obtained from the relation [57].

$$
L_{i j}=\frac{Z_{0}}{\pi^{2} K_{i j}(G H z)} n H
$$


This gives,

$$
\begin{aligned}
& L_{12}=L_{56}=86.4505 n H \\
& L_{23}=L_{45}=114.875 n H \\
& L_{34}=119.201 n H
\end{aligned}
$$
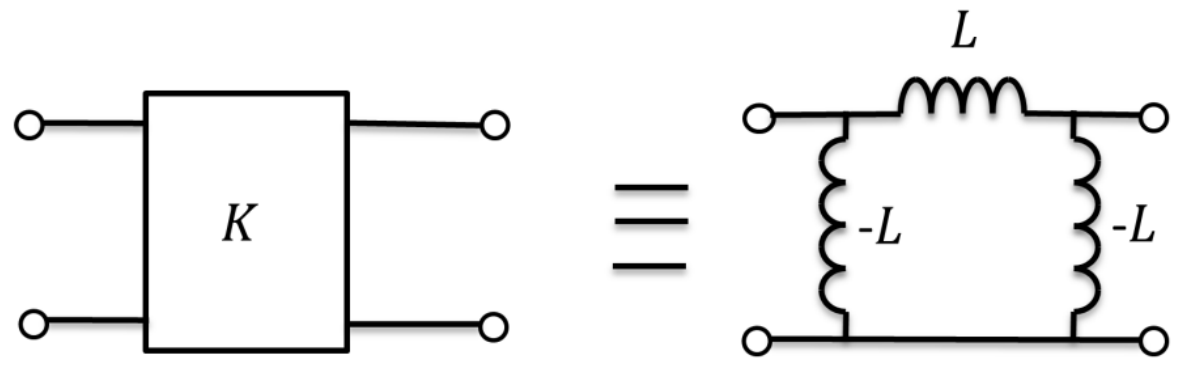

Figure 4.1: Impedance inverter equivalent circuit

The element values of shunt resonator with centre frequency $f_{0}=1.8425 \mathrm{GHz}$ and system impedance level of $Z_{0}=50 \mathrm{ohm}$ can be calculated as from [7]

$$
\begin{aligned}
C= & \frac{1}{4 f_{0(G H z)} Z_{0}} n F \\
= & 2.713 p F
\end{aligned}
$$

And

$$
\begin{aligned}
L= & \frac{Z_{0}}{\pi^{2} f_{0(G H z)}} n H \\
= & 2.75 n H
\end{aligned}
$$

Figure 4.2 shows the layout of a sixth order inverter coupled Chebyshev bandpass filter. Where each shunt resonator is composed of a parallel $L C$ resonant circuit with $f_{0}=$ $18425 \mathrm{MHz}$. 


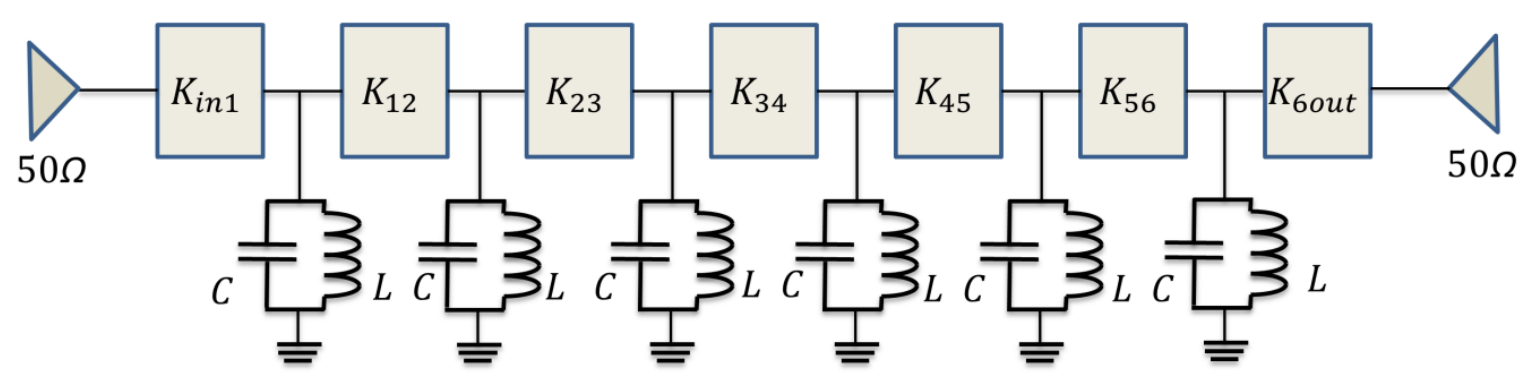

Figure 4.2: Inverter coupled bandpass filter layout

Figure 4.3 shows the response of a sixth order Chebyshev filter composed of inverter coupled shunt resonators. The passband insertion loss of the filter is less than $0.7 \mathrm{~dB}$, when an unloaded Q-factor of resonators is selected to be 2400 .

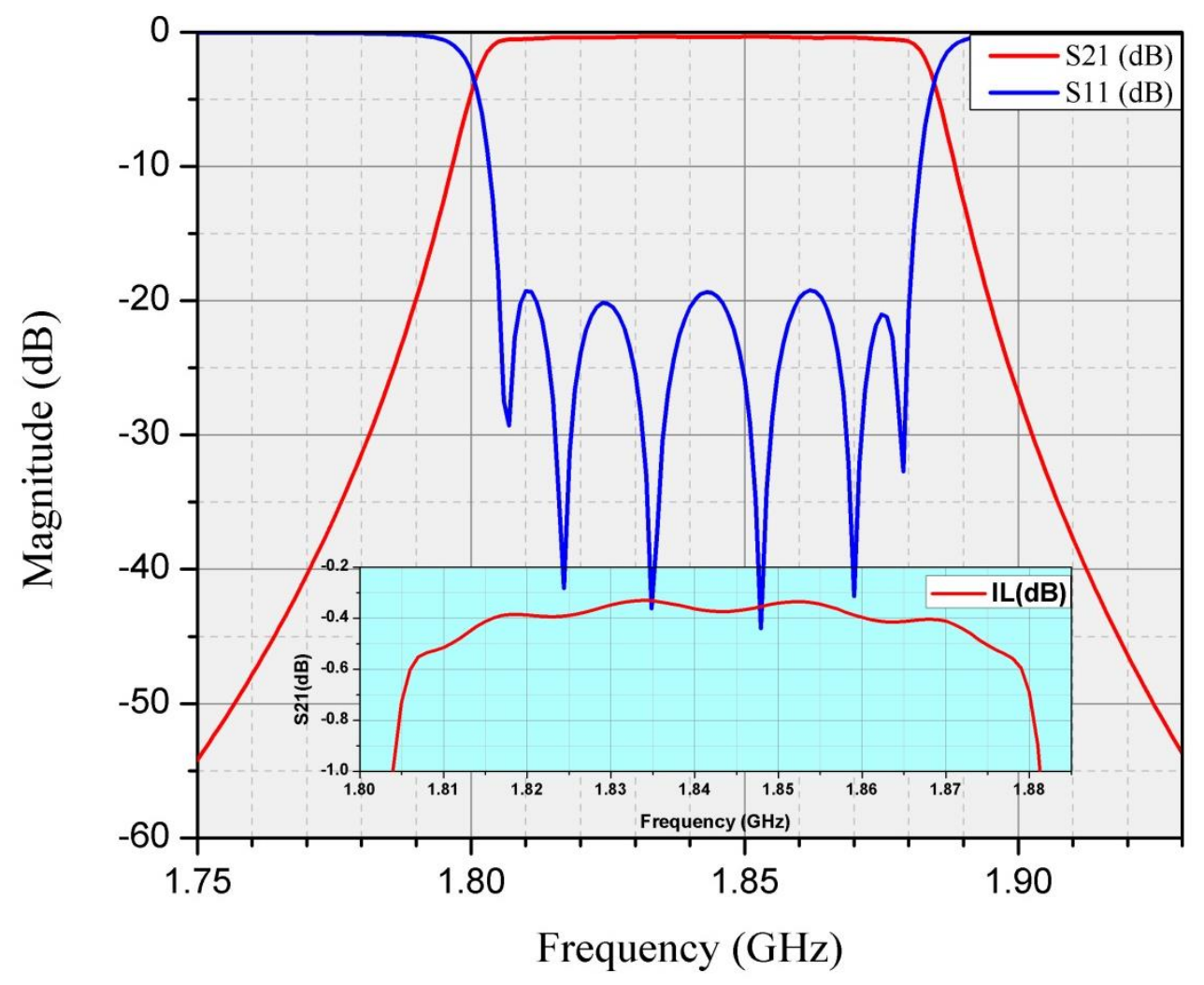

Figure 4.3: Inverter coupled lumped element bandpass filter response

\subsection{Ceramic waveguide resonator design}

A ceramic waveguide resonator is a silver coated half guide wavelength long 
rectangular waveguide structure composed of barium-titanate $\left(\mathrm{BaTio}_{3}\right)$ ceramic material with relative permittivity of 45 and dielectric loss tangent of 0.00004 . The silver ink used to coat the ceramic structure has the conductivity of $4.4 \times 10^{7} \Omega^{-1} \mathrm{~m}^{-1}$. The EM simulations show a Q-factor of 2400 at a frequency of $1.842 \mathrm{GHz}$. The dimensions of the ceramic waveguide resonator are calculated based on the equation for fundamental mode $\left(\mathrm{TE}_{10}\right)$ resonance of ceramic waveguide resonator given in Equation 3.1

For a rectangular ceramic waveguide resonator with an aspect ratio of $\frac{b}{a}=0.5$, by putting $a=d=2 b, f_{0}=1842.5 \mathrm{MHz}$ and $\varepsilon_{r}=45$ in Equation 3.1, we get

$$
a=d=2 b=17.15 \mathrm{~mm}
$$

Therefore, the silver coated ceramic waveguide resonator with an aspect ratio of 0.5 has the dimensions of $17.15 \times 17.15 \times 8.58(\mathrm{~mm})$ to resonate at a fundamental frequency of 1842.5 MHz. The effective wavelength of the wave in the ceramic material with a dielectric constant of 45 at a frequency of $1842 \mathrm{MHz}$ becomes,

$$
\begin{gathered}
\lambda_{d}=\frac{\lambda_{0}}{\sqrt{\varepsilon_{r}}} \\
=\frac{162.8}{\sqrt{45}}=24.30 \mathrm{~mm}
\end{gathered}
$$

Where

$$
\begin{gathered}
\lambda_{0}=\frac{c}{f} \\
=\frac{3 \times 10^{8}}{1842.5 \times 10^{6}}=162.8 \mathrm{~mm}
\end{gathered}
$$

The cut-off wavelength of the ceramic waveguide resonator can be calculated as [3]

$$
\begin{gathered}
\lambda_{c}=2 a \\
=34.3 \mathrm{~mm}
\end{gathered}
$$

Hence the cut-off frequency of the rectangular ceramic waveguide with its broad dimension equals to $a$ can be calculated as [3]

$$
\begin{aligned}
\omega_{c} & =\frac{\pi v}{a}=\frac{\pi}{a \sqrt{\mu_{0} \varepsilon_{0} \varepsilon_{r}}} \\
& =8.1865 \times 10^{9}
\end{aligned}
$$


Therefore,

$$
\begin{gathered}
f_{C}=\frac{\omega_{c}}{2 \pi} \\
=1.3029 \mathrm{GHz}
\end{gathered}
$$

Figure 4.4 show the E-field pattern of a ceramic waveguide resonator simulated using a 3D EM simulation tool $\mathrm{HFSS}^{\mathrm{TM}}$. It is clear that E-field in a rectangular ceramic waveguide resonator makes a half wave variation along the width and length of the resonator while there is no E-field variation along the height of the resonator. E-field of the resonator vanishes completely at $\mathrm{x}=0$ and $\mathrm{x}=\mathrm{d}$ which confirms that the resonator is half wavelength long in both of its broad dimensions. The centre of the resonator is the region with maximum E-field and the weakest E-field lines occur near the side walls of the cavity. Figure 4.5 shows the plot of $\mathrm{H}$-field variations in a rectangular ceramic waveguide resonator. It is obvious that $\mathrm{H}$-field makes horizontal circles around the $\mathrm{E}$ field lines and repeat itself every half wavelength variation. The maximum H-field strength occurs at the midpoint of each wall of ceramic rectangular waveguide cavity. And its minima occur at the centre of resonator cavity.
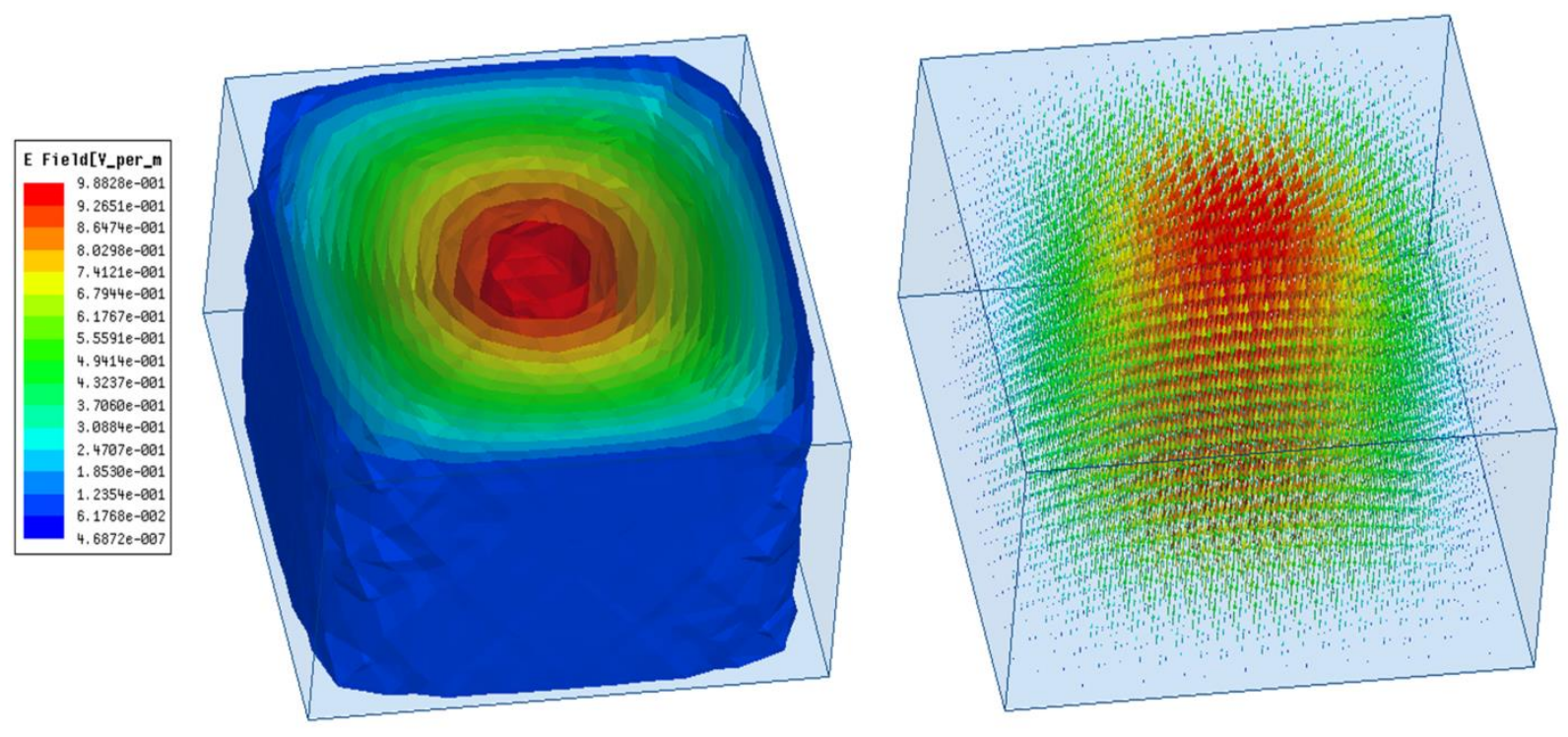

Figure 4.4: E-field distribution of ceramic waveguide resonator (Magnitude \& Vector) 

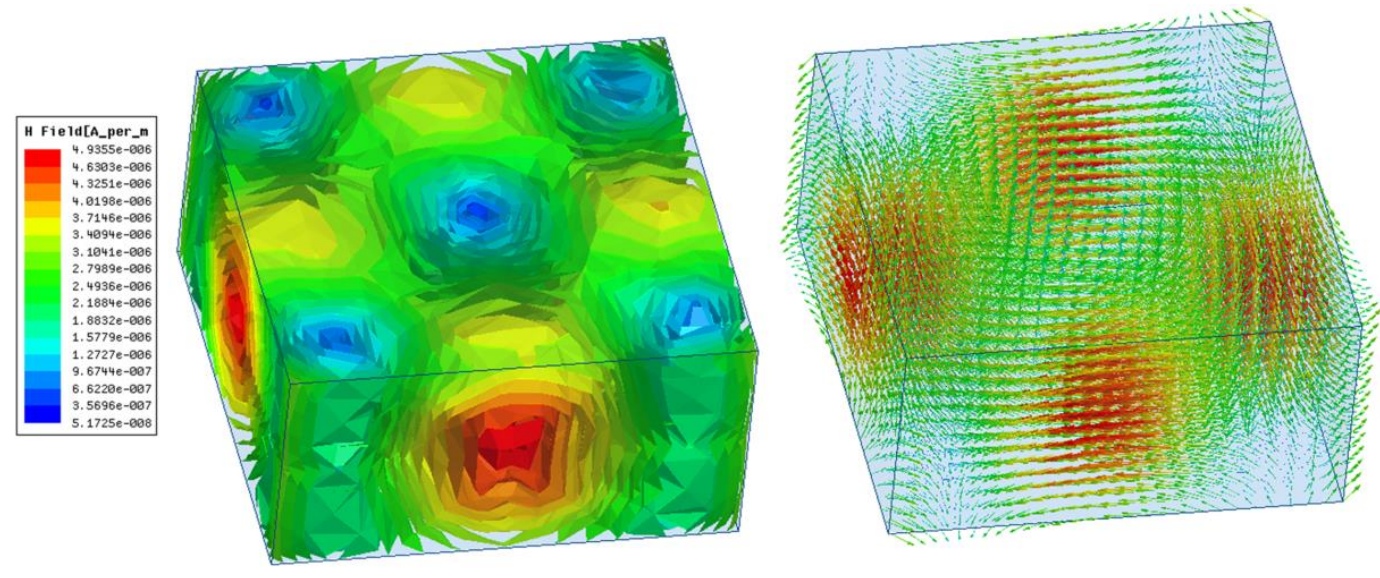

Figure 4.5: H-field distribution of ceramic waveguide resonator (Magnitude \& Vector)

The Q-factor for the $\mathrm{TE}_{10}$ mode of a rectangular ceramic waveguide can be computed from the relation given as [53]

$$
Q_{u}=\frac{1}{\sqrt{\varepsilon_{r}}} \frac{\lambda}{\delta} \frac{a b d}{2} \frac{\left(\frac{1}{a^{2}}+\frac{1}{d^{2}}\right)^{\frac{3}{2}}}{\frac{d}{a^{2}}(a+2 b)+\frac{a}{d}(d+2 b)}
$$

By putting $a=d$ and $b=\frac{a}{2}$, we get

$$
Q_{u}=\frac{0.17677}{\sqrt{\varepsilon_{r}}} \frac{\lambda}{\delta}
$$

For silver coating with a conductivity of $5.8 \times 10^{7} \Omega^{-1} \mathrm{~m}^{-1}$ [3],

$$
\frac{\lambda}{\delta}=\frac{1.479 \times 10^{5}}{\sqrt{f_{G H z}}}
$$

Therefore, the calculated unloaded Q-factor of a rectangular ceramic waveguide resonator at a frequency of $1842.5 \mathrm{MHz}$ was 2871 . By including the effect of dielectric loss tangent of the material i.e. $a n \delta=0.00004$, we get

$$
\begin{aligned}
Q_{\text {total }} & =\left(\frac{1}{Q_{u}}+\frac{1}{Q_{L}}\right)^{-1} \\
& =2575
\end{aligned}
$$

The HFSS ${ }^{\mathrm{TM}}$ simulated Q-factor value was 2400 which is mainly because a more practical value of silver conductivity i.e. $4.4 \times 10^{7} \Omega^{-1} \mathrm{~m}^{-1}$ was used in simulation. Table 
4-2 shows the fundamental resonance frequency, first spurious resonance and the Qfactor values of the ceramic waveguide resonator obtained through $\operatorname{HFSS}^{\mathrm{TM}}$ simulation. It is observed that the first spurious resonance occurs at $2913 \mathrm{MHz}$, thus providing a wide spurious free stopband bandwidth.

Table 4-2: Ceramic WG resonator eigenmode data $\left(\mathrm{HFSS}^{\mathrm{TM}}\right)$

\begin{tabular}{|c|c|c|}
\hline Mode Number & Frequency (MHz) & Q-Factor \\
\hline 01 & 1842.5 & 2400 \\
\hline 02 & 2913.3 & 2700 \\
\hline
\end{tabular}

\subsection{Inter resonator coupling}

For a rectangular ceramic block, inter resonator coupling can be achieved by circular through holes placed in the broad dimension of the waveguide at half guided wavelength intervals. Circular holes or inductive posts behave as impedance inverters over a broad bandwidth of the filter [3]. A circular hole can be modelled as a shunt inductor behaving as impedance inverter between half wavelength resonator sections. Once the susceptance (B) of the impedance inverter is calculated, the number of holes and their diameter can be calculated easily from the graphs available in [58]. Adjustment in inverter susceptances can be achieved with fixed number of holes and fixed diameter by varying the distance among them. The coupling holes are placed symmetrically across the waveguide broad dimension in order to suppress the higher order modes [3]. Figure 4.6 shows a circular inductive hole in ceramic waveguide and its equivalent electrical circuit.
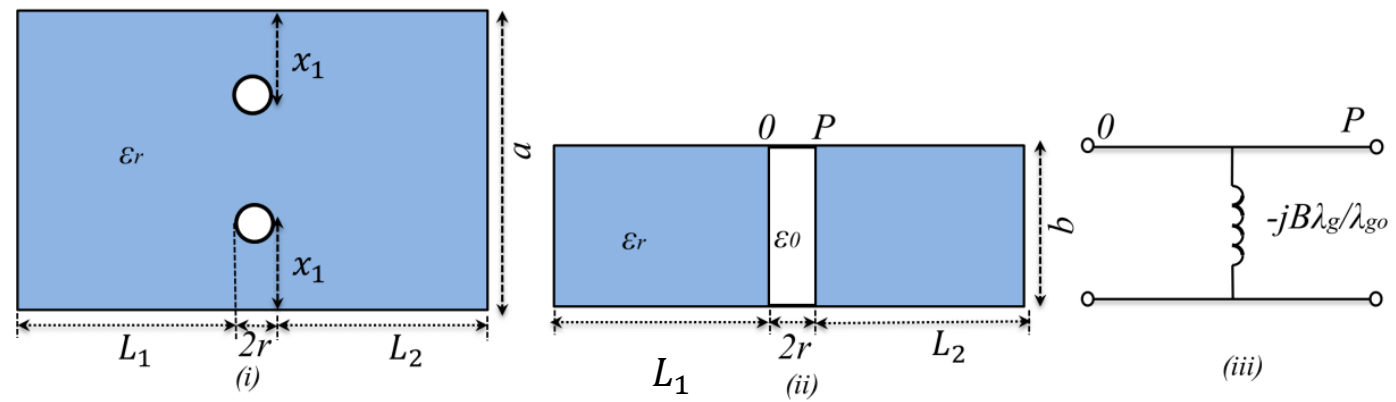

Figure 4.6: Through hole in ceramic (i) Top view (ii) Side view (iii) Equivalent circuit 
Figure 4.7 shows the different coupling susceptance values achieved as a function of radii of post and their offset from side wall of the waveguide.
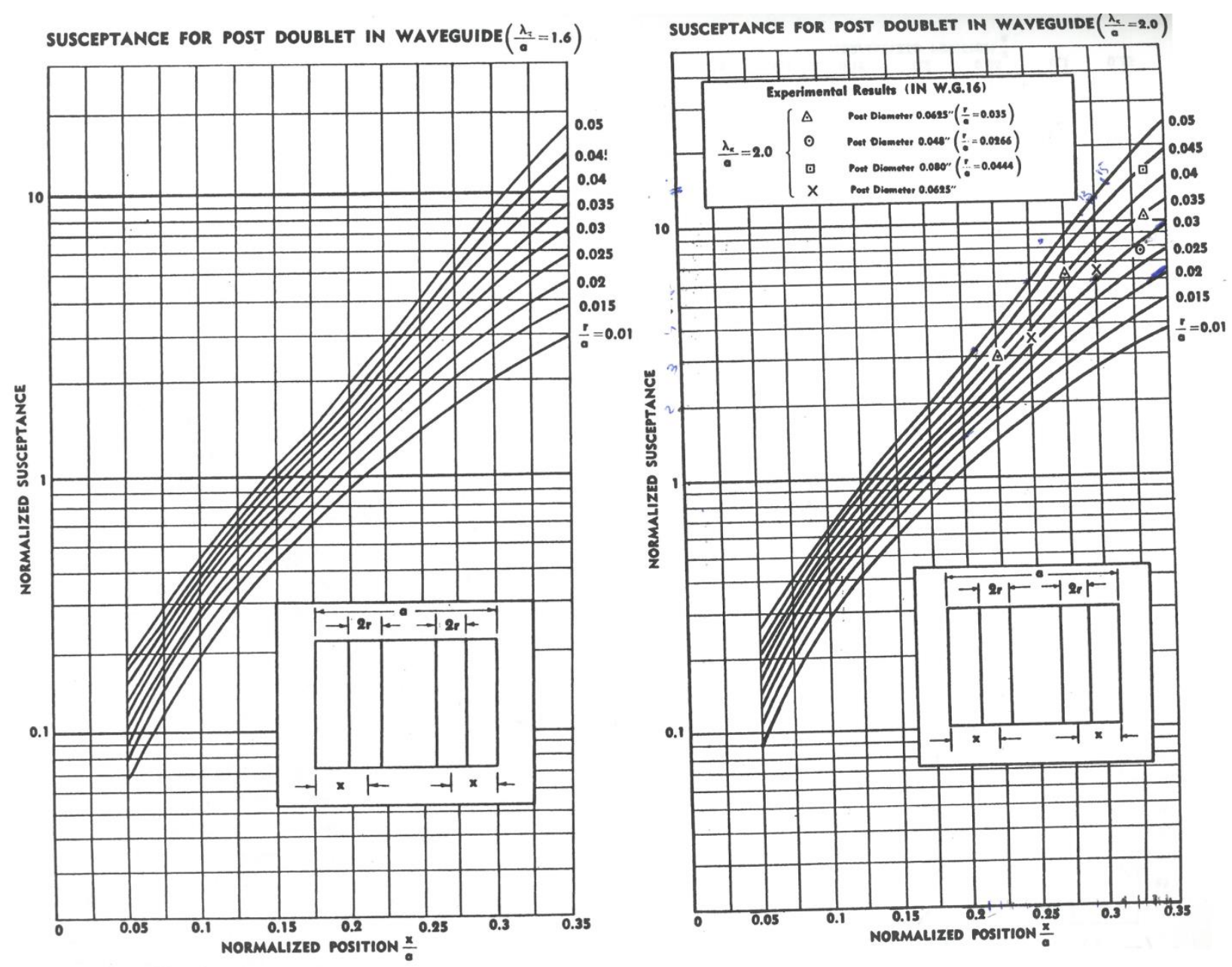

Figure 4.7 : Normalized susceptance for two posts in a waveguide [58]

A waveguide section can be represented by its transfer matrix which is that of a transmission line with frequency dependent propagation constant and characteristic impedance, i.e. [3]

$$
Z_{0}=\frac{\eta_{0} \lambda_{g}}{\lambda_{0}}=\frac{\eta_{0}}{\left[1-\left(\frac{\omega_{c}}{\omega}\right)^{2}\right]^{\frac{1}{2}}}
$$

And 


$$
\beta=\frac{2 \pi}{\lambda_{g}}=\frac{\omega}{v}\left[1-\left(\frac{\omega_{c}}{\omega}\right)^{2}\right]^{\frac{1}{2}}
$$

The transfer matrix for a length of waveguide with characteristic impedance $\left(\mathrm{Z}_{0}\right)$ and electrical length $\theta$ can be written in the matrix form as

$$
\left[\begin{array}{ll}
A_{A B C D} & B_{A B C D} \\
C_{A B C D} & D_{A B C D}
\end{array}\right]=\left[\begin{array}{ll}
\cos \theta & j \sin \theta \\
j \sin \theta & \cos \theta
\end{array}\right]
$$

Where

$$
\theta=\beta d=\frac{2 \pi d}{\lambda_{g}}
$$

Or

$$
\theta=\frac{\pi \lambda_{g 0}}{\lambda_{g}}
$$

Where $\lambda_{g 0}$ is chosen to be the guide wavelength when the waveguide is half wavelength long i.e.

$$
d=\frac{\lambda_{g 0}}{2}
$$

The discontinuities can be introduced in several ways in waveguide in order to realize impedance inverters. The easiest way is to load the waveguide with shunt inductive discontinuities by introducing shorted through holes in the broad wall of the ceramic waveguide. The equivalent circuit of a shunt discontinuity in a waveguide consists of shunt inductor is shown in Figure 4.6. The transfer matrix of the shunt inductor is given as

$$
[T]=\left[\begin{array}{cc}
1 & 0 \\
-\frac{j B \lambda_{g}}{\lambda_{g 0}} & 1
\end{array}\right]
$$

The reference planes $\mathrm{O}$ and $\mathrm{P}$ are normally within the diameter of the shorted hole in ceramic waveguide. The parameter B and the reference plane locations are determined by the number of holes and their diameter.

The inductive iris section is further modified by symmetrically embedding in a uniform 
section of waveguide of electrical length given by Equation 4.32 as shown in Figure 4.8

$$
\psi=\frac{\psi_{0} \lambda_{g 0}}{\lambda_{g}}
$$

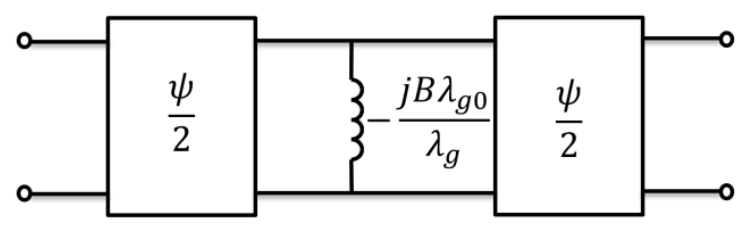

Figure 4.8: Shunt inductive iris embedded in a waveguide section

The transfer matrix of the new embedded section becomes

$$
\begin{aligned}
{\left[\begin{array}{ll}
A_{A B C D} & B_{A B C D} \\
C_{A B C D} & D_{A B C D}
\end{array}\right] } \\
=\left[\begin{array}{cc}
\cos \left(\frac{\psi}{2}\right) & j \sin \left(\frac{\psi}{2}\right) \\
j \sin \left(\frac{\psi}{2}\right) & \cos \left(\frac{\psi}{2}\right)
\end{array}\right]\left[\begin{array}{cc}
1 & 0 \\
j B \lambda_{g} & 1 \\
\lambda_{g 0} & 1
\end{array}\right]\left[\begin{array}{cc}
\cos \left(\frac{\psi}{2}\right) & j \sin \left(\frac{\psi}{2}\right) \\
j \sin \left(\frac{\psi}{2}\right) & \cos \left(\frac{\psi}{2}\right)
\end{array}\right] \\
=\left[\begin{array}{cc}
\cos \left(\frac{\psi}{2}\right)+\frac{B \lambda_{g}}{\lambda_{g 0}} \sin \left(\frac{\psi}{2}\right) & j \sin \left(\frac{\psi}{2}\right) \\
j \sin \left(\frac{\psi}{2}\right)-\frac{j B \lambda_{g}}{\lambda_{g 0}} \cos \left(\frac{\psi}{2}\right) & \cos \left(\frac{\psi}{2}\right)
\end{array}\right]\left[\begin{array}{ll}
\cos \left(\frac{\psi}{2}\right) & j \sin \left(\frac{\psi}{2}\right) \\
j \sin \left(\frac{\psi}{2}\right) & \cos \left(\frac{\psi}{2}\right)
\end{array}\right] \\
=\left[\begin{array}{ll}
\cos (\psi)+\frac{B \lambda_{g}}{2 \lambda_{g 0}}+\sin (\psi) & j\left[\sin (\psi)+\frac{B \lambda_{g}}{\lambda_{g 0}} \sin ^{2}\left(\frac{\psi}{2}\right)\right] \\
j\left[\sin (\psi)-\frac{B \lambda_{g}}{\lambda_{g 0}} \cos ^{2}\left(\frac{\psi}{2}\right)\right] & \cos (\psi)+\frac{B \lambda_{g}}{2 \lambda_{g 0}} \sin (\psi)
\end{array}\right]
\end{aligned}
$$

By equating Equation 4.33 to an inverter with the transfer matrix given below

$$
\left[\begin{array}{cc}
0 & -\frac{j \lambda_{g}}{K \lambda_{g 0}} \\
-\frac{j K \lambda_{g}}{\lambda_{g 0}} & 0
\end{array}\right]
$$

Where $\mathrm{K}$ is the characteristic admittance when $\lambda_{g}=\lambda_{g 0}$. Hence

$$
\cos \left(\psi_{0}\right)+\frac{B}{2} \sin \left(\psi_{0}\right)=0
$$


That is

$$
\psi_{0}=-\tan ^{-1}\left(\frac{2}{B}\right)
$$

Now subtracting the $C_{A B C D}$ parameter from the $B_{A B C D}$ parameter in each matrix and equating at $\lambda_{g 0}$, we obtain

$$
K-\frac{1}{K}=B\left[\sin ^{2}\left(\frac{\psi}{2}\right)+\cos ^{2}\left(\frac{\psi}{2}\right)\right]=B
$$

The susceptance B of the inductive iris is positive. Therefore

$$
\frac{1}{K}<K \text { And } K>1
$$

From Equation 4.36 with B positive, $\psi_{0}$ must be negative, therefore, the line cannot be realized in isolation. In practice, impedance inverter is connected to lengths of waveguides and negative lengths are absorbed in adjacent waveguide lengths. Therefore, a shunt inductive iris can be represented by an inverter with reference planes defined by Equation 4.36 and the physical reference planes as shown in Figure 4.6. Since the design equations are strictly valid at $\lambda_{g 0}$, therefore, it is useful to check the iris approximation over broad bandwidth. Examining the $A_{A B C D}$ parameter in Equation 4.33

$$
\cos \left(\psi_{0} \frac{\lambda_{g 0}}{\lambda_{g}}\right)+\frac{B \lambda_{g}}{2 \lambda_{g 0}}+\sin \left(\psi_{0} \frac{\lambda_{g 0}}{\lambda_{g}}\right)
$$

And from Equation 4.36

$$
\frac{\mathrm{B}}{2}=-\frac{1}{\tan \left(\psi_{0}\right)}
$$

Hence

$$
\mathrm{A}_{\mathrm{ABCD}}=\cos \left(\psi_{0} \frac{\lambda_{g 0}}{\lambda_{g}}\right)-\frac{\lambda_{g}}{\lambda_{g 0} \tan \left(\psi_{0}\right)} \sin \left(\psi_{0} \frac{\lambda_{g 0}}{\lambda_{g}}\right)
$$

Differentiating $\mathrm{A}_{\mathrm{ABCD}}$ with respect to $\lambda_{g}$, we obtain

$$
\begin{aligned}
\frac{\mathrm{dA}_{\mathrm{ABCD}}}{d \lambda_{g}}= & \psi_{0} \frac{\lambda_{g 0}}{\lambda_{g}^{2}} \sin \left(\psi_{0} \frac{\lambda_{g 0}}{\lambda_{g}}\right)-\frac{\lambda_{g}}{\lambda_{g 0} \tan \left(\psi_{0}\right)} \sin \left(\psi_{0} \frac{\lambda_{g 0}}{\lambda_{g}}\right) \\
& +\frac{\lambda_{g}}{\lambda_{g 0} \tan \left(\psi_{0}\right)} \cos \left(\psi_{0} \frac{\lambda_{g 0}}{\lambda_{g}}\right)\left(\psi_{0} \frac{\lambda_{g 0}}{\lambda_{g}^{2}}\right)
\end{aligned}
$$


And if $\psi_{0}$ is relatively small

$$
\frac{\mathrm{dA}_{\mathrm{ABCD}}}{d \lambda_{g}} \approx \frac{\psi^{2} \lambda_{g 0}^{2}}{\lambda_{g}}-\frac{1}{\lambda_{g}}+\frac{1}{\lambda_{g}}=\frac{\psi^{2} \lambda_{g 0}^{2}}{\lambda_{g}}
$$

Which is small for $\psi_{0}$ small: Hence $\mathrm{A}$ is approximately zero over a relatively broad bandwidth. Furthermore, examining the B parameter in Equation 4.33 we obtain

$$
B_{A B C D}=\sin \left(\psi_{0} \frac{\lambda_{g 0}}{\lambda_{g}}\right)-\frac{2 \lambda_{g}}{\tan \left(\psi_{0}\right) \lambda_{g 0}} \sin ^{2}\left(\frac{\psi_{0}}{2} \frac{\lambda_{g 0}}{\lambda_{g}}\right)
$$

Again differentiating $\mathrm{A}_{\mathrm{ABCD}}$ with respect to $\lambda_{g}$ we obtain

$$
\begin{aligned}
\frac{d B_{A B C D}}{d \lambda_{g}} & =-\psi_{0} \frac{\lambda_{g 0}}{\lambda_{g}^{2}} \cos \left(\psi_{0} \frac{\lambda_{g 0}}{\lambda_{g}}\right)-\frac{2}{\tan \left(\psi_{0}\right) \lambda_{g 0}} \sin ^{2}\left(\frac{\psi_{0}}{2} \frac{\lambda_{g 0}}{\lambda_{g}}\right) \\
& +\frac{4 \lambda_{g}}{\tan \left(\psi_{0}\right) \lambda_{g 0}} \sin \left(\frac{\psi_{0}}{2} \frac{\lambda_{g 0}}{\lambda_{g}}\right) \cos \left(\frac{\psi_{0}}{2} \frac{\lambda_{g 0}}{\lambda_{g}}\right) \frac{\psi_{0}}{2} \frac{\lambda_{g 0}}{\lambda_{g}^{2}}
\end{aligned}
$$

And for small $\psi_{0}$

$$
\frac{d B_{A B C D}}{d \lambda_{g}}=-\frac{\psi_{0}}{2} \frac{\lambda_{g 0}}{\lambda_{g}^{2}}
$$

Now the differential of the $\mathrm{B}_{\mathrm{ABCD}}$ parameter in Equation 4.34 is given by

$$
\frac{d B}{d \lambda_{g}}=\frac{\lambda_{g 0}}{K \lambda_{g}^{2}}
$$

Since $\psi_{0}$ is negative, the differential of two $\mathrm{B}_{\mathrm{ABCD}}$ parameters will have the same functional behavior with respect to $\lambda_{g}$. Therefore, the inductive iris embedded in a waveguide section is a good approximation to an inverter over broad bandwidths.

A HFSS ${ }^{\mathrm{TM}}$ simulation of loosely coupled ceramic waveguide is carried out to model the different coupling bandwidths between ceramic waveguide resonators. The amount of coupling between two resonator sections of a ceramic waveguide can be controlled by symmetrically placed metal coated through holes in the broad dimension of the waveguide. Figure 4.6 shows the inter resonator coupling arrangement of two 1842.5 $\mathrm{MHz}$ ceramic waveguide resonators achieved by two symmetrically placed through holes in between them. The graph shown in Figure 4.9 is achieved with the hole diameter of $d_{h}=1.72 \mathrm{~mm}$ and an offset of $x_{1} \mathrm{~mm}$ from side walls of the waveguide. 
The percentage fractional bandwidth $\left(\frac{B W}{f_{0}} \times 100\right)$ achieved is plotted against the different percentage fractional hole offset $\left(\frac{x_{1}}{a} \mathrm{x} 100\right)$ from side walls.

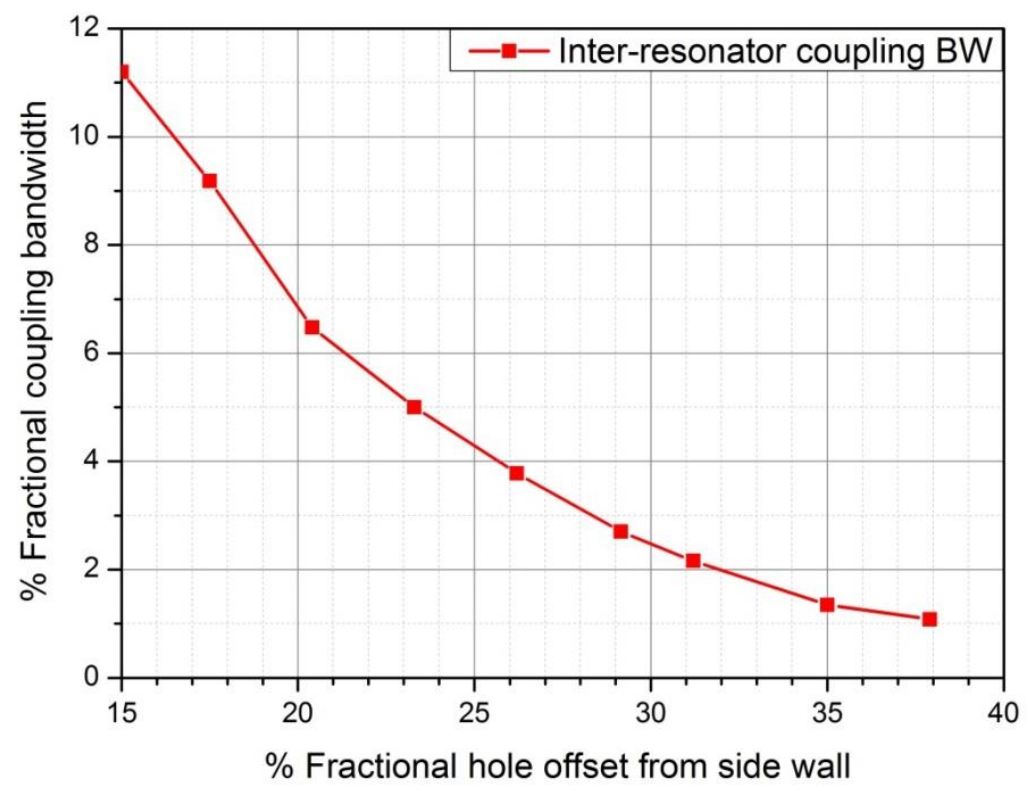

Figure 4.9: \% Fractional coupling bandwidth with different hole distance from sidewall

It is observed that by keeping the diameter of the through holes fixed and increasing the offset from side walls decreases the coupling bandwidth between the resonators. The data shown in Figure 4.9 can be interpolated to obtain the required coupling bandwidth extracted from the coupling matrix of the filter. The metal coated through holes are placed symmetrically to suppress the higher order modes. The required coupling bandwidths can be calculated first mathematically and then the position of holes can be adjusted for each individual inter resonator coupling.

\subsection{External coupling}

For achieving external couplings in a rectangular ceramic waveguide filter, a coaxial probe can be placed at the high E-field region of the first and last resonator of the filter. The inner conductor of coaxial probe acts as an antenna to excite the energy inside the ceramic waveguide. Electric field has its maximum value at the centre of the broad wall of rectangular ceramic waveguide. Therefore, a probe placed at this position excites strong E-field inside the waveguide. Four variables probe position, its distance from the 
shorted back end, depth inside the waveguide and the diameter of the probe determine its frequency, bandwidth and power handling capability [59]. The characteristic impedance of a coaxial cable can be calculated from the relation,

$$
Z_{C}=\frac{1}{2 \pi} \sqrt{\frac{\mu_{0} \mu_{r}}{\varepsilon_{0} \varepsilon_{r}}} \ln \left(\frac{D_{c}}{d_{c}}\right)
$$

Where ' $\mathrm{d}_{\mathrm{c}}$ ' and ' $\mathrm{D}_{\mathrm{c}}$ ' denote the inner and outer conductor diameter, respectively. The ratio $D_{c} / d_{c}=2.3$ gives $50 \Omega$ impedance of the air-filled coaxial cable. Rectangular waveguide impedance for TE mode can be calculated as [50]

$$
Z_{w}=\frac{2 b j \omega \mu}{a \gamma}
$$

Where $a$ and $b$ represent broad and narrow dimension of the waveguide respectively. Usually, air-filled waveguide impedance is much greater than the coaxial probes but in ceramic waveguide this mismatch ratio becomes much smaller due to dielectric loading of the waveguide. The external Q-factor for the coaxial probe excited cavity can be computed as [59]

$$
Q_{e}=\frac{0.5 \pi\left(\frac{\lambda_{g 0}}{\lambda_{0}}\right)^{2}}{\left(\frac{K_{01}}{\sqrt{Z_{w} Z_{c}}}\right)^{2}}
$$

Where ' $Z_{\mathrm{w}}$ ' and ' $\mathrm{Z}_{\mathrm{c}}$ ' represent waveguide and coaxial probe impedance respectively [59]. For filtering operation the probe excited cavity is usually loosely coupled resonator and the probe depth inside the waveguide is always much smaller than the operating wavelength. Probe diameter, its depth inside the waveguide and its distance from short circuited end are the input variables to fine tune the coaxial to waveguide input and output couplings [59]. Dielectric waveguide filters can be excited directly to first and last resonators to couple energy in and out. Usually a best guess is to place the probe at a distance of $\lambda_{g} / 4$ away from short circuited ends and then tune the depth of the probe inside the waveguide to get best return loss [60]. Adding another dielectric material around the probe concentrates the E-field near the surface of the probe thus increases coupling. Due to symmetric nature of the filter the input and the output probes should be at the same distance from the short circuited ends. Usually, a separate coaxial 
to waveguide transitional cavity is used to excite waveguide but this will increase overall volume of the filter. To achieve further miniaturization, it is preferred to insert input/output probes directly to the first and last resonator sections.

\subsection{Ceramic waveguide filter design process}

To design a rectangular dielectric waveguide filter, first step of implementation is to calculate suitable dimensions for ceramic waveguide resonator. As calculated earlier a resonator $\left(\varepsilon_{r}=45\right)$ with dimensions $17.15 \times 17.15 \times 8.60(\mathrm{~mm})$ resonates at a centre frequency of $1.8425 \mathrm{GHz}$. The effective wavelength of the wave in the ceramic material with a dielectric constant of 45 at a frequency of $1842 \mathrm{MHz}$ becomes,

$$
\begin{gathered}
\lambda_{d}=\frac{\lambda_{0}}{\sqrt{\varepsilon_{r}}} \\
=\frac{0.1628}{\sqrt{45}}=24.30 \mathrm{~mm}
\end{gathered}
$$

From Table 4-1, the specified centre frequency of bandpass filter is $1842.5 \mathrm{MHz}$ to be used for GSM-1800 downlink. Therefore the lower and upper effective guide wavelengths can be computed as [3],

$$
\lambda_{g 0 n}=\frac{\lambda_{d 0 n}}{\left[1-\left(\frac{\omega_{c}}{\omega_{n}}\right)^{2}\right]^{\frac{1}{2}}}
$$

This gives

$\lambda_{g 01}=35.8 \mathrm{~mm}$

$\lambda_{g 02}=33 \mathrm{~mm}$

Where $\lambda_{d 01}$ and $\lambda_{d 02}$ are the lower and upper pass band edge wavelengths in dielectric medium. And the effective guide wavelength of the filter is expressed as

$$
\lambda_{g 0}=\frac{\lambda_{g 01}+\lambda_{g 02}}{2}+\frac{\left[\lambda_{g 01} * \cos \left(\frac{\pi \lambda_{g 02}}{2 \lambda_{g 01}}\right)+\lambda_{g 02} * \cos \left(\frac{\pi \lambda_{g 01}}{2 \lambda_{g 02}}\right)\right]}{\left[\pi \sin \left(\pi \frac{\lambda_{g 02}}{\lambda_{g 01}}\right)+\pi \sin \left(\pi \frac{\lambda_{g 01}}{\lambda_{g 02}}\right)\right]}=34.4 \mathrm{~mm}
$$

Where $\alpha$ is defined as 


$$
\begin{gathered}
\alpha=\left[\frac{\lambda_{g 01}}{\lambda_{g 0}} \sin \left(\pi \frac{\lambda_{g 01}}{\lambda_{g 0}}\right)\right]^{-1} \\
=7.82
\end{gathered}
$$

As a discontinuity in a waveguide is equivalent to the impedance inverter in a cascaded unit element circuit, therefore, a waveguide filter can be approximated as a cascade of unit elements with inverters. The Chebyshev bandpass response for a waveguide bandpass filter is given as

$$
\left|S_{12}(j \omega)\right|^{2}=\frac{1}{1+\varepsilon^{2} T_{N}^{2}\left[\alpha\left(\frac{\lambda_{g}}{\lambda_{g 0}}\right) \sin \left(\frac{\pi \lambda_{g 0}}{\lambda_{g}}\right)\right.}
$$

Then order of the waveguide bandpass filter is determined according to the maximum allowed passband insertion loss and stop band rejection specifications. The insertion loss function for a Chebyshev waveguide filter is

$$
I L=10 \log \left[1+\varepsilon^{2} T_{N}^{2}\left[\alpha\left(\frac{\lambda_{g}}{\lambda_{g 0}}\right) \sin \left(\frac{\pi \lambda_{g 0}}{\lambda_{g}}\right)\right]\right]
$$

Normalised impedance of each resonator (Normalised to unity where $Z o=Z n+1=$ $1 \Omega)$ can be calculated as [3]

$$
Z_{r}=\frac{2 \alpha \sin }{\eta}\left[\frac{(2 r-1) \pi}{2 N}\right]-\frac{\frac{1}{4 \eta \alpha}\left[\eta^{2}+\sin ^{2}\left(\frac{r \pi}{N}\right)\right]}{\sin \left[\frac{(2 r+1) \pi}{2 N}\right]}-\frac{\frac{1}{4 \eta \alpha}\left[\eta^{2}+\sin ^{2}\left(\frac{r-1) \pi}{N}\right)\right]}{\sin \left[\frac{(2 r-3) \pi}{2 N}\right]}
$$

Where

$$
\begin{aligned}
\eta & =\sinh \left[\frac{1}{N} \sinh ^{-1}\left(\frac{1}{\epsilon}\right)\right] \\
& =0.52
\end{aligned}
$$

This gives,

$$
\begin{aligned}
& Z_{0}=Z_{7}=1 \\
& Z_{1}=Z_{6}=7.772 \\
& Z_{2}=Z_{5}=21.23
\end{aligned}
$$


$Z_{3}=Z_{4}=29$

The coupling coefficients for inter resonator inductive posts for a Chebyshev filter are calculated as

$$
K_{r, r+1}=\frac{\left[\eta^{2}+\sin ^{2}\left(\frac{r \pi}{N}\right)\right]^{\frac{1}{2}}}{\eta}
$$

This gives

$K_{\text {in } 1}=K_{6 o u t}=1$

$K_{12}=K_{56}=1.38$

$K_{23}=K_{45}=1.942$

$K_{34}=2.167$

The normalised susceptances of the inductive holes between the half wavelength ceramic waveguide resonators can be approximated as

$$
B_{r, r+1}=\frac{\left(Z_{r} Z_{r+1}\right)^{\frac{1}{2}}}{K_{r, r+1}}-\frac{K_{r, r+1}}{\left(Z_{r} Z_{r+1}\right)^{\frac{1}{2}}}
$$

This gives,

$B_{\text {in } 1}=B_{6 o u t}=2.45$

$B_{12}=B_{56}=9.34$

$B_{23}=B_{45}=12.96$

$B_{34}=13.58$

Actual phase lengths of waveguides between the metal coated holes in a ceramic waveguide can be calculated by subtracting the negative lengths of the guide from a half wavelength, giving

$$
\varphi_{r}=\pi-\frac{1}{2}\left[\cot ^{-1}\left(\frac{B_{r-1, r}}{2}\right)+\cot ^{-1}\left(\frac{B_{r, r+1}}{2}\right)\right]
$$

This gives, 
$\varphi_{1}=\varphi_{6}=2.69$

$\varphi_{2}=\varphi_{5}=2.95$

$\varphi_{3}=\varphi_{4}=2.99$

Phase lengths calculated by Equation 4.61 are in radians, thus, actual physical lengths of resonators between posts centres can be calculated as [49]

$$
d_{r}=\frac{\varphi_{r} \lambda_{g 0}}{2 \pi}
$$

This gives

$d_{1}=d_{6}=14.7 \mathrm{~mm}$

$d_{2}=d_{5}=16.2 \mathrm{~mm}$

$d_{3}=d_{4}=16.4 \mathrm{~mm}$

Thus the final transmission line model of a waveguide bandpass filter can be represented with resonators separated by inductive susceptances as shown in Figure 4.10.

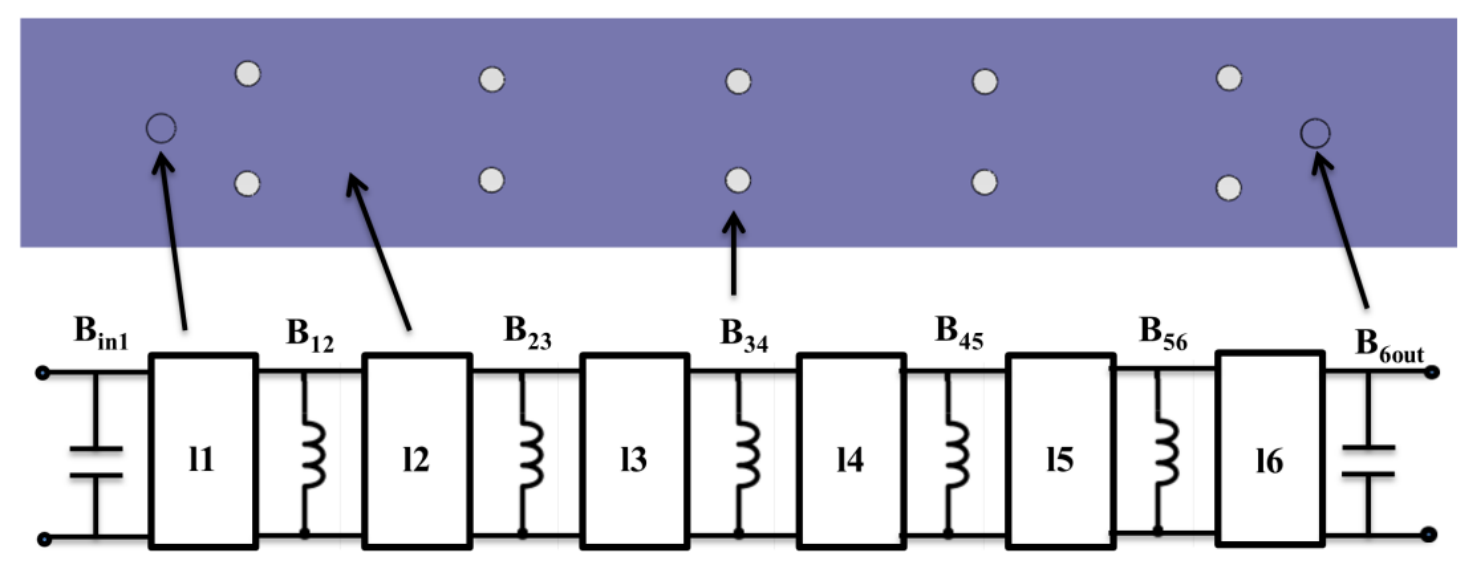

Figure 4.10: An equivalent circuit of six pole rectangular ceramic WG filter

\subsection{Results and measurements}

An integrated Chebyshev ceramic waveguide filter is designed and fabricated. Table 4-3 summarises the physical dimensions of the fabricated filer. Figure 4.12 shows the 
comparison between EM simulation and fabricated filter measured results. Measured results are in good agreement with the simulated results with the one exception of high passband insertion loss. Measured filter pass band insertion loss is increased mainly due to leakage at input and output terminals. These measurements on initial design were taken with the input and output pin not properly soldered in the blind hole in the ceramic. Therefore, leakage due to air gap between input/output pin and ceramic resonator increased the pass band loss of the filter. All future designs will include a test fixture to avoid any gaps between input/output pins and ceramic resonators so as to minimise leakage at these terminals. The bandwidth of measured filter is slightly narrower near upper pass band edge, due to insufficient coupling at input/output terminals. Although without any tuning screw in the design, the return loss of the measured filter is quite good but all five peaks of return loss can be recovered and could be improved to $20 \mathrm{~dB}$ if tuning screws are used to tune the resonant frequencies of individual resonators.

Table 4-3: Design filter dimensions obtained through $\operatorname{HFSS}^{\mathrm{TM}}$ simulation for fabrication

\begin{tabular}{|l|l|l|}
\hline Dimension & Variable & Value \\
\hline Waveguide width & $a$ & $17.15 \mathrm{~mm}$ \\
\hline Waveguide height & $b$ & $8.6 \mathrm{~mm}$ \\
\hline Resonator 1 and 6 length & $d_{1}=d_{6}$ & $15.97 \mathrm{~mm}$ \\
\hline Resonator 2 and 5 length & $d_{2}=d_{5}$ & $16.86 \mathrm{~mm}$ \\
\hline Resonator 3 and 4 length & $d_{3}=d_{4}$ & $17.02 \mathrm{~mm}$ \\
\hline Diameter of all through holes & $d_{h}$ & $0.86 \mathrm{~mm}$ \\
\hline Through hole distance from side walls for $K_{12} \& K_{56}$ & $x_{1}$ & $4.77 \mathrm{~mm}$ \\
\hline Through hole distance from side walls for $K_{23} \& K_{45}$ & $x_{2}$ & $5.1 \mathrm{~mm}$ \\
\hline Through hole distance from side walls for $K_{34}$ & $x_{3}$ & $5.16 \mathrm{~mm}$ \\
\hline Input/output probe radius & $r_{p}$ & $1 \mathrm{~mm}$ \\
\hline Input/output probe depth inside waveguide & $h$ & $4.07 \mathrm{~mm}$ \\
\hline I/O probe distance from shorted back end & $d_{y}$ & $10 \mathrm{~mm}$ \\
\hline
\end{tabular}


Figure 4.11 shows the photograph of the fabricated integrated ceramic waveguide Chebyshev filter.

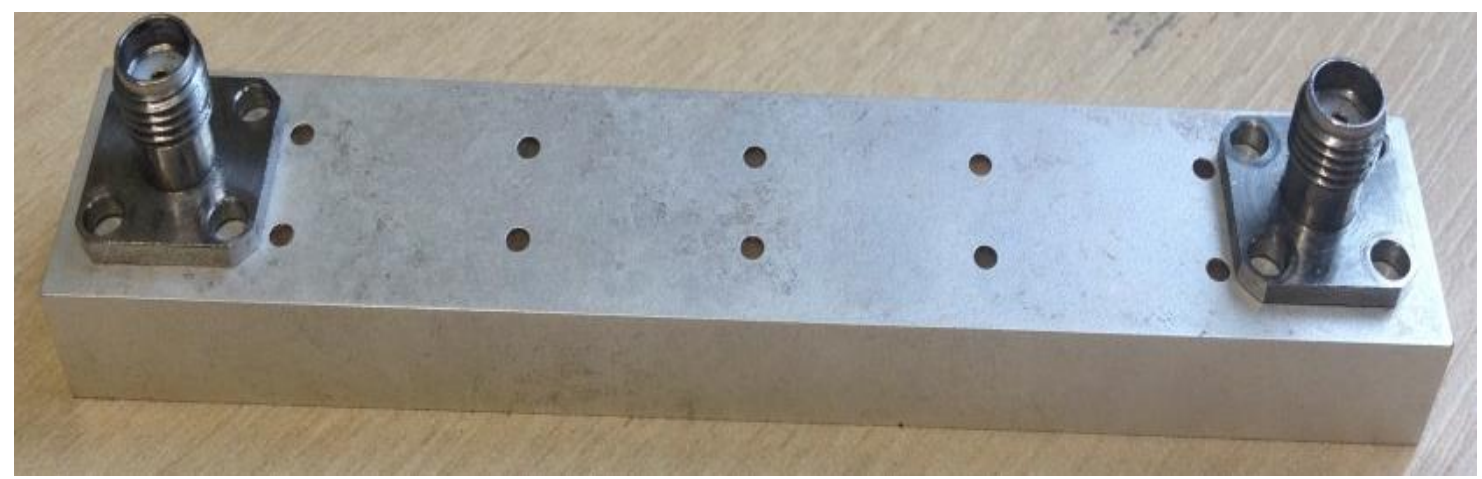

Figure 4.11: Fabricated six pole Chebyshev ceramic Waveguide filter

A comparison of simulated and measured results is given in Figure 4.12. Figure 4.13 shows the detailed pass band insertion loss of simulated and measured filter.

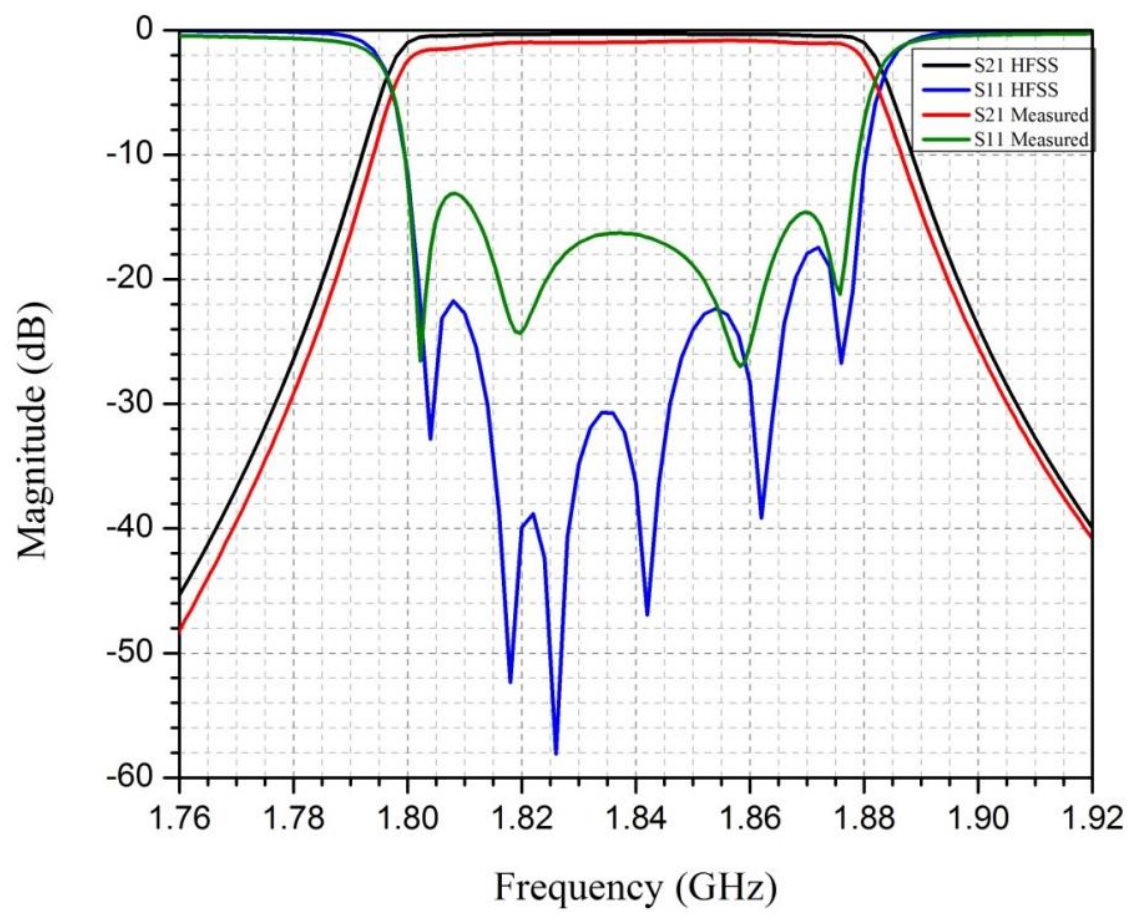

Figure 4.12: $1842.5 \mathrm{MHz}$ ceramic Waveguide band pass filter measured \& simulated response 


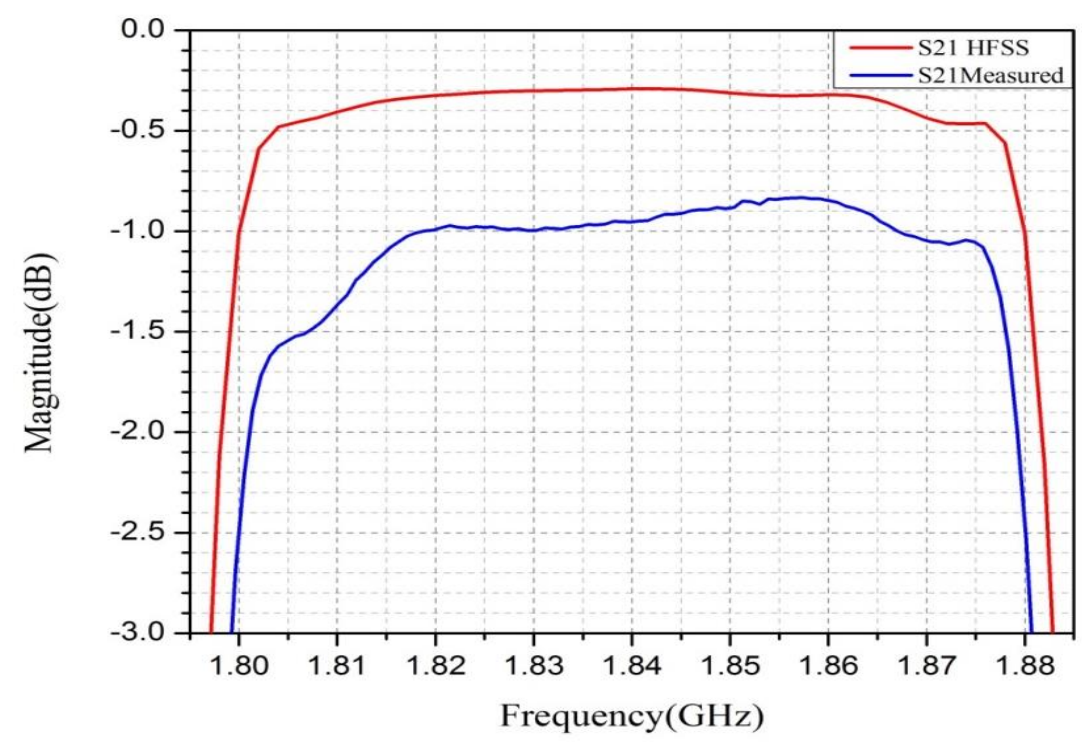

Figure 4.13: $1842 \mathrm{MHz}$ ceramic Waveguide filter passband insertion loss (measured vs HFSS $^{\mathrm{TM}}$ )

The degraded performance of fabricated filter is mainly due to leakage at input and output terminals. A careful soldering of input/output pins to ceramic waveguide can bring measurements well in agreement to the simulated response.

\subsection{Summary}

An integrated ceramic waveguide filter is designed and fabricated. It provides significant volume reduction in contrast to conventional waveguide or air-filled coaxial filters used in cellular base stations. The design is based on low pass prototype of ladder network. Impedance and frequency scaling is done using insertion loss method of filter design. Input and output coupling are achieved by coaxial pins placed at the centre of the broad wall of the first and last waveguide resonator cavity. Inter resonator couplings are achieved using metal coated through holes. The structure is silver coated except input/output probe positions to avoid any radiation loss. The measured results are in good agreement with EM simulation results except from pass band insertion loss which is mainly due to leakage at input and output. 


\section{GENERALIZED CHEBYSHEV}

\section{CERAMIC RECTANGULAR WAVEGUIDE BANDPASS FILTER}

\subsection{Introduction}

Transmit and receive filter banks used in a cellular diplexer share common antenna port and require high attenuation in opposite bands. In some cases guard band between transmit and receive filter frequencies is very small, therefore, asymmetric filter response with high rejection is required. The out of band rejection of a filter is directly proportional to the number of elements in the filter but at the same time adding more resonators in a filter, directly increases pass band insertion loss and overall volume, mass and eventually cost of the filter. Alternatively, to achieve higher out of band rejection with less number of elements in the filter is to use well-known technique of cross couplings between non-adjacent resonators. Cross coupling can be used to introduce a finite frequency real or imaginary transmission zero. A finite frequency transmission zero is produced due to the destructive interference of multipath (direct and cross coupled) in a cross coupled arrangement [21]. Kurzrok [61, 62] introduced cross coupled triplet and quadruplet in waveguide band pass filter in the early stages of microwave filter designs. The cross coupled path could carry energy either by capacitive or inductive coupling. The sign of the coupling determines the position of the transmission zero at either below or above the passband. And the amount of coupling allowed through the cross coupled path determines the relative position of the 
transmission zero from the pass band. Usually a weaker cross coupling is used so as to place the transmission zero not too close to the pass band, in which case it can degrade the return loss in the passband of the filter. The most common forms of cross coupling configuration are triplet or quadruplet.

A generalized Chebyshev ceramic waveguide filter with transmission zeros at below and above the pass band of the filter is described in this Chapter to meet stringent out of band rejection specifications. Both inductive and capacitive waveguide triplets are introduced to realize positive and negative cross couplings. There are several methods in the literature to introduce transmission zeros in a waveguide band pass filter e.g. by cross couplings between non-adjacent resonators [5, 63] or by introducing suitable parasitic resonators in the filter $[22,64,65]$. Different blind and through holes are used to realize the cross coupling triplets in the ceramic waveguide filter. These coupling structures are discussed later in the Chapter.

\subsection{Filter specifications and design}

A generalized Chebyshev ceramic rectangular waveguide filter with following passband and stopband specification is designed in this Chapter.

Table 5-1: Genrealised Chebyshev bandpass filter specifications

\begin{tabular}{|l|l|}
\hline Centre frequency & $1730 \mathrm{MHz}$ \\
\hline Passband Bandwidth & $60 \mathrm{MHz}$ \\
\hline Passband Insertion loss & $\leq 0.5 \mathrm{~dB}$ \\
\hline Order of the filter & 6 \\
\hline Passband Return loss & $\geq 20 \mathrm{~dB}$ \\
\hline Stopband attenuation & $\begin{array}{l}\geq 70 \mathrm{~dB} \text { at } \mathrm{DC}<\mathrm{f}<1650 \mathrm{MHz} \\
\geq 90 \mathrm{~dB} \text { at } 1880 \mathrm{MHz}<\mathrm{f}<2000 \mathrm{MHz}\end{array}$ \\
\hline
\end{tabular}

The generalised Chebyshev approximation offers equiripple response in the passband of the filter with arbitrarily placed transmission zeros in the stopband of the filter. A generalised Chebyshev filter transfer function approximation is given by 


$$
\left|S_{12}(j \omega)\right|^{2}=\frac{1}{1+\varepsilon^{2} F_{N}^{2}(\omega)}
$$

With

$$
-1<F_{N}(\omega)<+1 \text { For }-1<\omega<+1
$$

And

$$
F_{N}\left(\omega_{r}\right)=\infty \text { for } r=1,2,3 \ldots . N
$$

The attenuation poles produced in the stopband of the filter can be generated either by allowing cross couplings between non-adjacent resonators or by extracted pole techniques.

\subsubsection{Order of the filter}

The order of the filter can be calculated from the relation [66]

$$
I L(d B)=\frac{8.6[N-1.5] f_{0}}{B w Q}
$$

Where $\mathrm{N}$ is the order of the filter, $\mathrm{Bw}$ is the passband bandwidth and $\mathrm{f}_{0}$ is the centre frequency of the filter. For maximum $I L=0.5 \mathrm{~dB}, f_{0}=1730 \mathrm{MHz}, B \mathrm{w}=60 \mathrm{MHz}$ and unloaded $Q=2500$, the order of the filter is restricted to 6 .

$$
\begin{gathered}
N \leq \frac{I L * B w * Q}{8.6 f_{0}}+1.5 \\
N \leq 6.5
\end{gathered}
$$

\subsubsection{Coupling matrix for the filter}

A microwave filter can be defined by the Q-factor of its resonators and inter resonator and external coupling bandwidths of the filter. The concept of coupling bandwidths first used by Dishal [67, 68], greatly simplifies the design procedure of the microwave filters. Coupling bandwidth is an alternative way to represent the impedance or admittance inverters. Normalized coupling bandwidths are calculated from the element values of a low pass prototype filter network. The external normalised couplings coefficients of a normalised filter can be expressed as

$$
K_{01}=\frac{1}{g_{0} g_{1}}
$$


And

$$
q_{\text {Nout }}=\frac{1}{g_{N} g_{N+1}}
$$

And the inter-resonator normalised coupling coefficients can be calculated as

$$
k_{i j}=\frac{1}{\sqrt{g_{i} g_{i+1}}}
$$

It is sometime more convenient to represent coupling coefficients into normalised coupling bandwidths. The filter passband bandwidth is multiplied to coupling coefficients to convert them into coupling bandwidths. The coupling matrix for the generalized Chebyshev filter was derived with the method described in [22] and is given in Figure 5.1.

$$
\left[\begin{array}{ccccccccc} 
& S & 1 & 2 & 3 & 4 & 5 & 6 & L \\
S & 0 & 1.0626 & 0 & 0 & 0 & 0 & 0 & 0 \\
1 & 1.0626 & -0.0035 & 0.8897 & 0.1218 & 0 & 0 & 0 & 0 \\
2 & 0 & 0.8897 & -0.1749 & 0.6232 & 0 & 0 & 0 & 0 \\
3 & 0 & 0.1218 & 0.6232 & 0.0101 & 0.5993 & 0 & 0 & 0 \\
4 & 0 & 0 & 0 & 0.5993 & -0.0341 & 0.6097 & -0.1969 & 0 \\
5 & 0 & 0 & 0 & 0 & 0.6097 & 0.2713 & 0.8761 & 0 \\
6 & 0 & 0 & 0 & 0 & -0.1969 & 0.8761 & -0.0035 & 1.0626 \\
L & 0 & 0 & 0 & 0 & 0 & 0 & 1.0626 & 0
\end{array}\right]_{\text {? }}
$$

Figure 5.1: Coupling matrix of generalised Chebyshev bandpass filter

A lumped element six section generalized Chebyshev bandpass filter based on above copling matrix is represented in the Figure 5.3. The bandwidth of the filter to be designed is $0.06 \mathrm{GHz}$, Therefore, the coupling coefficients in terms of coupling bandwidths using Equation 4.11 become,

$K_{e}=0.0638 \mathrm{GHz}$

$K_{12}=0.0533 \mathrm{GHz}$ 


$$
\begin{aligned}
& K_{23}=0.0374 \mathrm{GHz} \\
& K_{34}=0.0359 \mathrm{GHz} \\
& K_{45}=0.0365 \mathrm{GHz} \\
& K_{56}=0.0526 \mathrm{GHz} \\
& K_{13}=0.0070 \mathrm{GHz} \\
& K_{46}=-0.011 \mathrm{GHz}
\end{aligned}
$$

\subsubsection{Lumped element impedance inverters}

The lumped element bandpass filter can be designed by using impedance inverter coupled shunt or series resonators. The inductor used to realize external coupling impedance inverter of the bandpass filter as shown in Figure 5.2 can be calculated using Equation 4.12 as

$$
L_{e}=17.236 n H
$$

And the inductor used to form an impedance inverter between adjacent resonators of the bandpass filter as shown in Figure 5.2 can be obtained using Equation 4.13. This gives,

$$
\begin{aligned}
& L_{12}=95.046 n H \\
& L_{23}=135.454 n H \\
& L_{34}=141.114 n H \\
& k_{45}=138.794 n H \\
& L_{56}=96.311 n H \\
& L_{13}=723.714 n H
\end{aligned}
$$

The negative impedance inverter can be realized as shown in Figure 5.2(b) and the capacitor value can be calculated as [57]

$$
C_{i j}=\frac{K_{i j(G H z)}}{4 f^{2} Z_{0}} n F
$$


For $Z_{0}=50$ and $K_{46(G H z)}=0.011$ at centre frequency of the filter,

$$
C_{46}=0.0183 p F
$$

Figure 5.2 shows the impedance inverter block diagram and its equivalent lumped element circuit diagram.

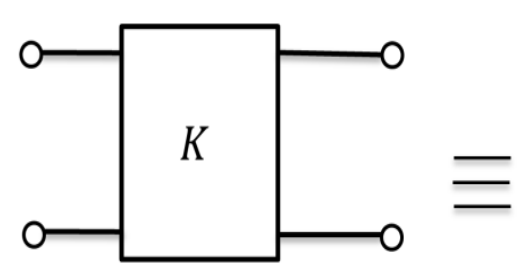

(a)

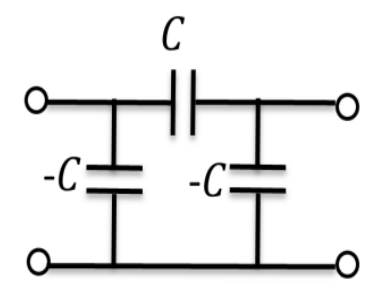

(b)

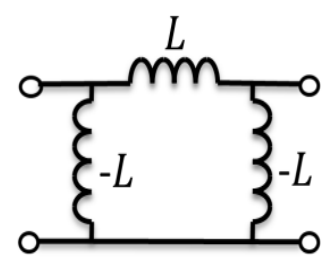

(c)

Figure 5.2: K-inverter and its equivalent lumped element circuit

The element values of shunt resonator with resonance $f_{0 R X}$ and system impedance level of $Z_{0}=50 \Omega$ can be calculated as from [7]

$$
C=\frac{1}{4 f_{0 R X(G H z)} Z_{0}} n F \quad r=1,2 . .6
$$

And

$$
L=\frac{Z_{0}}{\pi^{2} f_{0 R X(G H z)}} n H \quad r=1,2 . .6
$$

Where deviation of $f_{0 R X}$ from centre frequency of the filter can be calculated directly from the self-coupling coefficients of the coupling matrix. Figure 5.3 shows the layout of the lumped element inverter coupled bandpass filter with two cross coupled triplets to realize two transmission zeros in its response. Figure 5.4 shows the lumped element response of the six section generalized Chebyshev bandpass filter. 


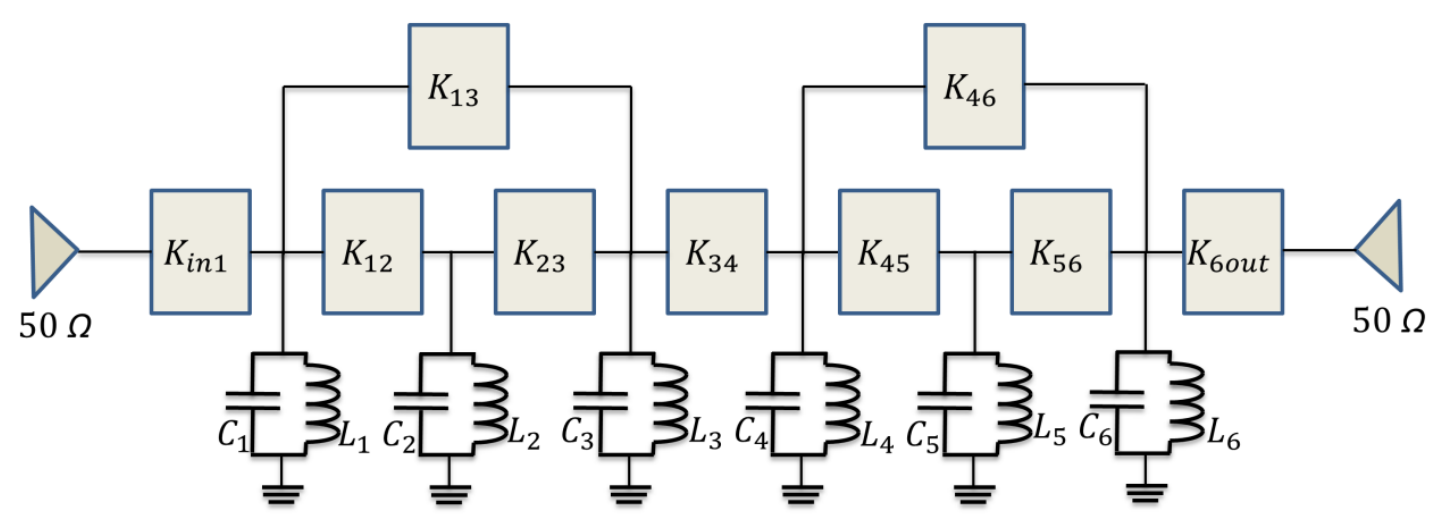

Figure 5.3: Inverter coupled generalized Chebyshev bandpass filter circuit design

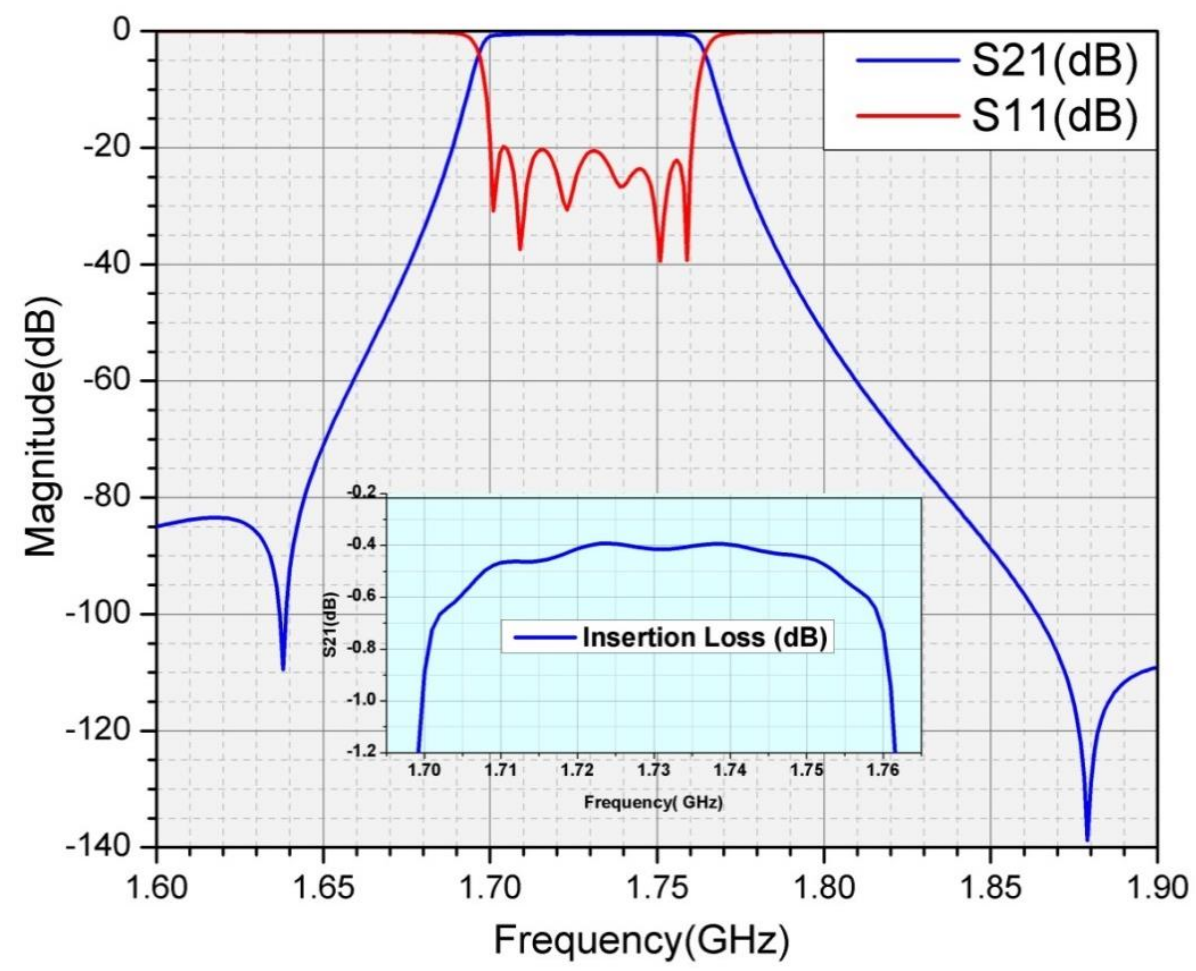

Figure 5.4: Simulated lumped element generalized Chebyshev bandpass filter response

An inductive cross coupled path is allowed from resonator 1 to resonator 3 to generate a transmission zero above the passband at a frequency of $1880 \mathrm{MHz}$. The coupling coefficent $K_{13}$ controls the relative position of transmissison zero above passband. Another cross coupled path is added with negative coupling coefficient to produce a transmisssion zero below the passband at a frequency of $1640 \mathrm{MHz}$ to meet the 
specified out of band attenuation criteria.

\subsection{Ceramic waveguide resonator design}

A ceramic waveguide resonator composed of barium-titanate $\left(\mathrm{BaTiO}_{3}\right)$ material with relative permittivity of 43 and dielectric loss tangent of 0.00004 is used. The EM simulations show a Q-factor of 2400 at a frequency of $1.730 \mathrm{GHz}$. The dimensions of the ceramic waveguide resonator are calculated based on the Equation 3.1 for fundamental mode $\left(\mathrm{TE}_{10}\right)$ resonance of ceramic waveguide resonator. For a rectangular ceramic waveguide resonator with an aspect ratio of $\frac{b}{a}=0.5$, by putting $a=d=2 b$, $f_{0}=1730 \mathrm{MHz}$ and $\varepsilon_{r}=43$ in Equation 3.1, we get

$$
a=d=2 b=18.64 \mathrm{~mm}
$$

Therefore the silver plated ceramic waveguide resonator with an aspect ratio of 0.5 has the dimensions of $18.64 \times 18.64 \times 9.32(\mathrm{~mm})$ to resonate at a fundamental frequency of $1730 \mathrm{MHz}$. The effective wavelength of the wave in the ceramic material with a dielectric constant of 43 at a frequency of $1730 \mathrm{MHz}$ becomes,

$$
\begin{gathered}
\lambda_{\text {effective }}=\frac{\lambda_{0}}{\sqrt{\varepsilon_{r}}} \\
=\frac{0.1734}{\sqrt{43}}=26.4 \mathrm{~mm}
\end{gathered}
$$

Where using Equation 4.17

$$
\lambda_{0}=\frac{3 \times 10^{8}}{1730 \times 10^{6}}=0.1734 \mathrm{~m}
$$

The cut-off wavelength of the ceramic waveguide resonator with $a=18.64 \mathrm{~mm}$ can be calculated using Equation 4.18 as

$$
\lambda_{c}=37.28 \mathrm{~mm}
$$

Therefore cut-off frequency of the rectangular ceramic waveguide with its broad dimension equals to $a=18.64 \mathrm{~mm}$ can be calculated using Equation 4.19 as

$$
\omega_{c}=7.710 \times 10^{9}
$$


Hence using Equation 4.20,

$$
f_{C}=1.227 \mathrm{GHz}
$$

The Q-factor for the $\mathrm{TE}_{10}$ mode of the silver coated rectangular ceramic waveguide resonator with $a=d=2 b=18.64 \mathrm{~mm}$ is calculated using Equation 4.21 and Equation 4.23

$$
Q_{U}=3000
$$

Therefore, calculated unloaded Q-factor of a rectangular ceramic waveguide resonator at a frequency of $1730 \mathrm{MHz}$ is 3000 . By including the effect of dielectric loss tangent of the material i.e. $\tan \delta=0.00004$, in Equation 4.24, the total unloaded Q-factor becomes

$$
Q_{\text {total }}=2679
$$

The HFSS ${ }^{\mathrm{TM}}$ simulated Q-factor value was 2430 which is mainly because a more practical value of silver conductivity i.e. $4.4 \times 10^{7} \Omega^{-1} \mathrm{~m}^{-1}$ is used in simulation. Table 5-2 shows the fundamental resonance frequency, first spurious resonance and the Qfactor values of the ceramic waveguide resonator obtained from HFSS $^{\mathrm{TM}}$ simulation. It is observed that the first spurious resonance occurs at $2740 \mathrm{MHz}$ thus providing a wide spurious free stopband bandwidth.

Table 5-2: Ceramic waveguide resonator eigenmode data $\left(\mathrm{HFSS}^{\mathrm{TM}}\right)$

\begin{tabular}{|c|c|c|}
\hline Mode Number & Frequency (MHz) & Q-Factor \\
\hline 01 & 1732 & 2430 \\
\hline 02 & 2740 & 2700 \\
\hline
\end{tabular}

The length $(d)$ of the each resonator used in the filter can be modified slightly according to its centre frequency, dictated by the self-coupling coefficient value given in the coupling matrix. Table 5-3 summarises the modified lengths of each resonator according to the self-coupling coefficient given in the coupling matrix. 
Table 5-3: Modified resonator lengths according to self-coupling values

\begin{tabular}{|c|c|c|c|}
\hline Resonator & Self-coupling coefficient & Loaded Resonance(MHz) & Resonator length $(d)$ \\
\hline 01 & -0.00012 & 1731 & $18.70 \mathrm{~mm}$ \\
\hline 02 & -0.00606 & 1738 & $18.54 \mathrm{~mm}$ \\
\hline 03 & 0.00035 & 1732 & $18.64 \mathrm{~mm}$ \\
\hline 04 & -0.00118 & 1734 & $18.60 \mathrm{~mm}$ \\
\hline 05 & 0.0094 & 1723 & $18.87 \mathrm{~mm}$ \\
\hline 06 & -0.00012 & 1734 & $18.60 \mathrm{~mm}$ \\
\hline
\end{tabular}

\subsection{Inter resonator coupling}

Inter resonator coupling in the ceramic waveguide filter can be achieved by introducing shunt discontinuities between half wavelength resonators. The shunt discontinuity can be introduced either by through or blind holes placed in the broad wall of the waveguide at a period of half wavelength. The sign of coupling determines the nature of metal plated hole in the ceramic waveguide. Following section explains the inductive and capacitive coupling configurations in the ceramic waveguide.

\subsubsection{Inductive coupling}

For a rectangular ceramic block, inter resonator inductive couplings are achieved by metal plated circular through holes separated by half wavelength sections as explained in Section 4.5. Diameter of hole and its offset from the side wall determine the amount of coupling achieved. These metal plated holes are placed symmetrically across the broad wall of the waveguide at half wavelength intervals.

\subsubsection{Capacitive coupling}

A blind hole at the middle of the broad wall of the ceramic waveguide acts as a shunt capacitor. Amount of coupling achieved is a function of diameter and depth of blind silver plated hole. A blind hole or capacitive discontinuity shown in Figure 5.5 can be modelled as a shunt capacitor between half guide wavelength resonators. 


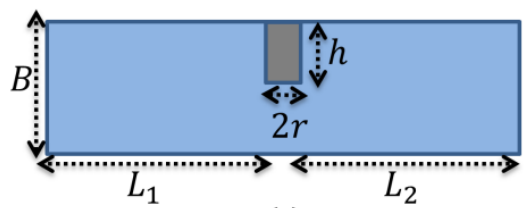

(i)

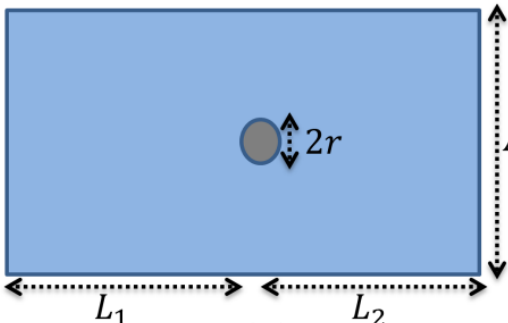

(ii)

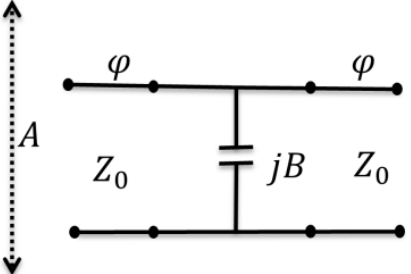

(iii)

Figure 5.5: Waveguide capacitive impedance inverter (i) Side view (ii) Top view (iii) Equivalent circuit

The transfer matrix of the capacitive shunt discontinuity embedded in a uniform length of waveguide with electrical length $\Psi$, can be written as

$$
\begin{gathered}
{[T]=\left[\begin{array}{cc}
\cos \Psi & j \sin \Psi \\
j \sin \Psi & \cos \Psi
\end{array}\right]\left[\begin{array}{cc}
1 & 0 \\
j B & 1
\end{array}\right]\left[\begin{array}{cc}
\cos \Psi & j \sin \Psi \\
j \sin \Psi & \cos \Psi
\end{array}\right]} \\
=\left[\begin{array}{cc}
\cos \Psi-B \sin \Psi & j \sin \Psi \\
j[\sin \Psi+B \cos \Psi] & \cos \Psi
\end{array}\right]\left[\begin{array}{cc}
\cos \Psi & j \sin \Psi \\
j \sin \Psi & \cos \Psi
\end{array}\right] \\
=\left[\begin{array}{cc}
\cos ^{2} \Psi-B \cos \Psi \sin \Psi-\sin ^{2} \Psi & j \sin \Psi[2 \cos \Psi-B \sin \Psi] \\
j \cos \Psi[2 \sin \Psi+B \cos \Psi] & \cos ^{2} \Psi-B \cos \Psi \sin \Psi-\sin ^{2} \Psi
\end{array}\right]
\end{gathered}
$$

In an ideal shunt capacitive impedance inverter transfer matrix $A_{A B C D}=D_{A B C D}=0$, therefore

$$
\begin{gathered}
\cos ^{2} \Psi-B \cos \Psi \sin \Psi-\sin ^{2} \Psi=0 \\
=>\cos ^{2} \Psi-\sin ^{2} \Psi=B \cos \Psi \sin \Psi \\
=>\quad \cos 2 \Psi=\frac{B \sin 2 \Psi}{2} \\
=>\quad \cos 2 \Psi=B \sin 2 \Psi \\
=>\quad B=2 \cot (2 \Psi)
\end{gathered}
$$

Also by comparing transfer matrix to an ideal shunt capacitor transfer matrix

$$
\begin{aligned}
& j K=j \cos \Psi[2 \sin \Psi+B \cos \Psi] \\
= & K=\cos \Psi[2 \sin \Psi+B \cos \Psi] \\
= & K=2 \cos \Psi \sin \Psi+B \cos ^{2} \Psi
\end{aligned}
$$


From trigonometric identity

$$
\cos ^{2} \Psi==[1+\cos (2 \Psi)] / 2 \text { and } 2 \cos \Psi \sin \Psi=\sin (2 \Psi)
$$

Therefore by putting in 5.16

$$
\begin{gathered}
K=\sin (2 \Psi)+B[1+\cos (2 \Psi)] / 2] \\
K=\sin (2 \Psi)+2 \cot (2 \Psi)[1+\cos (2 \Psi)] / 2] \\
K=\sin (2 \Psi)+\cot (2 \Psi)+\cot (2 \Psi) \cos (2 \Psi) \\
K=\sin (2 \Psi)+\cos (2 \Psi) / \sin (2 \Psi)+\cos ^{2}(2 \Psi) / \sin (2 \Psi) \\
k=[1+\cos (2 \Psi)] / \sin ((2 \Psi) \\
k=2 \cos ^{2}(\Psi) / 2 \sin (\Psi) \cos (\Psi) \\
k=\cot (\Psi)
\end{gathered}
$$

Now from eq:5.14

$$
B=2 \cot (2 \Psi)
$$

But

$$
\begin{gathered}
\cot (2 \Psi)=\left[1-\tan ^{2}(\Psi)\right] / 2 \tan \Psi \\
B=2\left[1-\tan ^{2}(\Psi)\right] / 2 \tan \Psi
\end{gathered}
$$

Put $\tan \Psi=1 / \cot \Psi=1 / k$, Thus

$$
\begin{aligned}
& B=\left[1-\frac{1}{k^{2}}\right] / 1 / k \\
& B=\left[k^{2}-1\right] / k \\
& \text { As }|\mathrm{k}|<1 \Rightarrow \mathrm{k}<0 \\
& =>B \text { is positive }
\end{aligned}
$$

From elementary circuit theory, capacitive susceptance can be written as

$$
j B=2 \pi \mathrm{fc}
$$

Or 


$$
c=\frac{B}{2 \pi f}
$$

Also from basic circuit theory, capacitance between two parallel plates of an area $\mathrm{A}_{\text {area }}$ and separation $d_{\text {separation }}$ between plates can be calculated as

$$
c=\frac{\varepsilon_{0} \varepsilon_{\mathrm{r}} A_{\text {area }}}{d_{\text {separation }}}
$$

Therefore dimensions of a blind hole in a ceramic waveguide acting as capacitive impedance inverter can be found from Equation 5.20 and Equation 5.23 for any particular value of ' $\mathrm{k}$ '.

\subsection{Cross coupled triplets}

In order to realize a generalised Chebyshev response, transmission zeros are needed to be produced in the stopband of the filter. There are several methods to achieve attenuation poles in the stop band of a waveguide filter. The most common and convenient way is to form a cross coupled triplet in the waveguide [61, 69]. The extra path added between non-adjacent resonators produce an attenuation pole in the stopband of the filter due to destructive interference of the signal coming from direct and indirect path. The sign of cross coupled path determines the position of the transmission zero either below or above the passband. The strong cross coupled paths produce attenuation poles near the passband but at the same time increase the group delay near the passband edge [21]. More than one transmission zero can be produced either below or above passband of the filter by adding multiple cross coupled paths. But for simplicity only cross coupling across three resonators is used to design ceramic waveguide inductive and capacitive triplets.

\subsubsection{Inductive cross coupled triplet}

To produce a transmission zero above pass band of the filter, a cross coupled path is created from resonator 1 to resonator 3, as shown in Figure 5.6. The direct coupling between resonators 1-2 and 2-3 is realized by a circular metal plated through hole placed at the intersection of all three resonators. Three metal plated through holes are placed between resonator 1 and resonator 3 to allow the weak cross coupling. The amount of coupling needed to realize right coupling bandwidth can be adjusted by the diameter of the through holes and their offset from the side wall. Through holes in the 
ceramic waveguide are placed symmetrically in order to supress higher order modes.

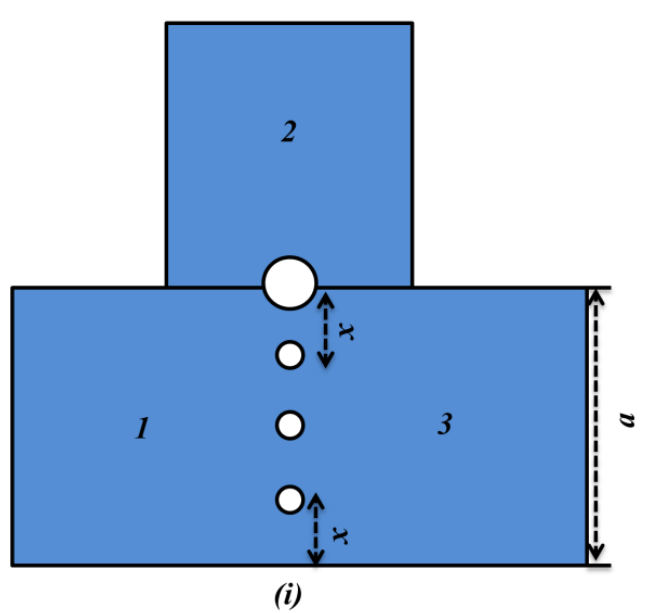

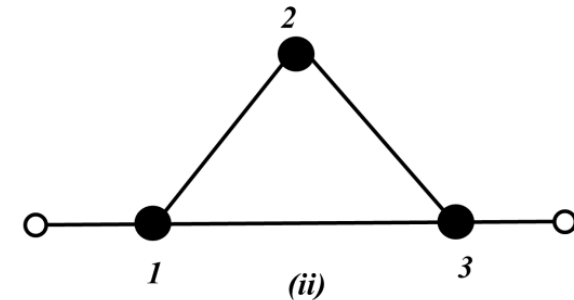

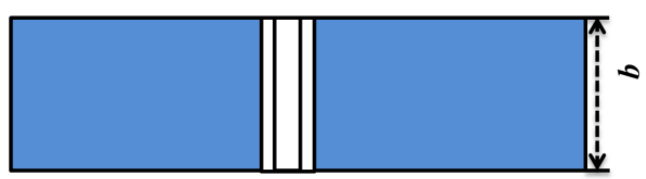

(iii)

Figure 5.6: Ceramic waveguide inductive triplet (i) Top view (ii) Coupling scheme (iii) Side view

Figure 5.7 shows the response of an inductive triplet of ceramic waveguide filter with different cross coupled bandwidths.

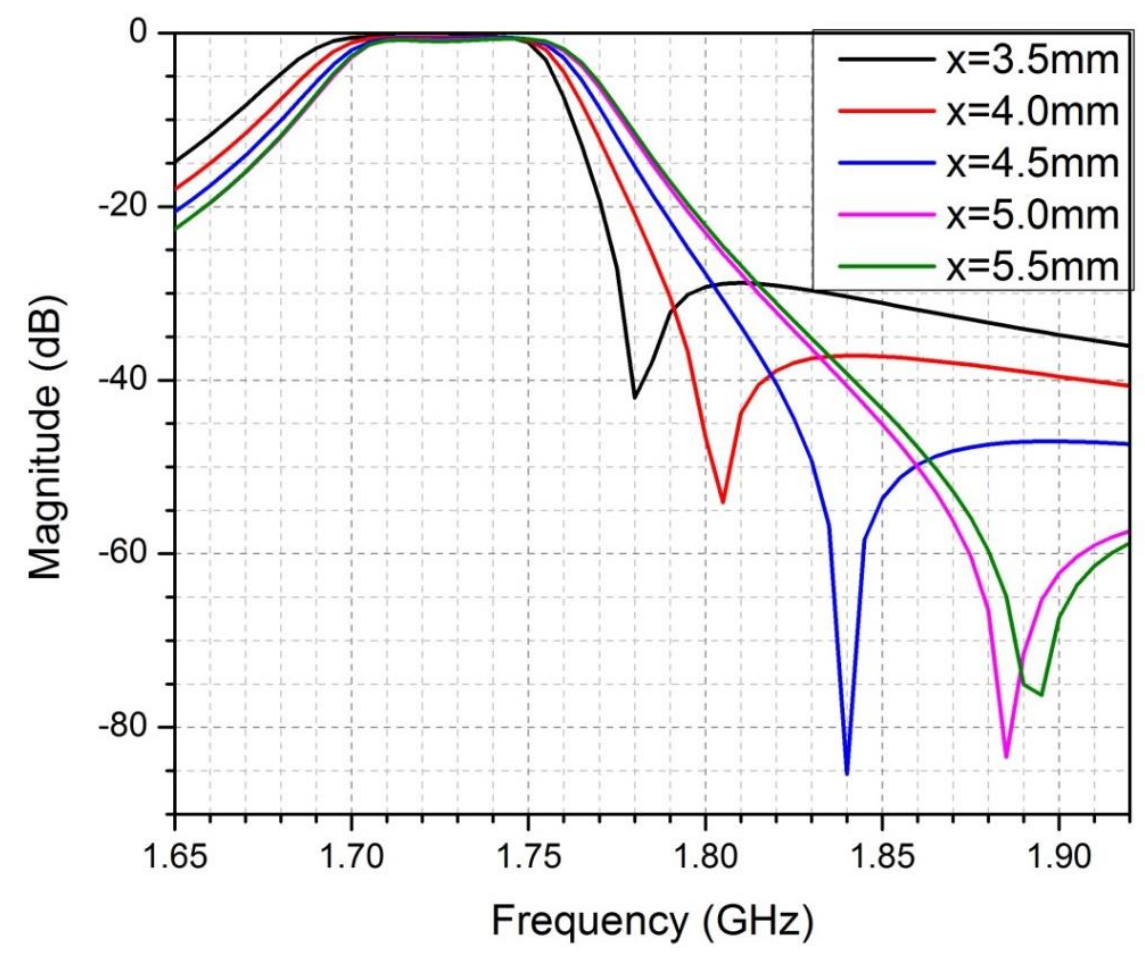

Figure 5.7: High side transmission zero control by varying inductive cross coupling 
The position of transmission zero is controlled by the radius and offset of metal plated holes from side walls. These holes behave as a shunt inductive impedance inverter between ceramic waveguide half wavelength resonators over a broad bandwidth. It is observed that the offset of hole centres placed between resonator 1 and resonator 3 control the position of transmission zero. As the offset of the coupling holes is increased the amount of coupling is decreased and the location of transmission zero is shifted away from the passband.

\subsubsection{Capacitive cross coupled triplet}

To produce a transmission zero at lower side of the passband of the filter, a capacitive cross coupling is required between non-adjacent resonators. A silver plated blind hole at the centre of the broad wall of ceramic waveguide behaves as shunt capacitor between two ceramic waveguide resonators as shown in Figure 5.8. The direct couplings from resonator 1-2 and 2-3 are realized by a through hole at the intersection of all three resonators. And to produce a capacitive coupling between resonators 1-3, a metal plated blind hole is placed between them. The amount of coupling required to realize right coupling bandwidth is a function of blind hole diameter and its depth inside the ceramic waveguide. The resonating nature of the coupling structure $[65,70,71]$ produces an extra transmission zero above the pass band of the filter. This extra transmission zero can be placed away from the pass band by varying the diameter of the blind hole, so as not to degrade the performance of the filter in the pass band.
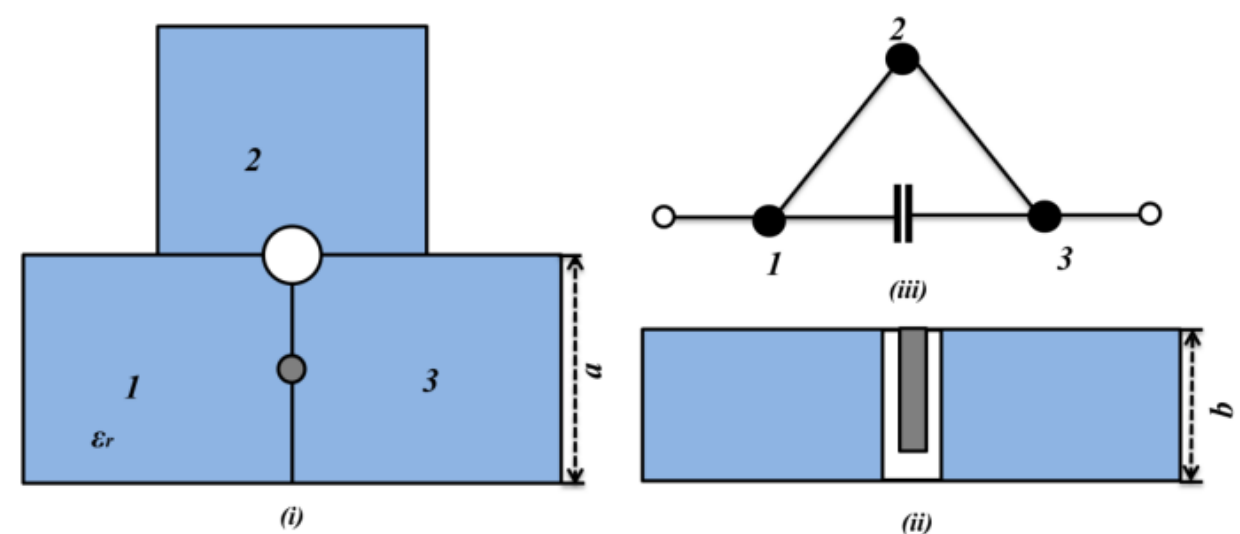

(ii)

Figure 5.8: Ceramic waveguide capacitive triplet (i) Top view (ii) Side view

(iii) Coupling scheme 
Figure 5.9 shows a capacitive cross coupled triplet response with different $\mathrm{h} / \mathrm{b}$ ratio where ' $b$ ' represents the height of the waveguide and ' $h$ ' is referred to the depth of the blind hole inside the waveguide. It is evident from the Figure 5.9 that for a fixed diameter coupling hole, the coupling bandwidth decreases by increasing the depth of the hole and therefore transmission zero moves away from the passband of the filter.

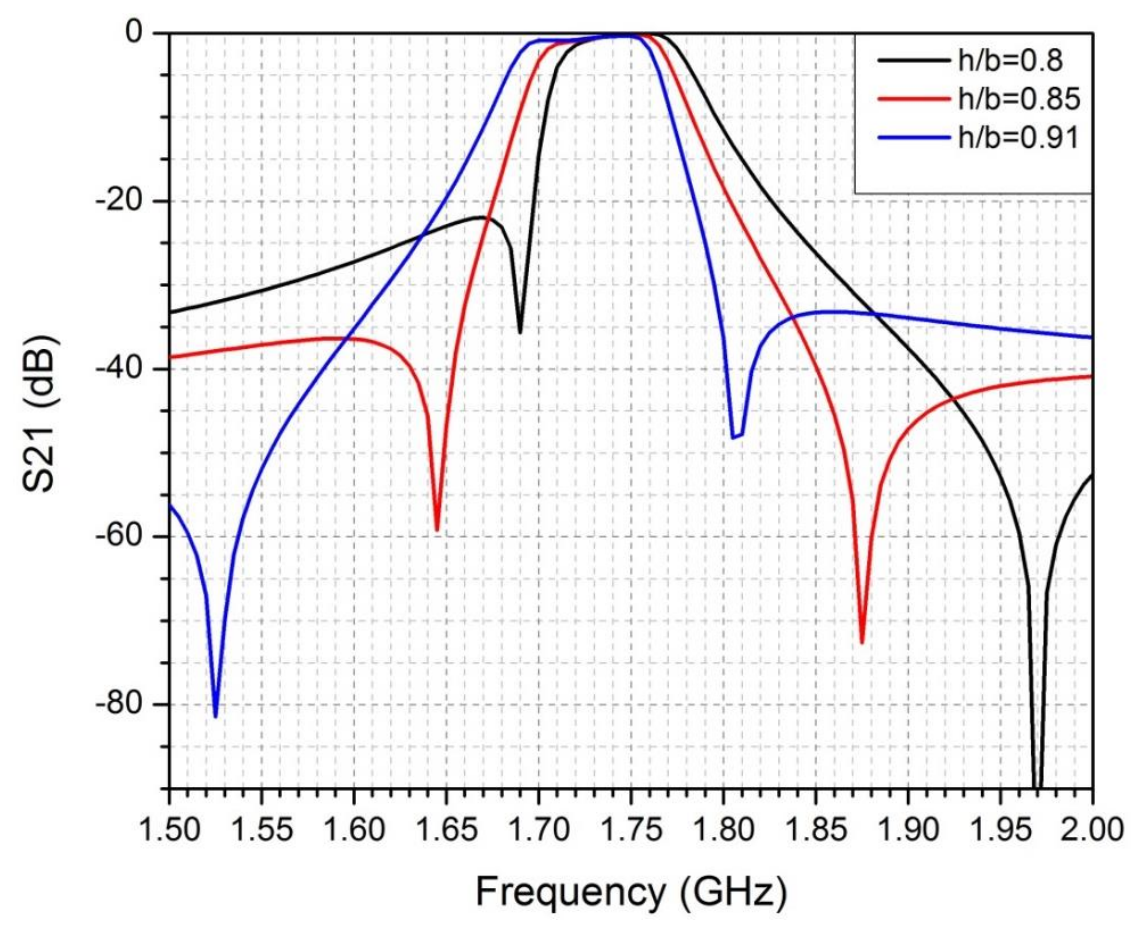

Figure 5.9: Low side transmission zero control by varying capacitive cross coupling

\subsection{External coupling}

To achieve external couplings in a rectangular ceramic waveguide filter, a coaxial probe is placed at the high E-field region of the first and last resonator of the filter as explained in section 4.6. The inner conductor of coaxial probe acts as an antenna to excite the energy inside the ceramic waveguide. Electric field has its maximum value at the centre of the broad wall in the ceramic rectangular waveguide; therefore a probe placed at this position excites strong E-field inside the waveguide.

\subsection{Generalized Chebyshev filter design}

A six pole Chebyshev ceramic waveguide filter was designed and explained in Chapter 4. A more complex design of a six section cross coupled ceramic waveguide filter 
operating at digital cellular system (DCS) uplink frequency band is designed using cross coupled triplets to meet the following specifications.

Table 5-4: Ceramic waveguide generalised Chebyshev filter design parameters

\begin{tabular}{|l|l|}
\hline Dielectric Constant & 43 \\
\hline Dielectric Loss Tangent & 0.00004 \\
\hline Silver Conductivity & $4.4 \times 10^{7} \Omega^{-1} \mathrm{~m}^{-1}$ \\
\hline System Impedance & $50 \Omega$ \\
\hline Centre frequency & $1730 \mathrm{MHz}$ \\
\hline Passband Bandwidth & $60 \mathrm{MHz}$ \\
\hline Passband Insertion loss & $\leq 0.5 \mathrm{~dB}$ \\
\hline Order of the filter & 6 \\
\hline Passband Return loss & $\geq 20 \mathrm{~dB}$ \\
\hline Stopband attenuation & $\geq 75 \mathrm{~dB}$ at $\mathrm{DC}<\mathrm{f}<1640 \mathrm{MHz}$ \\
& $\geq 75 \mathrm{~dB}$ at $1830 \mathrm{MHz}<\mathrm{f}<2300 \mathrm{MHz}$ \\
\hline
\end{tabular}

\subsubsection{Filter with transmission zero above pass band}

A six section generalized Chebyshev filter with a transmission zero above pass band is designed based on the coupling matrix given in Figure 5.10. The filter pass band bandwidth is $60 \mathrm{MHz}$ and the passband centre frequency is $1730 \mathrm{MHz}$.

$\left[\begin{array}{ccccccccc}\cdot & S & 1 & 2 & 3 & 4 & 5 & 6 & L \\ S & 0 & 1.002 & 0 & 0 & 0 & 0 & 0 & 0 \\ 1 & 1.002 & 0.0043 & 0.8358 & 0.1084 & 0 & 0 & 0 & 0 \\ 2 & 0 & 0.8358 & -0.1515 & 0.604 & 0 & 0 & 0 & 0 \\ 3 & 0 & 0.1084 & 0.604 & 0.023 & 0.5833 & 0 & 0 & 0 \\ 4 & 0 & 0 & 0 & 0.5833 & 0.0092 & 0.6109 & 0 & 0 \\ 5 & 0 & 0 & 0 & 0 & 0.6109 & 0.0055 & 0.8428 & 0 \\ 6 & 0 & 0 & 0 & 0 & 0 & 0.8428 & 0.0043 & 1.002 \\ L & 0 & 0 & 0 & 0 & 0 & 0 & 1.002 & 0\end{array}\right]$

Figure 5.10: Coupling matrix of generalised Chebyshev filter with one cross coupling

All positive couplings in a ceramic waveguide are achieved by silver plated through 
holes. A weak cross coupled path is introduced between resonators 1-3 to produce an attenuation pole at a frequency of $1880 \mathrm{MHz}$. Figure 5.11 shows the coupling diagram of the generalized Chebyshev bandpass filter represented by coupling matrix shown in Figure 5.10.

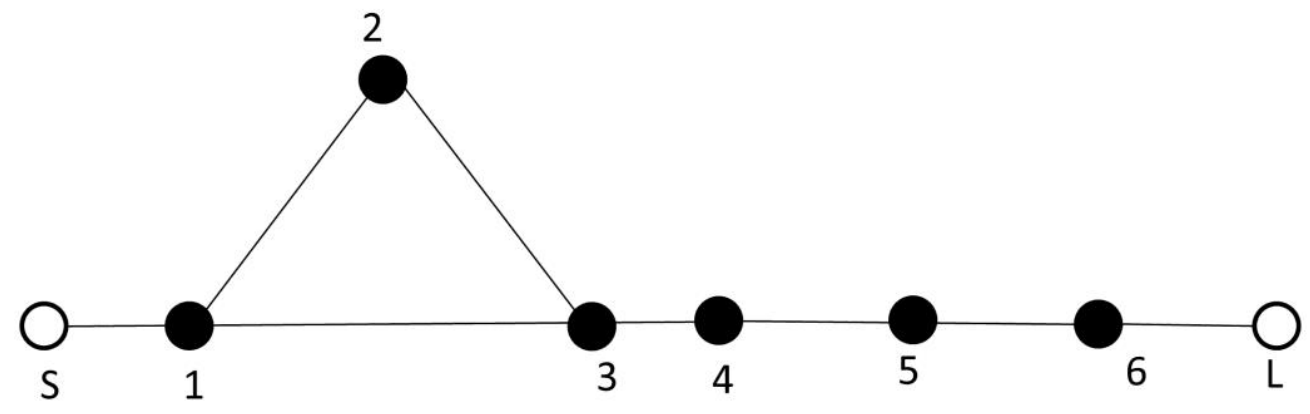

Figure 5.11: Generalized Chebyshev filter configuration with an inductive triplet

Figure 5.12 represents the physical layout of the generalised Chebyshev ceramic waveguide bandpass filter with an attenuation pole at $1880 \mathrm{MHz}$. And its S-parameter response is shown in Figure 5.13. The position of the attenuation pole frequency can be adjusted by varying the cross coupled path coupling bandwidth.
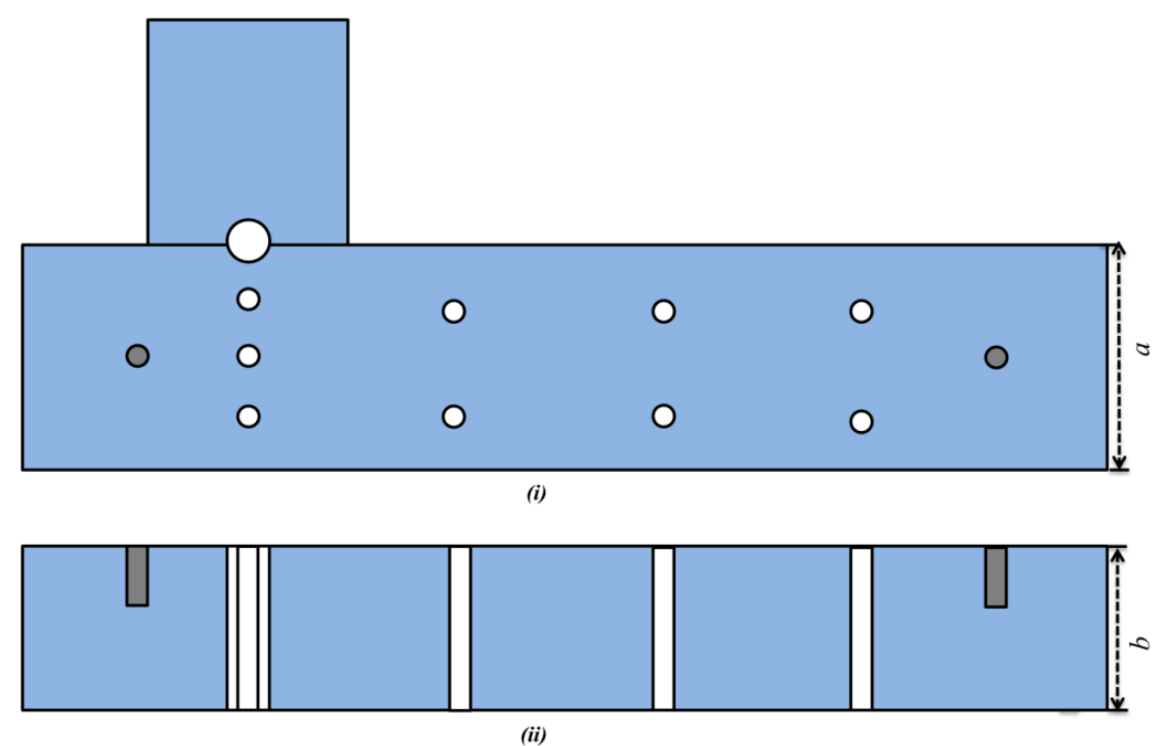

Figure 5.12: Generalized Chebyshev ceramic waveguide filter layout with an inductive triplet (i) Top view (ii) Side view 


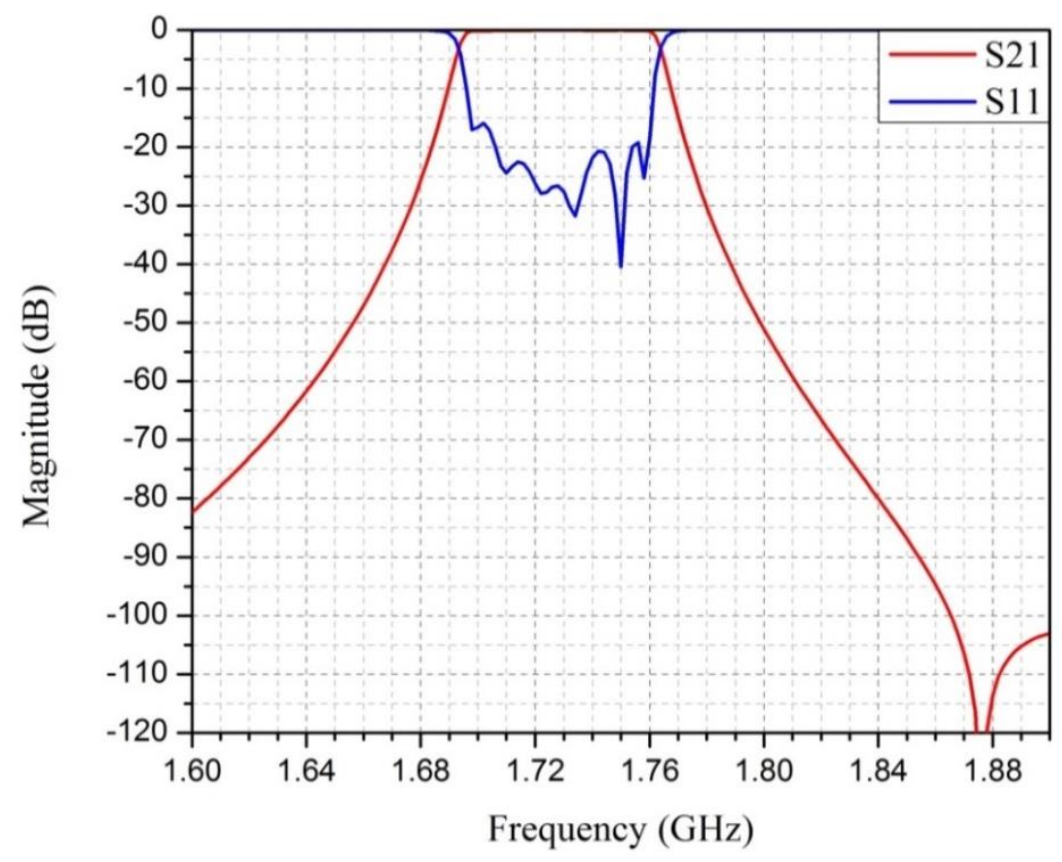

Figure 5.13: A generalised Chebyshev ceramic waveguide filter with Transmission zero above passband (HFSS ${ }^{\mathrm{TM}}$ simulation)

\subsubsection{Filter with transmission zero below pass band}

A generalised Chebyshev bandpass filter with a capicitively coupled triplet to produce a transmission zero below passband is designed. The coupling matrix for a generalised Chebyshev filter with a transmission zero below passband is shown in Figure 5.14. The coupling scheme of the filter is shown in Figure 5.15, which represents all direct or main line couplings are positive while capacitive coupling is introduced between resonators 4-6 to realize the attenuation pole at $1645 \mathrm{MHz}$.

$$
\left[\begin{array}{ccccccccc}
\cdot & S & 1 & 2 & 3 & 4 & 5 & 6 & L \\
S & 0 & 1.0018 & 0 & 0 & 0 & 0 & 0 & 0 \\
1 & 1.0018 & -0.0069 & 0.8425 & 0 & 0 & 0 & 0 & 0 \\
2 & 0 & 0.8425 & -0.0088 & 0.6107 & 0 & 0 & 0 & 0 \\
3 & 0 & 0 & 0.6107 & -0.014 & 0.5832 & 0 & 0 & 0 \\
4 & 0 & 0 & 0 & 0.5832 & -0.0363 & 0.5931 & -0.1727 & 0 \\
5 & 0 & 0 & 0 & 0 & 0.5931 & 0.2408 & 0.8246 & 0 \\
6 & 0 & 0 & 0 & 0 & -0.1727 & 0.8246 & -0.0069 & 1.0018 \\
L & 0 & 0 & 0 & 0 & 0 & 0 & 1.0018 & 0
\end{array}\right]
$$

Figure 5.14: Coupling matrix for a generalised Chebyshev filter with negative cross coupling 


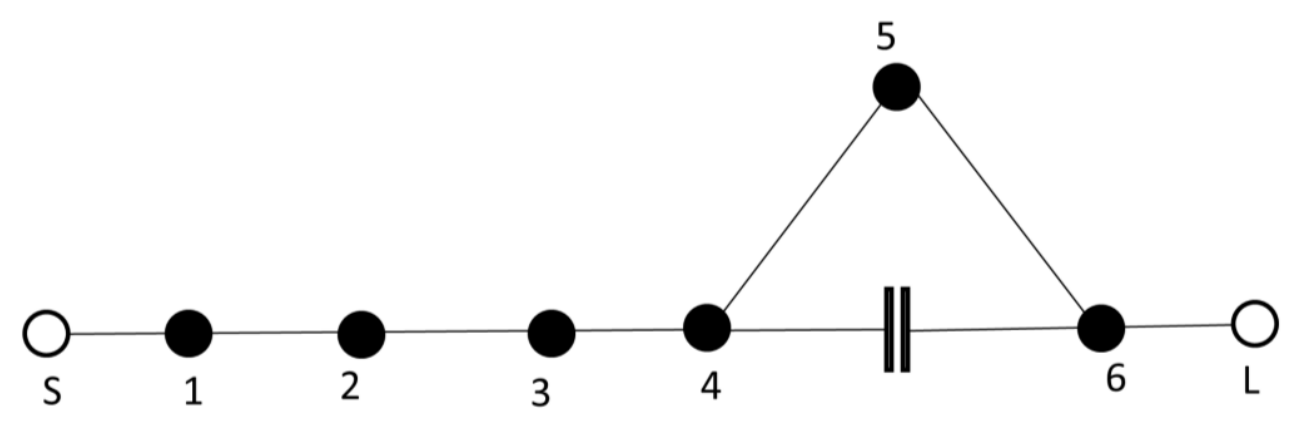

Figure 5.15: Generalized Chebyshev filter configuration with a capacitive triplet

Figure 5.16 represents the physical layout of the generalised Chebyshev ceramic waveguide bandpass filter with an attenuation pole at $1645 \mathrm{MHz}$. The capacitive cross coupling is realized by placing a blind hole between resonators 4-6. The s-parameter response of the filter is shown in Figure 5.17. It is evident from Figure 5.17 that besides a transmission zero at $1645 \mathrm{MHz}$, another attenuation pole is introduced at a higher frequency $1890 \mathrm{MHz}$ due to resonating nature of the blind hole coupling structure. The position of this spurious attenuation pole can be moved away from the passband by selecting the appropriate diameter of the blind hole coupling structure.

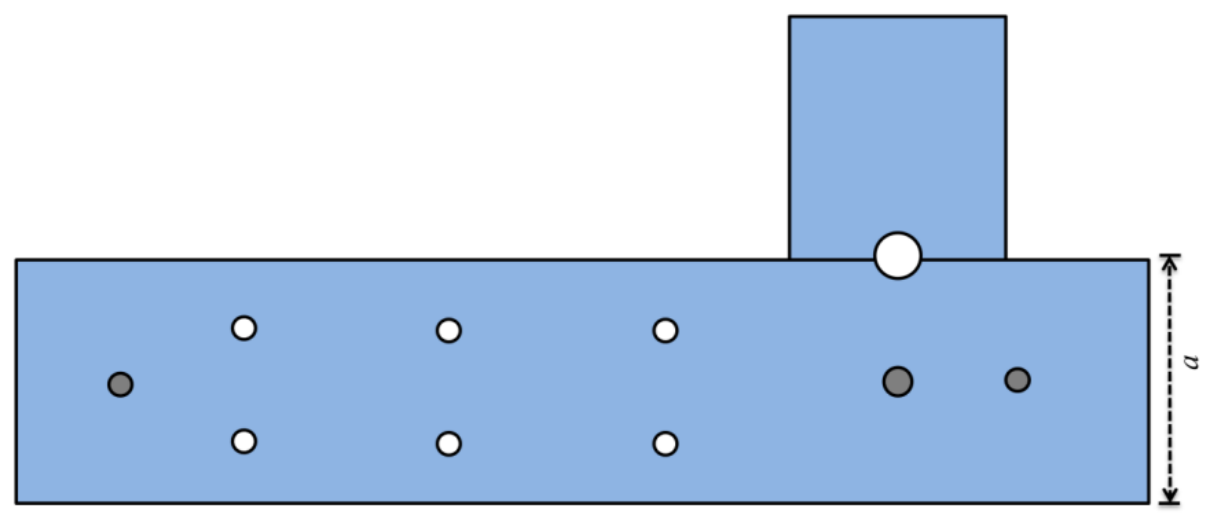

(i)

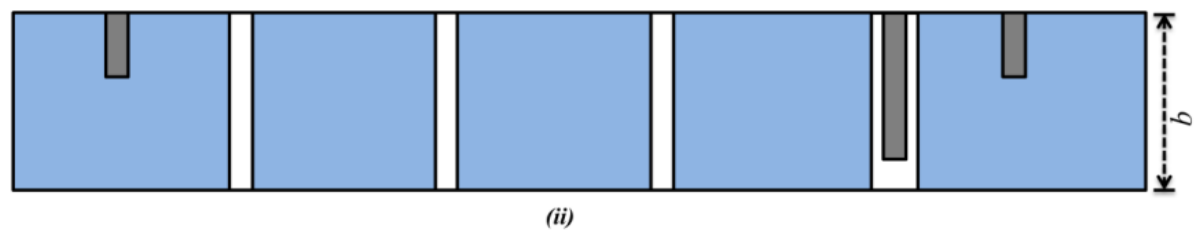

Figure 5.16: Generalized Chebyshev ceramic waveguide filter layout with capacitive cross coupled triplet (i) Top view (ii) Side view 


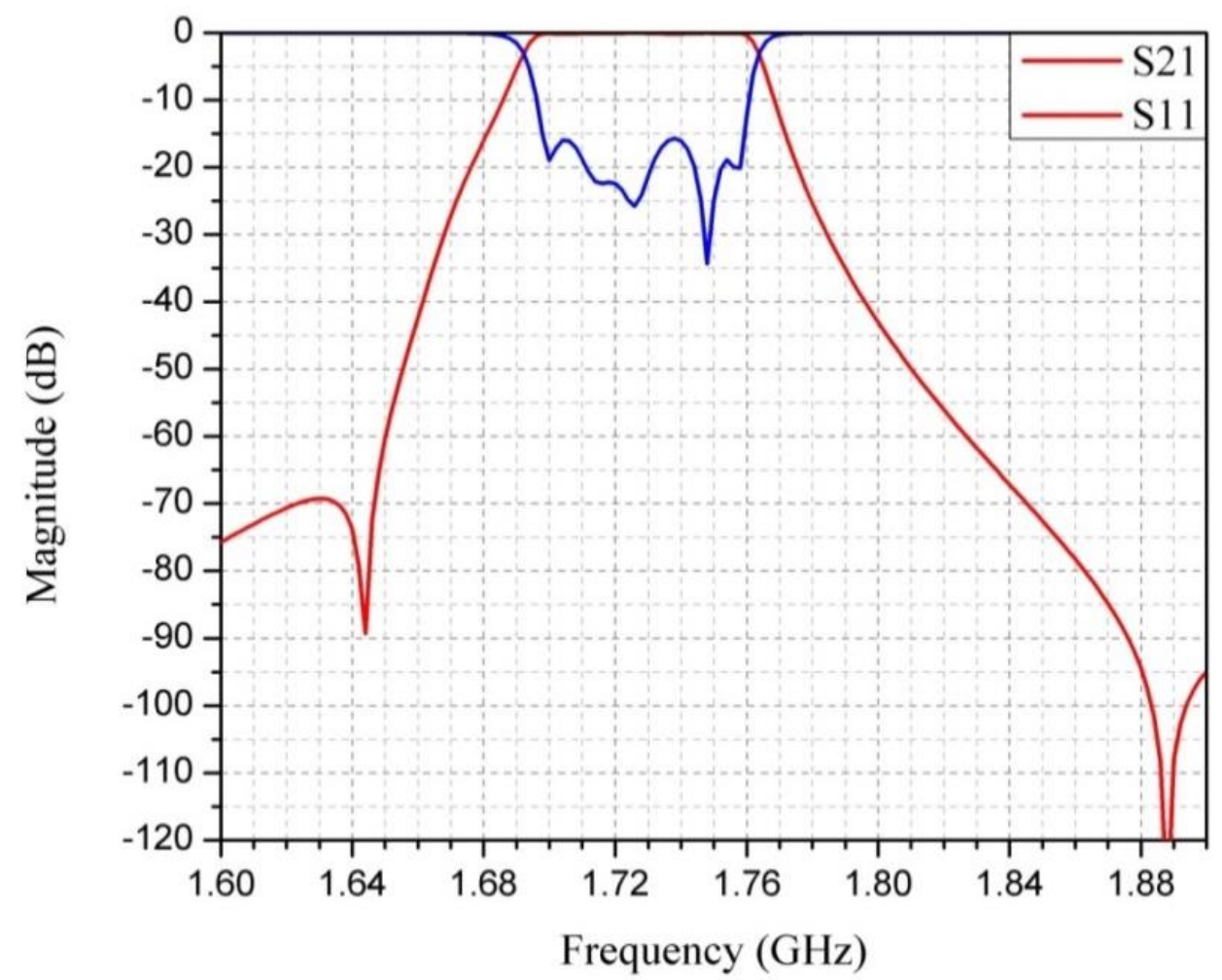

Figure 5.17: Generalised Chebyshev ceramic waveguide bandpass filter simulated response with a transmission zero below passband $\left(\mathrm{HFSS}^{\mathrm{TM}}\right)$

\subsubsection{Filter with transmission zeros above and below pass band}

A generalised Chebyshev ceramic waveguide bandpass filter with a bandwidth of 60 $\mathrm{MHz}$ at a centre frequency of $1730 \mathrm{MHz}$ and attenuation pole on both sides of the passband is designed. The coupling matrix is given in Figure 5.18 and coupling scheme is shown in Figure 5.19. The six section filter consists of a combination of an inductive and a capacitive cross coupled triplet. Inductive cross coupling produces a transmission zero above the passband and attenuation pole below the passband is realized via capacitive cross coupling between non-adjacent resonators. All positive couplings in the ceramic waveguide are realized via metal plated through holes in the broad wall of the waveguide. Negative coupling between resonators 4-6 is achieved by placing a metal plated blind hole at the centre of the broad wall of waveguide. However, the blind hole resonates at a higher frequency and above resonance becomes inductive producing a further transmission zero on the high frequency side of the pass band. Position of this extra transmission zero can be controlled by selecting the appropriate diameter and 
depth of the blind hole. The input and output couplings are achieved by $50 \mathrm{ohm}$ coaxial probes. The Probe diameter, depth inside the waveguide, its distance from the shorted backend and offset from centre determines its coupling bandwidth, power handling and centre frequency.

$$
\left[\begin{array}{ccccccccc} 
& S & 1 & 2 & 3 & 4 & 5 & 6 & L \\
S & 0 & 1.0626 & 0 & 0 & 0 & 0 & 0 & 0 \\
1 & 1.0626 & -0.0035 & 0.8897 & 0.1218 & 0 & 0 & 0 & 0 \\
2 & 0 & 0.8897 & -0.1749 & 0.6232 & 0 & 0 & 0 & 0 \\
3 & 0 & 0.1218 & 0.6232 & 0.0101 & 0.5993 & 0 & 0 & 0 \\
4 & 0 & 0 & 0 & 0.5993 & -0.0341 & 0.6097 & -0.1969 & 0 \\
5 & 0 & 0 & 0 & 0 & 0.6097 & 0.2713 & 0.8761 & 0 \\
6 & 0 & 0 & 0 & 0 & -0.1969 & 0.8761 & -0.0035 & 1.0626 \\
L & 0 & 0 & 0 & 0 & 0 & 0 & 1.0626 & 0
\end{array}\right]_{\text {? }}
$$

Figure 5.18: Coupling matrix of generalised Chebyshev BPF with a capacitive and an inductive cross coupled triplet

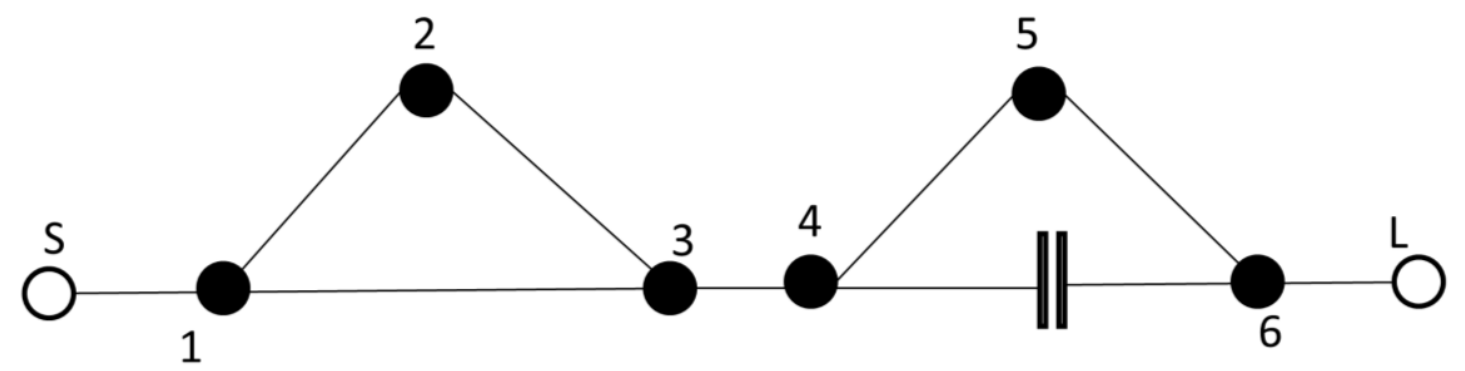

Figure 5.19: Generalized Chebyshev filter configuration with a capacitive and an inductive cross coupled triplet

Figure 5.20 shows the final layout of the cross coupled generalized Chebyshev filter designed to operate at DCS uplink frequency band. The response of generalized Chebyshev filter designed is shown in Figure 5.21. A closer view of passband insertion loss is also given which suggests that IL is less than $0.8 \mathrm{~dB}$ in the passband of the filter. 

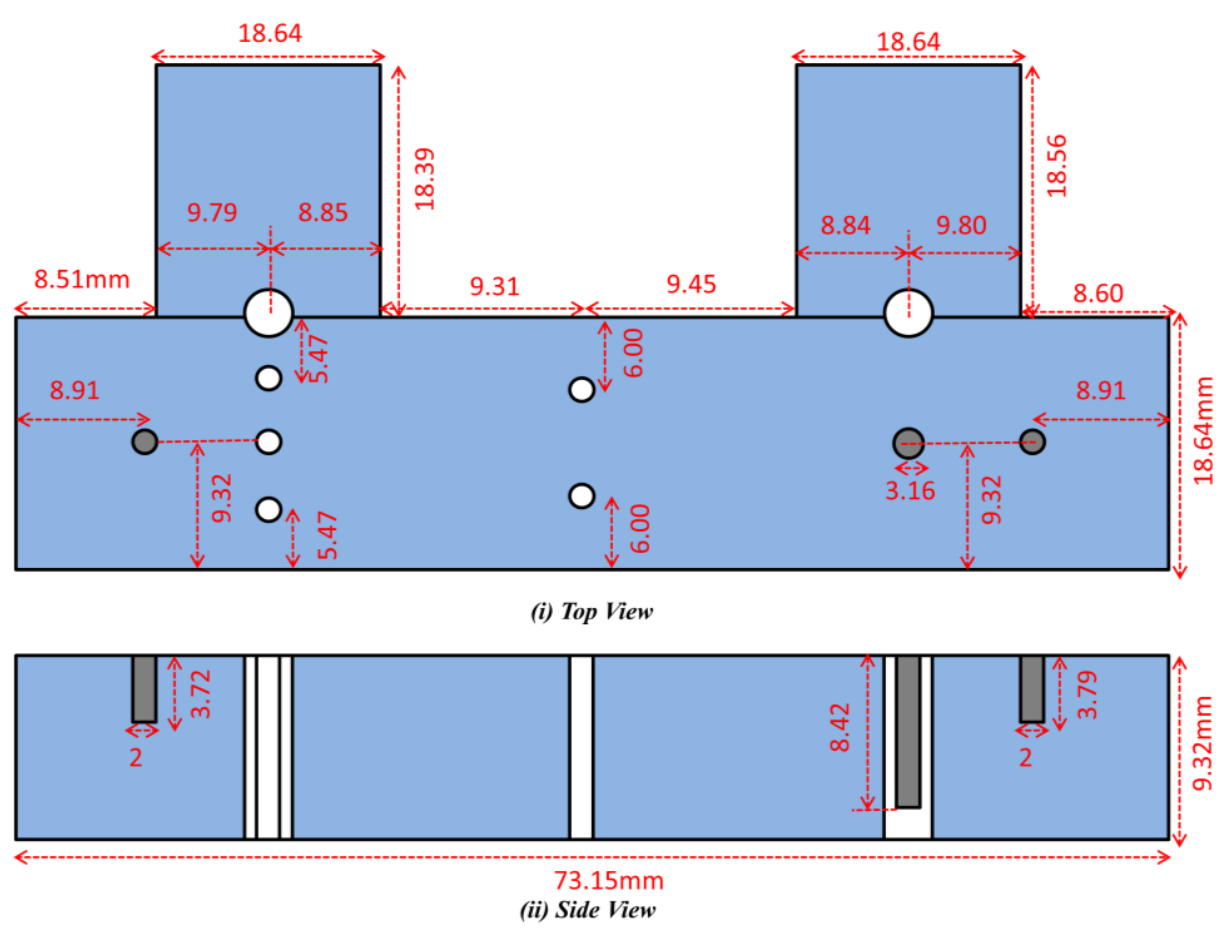

Figure 5.20: Ceramic waveguide generalised Chebyshev bandpass filter drawing

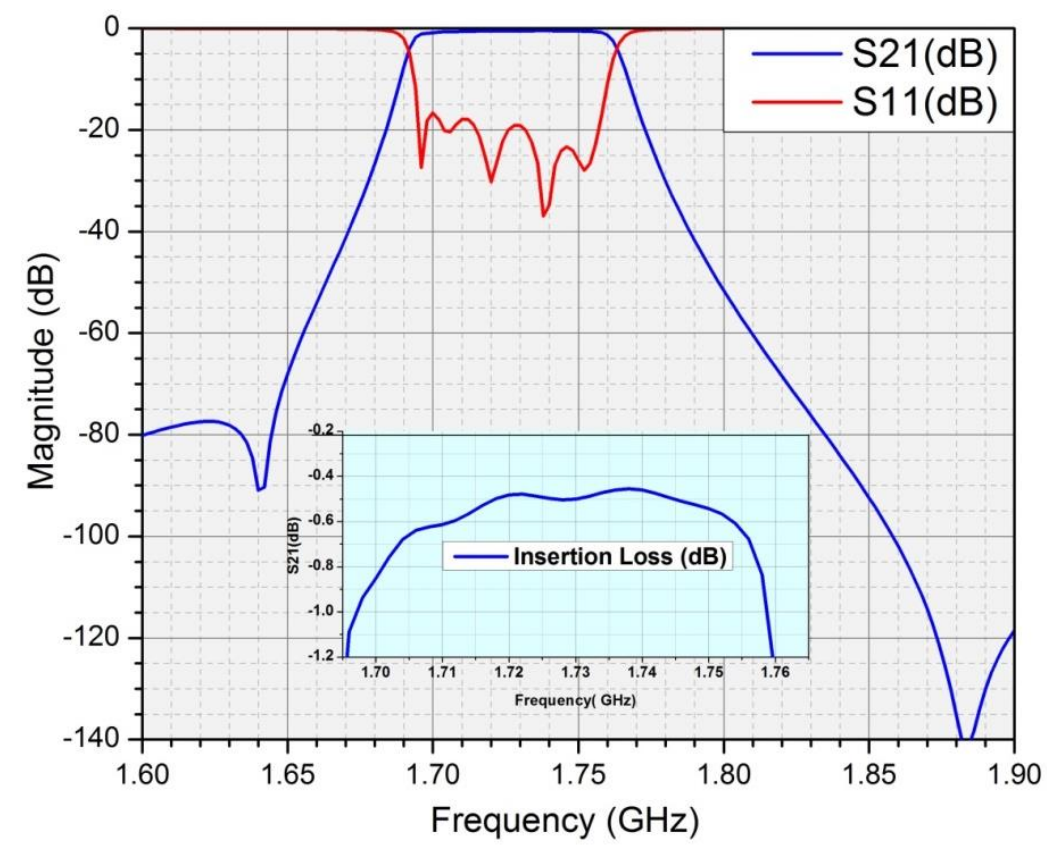

Figure 5.21: Simulated RX filter with Transmission zero below and above passband $\left(\mathrm{HFSS}^{\mathrm{TM}}\right)$ 
Figure 5.22 shows the photograph of the fabricated ceramic waveguide filter. A comparison of measured results and HFSS ${ }^{\mathrm{TM}}$ simulation is given in Figure 5.23.

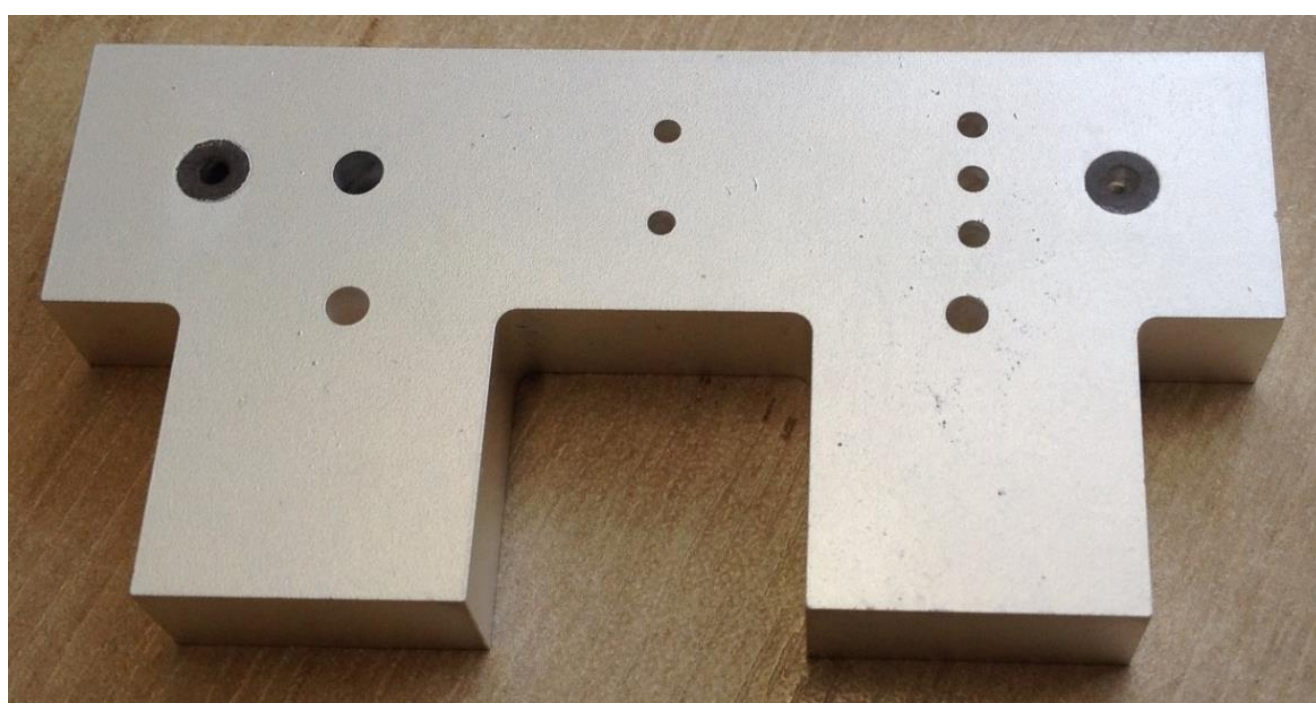

Figure 5.22: Fabricated ceramic waveguide RX filter

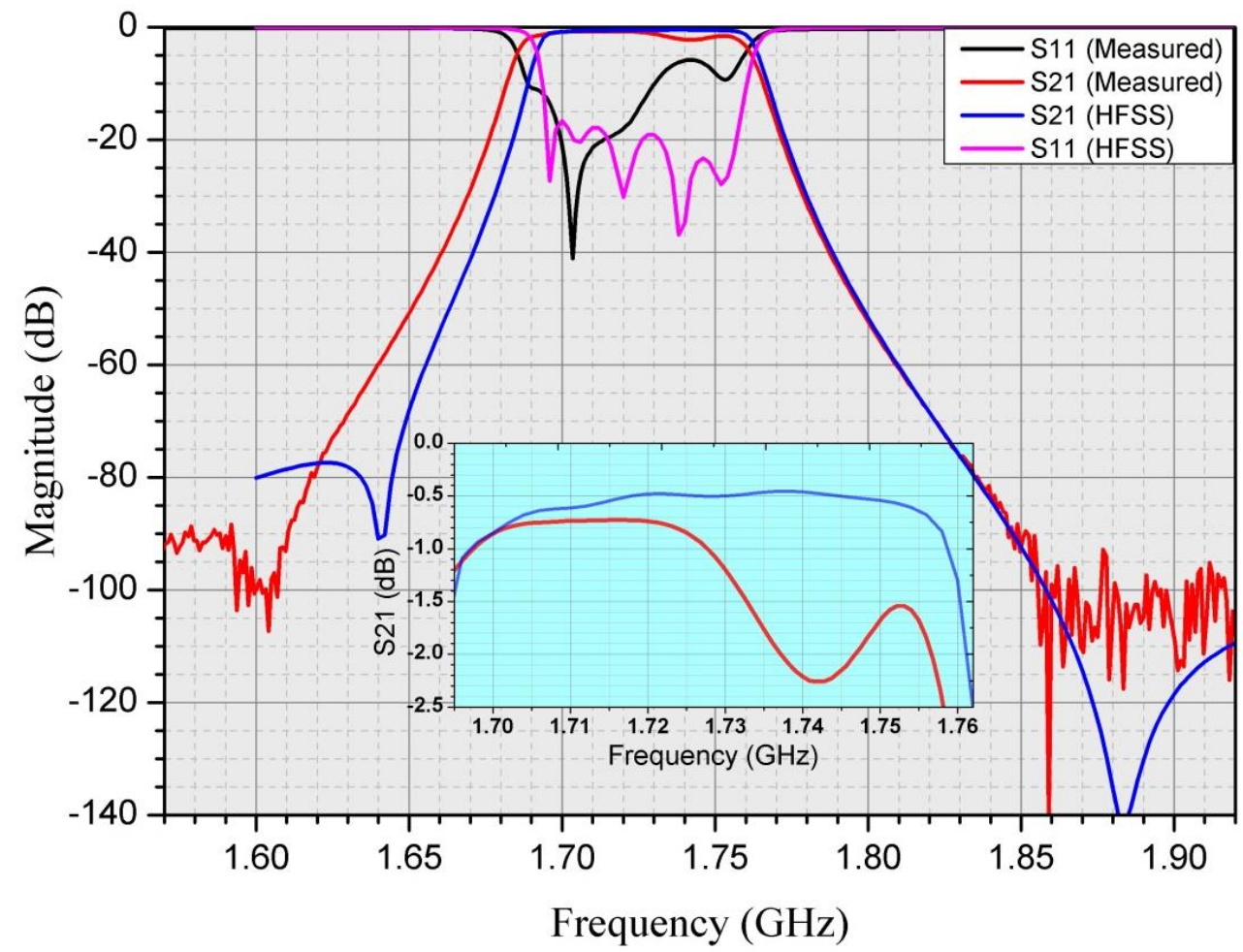

Figure 5.23 : Measured and HFSS ${ }^{\mathrm{TM}}$ simulated response 
The passband return loss and insertion loss of measured filter is not as expected due to the presence of curved radii of side resonators in the physical design, which are not included in EM simulations. Also the lower side transmission zero is moved further away from passband, due to change in resonance of side resonators of the filter. Measurement shows that bandwidth of the passband is increased, the overall passband is shifted to the lower side and a severe mismatch occurs at the higher side of the passband. This is due to inclusion of curved radius of the side resonators, which is not included in EM simulation. In Figure 5.24, the measured results are compared with an EM simulation of the filter which includes the curved radii of side resonators. The graph shows a close agreement of both measured and simulated results. The high side transmission zero is not clearly visible in measured response as it is buried in the noise floor. The passband insertion loss is about $0.7 \mathrm{~dB}$ in the region of passband where filter is well matched. The return loss and the transmission zero positions are corrected in the next design with tuning screws.

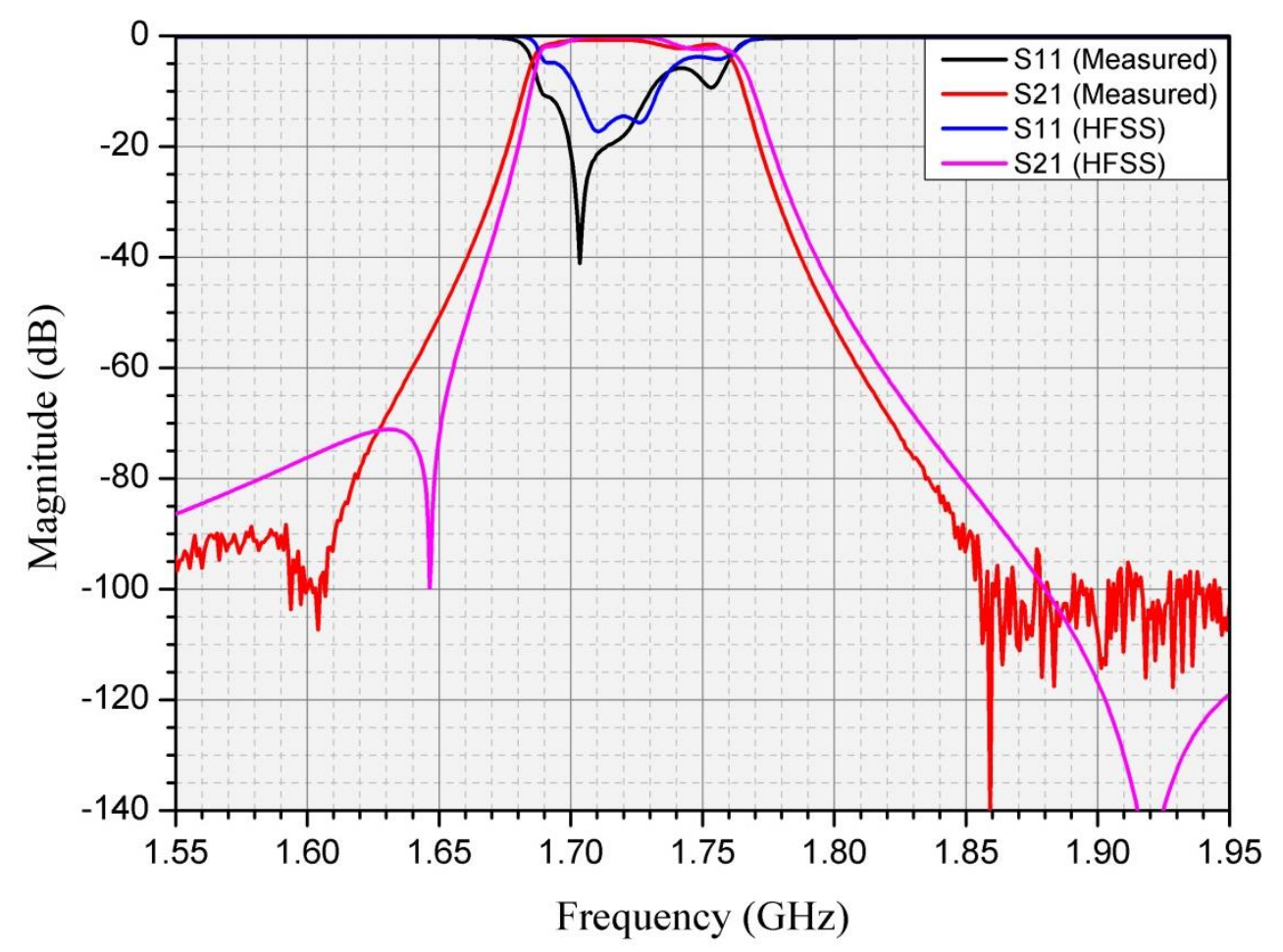

Figure 5.24 : Measured and EM simulated response with side resonator radii included 


\subsubsection{Transmission zero control}

Ceramic waveguide filter discussed in 5.7.3 has three transmission zeros at $1640 \mathrm{MHz}$, $1880 \mathrm{MHz}$ and $1882 \mathrm{MHz}$. The transmission zero produced below the pass band is due to capacitive cross coupling path formed between resonator 4 and 6 . The first transmission zero above the pass band is achieved by forming inductive cross coupled path between resonator 1 and 3. The third transmission zero is caused due to spurious resonance of coupling structure between resonator 4 and 6 . The position of transmission zeros above or below the pass band can be controlled independently. Although both of the transmission zero below the pass band and spurious transmission zero depend on the physical dimensions of the same coupling structure, i.e. C4-6, but they can be adjusted at appropriate positions. The first transmission zero above the pass band is dependent on the cross coupling between resonator 1 and 3 and its position is dependent on the amount of coupling allowed through cross coupled path.

Figure 5.25 shows the response of a generalised Chebyshev bandpass filter with high side transmission zero moved near to the passband to produce an attenuation pole at a frequency of $1810 \mathrm{MHz}$ by increasing the amount of inductive cross coupling.

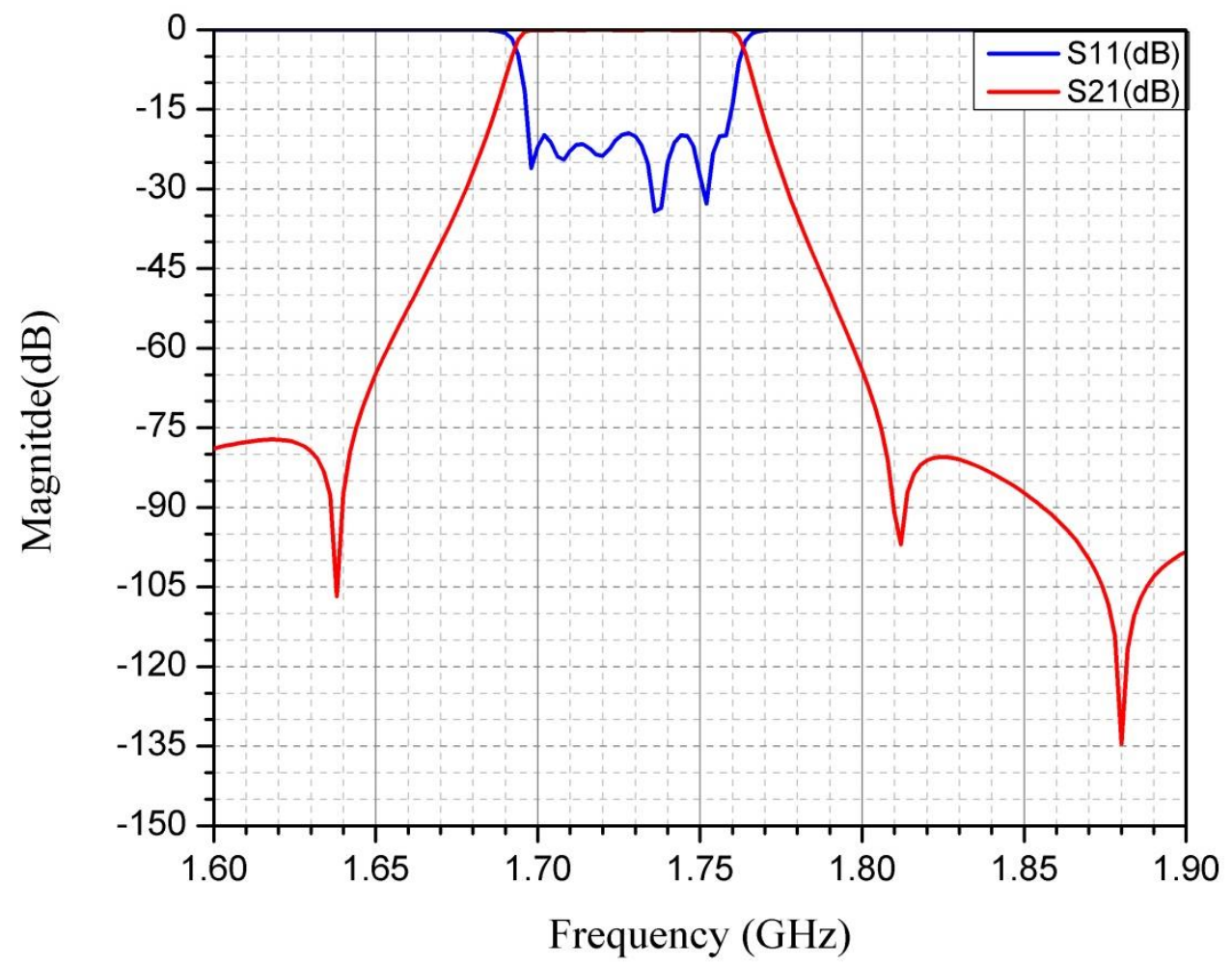


Figure 5.25: RX filter with transmission zero near pass-band

To achieve a transmission zero near to pass band edge, cross coupled path is required to allow more energy through it. Thus a full control of transmission zero above pass-band can be achieved by changing the amount of inductive coupling through cross coupled path. In a ceramic waveguide filter, the amount of inductive coupling allowed between two resonators is dependent on the radius of silver plated through holes in the broad wall and their distance from the side walls.

\subsubsection{Generalized Chebyshev filter with tuning screws}

To compensate any frequency shift in the response of fabricated filter due to physical design tolerances and any temperature shift or material imperfection, a post production tuning mechanism is often required. There could be several possibilities to tune the response of a ceramic rectangular waveguide filter, which are discussed in detail in Chapter.8. Here, the metal tuning screws are placed at the bottom broad wall of the filter in each resonator section. All resonators are deployed with tuning screws at the middle of the cavity where the maximum E-field occurs except first and last cavity. These cavities are excited with external probes at the centre of broad wall which could possibly be perturbed due to tuning screw presence. Thus, in first and last resonator tuning screws are placed midway between centre of the broad wall and side wall so as to keep input coupling unaffected when tuning resonant cavity only. Figure 5.26 shows the layout of RX filter with tuning screws. The waveguide filter is simulated with tuning screws half way inside the tuning hole, so as to keep the option of both way post production tuning mechanism. The non-metalized blind holes placed in the ceramic waveguide to accommodate the metal tuning screws inside the waveguide shift resonance frequency of ceramic rectangular waveguide resonator to a higher value. The amount of tuning achieved is a function of blind hole depth inside the waveguide, tuning screw depth inside the hole, radius of blind hole and radius of tuning screw. Detailed discussion and data with different radius and depth of tuning screw and blind hole is given in Chapter 8. It is desired to have dimensions of blind hole to be small as Q-factor of the resonator decreases by increasing the depth of the blind hole. The physical dimensions of the resonators need to be corrected first with a tuning screw half way inside the resonator before designing the filter. Figure 5.27 shows the simulated 
response of a six section cross coupled generalized Chebyshev ceramic waveguide filter with tuning screws mounted in it.
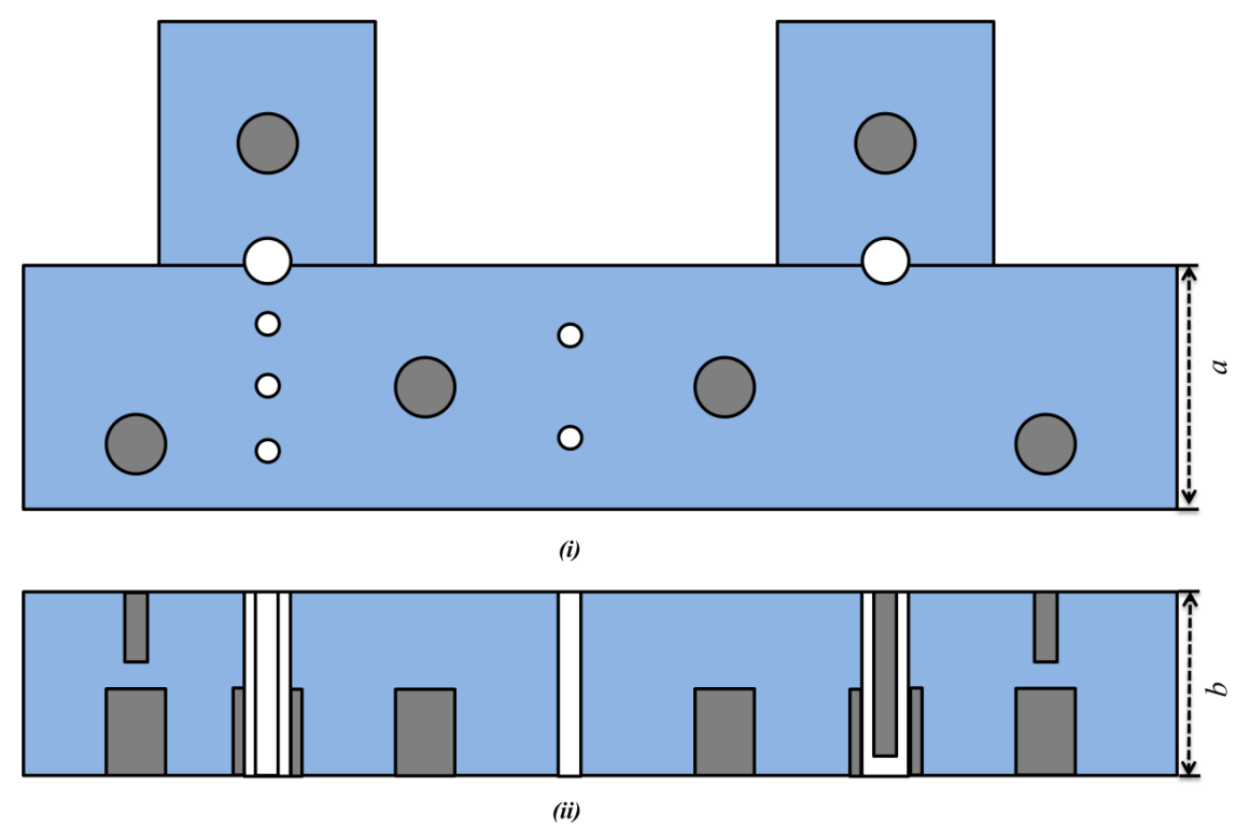

Figure 5.26: Ceramic waveguide filter with tuning screws (i) bottom view (ii) Side view

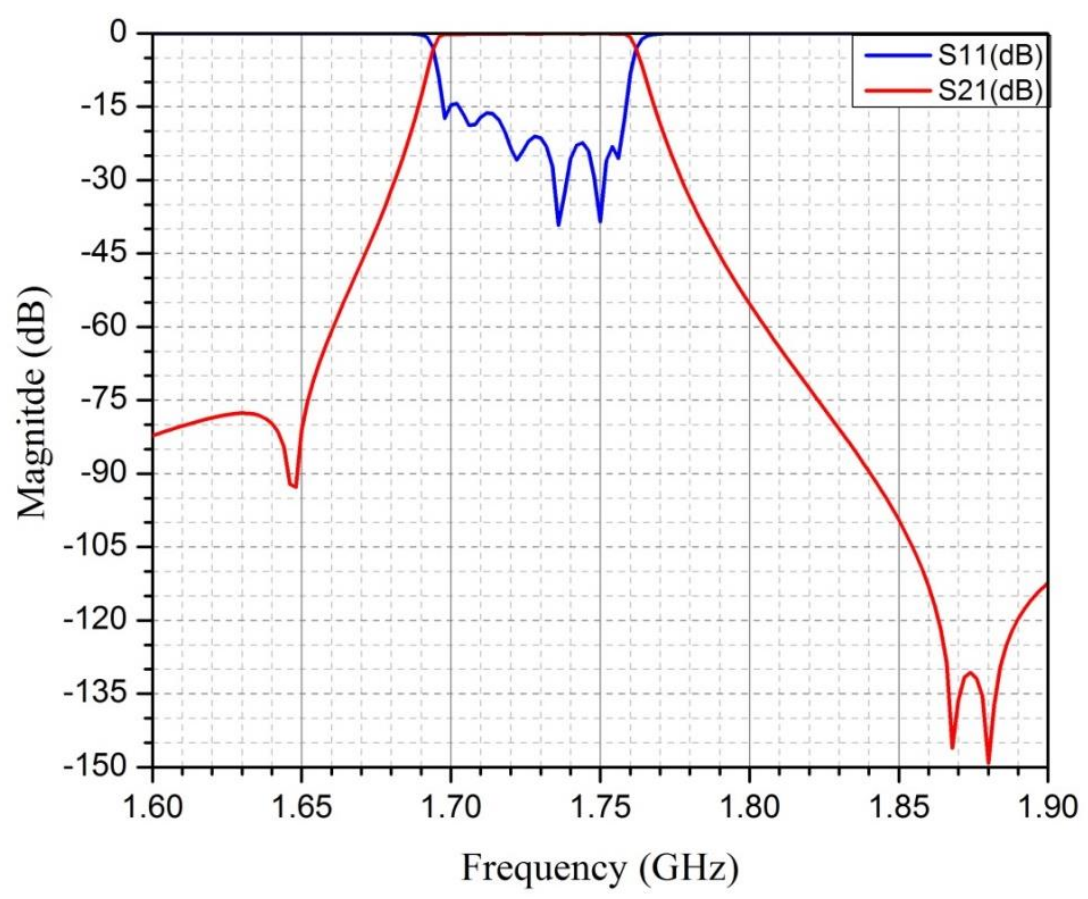

Figure 5.27: Simulated generalised Chebyshev ceramic WG BPF with tuning screws $\left(\mathrm{HFSS}^{\mathrm{TM}}\right)$ 
A fabricated generalized Chebyshev ceramic waveguide filter having transmission zeros at above and below the pass band and with tuning screws in it is shown in Figure 5.28. A comparison of its simulated and measured results is shown in Figure 5.29. It is evident from Figure 5.29 that the position of transmission zero at lower side of the passband is shifted towards the passband also insertion loss in the passband of the filter is higher than expected, as the return loss is worse as compared to simulated results. Although a full control over resonant frequencies can be achieved independently using tuning screws but still the performance of the filter is not satisfactory. The main reason behind this degraded performance is the inclusion of curved radii of side resonators in physical design which were not included in the simulations. Due to the inclusion of the side radii in physical filter, the resonance and coupling of side resonators has changed. The resonance of these side resonators can be optimised using tuning screws but unfortunately, the coupling bandwidth of side resonators is not tuneable in this case. Therefore, due to mismatch worse return loss and higher insertion loss is achieved even with the tuning screws present in the filter. This could be corrected if the radii of side resonators are included in the simulation and the design is optimised before manufacturing the filters.

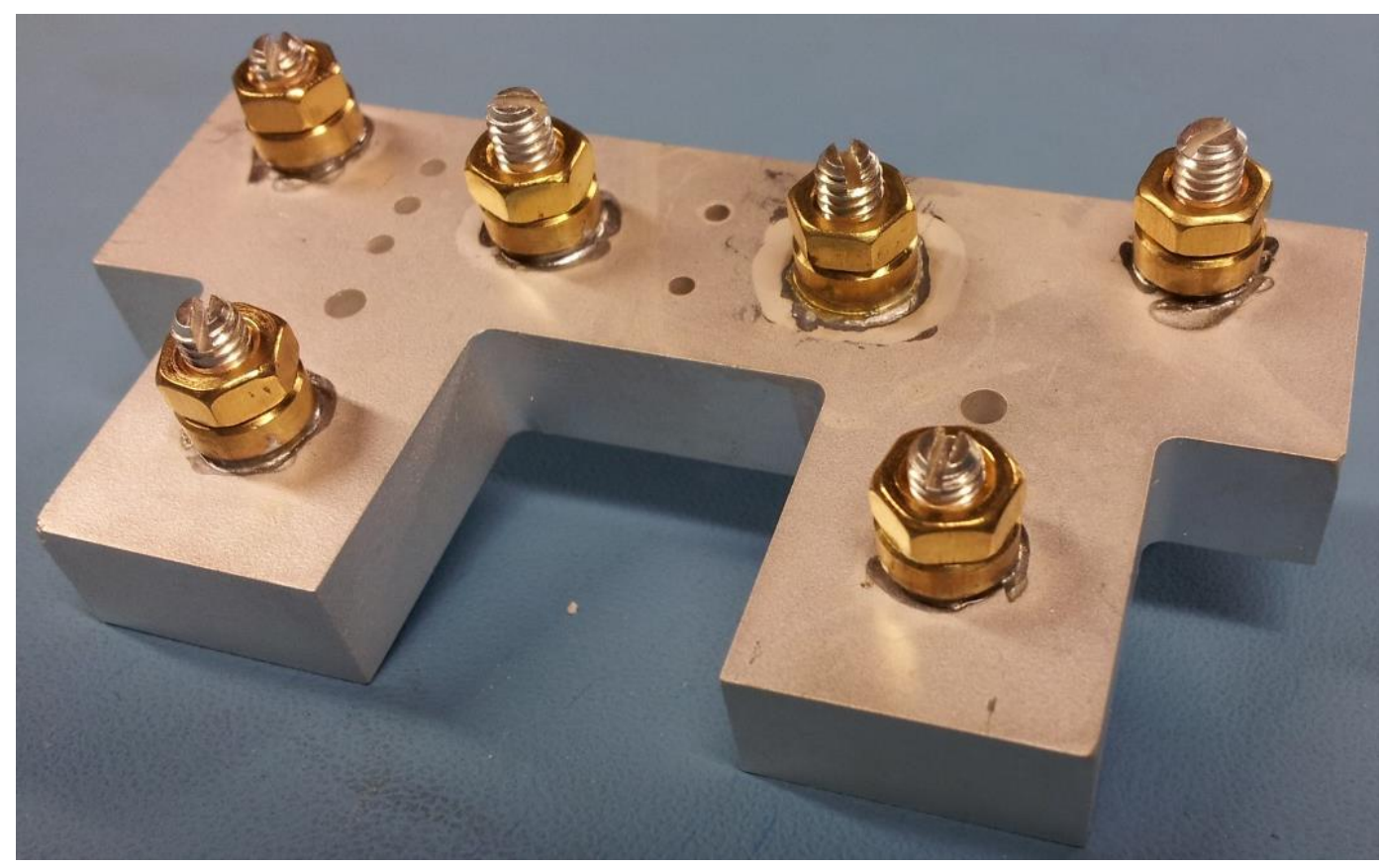

Figure 5.28 : Fabricated generalised Chebyshev filter with tuning screws 


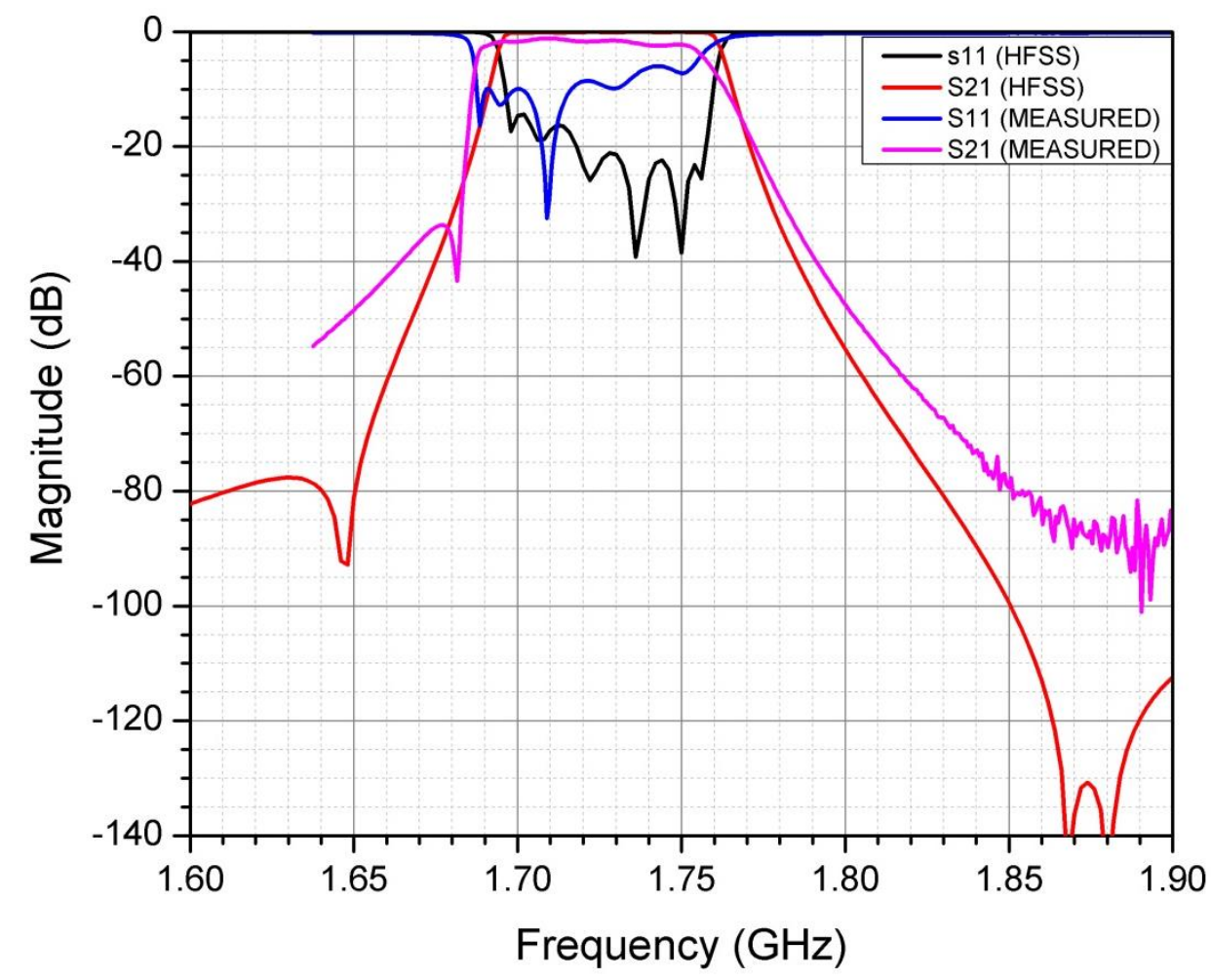

Figure 5.29 : Simulated and measured response of generalised Chebyshev filter with tuning screws

\subsection{Summary}

A generalized Chebyshev ceramic waveguide filter with transmission zeros at above and below the pass band is designed. Inductive and capacitive cross coupled triplets are designed to realize transmission zeros at both sides of the pass band. Inductive cross coupling is achieved by through holes in the ceramic waveguide between resonator 1 and 3. Capacitive cross coupling in ceramic waveguide is realized using silver coated blind hole between resonator 4 and 6 of generalized Chebyshev ceramic waveguide filter. The blind hole placed at the middle of the broad wall of the ceramic waveguide filter acts as a resonating coupling structure and therefore another transmission zero is produced in the filter response due to resonant coupling structure. The position of this extra attenuation pole can be controlled by varying the dimension of the blind hole. Another generalised Chebyshev filter is designed with tuning screws in it to tune the resonances

of individual resonators. 


\section{INTEGRATED CERAMIC}

WAVEGUIDE DIPLEXER

\subsection{Introduction}

Diplexers are essential component of RF front end of cellular base station which isolate sensitive receive section from the transmit section while sharing the common antenna port. It is a three port device which consists of transmit and receive filter branches often connected in parallel through a common junction. Diplexers can be physically realized in various physical structures such as microstrip line, coaxial resonator or waveguide dictated by the electrical performance, operating frequency, mechanical constraints and filter technology. Diplexers are essentially two channel multiplexers and can be accomplished by designing individual doubly terminated band pass filters for each branch and then connecting them in parallel [20]. However, interaction between two filters must be avoided by optimizing the common junction. In a waveguide diplexer the realization of the common junction consists of a magic-T or $\mathrm{Y}$-junction. A T-junction is a lossless reciprocal three port network. A small tuning of input coupling and first resonator is required on each filter branch. Although there exist some exact synthesis methods to design a diplexer in the literature [72-75]. Yet the most common approach used to design a microwave diplexer is based on the optimization techniques [76, 77]. If the separation between RX and TX band filters is wide enough then optimisation techniques can give satisfactory results in a very small time. The diplexer can be divided into two major sections, the waveguide channel filters and common junction. 
A ceramic rectangular waveguide diplexer is designed with a Chebyshev filter in its transmit branch and a generalized Chebyshev filter for receive path. Generalized Chebyshev filter is used to design RX section of the diplexer while TX branch consists of Chebyshev filter. Each filter section and common junction can be designed separately and then combined in circuit simulator for fast optimization process [76]. A general diplexer configuration in a mobile base station is shown in Figure 1.1

\subsection{G diplexer specifications}

A ceramic waveguide diplexer design is explained in this Chapter based on the specifications given in Table 6-1. The ceramic material used to design the waveguide diplexer is Barium Titanate $\left(\mathrm{BaTiO}_{3}\right)$ with relative permittivity 43 and loss tangent of 0.00004 . Metal coating of silver ink with conductivity $4.4 \times 10^{7} \Omega^{-1} \mathrm{~m}^{-1}$ is used.

Table 6-1: Diplexer specification

\begin{tabular}{|l|l|}
\hline \multicolumn{2}{|c|}{ Transmit Path } \\
\hline Passband Bandwidth & $60 \mathrm{MHz}(2100 \mathrm{MHz}-2160 \mathrm{MHz})$ \\
\hline Passband Centre Frequency & $2130 \mathrm{MHz}$ \\
\hline Maximum Insertion Loss & $<0.7 \mathrm{~dB}$ \\
\hline Passband Return Loss & $>20 \mathrm{~dB}$ \\
\hline Stopband Attenuation & $>50 \mathrm{dBc}$ at f<2060 MHz \& f $>2200 \mathrm{MHz}$ \\
\hline & Receive Path \\
\hline Centre frequency & $1730 \mathrm{MHz}$ \\
\hline Passband Bandwidth & $60 \mathrm{MHz}$ \\
\hline Maximum Insertion Loss & $<0.7 \mathrm{~dB}$ \\
\hline Passband Return Loss & $>20 \mathrm{~dB}$ \\
\hline Stopband Attenuation & $\geq 70 \mathrm{~dB}$ at $\mathrm{DC}<\mathrm{f}<1650 \mathrm{MHz}$ \\
& $\geq 80 \mathrm{~dB}$ at $1880 \mathrm{MHz}$ f $<2200 \mathrm{MHz}$ \\
\hline
\end{tabular}

\subsection{Transmit channel ceramic filter}

A six pole Chebyshev ceramic waveguide filter is designed to be used for transmit path 
of the diplexer. The pass band extends from $2.1 \mathrm{GHz}$ to $2.16 \mathrm{GHz}$ with out of band rejection level meeting the specifications given in Table 6-1.

\subsubsection{Lumped element design}

The ripple level in the passband of the Chebyshev filter for return loss $L_{R}=20 \mathrm{~dB}$ is calculated using Equation 2.19 as

$$
\varepsilon==0.1005 \mathrm{~dB}
$$

The order of the filter required to meet the out of band attenuation specification can be calculated using Equation2.23

$$
N \geq 5.86
$$

The coupling coefficients of the inverter coupled Chebyshev low pass prototype are calculated using Equation 2.24 and Equation 2.26 as

$K_{01}=K_{6 o u t}=1$

$K_{12}=K_{56}=1.3875$

$K_{23}=K_{45}=1.9431$

$K_{34}=2.1681$

And the shunt capacitive elements of a Chebyshev inverter coupled lowpass prototype are evaluated using Equation 2.25 as,

$C_{1}=C_{6}=0.9958$

$C_{2}=C_{5}=2.7205$

$C_{3}=C_{4}=3.7163$

Figure 6.1 represents a normalised Chebyshev inverter coupled low pass prototype filter network. For a centre frequency of $2.130 \mathrm{GHz}$ and $\mathrm{z}=50 \Omega$,

$$
\omega=2 \pi f_{0}=13.38 \times 10^{9}
$$

And 


$$
\begin{gathered}
\nabla=\frac{f_{0}}{B w} \\
=\frac{2130 \times 10^{6}}{60 X 10^{6}}=35.5
\end{gathered}
$$

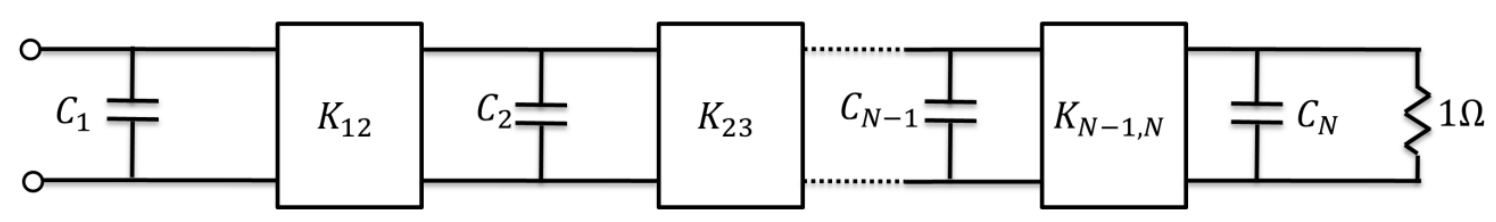

Figure 6.1: Capacitive coupled normalised low pass prototype filter

The element values of a lowpass to bandpass frequency and impedance scaled capacitive coupled network shown in Figure 6.2 become,

$$
\begin{gathered}
C_{01}=C_{N . N+1}=\frac{1}{\omega z(\nabla-1)^{\frac{1}{2}}} \\
C_{01}=C_{6 O U T}=0.254 p F
\end{gathered}
$$

And

$$
C_{r . r+1}=\frac{K_{r . r+1}}{z \nabla \omega} \quad(r=1,2 \ldots N-1)
$$

Thus,

$$
\begin{aligned}
& C_{12}=C_{56}=0.0584 p F \\
& C_{23}=C_{45}=0.0818 p F \\
& C_{34}=0.0912 p F
\end{aligned}
$$

And the shunt resonator element values become [3],

$$
L_{r . r}=\frac{z}{C_{r} \omega}
$$

This gives,

$$
L_{11}=L_{66}=3.752 n H
$$


$L_{22}=L_{55}=1.373 n H$

$L_{33}=L_{44}=1.005 \mathrm{nH}$

And

$$
\begin{gathered}
C_{11}=\frac{\left[\frac{C_{1}}{\omega}-\frac{(\nabla-1)^{\frac{1}{2}}}{\omega \nabla}-C_{12}\right]}{Z} \\
=1.1824 \mathrm{pF}
\end{gathered}
$$

And

$$
\begin{aligned}
& C_{N N}= \frac{\left[\frac{C_{N}}{\omega}-\frac{(\nabla-1)^{\frac{1}{2}}}{\omega \nabla}-C_{N-1 . N}\right]}{Z} \\
&=c 66=1.1824 p F
\end{aligned}
$$

And

$$
C_{r r}=\frac{\left[\frac{C_{r}}{\omega}-\frac{(\nabla-1)^{\frac{1}{2}}}{\omega \nabla}-C_{r-1 . r}-C_{r . r+1}\right]}{Z}
$$

Thus

$C_{22}=3.925 p F$

$C_{33}=5.381 p F$

$C_{44}=5.381 p F$

$C_{55}=3.925 p F$

A circuit simulation of six section Chebyshev filter shown in the Figure 6.2 is given in Figure 6.3. The insertion loss in the passband of the filter is less than $0.7 \mathrm{~dB}$ for resonators with unloaded Q-factor of 2400. 


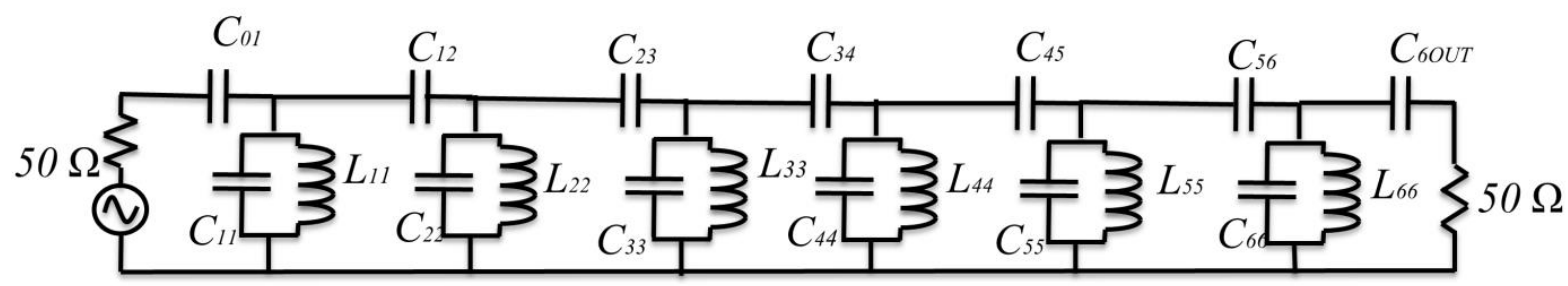

Figure 6.2: Capacitive coupled Chebyshev bandpass filter circuit diagram

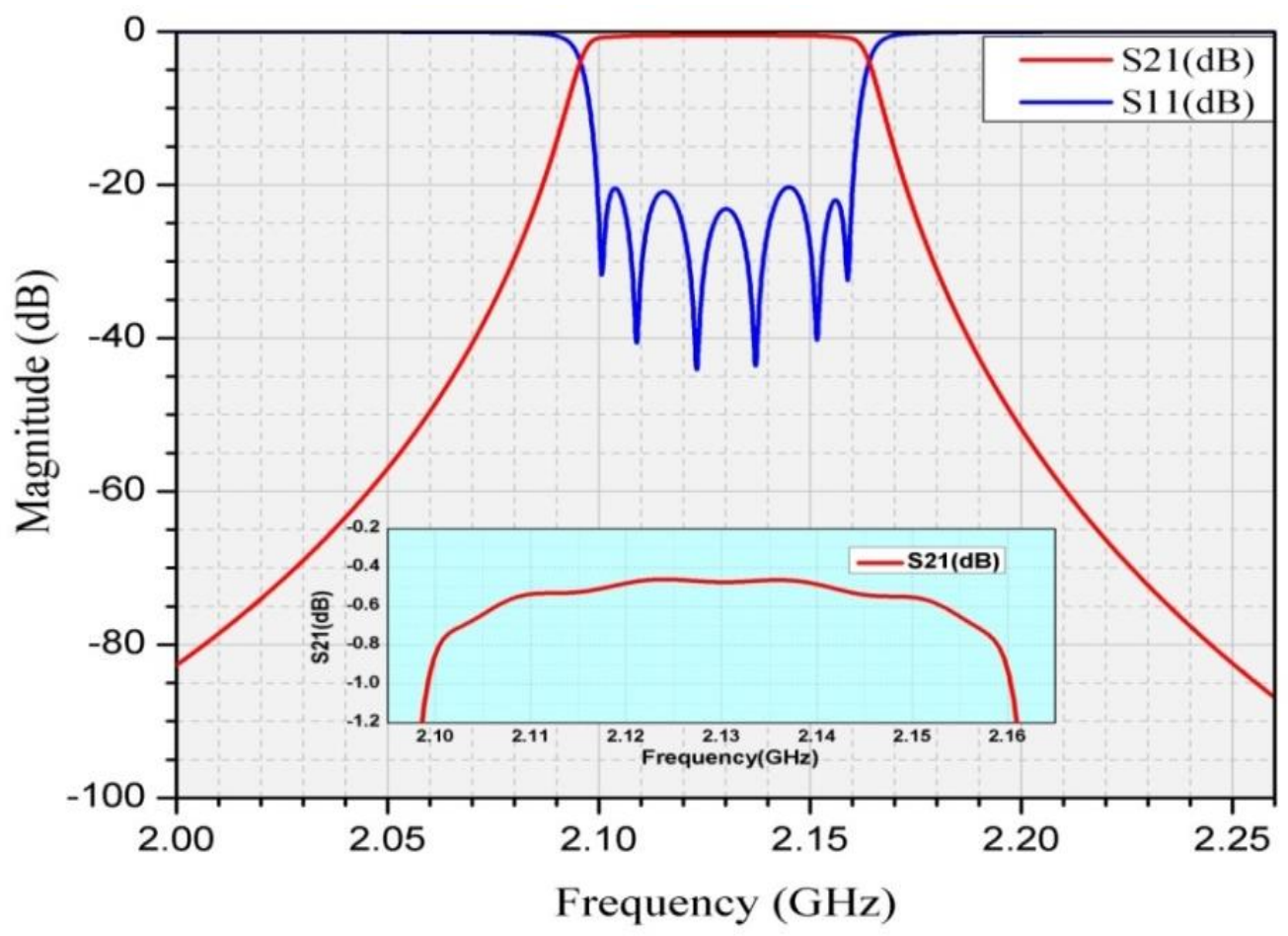

Figure 6.3: Lumped element Chebyshev bandpass filter response (Circuit simulation)

\subsubsection{Ceramic waveguide TX filter}

The dimensions of the ceramic waveguide resonator are calculated using Equation 3.1 for a resonance of $2130 \mathrm{MHz}$. As the goal is to design a monolithic integrated ceramic waveguide diplexer, therefore the broad dimension and height of the resonators to be used in TX filter is maintained same as for RX filter for simplicity. For RX filter with centre frequency of $1.730 \mathrm{GHz}$ designed in Chapter.5, $\mathrm{a}=\mathrm{d}=18.64 \mathrm{~mm}$ and $\mathrm{b}=$ $9.32 \mathrm{~mm}$, thus only length (d) of the resonators are needed to be adjusted to produce a fundamental frequency of $2.130 \mathrm{GHz}$. 
For a rectangular ceramic waveguide resonator with an aspect ratio $\frac{b}{a}=0.5$, by putting $a=18.64 \mathrm{~mm}$ and $f_{0}=2130 \mathrm{MHz}$ and $\varepsilon_{r}=43$ in Equation 3.1, we get

$$
d=13.1 \mathrm{~mm}
$$

Therefore the silver plated ceramic waveguide resonator with an aspect ratio of 0.5 has the dimensions of $18.64 \times 13.1 \times 9.32(\mathrm{~mm})$ to resonate at a fundamental frequency of $2130 \mathrm{MHz}$. The calculated unloaded Q-factor for the $\mathrm{TE}_{10}$ mode of silver coated rectangular ceramic waveguide resonator, at a centre frequency of $2130 \mathrm{MHz}$, using Equation 4.21and Equation 4.23 is calculated as

$$
Q_{u}=2630
$$

By including the effect of dielectric loss tangent of the material, i.e. $\tan \delta=0.00004$. The total unloaded Q-factor becomes using Equation 4.24

$$
Q_{\text {total }}=2599
$$

Table 6-2 shows the fundamental resonance frequency, first spurious resonance and the Q-factor values of the ceramic waveguide resonator obtained from $\mathrm{HFSS}^{\mathrm{TM}}$ simulation. It is observed that the first spurious resonance occurs at $2740 \mathrm{MHz}$, consequently providing a wide spurious free stopband bandwidth.

Table 6-2: Ceramic waveguide resonator eigenmode data $\left(\mathrm{HFSS}^{\mathrm{TM}}\right)$

\begin{tabular}{|c|c|c|}
\hline Mode Number & Frequency (MHz) & Q-Factor \\
\hline 01 & 2132 & 2370 \\
\hline 02 & 2740 & 2460 \\
\hline
\end{tabular}

Inter resonator couplings are achieved via silver coated through holes acting as impedance inverters placed symmetrically in the broad wall of waveguide. The amount of coupling achieved is a function of radius of hole and its distance from the side walls [78]. Figure 6.4 shows the coupling bandwidth achieved as a function of distance of hole from side walls with symmetrically placed fixed diameter holes in the broad wall of ceramic rectangular waveguide. 


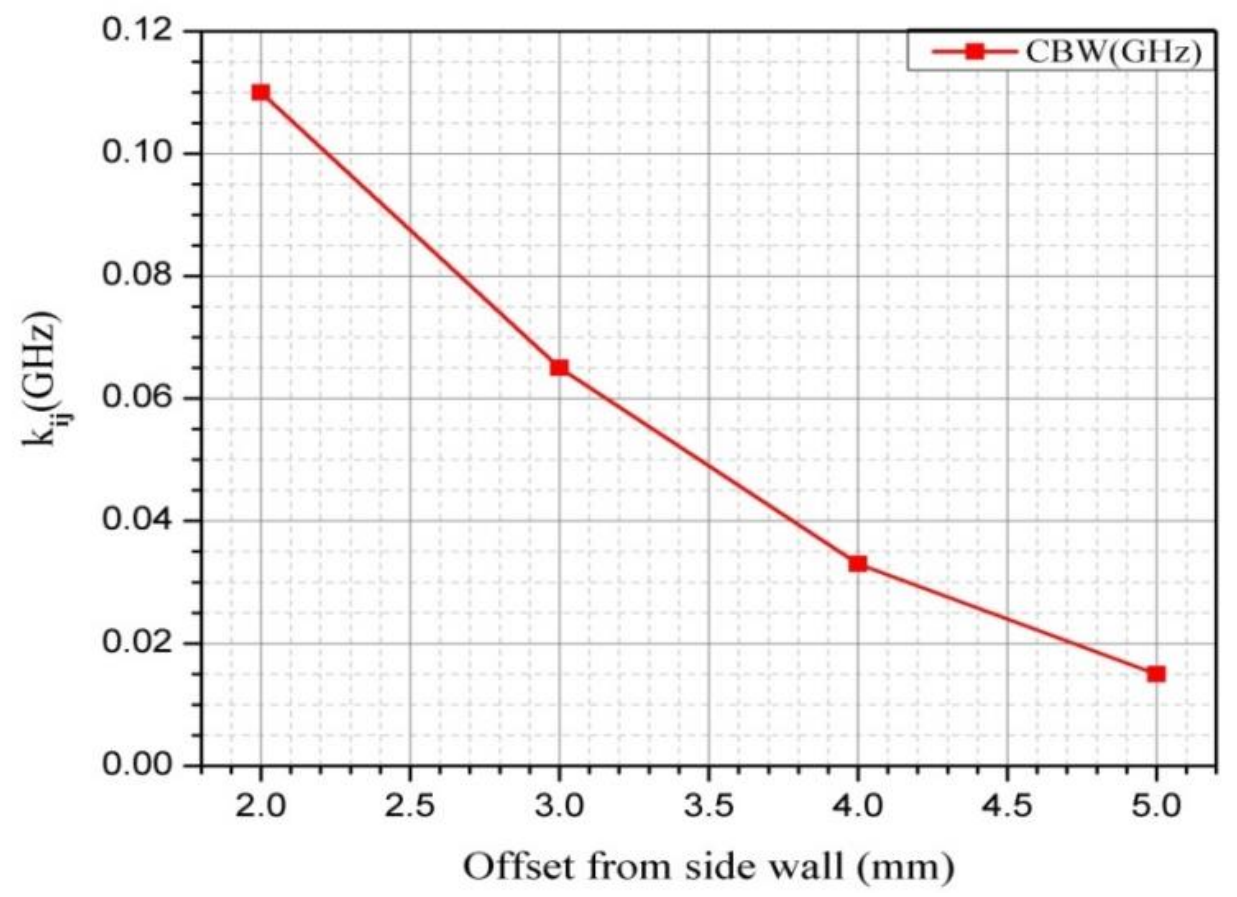

Figure 6.4: Inter resonator coupling bandwidths variation with respect to hole offset $\left(\mathrm{X}_{1}\right)$ from side walls of the waveguide

The external coupling to and from filter is achieved via coaxial probe placed at the centre of first and last resonator. Figure 6.5 and Figure 6.6 show the final TX filter layout and its S-parameter response, respectively.

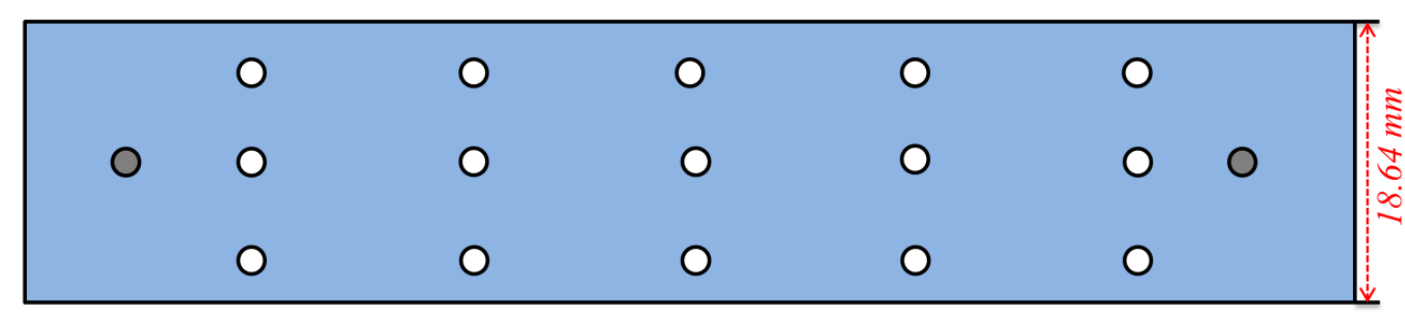

(i)

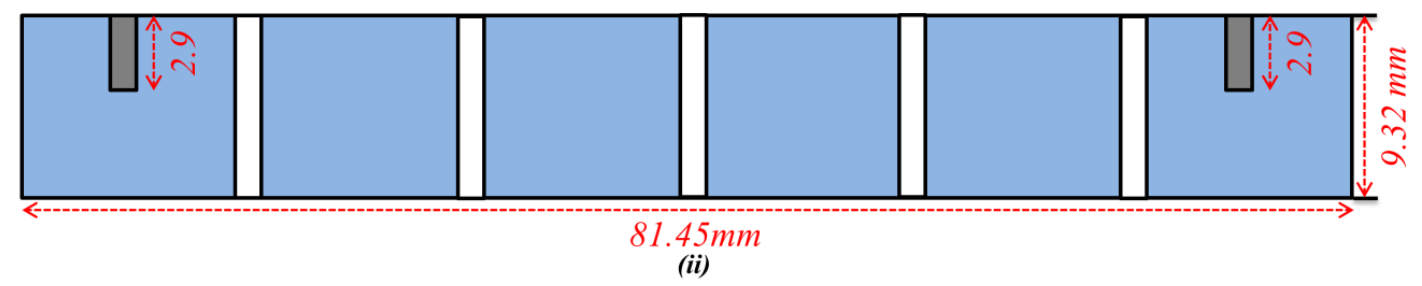

Figure 6.5: TX Chebyshev ceramic waveguide filter layout (i) Top view (ii) Side view 


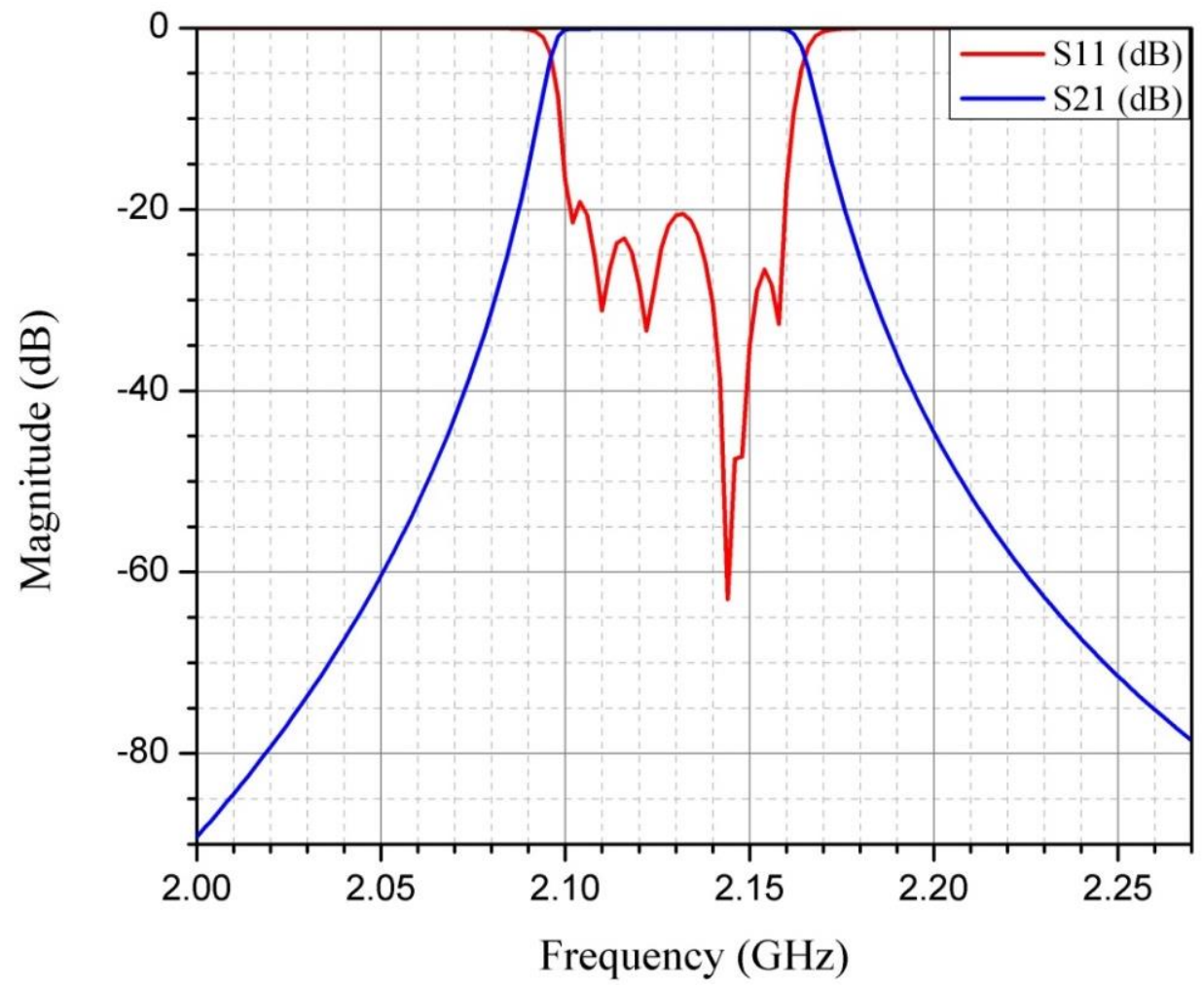

Figure 6.6: Simulated TX filter response $\left(\mathrm{HFSS}^{\mathrm{TM}}\right)$

The EM simulated result shows a $20 \mathrm{~dB}$ return loss in the passband of the six section Chebyshev ceramic rectangular waveguide filter with $60 \mathrm{MHz}$ bandwidth. All inter resonator coupling are realized using metal plated through holes in the waveguide placed at half guide wavelength periods from each other.

\subsection{Receive channel ceramic filter}

A six pole generalized Chebyshev ceramic waveguide filter is designed for receive filter path. The pass band extends from $1.7 \mathrm{GHz}$ to $1.76 \mathrm{GHz}$ with out of band rejection level meeting the specifications given in Table 6-1.

\subsubsection{Circuit design}

A six section generalized Chebyshev filter is designed using coupling matrix extracted from [22] as described in Chapter 5. The filter comprising two cross coupled triplets to realize the transmission zeros at both sides of the passband to meet the required rejection level in the stop band. Figure 6.7 shows the coupling matrix for the 
generalised Chebyshev filter with two cross couplings.

$\left[\begin{array}{ccccccccc} & S & 1 & 2 & 3 & 4 & 5 & 6 & L \\ S & 0 & 1.0626 & 0 & 0 & 0 & 0 & 0 & 0 \\ 1 & 1.0626 & -0.0035 & 0.8897 & 0.1218 & 0 & 0 & 0 & 0 \\ 2 & 0 & 0.8897 & -0.1749 & 0.6232 & 0 & 0 & 0 & 0 \\ 3 & 0 & 0.1218 & 0.6232 & 0.0101 & 0.5993 & 0 & 0 & 0 \\ 4 & 0 & 0 & 0 & 0.5993 & -0.0341 & 0.6097 & -0.1969 & 0 \\ 5 & 0 & 0 & 0 & 0 & 0.6097 & 0.2713 & 0.8761 & 0 \\ 6 & 0 & 0 & 0 & 0 & -0.1969 & 0.8761 & -0.0035 & 1.0626 \\ L & 0 & 0 & 0 & 0 & 0 & 0 & 1.0626 & 0\end{array}\right]_{\text {? }}$

Figure 6.7: Coupling matrix for Receive channel filter

The element values of the lumped element resonator and impedance inverter are already calculated in Chapter 5. Figure 5.3 shows the final layout of inverter coupled lumped element generalised Chebyshev band pass filter for receive channel and its circuit simulated response is given in Figure 6.8.

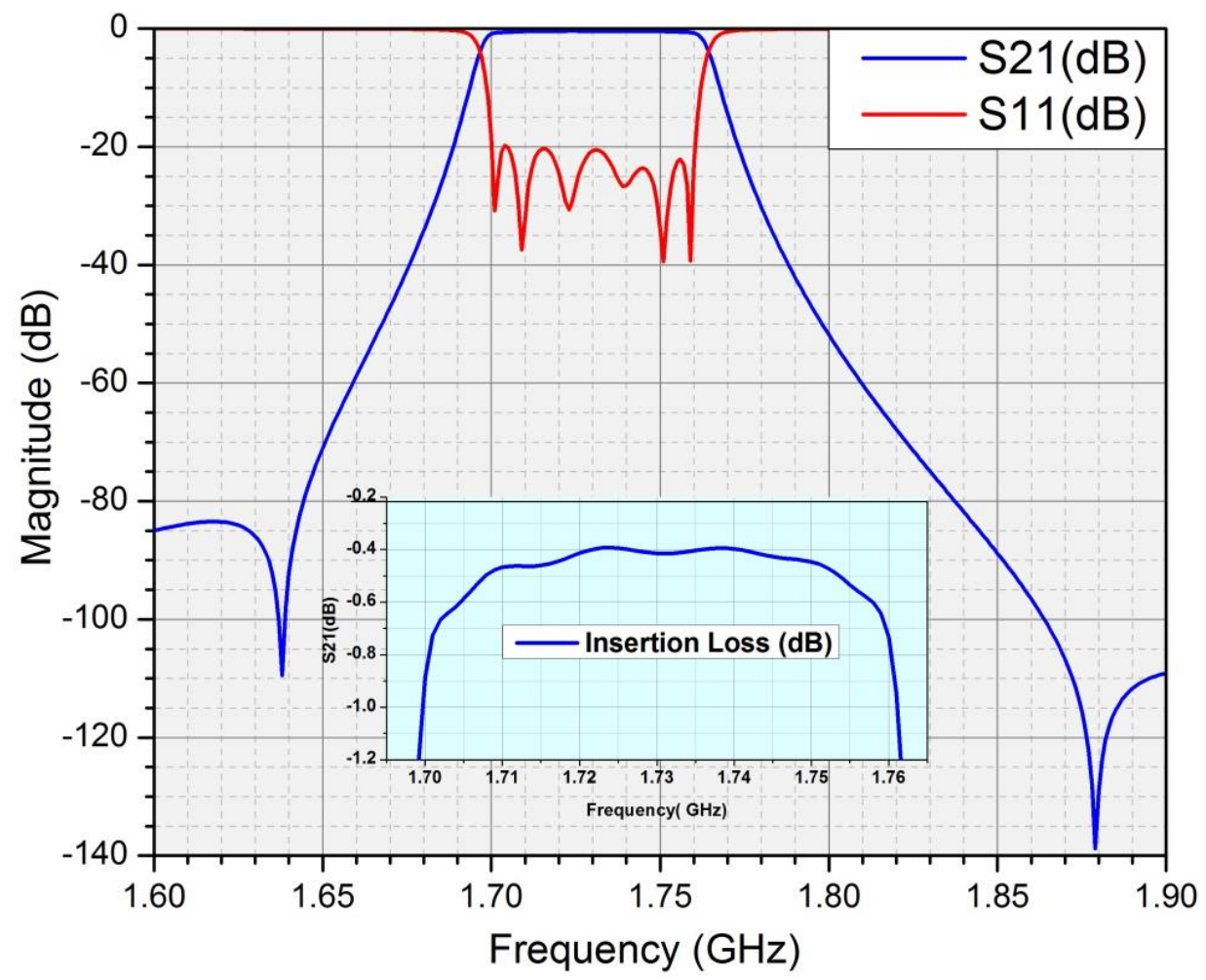

Figure 6.8: Simulated RX filter circuit design response 


\subsubsection{Ceramic waveguide realisation}

A silver plated ceramic $\left(\varepsilon_{r}=43\right)$ waveguide resonator with an aspect ratio of 0.5 has the dimensions of $18.64 \times 18.64 \times 9.32(\mathrm{~mm})$ to resonate at a fundamental frequency of 1732 MHz. Table 6-3 shows the fundamental resonance frequency, first spurious resonance and the Q-factor values of the ceramic waveguide resonator obtained from HFSS $^{\mathrm{TM}}$ simulation. It is observed that the first spurious resonance occurs at $2740 \mathrm{MHz}$, thus, providing a wide spurious free stopband bandwidth.

Table 6-3 : Ceramic WG resonator eigenmode data $\left(\mathrm{HFSS}^{\mathrm{TM}}\right)$

\begin{tabular}{|c|c|c|}
\hline Mode Number & Frequency (MHz) & Q-Factor \\
\hline 01 & 1732 & 2430 \\
\hline 02 & 2740 & 2700 \\
\hline
\end{tabular}

The length $(d)$ of the each resonator used in the filter can be modified slightly according to the given coupling matrix. Table 6-4 summarises the modified lengths of each resonator according to the self-coupling values given in the coupling matrix.

Table 6-4 : Modified resonator lengths based on loaded resonance values

\begin{tabular}{|c|c|c|c|}
\hline Resonator & $\begin{array}{c}\text { Self-coupling } \\
\text { coefficient }\end{array}$ & $\begin{array}{c}\text { Frequency } \\
(\mathrm{MHz})\end{array}$ & $\begin{array}{c}\text { Resonator length }(d) \\
\text { (centre pin to centre pin) }\end{array}$ \\
\hline 01 & -0.00012 & 1731 & $18.70 \mathrm{~mm}$ \\
\hline 02 & -0.00606 & 1738 & $18.54 \mathrm{~mm}$ \\
\hline 03 & 0.00035 & 1732 & $18.64 \mathrm{~mm}$ \\
\hline 04 & -0.00118 & 1734 & $18.60 \mathrm{~mm}$ \\
\hline 05 & 0.0094 & 1723 & $18.87 \mathrm{~mm}$ \\
\hline 06 & -0.00012 & 1734 & $18.60 \mathrm{~mm}$ \\
\hline
\end{tabular}


Inter resonator direct couplings are achieved via silver coated through holes acting as impedance inverters placed symmetrically in the broad wall of the waveguide. In ceramic waveguide filter all of the inductive couplings are realized via silver coated through holes. The capacitive coupling is achieved via silver coated blind hole. The amount of coupling achieved is dependent on the radius and depth of hole inside the waveguide. The external coupling to and from filter is achieved via coaxial probe placed at the centre of first and last resonator. The coupling scheme of the six pole generalised Chebyshev filter used for receive channel of the diplexer is shown in the Figure 5.19.

Figure 6.9 shows the final layout of the ceramic waveguide generalised Chebyshev bandpass filter for RX branch of diplexer. The HFSS ${ }^{\mathrm{TM}}$ simulated response of the filter is shown in the Figure 6.10.

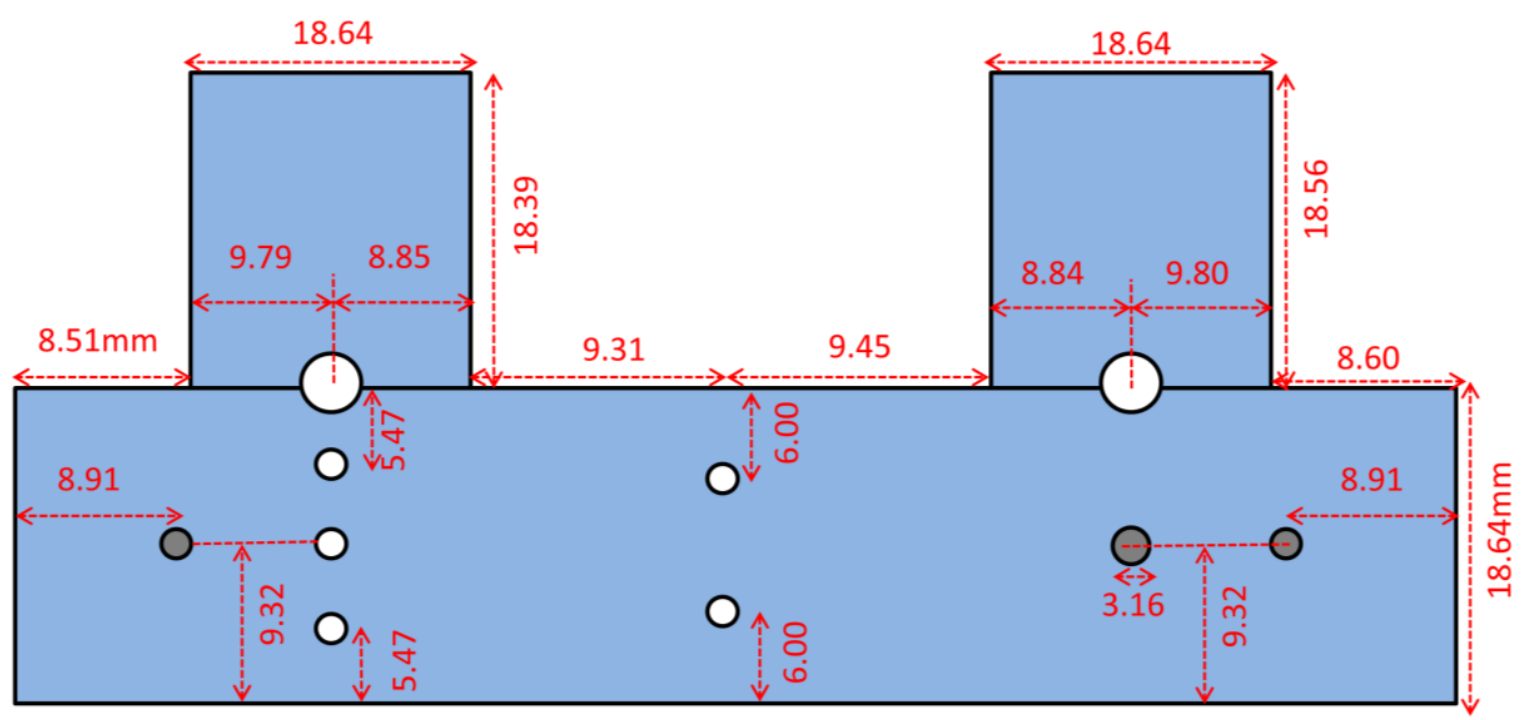

(i) Top View

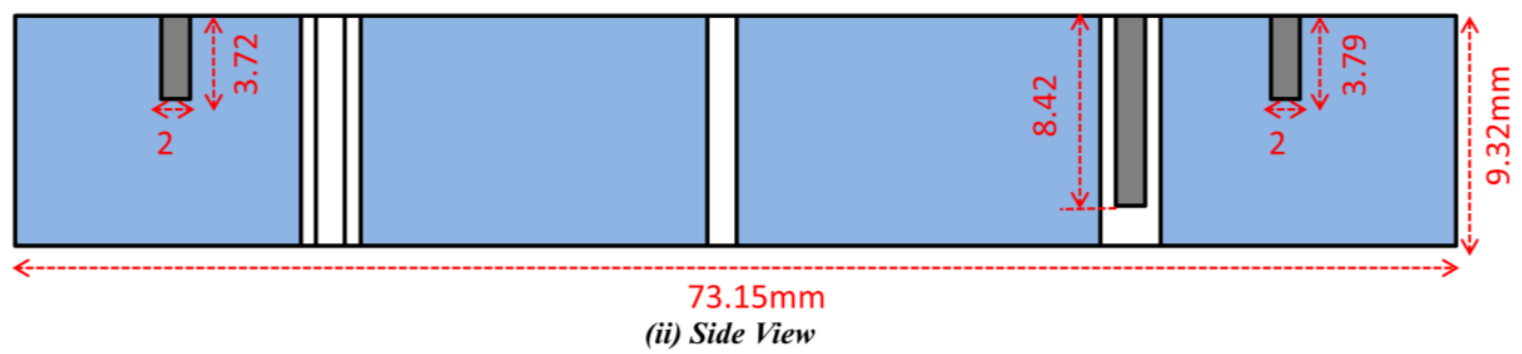

Figure 6.9: Generalized Chebyshev RX ceramic waveguide filter layout 


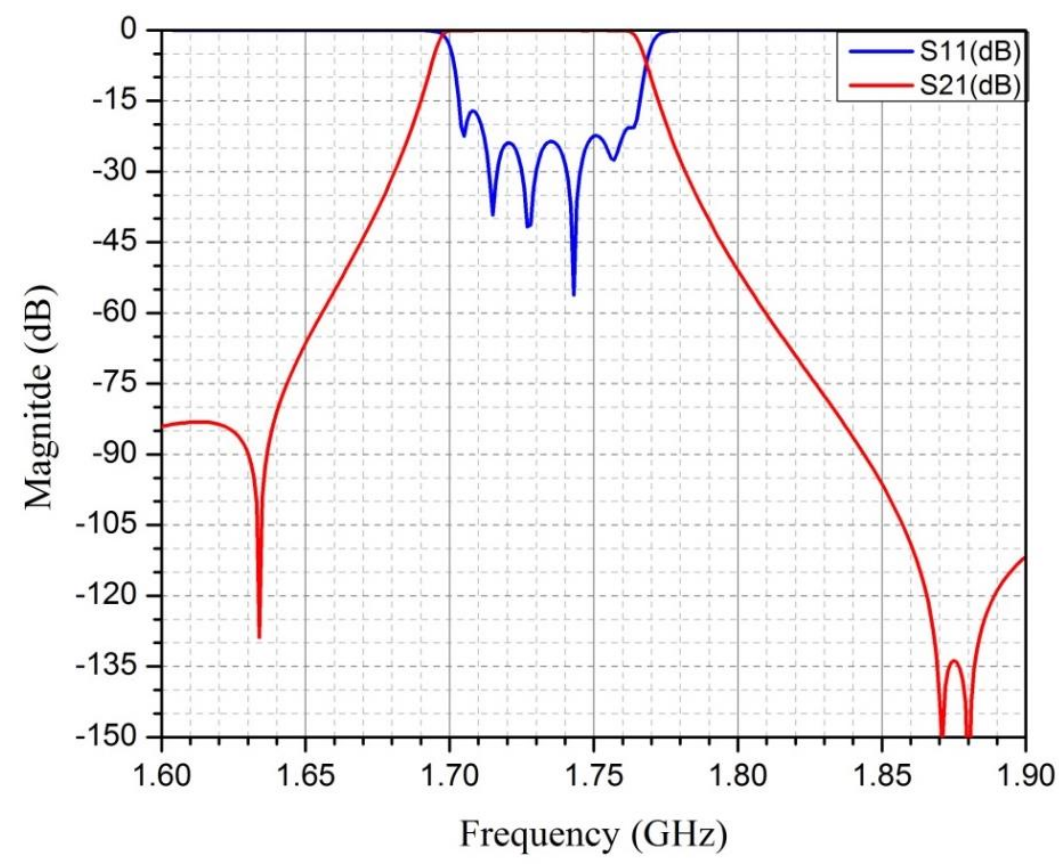

Figure 6.10: Simulated generalized Chebyshev RX filter response (HFSS ${ }^{\mathrm{TM}}$ )

Figure 6.11 shows the detailed passband insertion loss of the generalised Chebyshev bandpass filter.

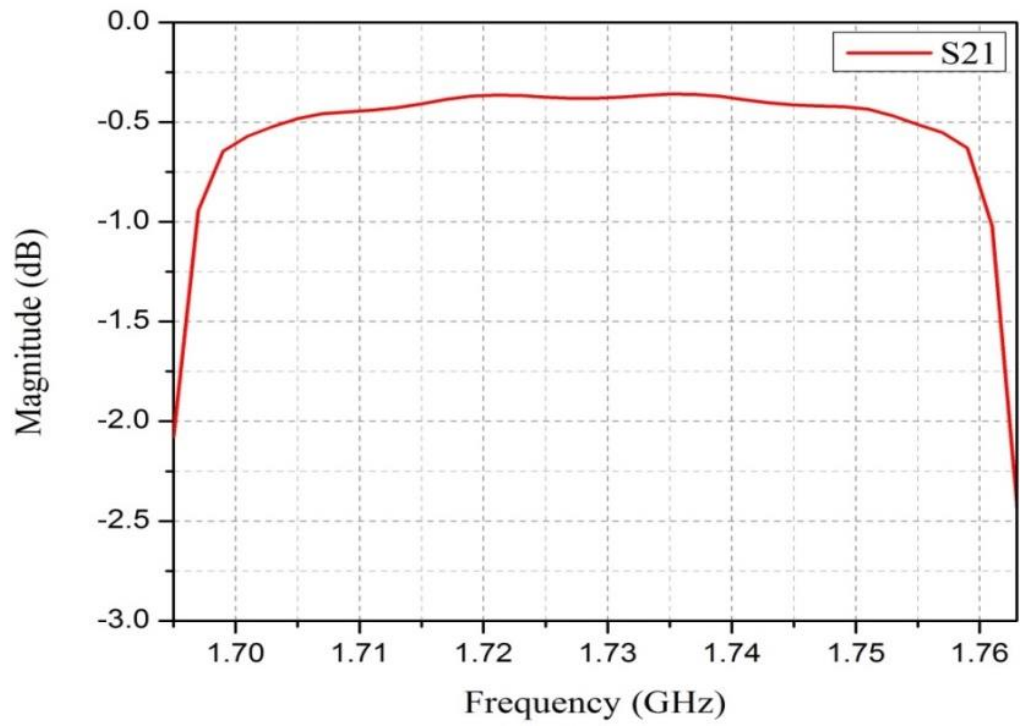

Figure 6.11: Generalized Chebyshev RX filter pass band insertion loss

\subsection{Diplexer design}

A diplexer can be designed by placing the two doubly terminated bandpass filters parallel to each other. Due to the loading effect, the first resonator and the input 
coupling bandwidths need to be slightly modified. If the centre frequencies of both filters are not too close then only a small amount of tuning is needed. Figure 6.12 represents the block diagram of the diplexer combining a Chebyshev (TX) bandpass filter and a generalized Chebyshev (RX) bandpass filter. Both filter branches share the common antenna at the common port simultaneously.

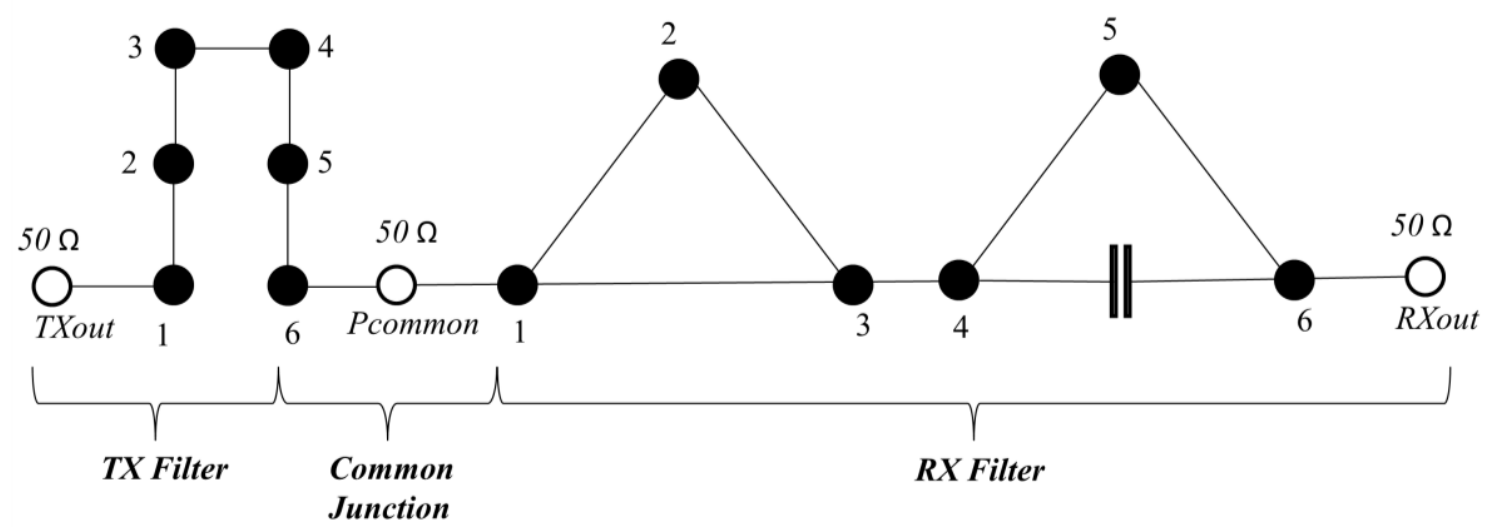

Figure 6.12: Diplexer configuration

\subsubsection{Circuit design}

The transmit and receive filters designed in section 6.3 and 6.4 are combined to form a diplexer. Table 6-5 summarises the various coupling coefficients for the circuit design of individual filter branches and diplexer shown in Figure 6.13. Each ' $\mathrm{K}$ ' block in the Figure 6.13 shows the impedance inverter between resonators as explained in Figure 5.2. It is observed that a slight modification in input coupling coefficient and resonant frequency of first resonator section in each filter branch is needed. The coupling coefficients shown here are the coupling bandwidth of the inverters in $\mathrm{GHz}$ for individual filters and for diplexer.

Table 6-5: Coupling coefficients of individual filter branches and of diplexer

\begin{tabular}{|c|l|l|}
\hline \multicolumn{3}{|c|}{ RX FILTER } \\
\hline Coupling Coefficient $(\mathrm{GHz})$ & \multicolumn{1}{|c|}{ Filter } & \multicolumn{1}{c|}{ Diplexer } \\
\hline$K_{\text {Rin } 1}$ & 0.06432 & 0.06237 \\
\hline$K_{R 12}$ & 0.05087 & 0.05000 \\
\hline$K_{R 23}$ & 0.03634 & 0.03634 \\
\hline
\end{tabular}




\begin{tabular}{|c|c|c|}
\hline$K_{R 34}$ & 0.03543 & 0.03543 \\
\hline$K_{R 45}$ & 0.03558 & 0.03558 \\
\hline$K_{R 56}$ & 0.05070 & \begin{tabular}{|l|}
0.05070 \\
\end{tabular} \\
\hline$K_{R 60 U T}$ & 0.06237 & \begin{tabular}{|l|}
0.06200 \\
\end{tabular} \\
\hline$K_{R 13}$ & 0.006173 & \begin{tabular}{|l|}
0.006173 \\
\end{tabular} \\
\hline$K_{R 46}$ & -0.01093 & -0.01093 \\
\hline$f_{R 1}$ & 1.731 & 1.73 \\
\hline$f_{R 2}$ & 1.735 & 1.735 \\
\hline$f_{R 3}$ & 1.73 & 1.73 \\
\hline$f_{R 4}$ & 1.732 & 1.732 \\
\hline$f_{R 5}$ & 1.723 & 1.723 \\
\hline$f_{R 6}$ & 1.732 & 1.732 \\
\hline \multicolumn{3}{|c|}{ TX FILTER } \\
\hline$K_{\operatorname{Tin} 1}$ & 0.06311 & 0.06277 \\
\hline$K_{T 12}$ & 0.05158 & 0.05158 \\
\hline$K_{T 23}$ & 0.03726 & 0.03726 \\
\hline$K_{T 34}$ & 0.03524 & 0.03524 \\
\hline$K_{T 45}$ & 0.03726 & 0.03726 \\
\hline$K_{T 56}$ & 0.05158 & 0.05158 \\
\hline$K_{T 6 O U T}$ & 0.06277 & \begin{tabular}{|l|}
0.06277 \\
\end{tabular} \\
\hline$f_{R 1}$ & 2.13 & 2.131 \\
\hline$f_{R 2}$ & 2.13 & 2.13 \\
\hline$f_{R 3}$ & 2.13 & 2.13 \\
\hline$f_{R 4}$ & 2.13 & 2.13 \\
\hline$f_{R 5}$ & 2.13 & 2.13 \\
\hline$f_{R 6}$ & 2.13 & 2.13 \\
\hline
\end{tabular}

The inverter coupled lumped element diplexer diagram is shown in the Figure 6.13 and its circuit simulation response is shown in the Figure 6.14. As there exists a guard band of $400 \mathrm{MHz}$ between TX and RX filters of diplexer, therefore, only a small tuning is required to get the diplexer response when doubly terminated bandpass filters are 
connected together.

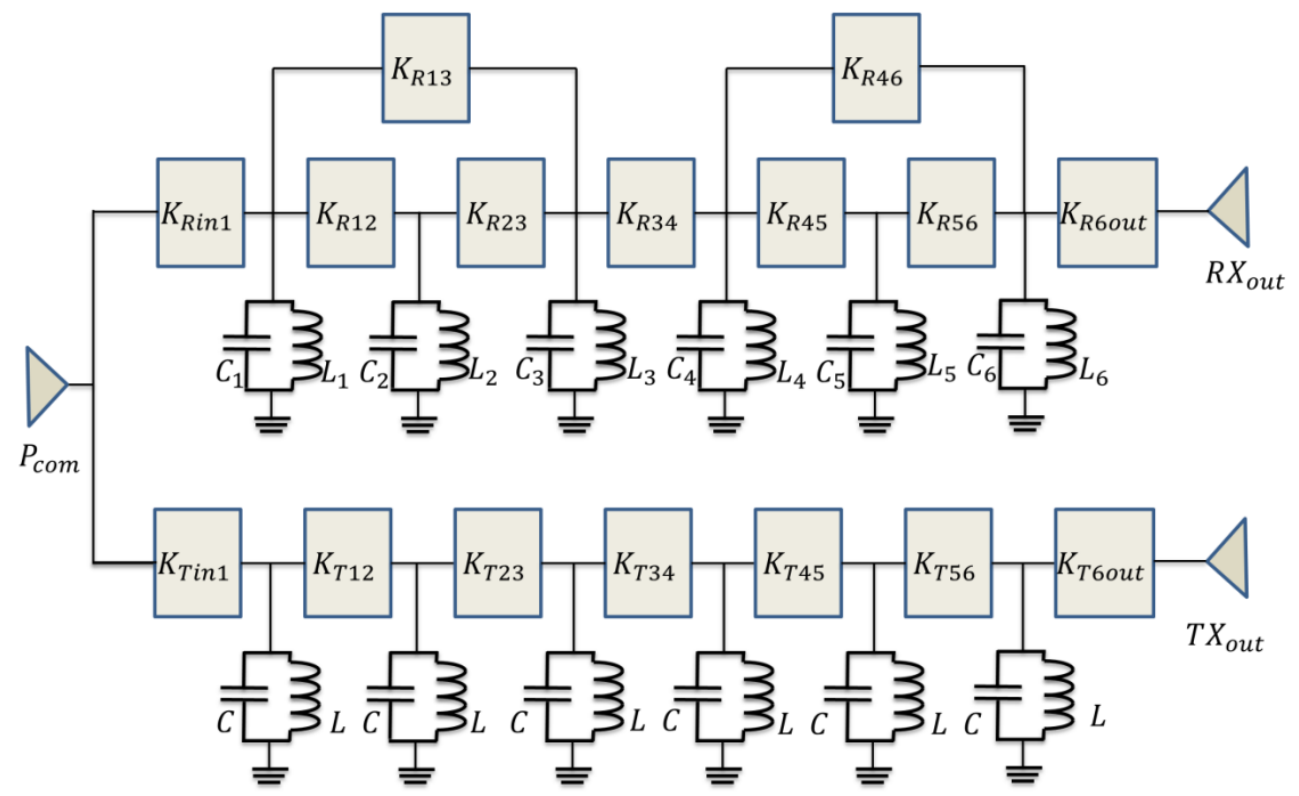

Figure 6.13: Inverter-coupled diplexer circuit design

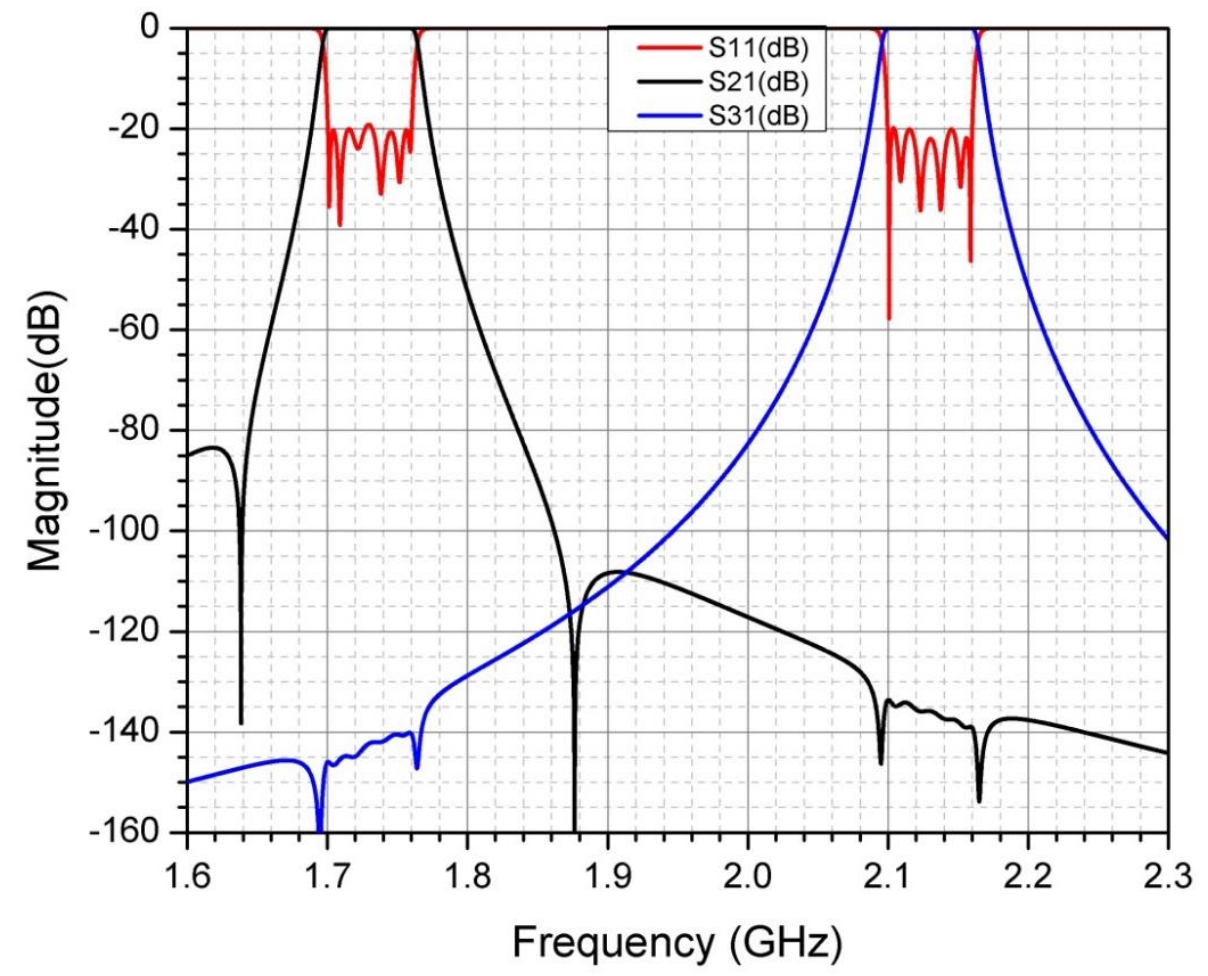

Figure 6.14: Simulated diplexer circuit design response 

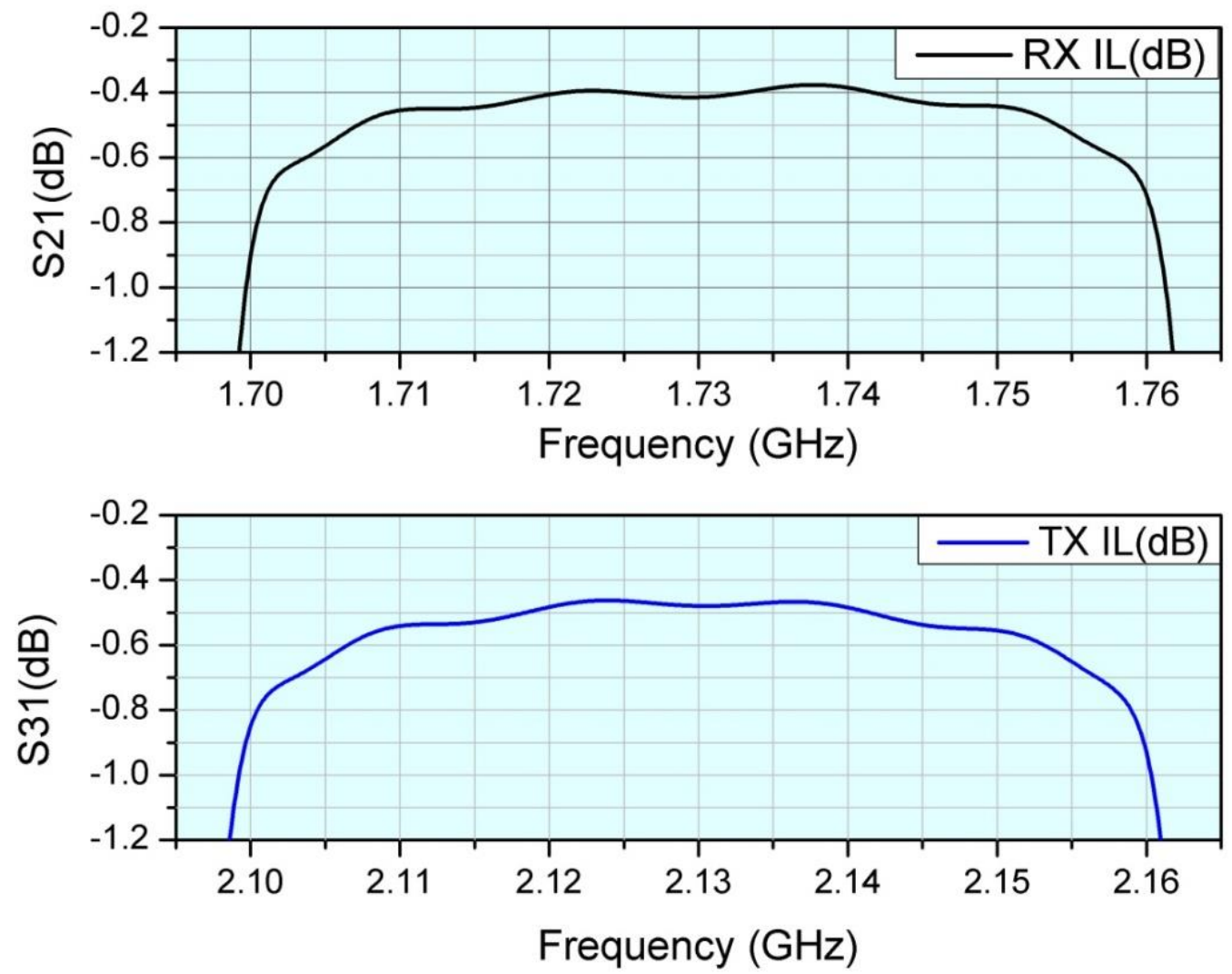

Figure 6.15: Passband insertion loss of RX and TX filter

\subsubsection{Ceramic waveguide realisation of diplexer}

The ceramic waveguide diplexer can be designed by adding the two filters in parallel to a common junction. A T-Junction is a three port lossless reciprocal device which connects two $\mathrm{TE}_{10}$ mode waveguide channel filters to the common input port [19]. The phase length between input of each filter and common port determines the isolation of each filter in opposite filter pass band. The length of the common junction is needed to be optimised in order to provide isolation between outputs of the side ports. The common junction is an extra section of ceramic placed in between two ceramic waveguide filters. The input coupling from common junction to each individual filter branch is achieved by metal plated through holes in the broad wall of the ceramic waveguide. The offset of holes from sidewalls of the waveguide is optimised to realize right amount of coupling. A coaxial probe is placed in the common junction to achieve external coupling as shown in Figure 6.16. 


\subsubsection{External coupling}

Coaxial probes of $50 \Omega$ impedance are used for all external couplings. A coaxial probe placed at the centre of the broad wall excites the fundamental $\mathrm{TE}_{10}$ mode in the rectangular waveguide filter. Probe position from the shorted back end of the filter and its depth inside the waveguide determine the amount of coupling achieved and centre frequency of the coupling probe [79]. Radius of coaxial line determines the power handling capability of the probe. The characteristic impedance and its maximum peak power handling capability can be related as

$$
\begin{gathered}
Z_{0}=\frac{1}{2 \pi} \sqrt{\frac{\mu_{0} \mu_{r}}{\epsilon}} \ln \left(\frac{D_{c}}{d_{c}}\right) \\
P_{\text {peakmax }}=\frac{\left[E_{\text {peak }} d_{c} \ln \left(\frac{D_{c}}{d_{c}}\right)\right]^{2}}{2 Z_{0}}
\end{gathered}
$$

Where $Z_{0}$ is the characteristic impedance, $E_{\text {peak }}$ is peak E-field, $D_{c}$ is outer radius and $d_{c}$ is inner radius of the probe. Coaxial probes with different depth inside the waveguide are well matched at the outputs of narrowband TX and RX filter channels. But for the common junction simple probe at the centre of the waveguide broad wall does not provide significant matching for widely spaced TX and RX channel filters with a separation of $400 \mathrm{MHz}$. Thus, a broadband coax-to-waveguide transition is needed to excite both channels simultaneously with a good match in each pass band. Several design techniques are available in the literature $[80,81]$ to achieve broad band coupling of coax to waveguide transition. In an air-filled waveguide, adding a metal disk of bigger diameter than primary probe diameter enhances the bandwidth achieved [81]. Another way to enhance the coupling bandwidth of the probe is to add another dielectric material of low relative permittivity around the centre conductor which concentrates the E-field around the probe [80]. But these techniques to achieve wider coupling bandwidth are not suitable for solid ceramic waveguide. In ceramic case bandwidth can be enhanced by changing the probe position from centre of the waveguide broad wall towards side wall [80]. Off centre probes have superior coupling bandwidths as compared to centred probes but this off centre probes can excite higher order modes in the waveguide. 


\subsection{EM simulation results}

Figure 6.16 shows the physical layout of a monolithic integrated ceramic waveguide diplexer and its response is shown in Figure 6.17. Doubly terminated waveguide filters are designed first and then they are connected in parallel through a common junction. The common junction is the extra ceramic piece which sits between TX and RX filter. The common coaxial probe is placed in this junction to achieve the input couplings to both filters. Each filter section is coupled through metal plated through holes (shunt inductors) placed between common junction and each individual filter. Each filter section and the common junction are optimised individually to reduce the EM simulation time and afterwards an optimisation of full diplexer was carried out in HFSS $^{\mathrm{TM}}$ EM simulator. As there is a guard band of more than $300 \mathrm{MHz}$ between passband edge frequencies of the RX and TX filter, so only a slight tuning of first cavity and input coupling was required. The length of the input probe inside the waveguide used at common junction is optimised to provide wideband optimal return loss in both TX and RX passband. The bandwidth of the probe is increased by moving its position from the centre of the waveguide towards the side wall of the waveguide. To obtain a better return loss and to mitigate the effects of physical tolerances and material discrepancies, tuning screws can be added to the design. Metal tuning screws can be placed at the centre of each resonator to get the post production tuning capability.

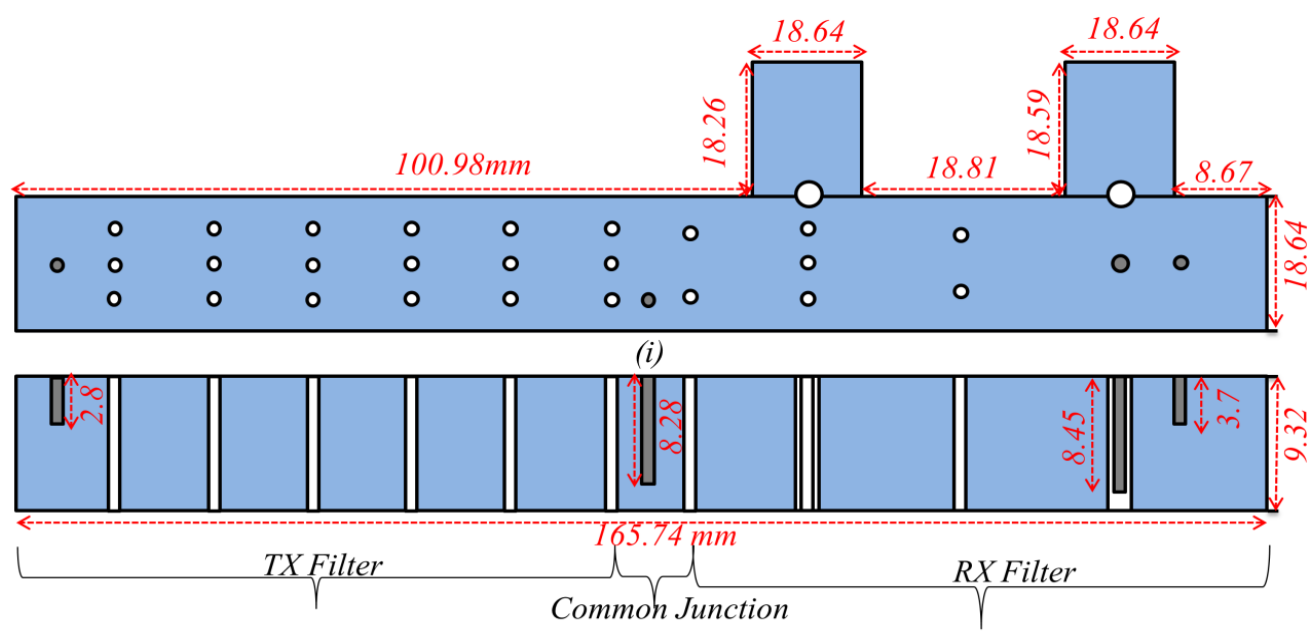

(ii)

Figure 6.16: Ceramic rectangular waveguide diplexer (i) Top view (ii) Side view 


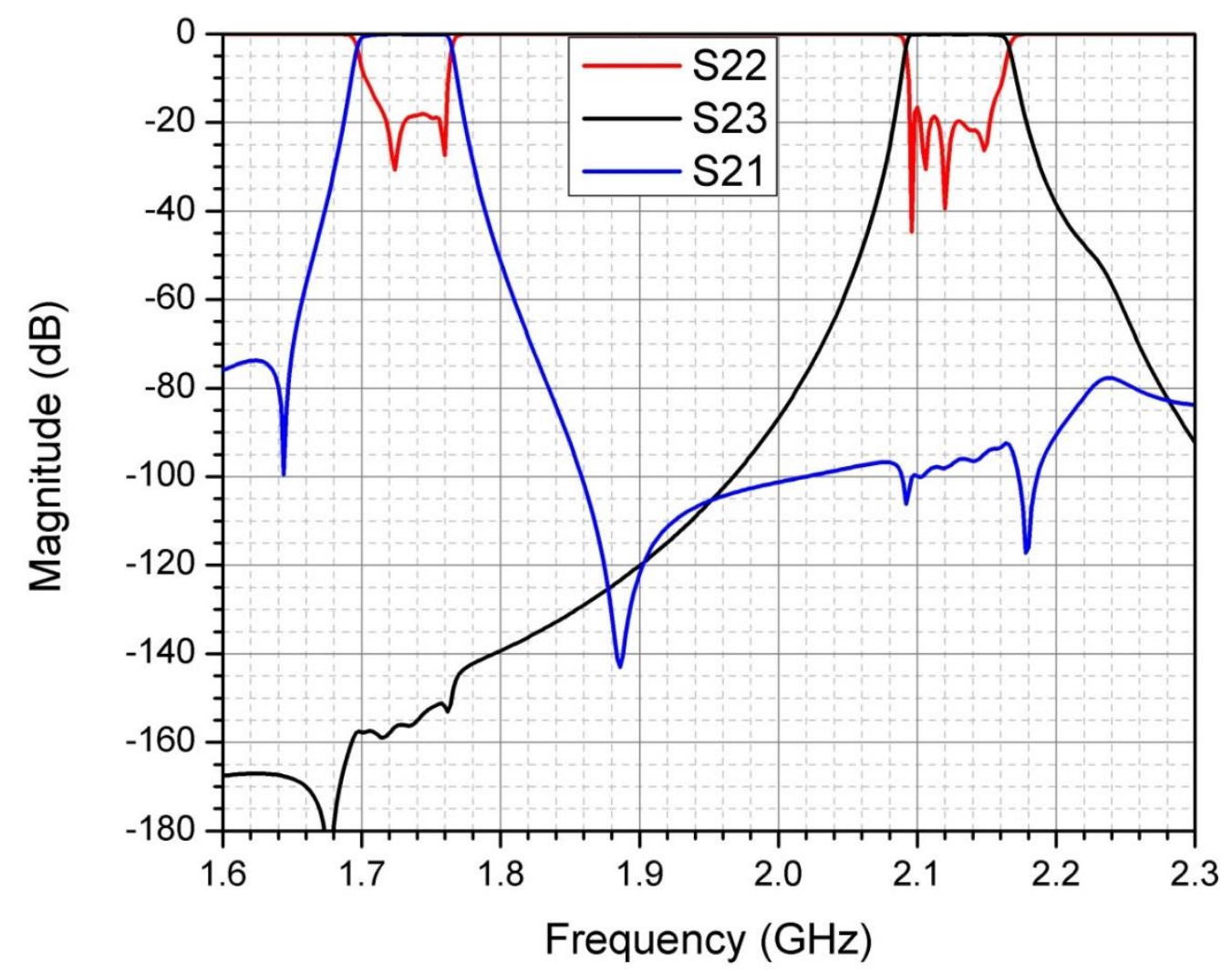

Figure 6.17: Simulated ceramic waveguide diplexer response (HFSS ${ }^{\mathrm{TM}}$ )

\subsection{Summary}

A miniaturized monolithic integrated ceramic waveguide diplexer is designed suitable for DCS base station transceiver. Ceramic waveguide diplexer consists of silver coated ceramic block $\left(\varepsilon_{r}=43\right)$ with different through and blind holes to realize various coupling structures. A six section Chebyshev filter at $2.13 \mathrm{GHz}$ centre frequency with $60 \mathrm{MHz}$ bandwidth for transmit band and a six section generalized Chebyshev filter at $1.73 \mathrm{GHz}$ for receive band is combined through a common junction. External couplings to and from ceramic waveguide diplexer are achieved using coaxial probes. The bandwidth of the common junction probe is improved by offsetting the probe from the centre of the waveguide broad dimension. To achieve post production tuning capability and to get well matched diplexer response, metal screws can be added to each resonator section of the diplexer. 


\section{CERAMIC WAVEGUIDE LOWPASS}

\section{FILTER}

\subsection{Introduction}

Bandpass filters used in cellular communication systems limit the system noise and reduce the out of band interference but in most cases higher order modes are excited easily in these filters. A wide spurious free stopband is generally required in order to remove any out of band interference or intermodulation products. A waveguide lowpass corrugated waveguide filter offers a high power wide spurious free stopband attenuation by suppressing the higher order modes but it is bulky at low frequencies. Therefore a miniaturized integrated ceramic rectangular waveguide lowpass filter is designed to be used with the diplexer in cellular base station front end to provide very wide spurious free bandwidth. This much miniaturized low loss device can be integrated with the ceramic diplexer designed in Chapter 6 to provide a very wide spurious free rejection.

The corrugated waveguide filters are the equivalent of common ladder type series inductor and shunt capacitor lumped element filter. The corrugations in the waveguide are small as compared to the quarter wavelength of operating frequency. The low and high impedance levels used in LC ladder circuit can be realized by varying the height of the waveguide where series inductance $(\mathrm{L})$ or high impedance corresponds to bigger height of the waveguide and shunt capacitance $(\mathrm{C})$ can be realized by lowered height waveguide section. The pass band of the corrugated waveguide low pass filter extends from a cut-off frequency $\left(f_{c}\right)$ of the waveguide to the cutoff frequency of the filter $\left(f_{1}\right)$. Although having a low pass filter arrangement but due to its waveguide nature all the 
frequency components are rejected below cutoff frequency of the waveguide.

The waveguide modes with variations in the height of the waveguide usually cannot propagate through corrugated waveguide filter due to the presence of the corrugations in waveguide height. Therefore higher order modes with $\mathrm{TE}_{\mathrm{mn}}$ field pattern can only come through the stop band of the corrugated rectangular waveguide filter operating in its fundamental $\mathrm{TE}_{10}$ mode [82]. If corrugated low pass waveguide filter is excited by a probe at the center of the waveguide, any even order mode cannot be excited, as all even order TE modes have minimum E-field at the center of the waveguide broad wall. Therefore $\mathrm{TE}_{30}$ is the first spurious mode for a rectangular corrugated waveguide filter [20]. This mode can be cancelled by varying the length or height of the high impedance waveguide sections [83]. The air-filled waveguide low pass filter has very low loss as compared to other low pass filter technologies because of higher Q and it can handle more power levels but it has three main disadvantages as compared to other low pass filter technologies, for example coaxial low pass filter [20]

I. It is more bulky as compared to coaxial low pass filter

II. It is more expensive as compared to coaxial low pass filter

III. Its spurious performance is worse than the coaxial low pass filter. Various higher order modes can be easily excited to waveguide corrugated low pass filter

Another way to suppress higher order modes in a waveguide low pass filter is to introduce longitudinal slots in the low impedance level section of the corrugated waveguide. This arrangement of low pass waveguide filter is called "Waffle Iron Filter" [84]. Waffle iron filter can have spurious free stop band up to three times the cutoff frequency of the filter at the expense of the complexity of the filter. If very wide stop band is not required then corrugated waveguide filter is the right choice as it is easier to manufacture as compared to waffle iron filter. A fast circuit based synthesis of low pass corrugated waveguide filter is presented in [85]. The low pass waveguide filter can be designed based on insertion loss or image parameter filter design method explained in the later section. Figure 7.1 shows a typical arrangement of corrugated waveguide low pass filter.

The volume of the overall low pass waveguide corrugated filter can be reduced by a factor of $\frac{1}{\varepsilon_{r} \sqrt{\varepsilon_{r}}}$ by replacing air filled empty waveguide structure by an integrated piece 
of corrugated ceramic waveguide having $\varepsilon_{r}=43$. The pass-band insertion loss of the corrugated ceramic rectangular waveguide low pass filter is increased by a factor of $\frac{1}{\sqrt{\varepsilon_{r}}}$ as compared to empty air filled waveguide low pass filter. In this Chapter a corrugated ceramic waveguide low pass filter is designed based on image parameter method of filter design. The designed filter is a much miniaturized integrated device suitable to be used at the cellular base stations along with band pass filter to provide very wide stop band.

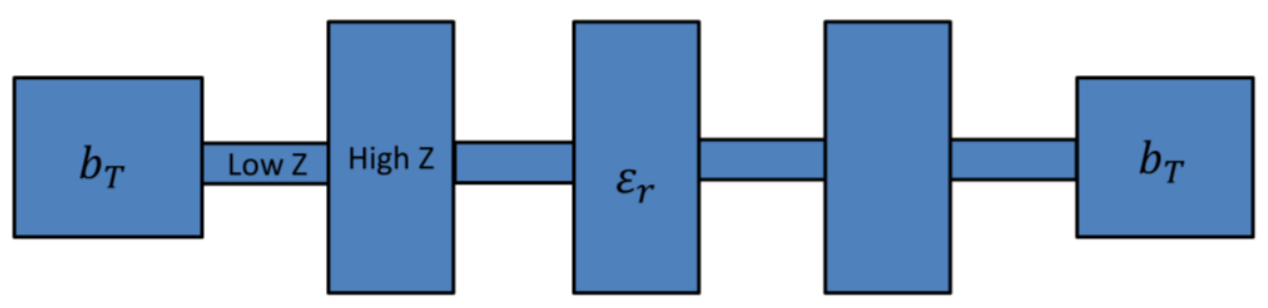

(i)

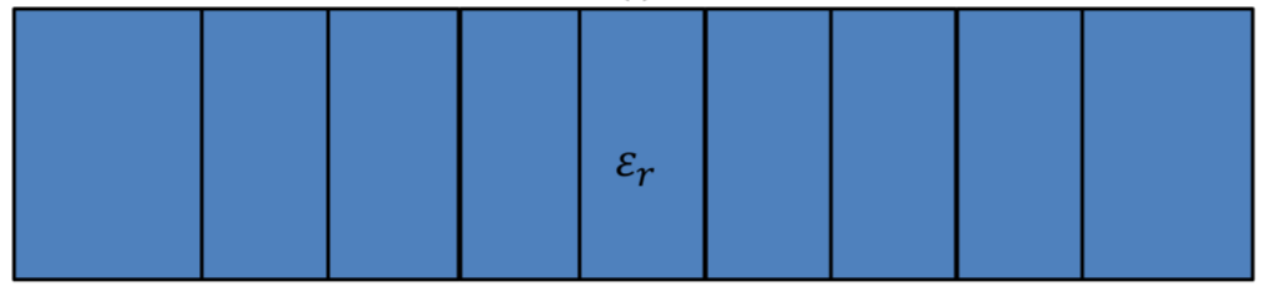

(ii)

Figure 7.1: Corrugated waveguide low pass filter layout (i) Side View (ii) Top view

\subsection{Image parameter filter design method}

Image parameter method of filter design is relatively simple and it takes account of stopband and passband characteristics for a cascade of two port networks. The filter analysis starts with the calculation of image impedances which for a two port network as shown in Figure 7.2 are defined as

$\mathrm{Z}_{\mathrm{i} 1}=$ input impedance at port 1 when port 2 is terminated with $\mathrm{Z}_{\mathrm{i} 2}$

$\mathrm{Z}_{\mathrm{i} 2}=$ input impedance at port 2 when port 1 is terminated with $\mathrm{Z}_{\mathrm{i} 1}$

With both ports matched when terminated in their image impedances. Solving for $Z_{i 1}$ and $\mathrm{Z}_{\mathrm{i} 2}$ gives [7] 


$$
\begin{aligned}
& Z_{i 1}=\sqrt{\frac{A_{A B C D} B_{A B C D}}{C_{A B C D} D_{A B C D}}} \\
& Z_{i 2}=\sqrt{\frac{B_{A B C D} D_{A B C D}}{A_{A B C D} C_{A B C D}}}
\end{aligned}
$$

And the propagation factor of the network can be derived as [7]

$$
e^{-\gamma}=\sqrt{A_{A B C D} D_{A B C D}}-\sqrt{B_{A B C D} C_{A B C D}}
$$

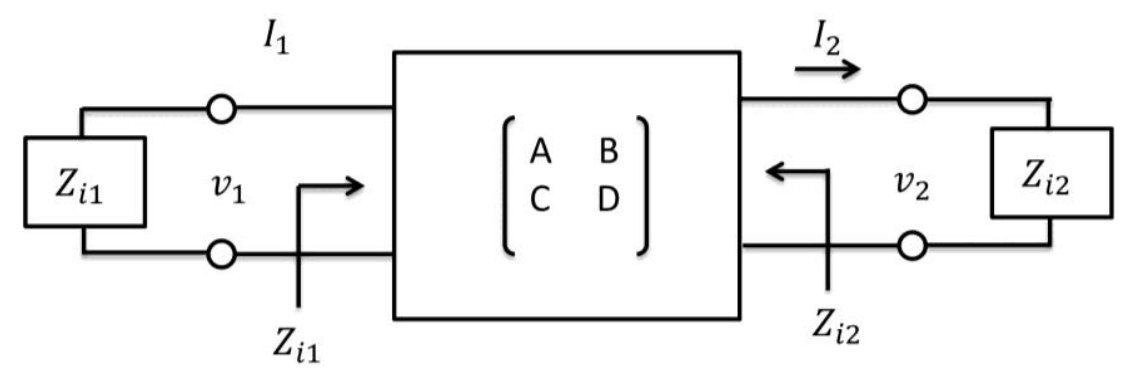

Figure 7.2: Two port network terminated in its image impedance

Two port networks could be in $\mathrm{T}$ or $\pi$ form. The low pass filter designed using image parameter method consists of constant- $\mathrm{K}_{\mathrm{LPF}}$ filter sections or $\mathrm{m}$-derived filter sections as shown in Figure 7.3. The constant- $\mathrm{K}_{\mathrm{LPF}}$ filter section is the basic section of composite filter. The series inductance and shunt capacitance both tend to block the high frequency components and the network behaves as low pass filter. Constant- $\mathrm{K}_{\mathrm{LPF}}$ section provides moderate out of band rejection and roll of rate near cut-off frequency of the filter. While parallel resonant circuit in series branch of $\mathrm{m}_{\mathrm{LPF}}$-derived section introduces a transmission zero near the cut-off frequency of the filter which provides the sharp pass band to stop band transition. The position of attenuation pole is controlled by the parameter ' $0<\mathrm{m}_{\mathrm{LPF}}<1$ '. As ' $\mathrm{m}_{\mathrm{LPF}}$ ' approaches to ' 1 ' the $\mathrm{m}_{\mathrm{LPF}}$-derived section becomes constant- $\mathrm{K}_{\mathrm{LPF}}$ section with attenuation pole at infinity. Smaller values of $\mathrm{m}_{\mathrm{LPF}}$ produce transmission zero near cut-off of the filter with sharp transition. Sharp cut-off could be achieved with single $\mathrm{m}_{\mathrm{LPF}}$-derived section but the stop band rejection of $\mathrm{m}_{\mathrm{LPF}}$-derived section is poor past the attenuation pole frequency. Therefore, practical filter could 
consist of several cascaded constant- $\mathrm{K}_{\mathrm{LPF}}$ sections with $\mathrm{m}_{\mathrm{LPF}}$-derived sections [7].

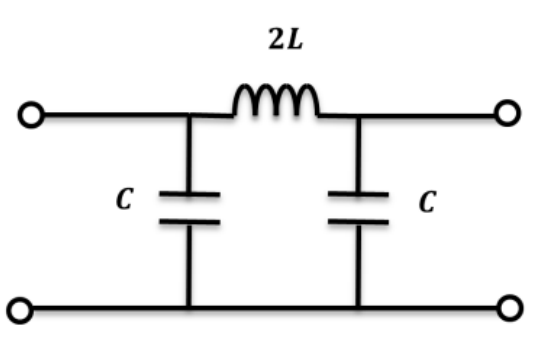

(a)

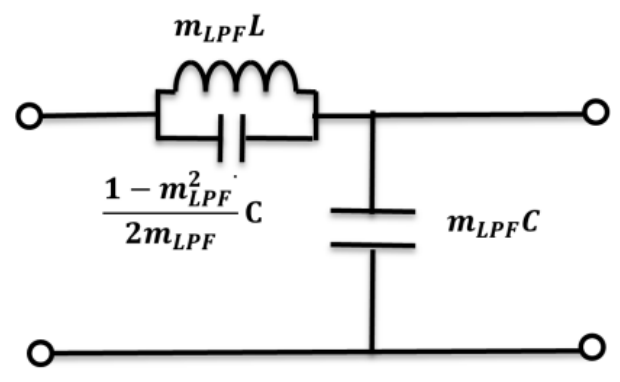

(b)

Figure 7.3: (a) Constant K-section (b) m-derived half section of low pass filter

For the constant k-section, only three parameters are needed to be calculated i.e. series inductance $\mathrm{L}$, shunt capacitance $\mathrm{C}$ and image impedance $\mathrm{R}_{0}$ at zero frequency. The $\mathrm{L}$ and $\mathrm{C}$ values depend on cut-off frequency of the filter and can be calculated as

$$
L=\frac{2 z_{O}}{\omega_{c}}
$$

And

$$
C=\frac{2}{z_{0} \omega_{c}}
$$

The image impedance of the filter sections is a function of frequency therefore to obtain a good match in the pass band $m_{L P F}$-derived sections are included in the filter. The $m_{L P F}$ -derived sections with $\mathrm{m}_{\mathrm{LPF}}=0.6$ are used at filter ends to obtain the flat pass band impedance of the filter [7]. For sharp cut-off m-derived sections $m_{\text {LPF }}$ can be calculated as

$$
m_{L P F}=\sqrt{1-\left(\frac{f_{1}}{f_{\text {in }}}\right)^{2}}
$$

Where $f_{1}$ is the cut-off frequency of the filter and $f_{\text {in }}$ is the attenuation pole frequency. A circuit diagram of a low pass filter with five cascaded constant- $K_{L P F}$ sections with two $m_{L P F}$-derived half sections at the ends is shown below in Figure 7.4 and its simulated response is shown in Figure 7.5. An attenuation pole is introduced in the response due to placement of two $m_{L P F}$-derived half sections. The value of $m_{L P F}$ determines the position of attenuation pole placed in the stopband of the filter. Table 7-1 summarizes the low pass filter component values. 


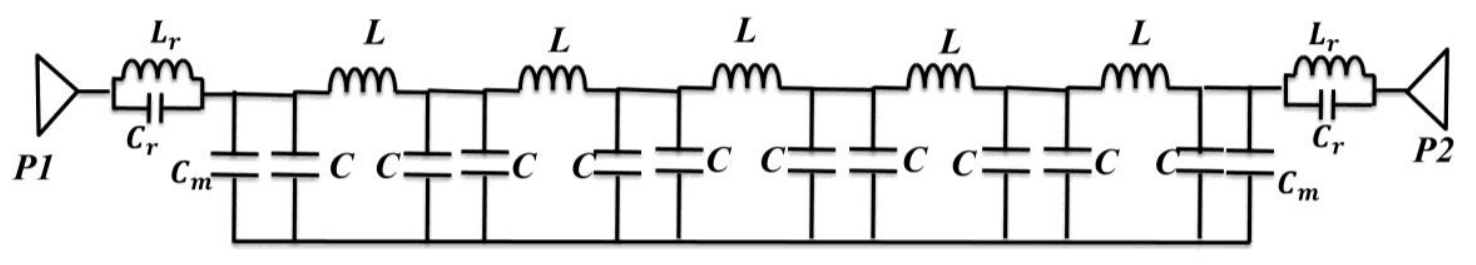

Figure 7.4: Low pass filter circuit diagram

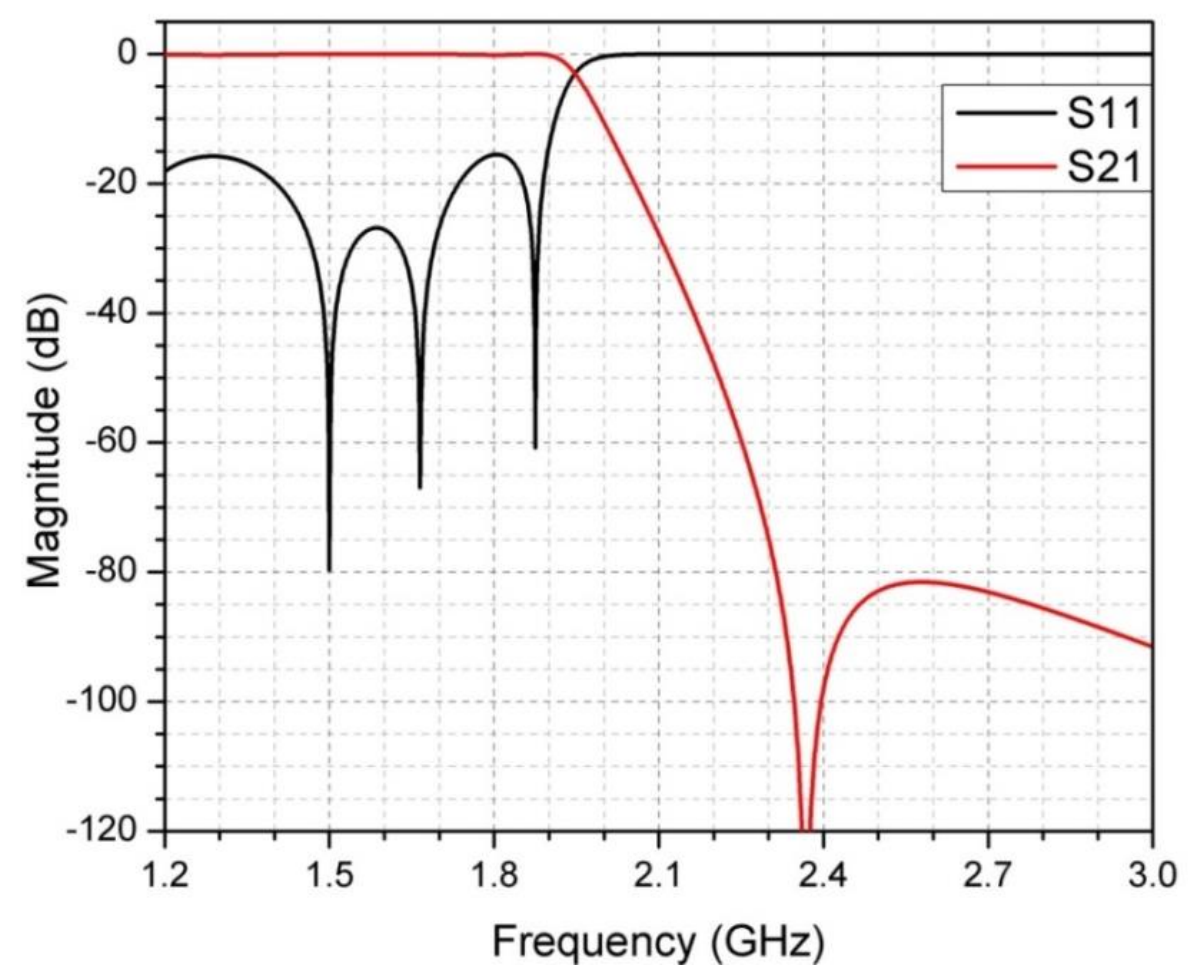

Figure 7.5: Simulated low pass filter response

Table 7-1 : Low pass filter circuit element values

\begin{tabular}{|c|c|c|}
\hline Component & Value & Unit \\
\hline $\mathrm{L}$ & 5.635 & $\mathrm{nH}$ \\
\hline $\mathrm{C}$ & 2.31 & $\mathrm{pF}$ \\
\hline $\mathrm{L}_{\mathrm{r}}$ & 1.2 & $\mathrm{nH}$ \\
\hline $\mathrm{C}_{\mathrm{r}}$ & 3.76 & $\mathrm{pF}$ \\
\hline $\mathrm{C}_{\mathrm{m}}$ & 0.81 & $\mathrm{pF}$ \\
\hline
\end{tabular}




\subsection{Corrugated waveguide LPF design procedure}

A low pass corrugated ceramic waveguide filter shown in Figure 7.1 can be designed based on image parameter filter design method developed by Cohn [20]. Low pass ceramic corrugated waveguide filter offers wide stop band and low loss in the pass band operating in its fundamental propagation mode. The equivalent circuit of a single half section of low pass corrugated waveguide filter is shown in Figure 7.6. Where, $\mathrm{Y}_{01}$ and $\mathrm{Y}_{02}$ are normalized characteristic admittances and $\mathrm{Y}_{\mathrm{I}}$ is the normalized image admittance. $B_{C 1}$ is the series suceptance, $B_{c 2}$ is shunt suceptance, $b_{o c}$ and $b_{s c}$ are open and short circuit susceptances, respectively.

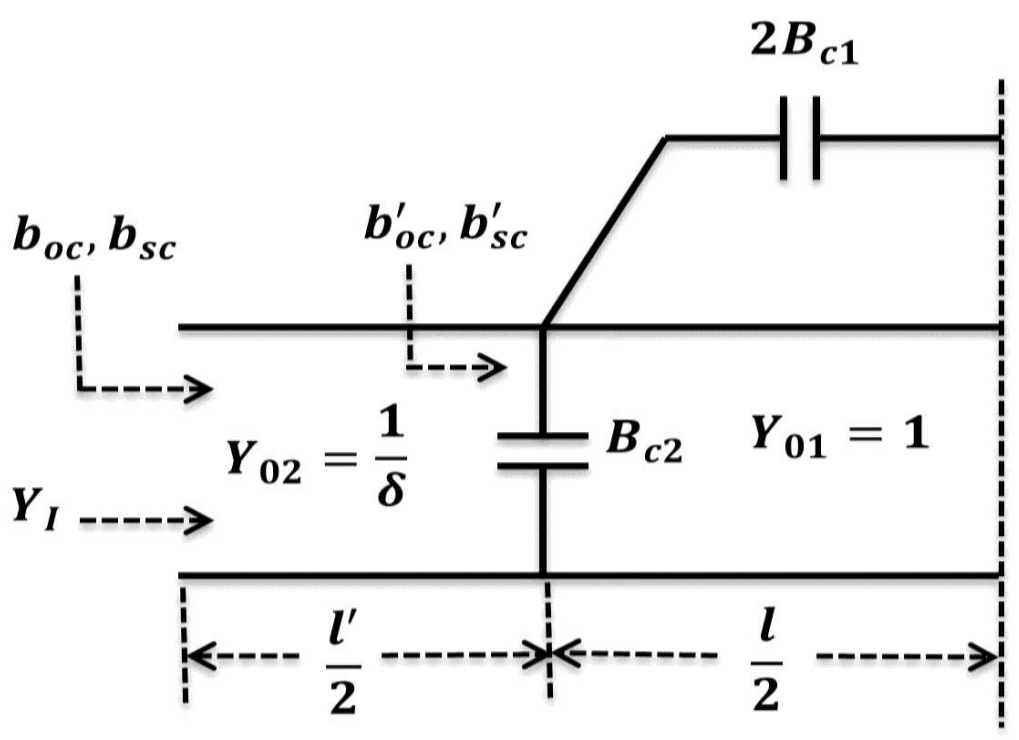

Figure 7.6: Corrugated waveguide half section

The characteristics of low pass corrugated waveguide filter depend only on wavelength of $\mathrm{TE}_{\mathrm{n} 0}$ mode due to uniform corrugations along the width of the waveguide. Admittances of the waveguide sections are normalized with respect to characteristic admittance of higher impedance waveguide section. Therefore, terminating waveguide sections have normalized characteristic impedance of $b_{\mathrm{WG}} / b_{\mathrm{T}}$ where $b_{\mathrm{WG}}$ and $b_{\mathrm{T}}$ are defined in Figure 7.7. The open and short circuit susceptances of half section of corrugated waveguide filter can be calculated as [20] 


$$
b_{0 c}=\frac{1}{\tau} \tan \left[\frac{\pi l_{W G}^{\prime}}{\lambda_{g}}+\tan ^{-1}\left(\tau b_{o c}^{\prime}\right)\right]
$$

And

$$
b_{s c}=\frac{1}{\tau} \tan \left[\frac{\pi l_{W G}^{\prime}}{\lambda_{g}}+\tan ^{-1}\left(\tau b_{s c}^{\prime}\right)\right]
$$

Where

$$
b_{0 c}^{\prime}=\tan \left(\frac{\pi l_{W G}}{\lambda_{g}}\right)+B_{c 2}
$$

And

$$
b_{s c}^{\prime}=-\cot \left(\frac{\pi l_{W G}}{\lambda_{g}}\right)+B_{c 2}+2 B_{c 1}
$$

And

$$
\tau=\frac{b_{W G}^{\prime}}{b_{W G}}
$$

When $\tau \leq 0.15$, the shunt susceptance $\mathrm{B}_{\mathrm{c} 2}$ can be evaluated by the expression

$$
\begin{aligned}
B_{c 2}=\frac{2 b_{W G}}{\lambda_{g}}\{ & \ln \frac{1}{\tau}-0.338 \\
& \left.+\sum_{h=1}^{\infty}\left[\frac{\tanh \frac{h \pi l_{W G} F}{b_{W G}}}{F}-1\right]\right\}-0.09 \frac{b_{W G}}{\lambda_{g}}
\end{aligned}
$$

And the series suceptance $\mathrm{B}_{\mathrm{c} 1}$ can be expressed as

$$
B_{c 1}=\frac{2 b_{W G}}{\lambda_{g}} \sum_{h=1}^{\infty}\left[\frac{\operatorname{csch} \frac{2 h \pi l_{W G} F}{b_{W G}}}{F}\right]
$$

Where

$$
F=\sqrt{1-\left(\frac{b_{W G}}{h \lambda_{g}}\right)^{2}}
$$

The normalized image admittance $Y_{I}=\sqrt{Y_{o c} Y_{S C}}$ is 


$$
Y_{I}=j \frac{\cot \frac{\theta^{\prime}}{2}}{\delta} \sqrt{\frac{\left(b_{0 c}^{\prime}+\frac{\tan \frac{\theta^{\prime}}{2}}{\tau}\right)\left(b_{s c}^{\prime}+\frac{\tan \frac{\theta^{\prime}}{2}}{\tau}\right)}{\left(b_{s c}^{\prime}-\frac{\cot \frac{\theta^{\prime}}{2}}{\tau}\right)\left(b_{o c}^{\prime}-\frac{\cot \frac{\theta^{\prime}}{2}}{\tau}\right)}}
$$

And the image propagation constant for a full section is

$$
\begin{gathered}
\gamma=\alpha+j \beta=2 \tanh ^{-1} \sqrt{\frac{Y_{0 c}}{Y_{s c}}} \\
\gamma=2 \tanh ^{-1} \sqrt{\frac{\left(b_{0 c}^{\prime}+\frac{\tan \frac{\theta^{\prime}}{2}}{\tau}\right)\left(b_{s c}^{\prime}-\frac{\cot \frac{\theta^{\prime}}{2}}{\delta}\right)}{\left(b_{0 c}^{\prime}-\frac{\cot \frac{\theta^{\prime}}{2}}{\tau}\right)\left(b_{s c}^{\prime}-\frac{\tan \frac{\theta^{\prime}}{2}}{\tau}\right)}}
\end{gathered}
$$

Where $\theta^{\prime}=\frac{2 \pi l_{W G}^{\prime}}{\lambda_{g}}$ is the electrical length of low impedance section of waveguide with physical length $l_{W G}^{\prime}$. If the waveguide corrugations are small as compared to guide wavelength then an approximation for attenuation per section of a low pass corrugated waveguide filter can be written as [20]

$$
\alpha=17.372 \cosh ^{-1} \frac{\lambda_{\mathrm{g} 1}}{\lambda_{\mathrm{g}}}(\mathrm{dB} / \mathrm{section})
$$

Where $\lambda_{g}$ is the guide wavelength at the specified stop band frequency. The image cutoff frequency $f_{1}$ where image admittance is zero can be obtained from the condition [20]

$$
b_{s c}^{\prime}+\frac{\tan \frac{\theta^{\prime}}{2}}{\tau}=0
$$

And $\mathrm{f}_{\text {in }}$ can be obtained from the condition

$$
b_{S C}^{\prime}=b_{o c}^{\prime}
$$

The upper edge frequency of the first stop band can be evaluated from

$$
b_{s c}^{\prime}-\frac{\cot \frac{\theta^{\prime}}{2}}{\tau}=0
$$


The values of $b_{S c}^{\prime}$ and $b_{o c}^{\prime}$ can be used from the tables of equivalent circuit of waveguide E-plane T-junction by Marcuvitz [78]. Once $f_{c}, f_{1}$ and $f_{\text {in }}$ are determined one can use the graphs from [20] to obtain $b_{W G}, b_{W G}^{\prime}, l_{W G}$ and $l^{\prime}$ values. The image admittance in the pass band of the filter normalized to high impedance section height can be approximated as

$$
Y_{I}=\frac{b_{W G}}{b_{0 W G}} \sqrt{1-\left(\frac{\lambda_{g}}{\lambda}\right)^{2}}
$$

Where $\lambda_{g 1}$ is the guide wavelength at $f_{1}$. The terminating waveguide height can be adjusted to give a perfect match at a mid-band frequency of $f_{0}$ where guide wavelength becomes $\lambda_{g 0}$ as [20]

$$
b_{T}=\frac{b_{0 W G}}{\sqrt{1-\left(\frac{\lambda_{g 1}}{\lambda_{g 0}}\right)^{2}}}
$$

If $b_{T}=1.428 b_{0}$, a fairly good match can be obtained over entire pass band bandwidth. For further enhancement of impedance matching, quarter wave impedance transformers can be used. To compensate the susceptance (B) effect between terminating waveguide $\left(\mathrm{b}_{\mathrm{T}}\right)$ and the reduced height section connecting terminating guide with length $l_{W G}^{\prime} / 2$ and height $b_{W G}^{\prime}$, this line must be reduced by an amount of $\Delta l_{W G}^{\prime}$ approximated as [20]

$$
\Delta l_{W G}^{\prime}=\frac{b_{W G}^{\prime}}{2 \pi}\left[\frac{\lambda_{g}}{b_{T}} \frac{B}{Y_{O}}\right]
$$

Where $\mathrm{Y}_{0}$ represents the characteristics admittance of the terminating line.

\subsection{Simulated Results for GSM1800}

\subsubsection{Corrugated waveguide lowpass filter}

A $5^{\text {th }}$ order corrugated ceramic waveguide low pass filter is designed with a cut-off frequency of $1.9 \mathrm{GHz}$ and pass band bandwidth of $600 \mathrm{MHz}$. A $2 \mathrm{GHz}$ spurious free stop band with a minimum attenuation of $60 \mathrm{~dB}$ is achieved extending from $2.25 \mathrm{GHz}$ to $4.15 \mathrm{GHz}$. Figure 7.7 shows the layout of the ceramic waveguide corrugated low pass 
filter and the simulation variables are given in Table 7-2.

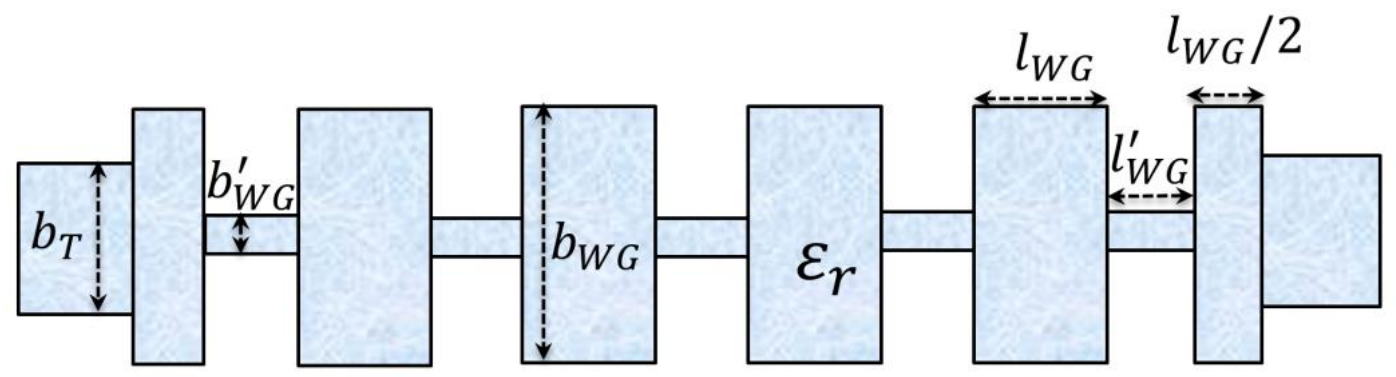

(Side View)

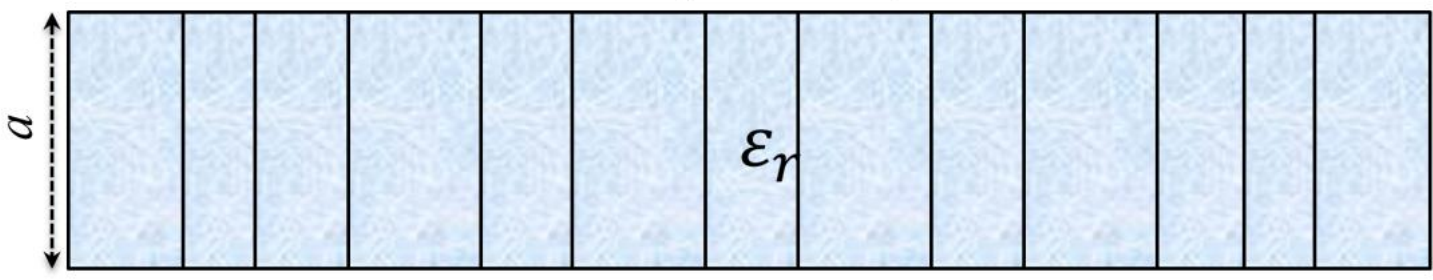

(Top View)

Figure 7.7: Corrugated waveguide low pass filter

Table 7-2 : Low pass corrugated ceramic waveguide filter dimensions

\begin{tabular}{|c|c|c|}
\hline Variable & Value $(\mathrm{mm})$ & Description \\
\hline$l_{W G}$ & 2.6 & High impedance section length \\
\hline$l_{W G}^{\prime}$ & 1.8 & Low impedance section length \\
\hline$b_{W G}$ & 10 & High impedance section height \\
\hline$b_{W G}^{\prime}$ & 1 & Low impedance section height \\
\hline$b_{T}$ & 2.2 & Terminating waveguide height \\
\hline $\mathrm{a}$ & 18 & Width of the waveguide \\
\hline
\end{tabular}

Figure 7.8 shows the ceramic corrugated waveguide low pass filter response and detailed passband insertion loss is given in

Figure 7.9. These graphs show that a low loss ceramic corrugated waveguide lowpass filter exhibits wide spurious free stopband. 


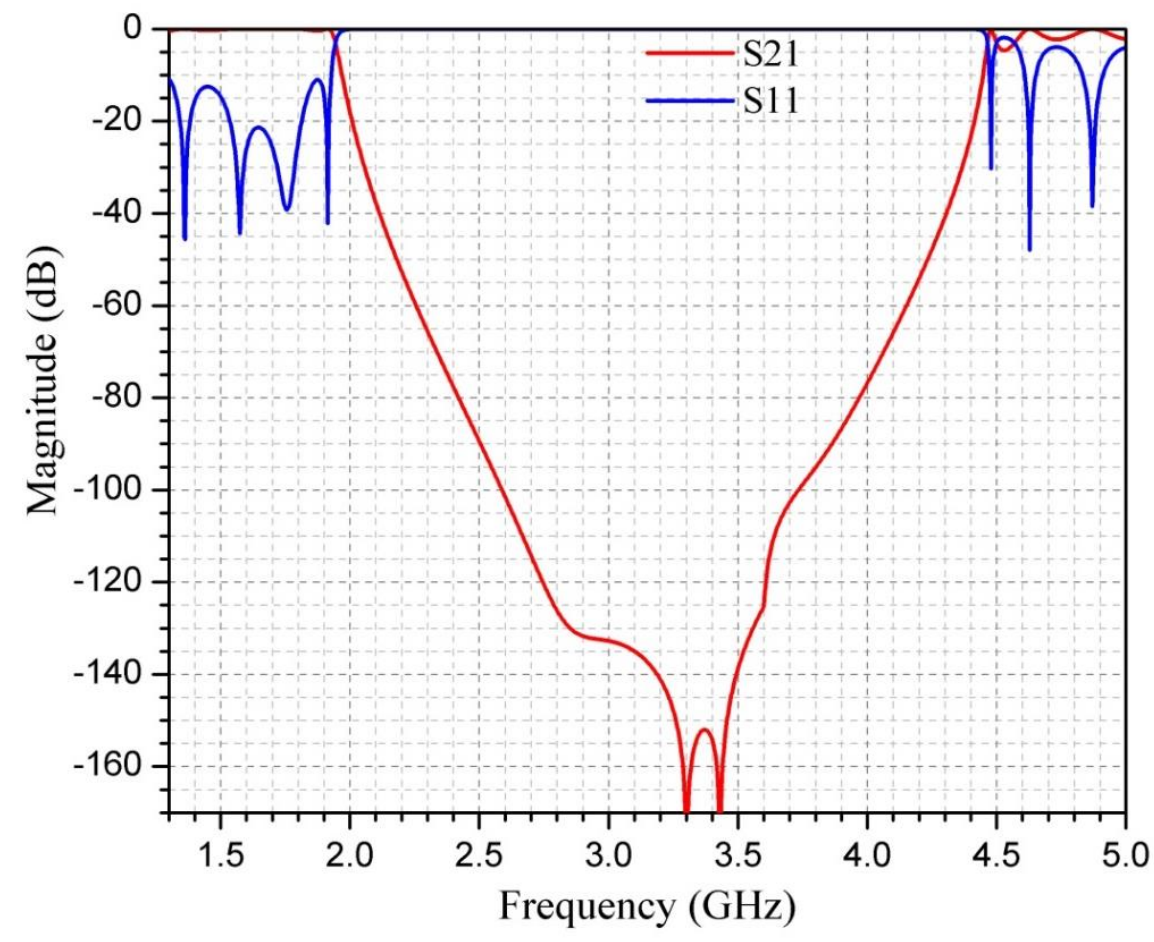

Figure 7.8: Simulated corrugated ceramic waveguide low pass filter response (HFSS ${ }^{\mathrm{TM}}$ )

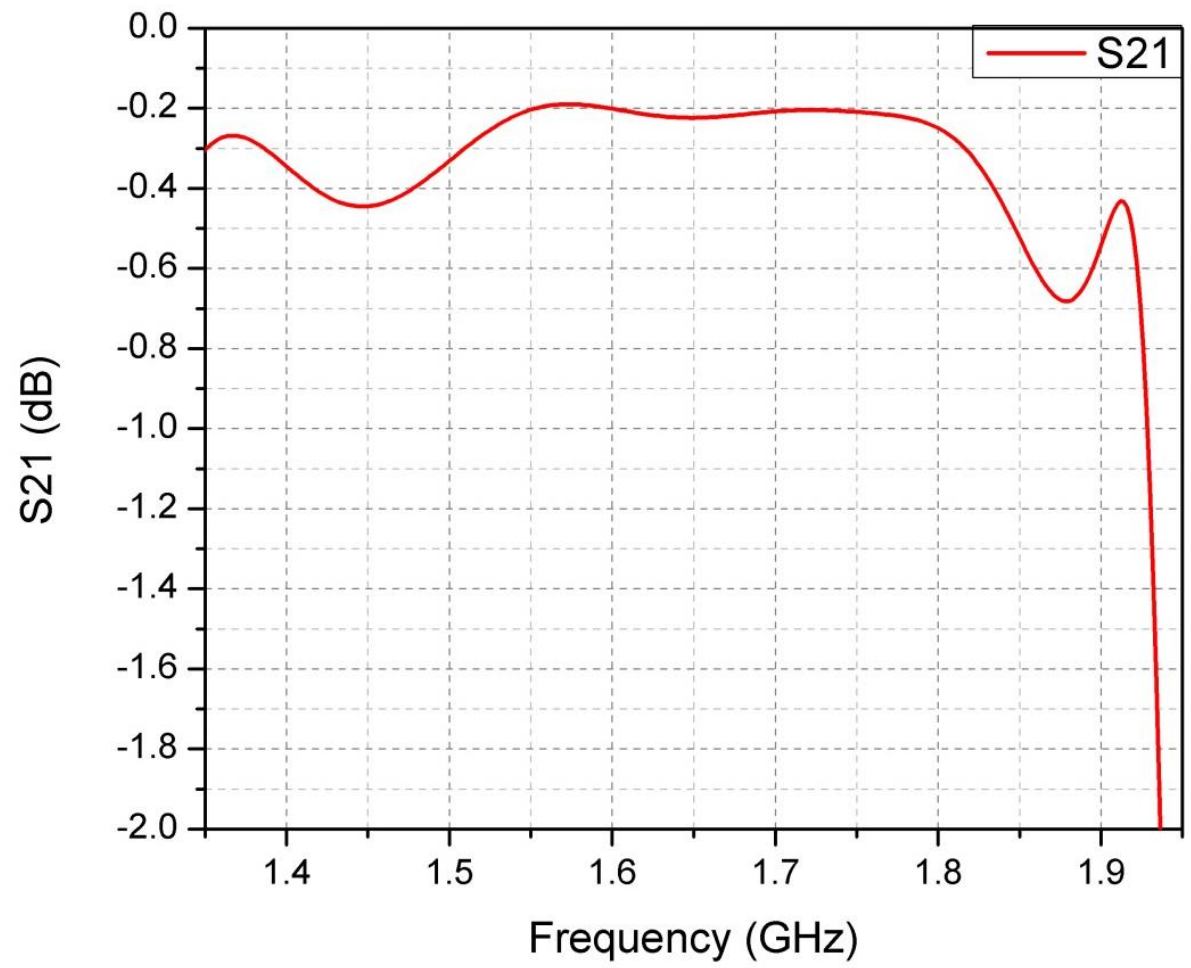

Figure 7.9: Simulated passband IL of ceramic corrugated waveguide filter $\left(\mathrm{HFSS}^{\mathrm{TM}}\right.$ ) 


\subsubsection{Improved spur corrugated waveguide LPF}

If we assume a single section of the corrugated waveguide filter as $m_{\mathrm{LPF}}$-derived low pass filter section, as shown in Figure 7.6. There exist two types of capacitances, a shunt capacitance $B_{C 2}$, due to electric field concentration between upper and lower edges of the reduced height section of the waveguide, and a series capacitance across the high impedance line due to the electric field concentration between neighbouring reduced height sections. Thus, a parallel combination of capacitance and inductance formed by high impedance line in the series arm of the filter produces an attenuation pole in the stop band of the filter at $f_{\text {in }}$. The position of this pole can be changed by varying the capacitance or inductance of the parallel resonant circuit in the series arm. This can be achieved by varying the height or length of the high impedance section which changes the inductance or capacitance of the resonant circuit, respectively. Therefore, the position of $f_{\text {in }}$ can be controlled by varying either height or length of the high impedance section of the low pass ceramic corrugated waveguide filter.

To achieve wider spur free stop band, the attenuation pole $f_{\text {in }}$ can be placed between $f_{2}$ and $f_{3}$ where the next harmonic pass band occurs. This eliminates the pass band between $f_{2}$ and $f_{3}$ and a wider spurious free stop band is achieved. Although by varying the dimensions of higher impedance section, the cut-off frequency of the filter also varies, but this change in cut-off frequency is small as compared to change in next higher order mode resonance. Figure 7.10 shows the change in cut-off frequency $\left(f_{1}\right)$, infinite attenuation pole frequency $\left(f_{\text {in }}\right)$ and next higher order pass band lower cut off frequency $\left(f_{2}\right)$ as a function of height $\left(b_{\mathrm{WG}}\right)$ of the higher impedance waveguide section while all other parameters are kept constant. It is evident that when $b_{\mathrm{WG}}$ goes from 11 $\mathrm{mm}$ to $7 \mathrm{~mm}$, change in cut-off frequency $\left(f_{1}\right)$ is only $300 \mathrm{MHz}$ while $f_{1}$ changes up to approximately $2 \mathrm{GHz}$. Therefore, another $2 \mathrm{GHz}$ spur free pass band is added to the low pass corrugated waveguide filter. The change in cut-off frequency of the filter can be readjusted by varying the dimensions of the low impedance waveguide section. 


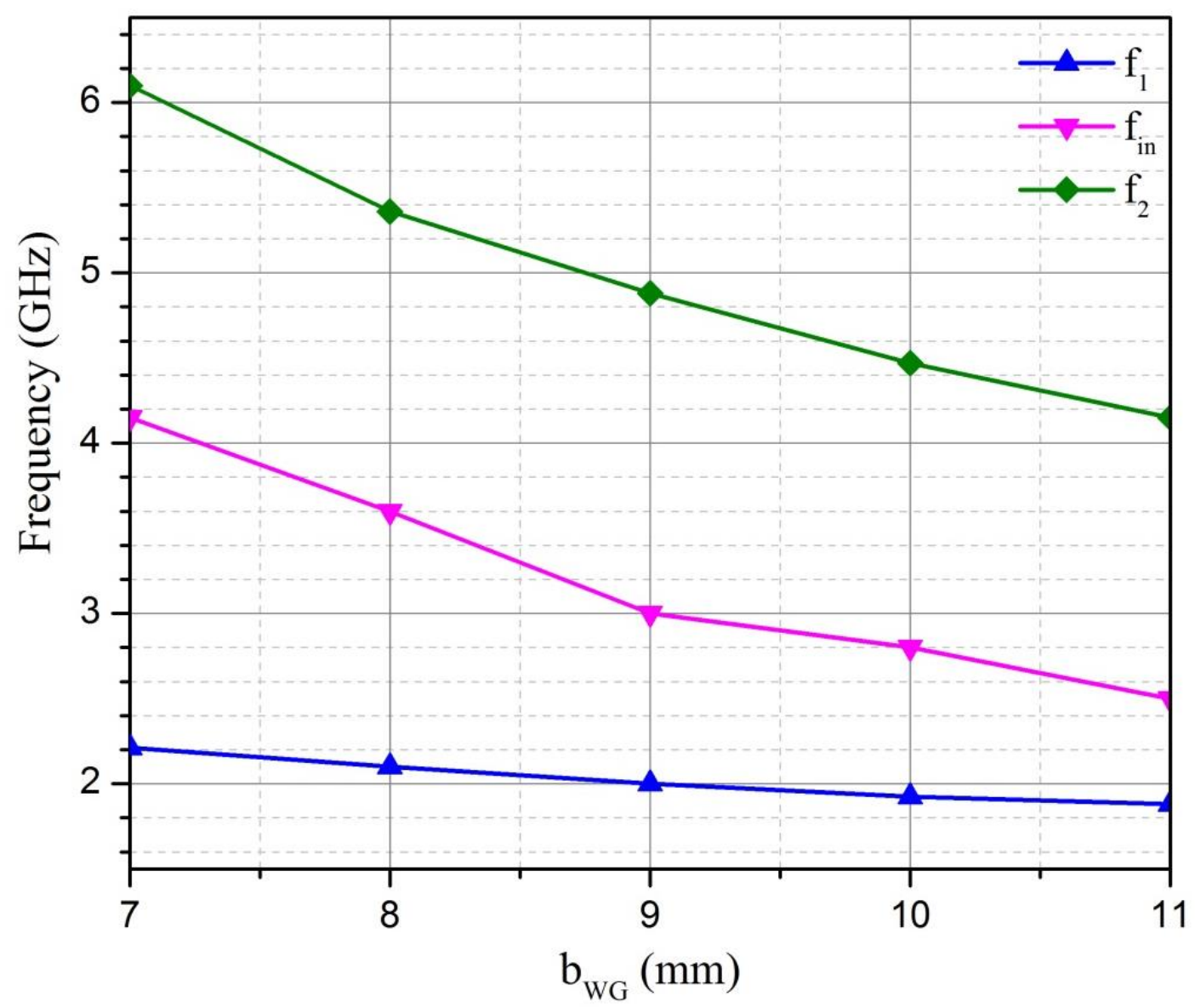

Figure 7.10: Change in cut-off, attenuation pole and spurious resonance frequencies with the height of the high impedance section of corrugated LPF

Figure 7.11 shows the response of low pass corrugated waveguide filter with two different heights of the high impedance waveguide section while all other parameters kept constant. It is clear from the graph that when ' $b_{\mathrm{WG}}$ ' is reduced from $11 \mathrm{~mm}$ to 7 $\mathrm{mm}$, the attenuation pole frequency $f_{\text {in }}$ is moved towards the centre of higher order mode pass band eliminating it completely. Thus, for a minimum $60 \mathrm{~dB}$ stop band attenuation, the stopband bandwidth is almost doubled on the expense of slight difference in the cut-off frequency of the low pass filter which could be re-adjusted easily. Another possibility to achieve wider stopband of low pass corrugated ceramic waveguide filter is to increase the length of the high impedance section of the waveguide. Change in the length in the high impedance section is equivalent to change in series capacitance between neighbouring low impedance waveguide sections. 


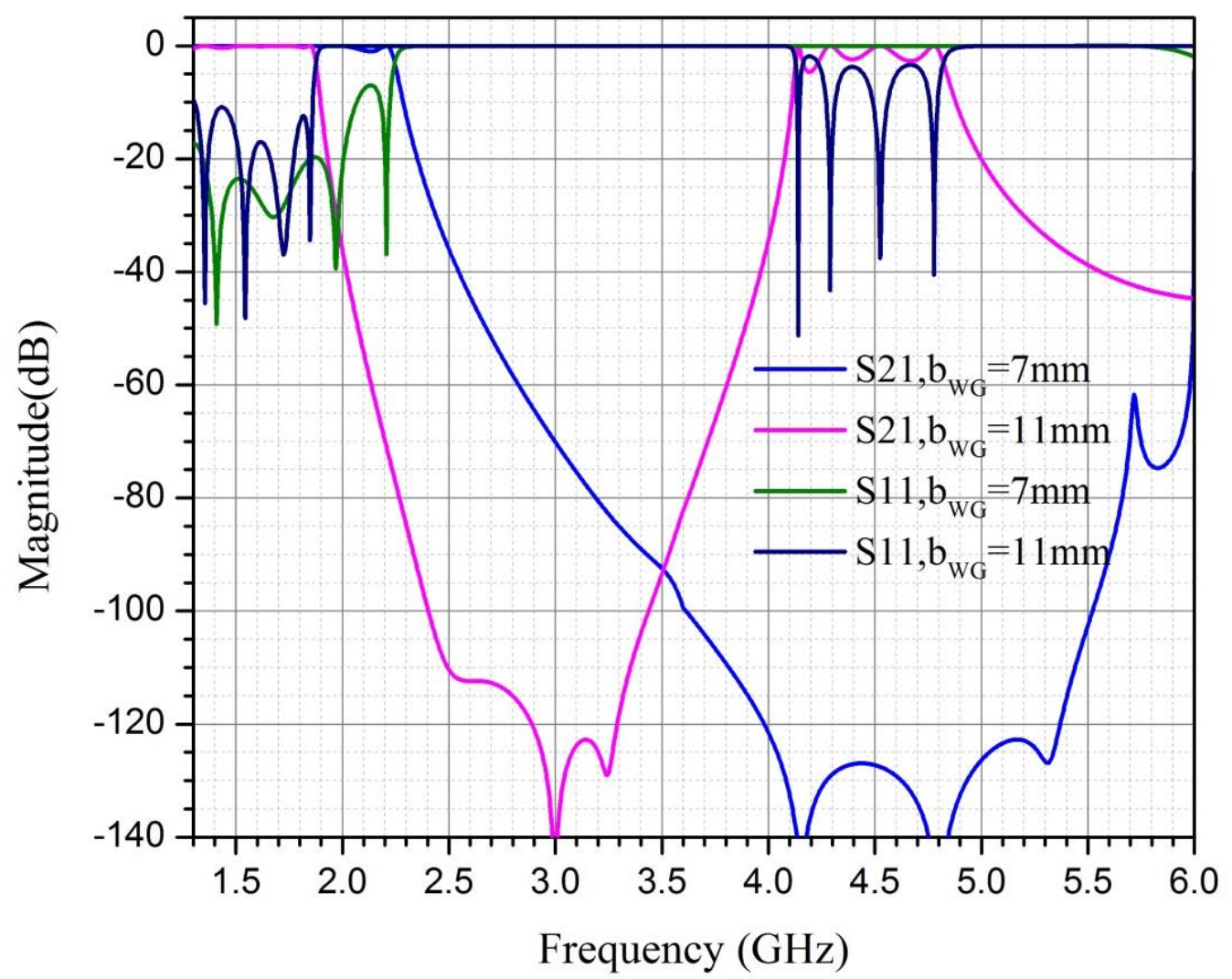

Figure 7.11: Simulated corrugated ceramic waveguide filter response with two different heights of high impedance section $\left(\mathrm{HFSS}^{\mathrm{TM}}\right.$ )

\subsection{Summary}

A ceramic corrugated rectangular waveguide low pass filter is designed. The low pass ceramic waveguide filter can be used along with band pass filter at cellular base stations to meet the wide stop band requirements for transmit and receive path of diplexers. Low pass corrugated ceramic waveguide filter is a much miniaturized device which offers very high stop band rejection. The spurious bandwidth of the low pass corrugated waveguide filter can be further improved by varying the dimension of high impedance section of the waveguide which shifts the attenuation pole towards the higher order spurious resonance. Therefore, higher order spurious mode is completely eliminated providing a wider spur-free stop band bandwidth. Naturally, all the rectangular waveguide structures behave as high pass filter due to zero transmission below cut-off frequency of the waveguide. 


\section{PRACTICAL ISSUES OF CERAMIC}

FILTER DESIGN

\subsection{Introduction}

The integrated ceramic waveguide bandpass filter and diplexer design are described in the previous Chapters. This Chapter addresses some of the practical aspects of the physical design of the integrated ceramic waveguide filter and diplexer. The fabricated filter response may not be exactly the same as simulated results obtained due to mechanical tolerances in the physical dimensions of the fabricated filter or material discrepancies. This Chapter deals with the post production tuning mechanism of ceramic waveguide resonators, power handling capability of the ceramic waveguide filters, spurious performance of the fabricated ceramic waveguide filters and thermal effects on the resonance of ceramic resonators.

\subsection{Ceramic waveguide resonator tuning}

Manufacturing tolerances could lead to a shift in frequency of individual resonators, therefore tuning is always required to get the fabricated filter/diplexer response as close to the simulation as possible. Tuning mechanism allows post fabrication capability to shape the stringent electrical response of the filter. If tuning mechanism is introduced in the filter it can reduce the overhead time to optimize the simulated results to very tight limits. There can be several methods to tune a ceramic waveguide resonator, i.e. tuning by metal screws, ceramic screws or removing metal from the surface of the resonator etc. Tuning of ceramic resonator can be achieved by inserting a ceramic or metallic 
screw at the centre of the broad wall of the resonator. In a ceramic waveguide resonator maximum E-field occurs at the centre of the broad wall of the resonator so a tuning screw at this position will perturb its E-field most and a wide tuning range can be achieved. A hole for the tuning screw will shift the resonant frequency of the resonator and also degrade its Q-factor. The effects of both ceramic and metal tuning screw are described below.

\subsubsection{Tuning with metal screw}

The convenient way to tune the resonant frequency of a ceramic waveguide resonator is to perturb its E-field by placing a metallic tuning screw at the centre of its broad wall. The amount of tuning can be controlled by the depth and the diameter of the tuning screw and that of the non-metalized blind hole to accommodate the tuning screw. To achieve a wider tuning range in $\mathrm{TE}_{10}$ mode of the ceramic waveguide, a tuning screw must be placed at the centre of the broad wall where maximum E-filed is stored. A hole placed at the centre of ceramic resonator will shift its resonance frequency to a higher value and also degrades its Q-factor. A ceramic waveguide resonator with a tuning screw at its centre is shown in the Figure 3.8. The ceramic waveguide resonator shown in Figure 3.8 resonates at a fundamental frequency of $f_{0}$ without any tuning hole in it. When a tuning blind hole is placed in the resonator, its resonant frequency shifts to $f_{0}+\Delta f$. Increasing the depth of metal tuning screw in the blind hole decreases the resonant frequency of the ceramic waveguide resonator. The length (d) of the resonator is optimised with the tuning screw half way in the blind hole to produce a fundamental frequency of $f_{0}$. This allows a two way tuning capability in the fabricated filter. Figure: 8.1 Shows the effect of tuning when a metal tuning screw of different diameter is placed in the ceramic resonator. To get the following results, the diameter of the blind hole is kept $1 \mathrm{~mm}$ bigger than the diameter of the tuning screw and the depth of the blind hole is kept $6 \mathrm{~mm}$ deep in a ceramic $\left(\varepsilon_{r}=43\right)$ waveguide resonator with the dimensions of $18.64 \times 18.64 \times 9.32(\mathrm{~mm})$. If there is no blind hole inserted for the tuning purpose then the ceramic rectangular block with dimensions $18.64 \times 18.64 \times 9.32(\mathrm{~mm})$ resonates at a fundamental frequency of $1732 \mathrm{MHz}$. A wide frequency tuning range of more than 100 $\mathrm{MHz}$ is achieved by using a $6 \mathrm{~mm}$ metal tuning screw. Figure: 8.2 shows that the tuning screw does not have much effect on the Q-factor of the resonator. 


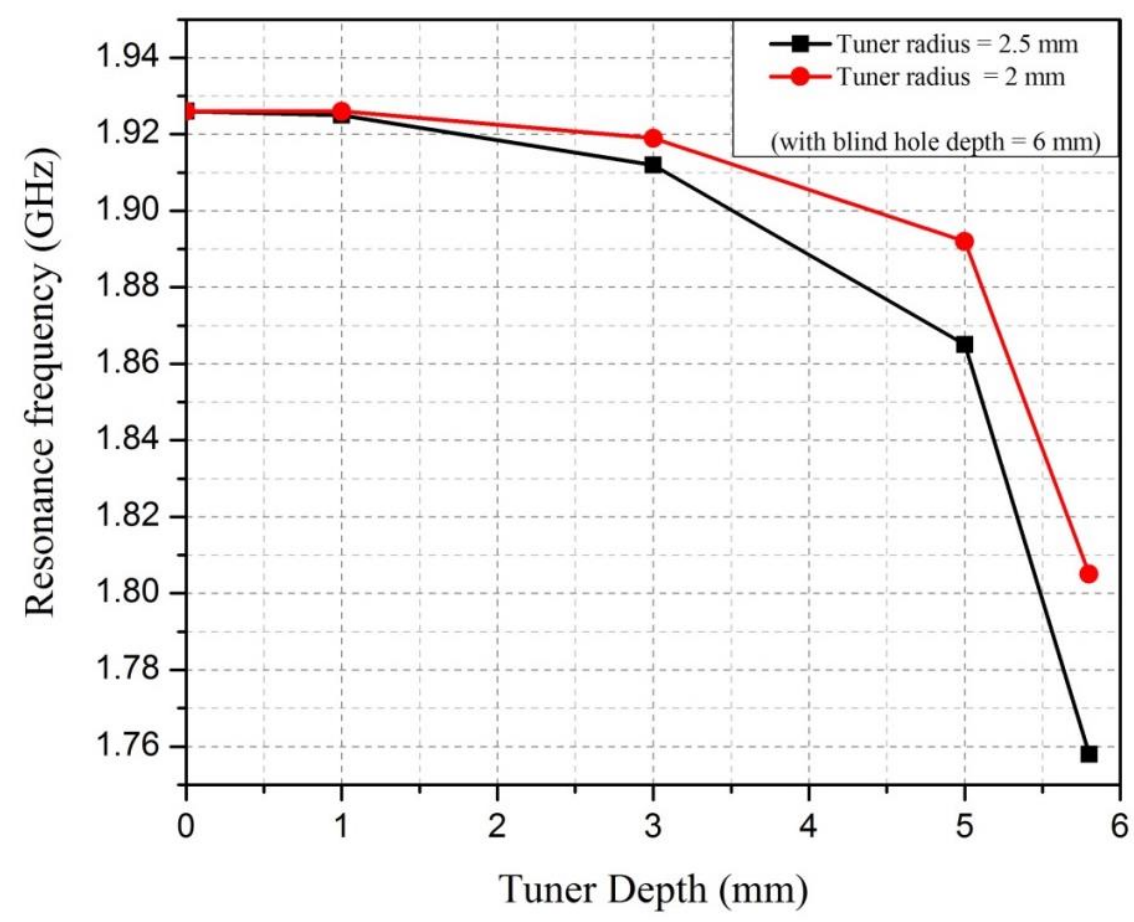

Figure: 8.1: Simulated resonance frequency tuning with metal tuning screw of different diameter

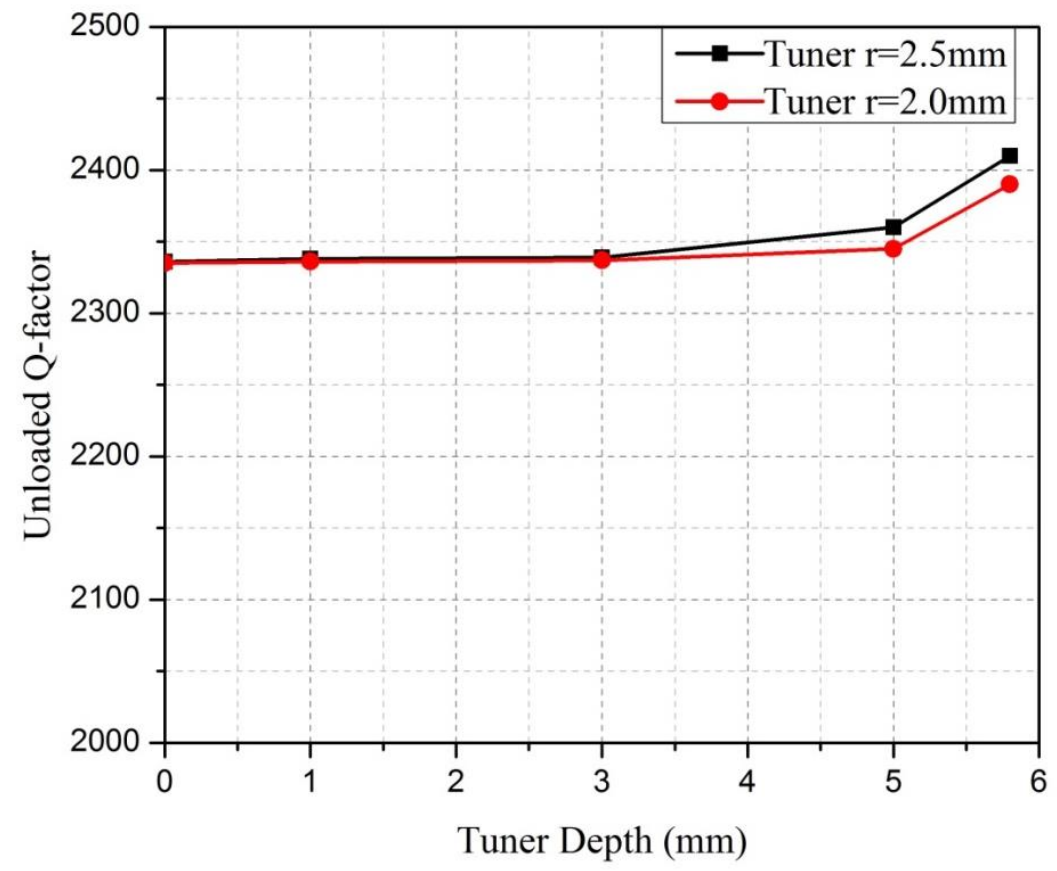

Figure: 8.2: Simulated tuning screw length effect on unloaded Q of the resonator 
Although the insertion of non-metal coated blind hole placed in the ceramic waveguide resonator to accommodate the tuning screw decreases the Q-factor of the ceramic waveguide resonator, yet the depth of the tuning screw inside the non-metalized blind hole does not produce much variation in the overall Q-factor of the resonator. Also the diameter of both tuning screws does not have much effect on the Q-factor but a wider tuning range can be achieved with bigger diameter tuning screw.

\subsubsection{Tuning with ceramic screw}

Another possibility to tune the fundamental frequency of the dielectric waveguide resonator is to replace the metal tuning screw with the ceramic tuner. The depth of the ceramic screw inside the resonator, its diameter, the depth and the diameter of the blind hole determine the amount of tuning achieved. The tuning screw is placed at the centre of the broad wall of the resonator so as to perturb the maximum E-field area of the resonator. The Q-factor of the resonator is decreased when a non-metalized blind hole is placed at the centre of the broad wall of the ceramic resonator to accommodate the tuning screw. Increasing the depth of the ceramic tuner inside the blind hole again increases the Q-factor of the resonator. A ceramic resonator with a ceramic tuning screw in it is shown in the Figure: 8.3.

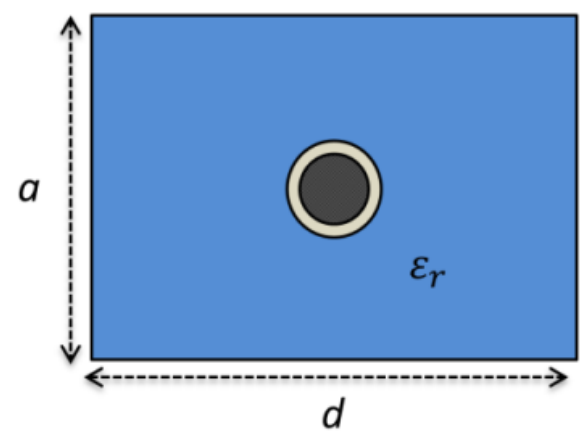

(a)

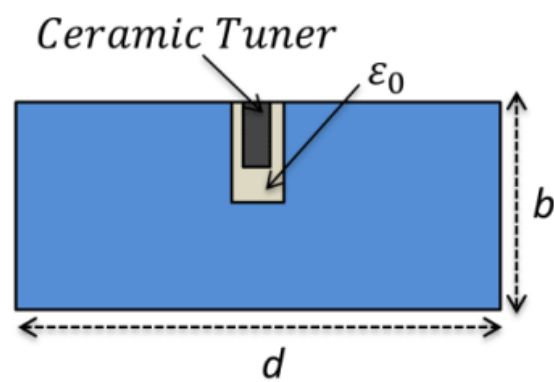

(b)

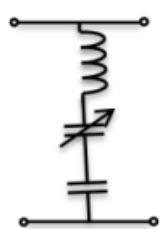

(c)

Figure: 8.3: Ceramic resonator with ceramic tuner (a) Top view (b) Side view (c) Equivalent circuit

The amount of tuning achieved with different diameter ceramic $\left(\varepsilon_{r}=43\right)$ tuning screws is shown in Figure 8.4. The data shown in this graph is obtained by placing a 
non-metalized blind hole of diameter $3 \mathrm{~mm}$ and $6 \mathrm{~mm}$ length placed at the centre of the broad wall of the ceramic $\left(\varepsilon_{r}=43\right)$ waveguide resonator with the dimensions of 18.64 $\mathrm{x} 18.64 \mathrm{x} 9.32(\mathrm{~mm})$. The fundamental resonant frequency of the ceramic waveguide resonator is $1732 \mathrm{MHz}$ if there was no any tuning hole placed in it.

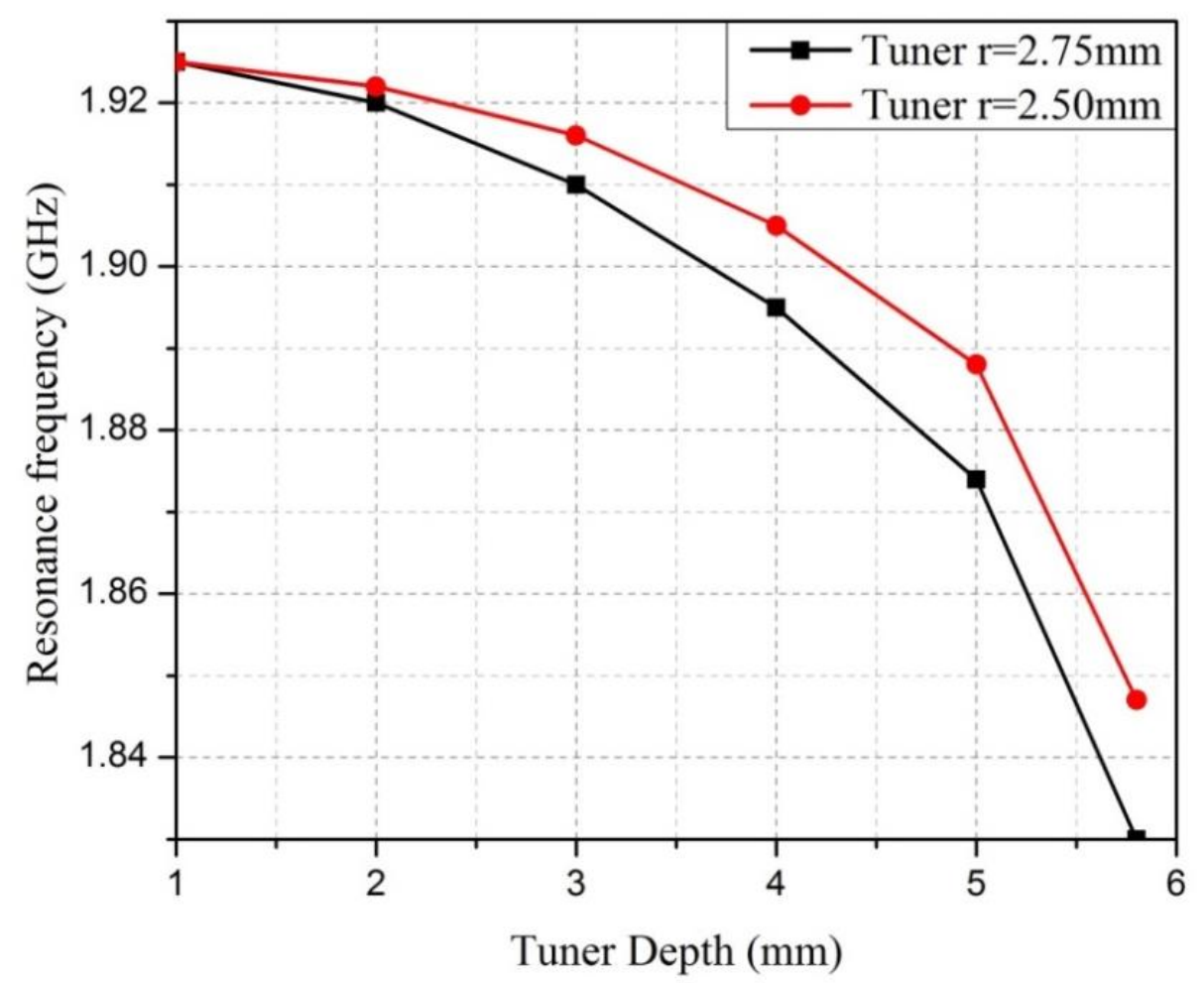

Figure 8.4: Simulated resonance frequency tuning with ceramic tuner

The effect on Q-factor of the resonator by ceramic tuning screw of different diameter is shown in Figure: 8.5. It is observed that the Q-factor of the resonator improves by increasing the length of the ceramic tuning screw inside the blind hole. From the above discussion it is evident that with the same dimensions of tuning hole in the resonator and same radius of the tuner, metal tuning screw achieves wider tuning range as compared to the ceramic screw. Also, there is not much effect on the Q-factor of the resonator by either metal or ceramic screw. Ceramic screw needs smaller gap between the diameter of the blind hole and that of tuning screw to increase the tuning range. On the other hand, metal tuning screws are cheap, easy to manufacture and allow same tuning range with the wider gap between the diameter of the blind hole and that of tuner thus increasing power handling capability of the resonator. 


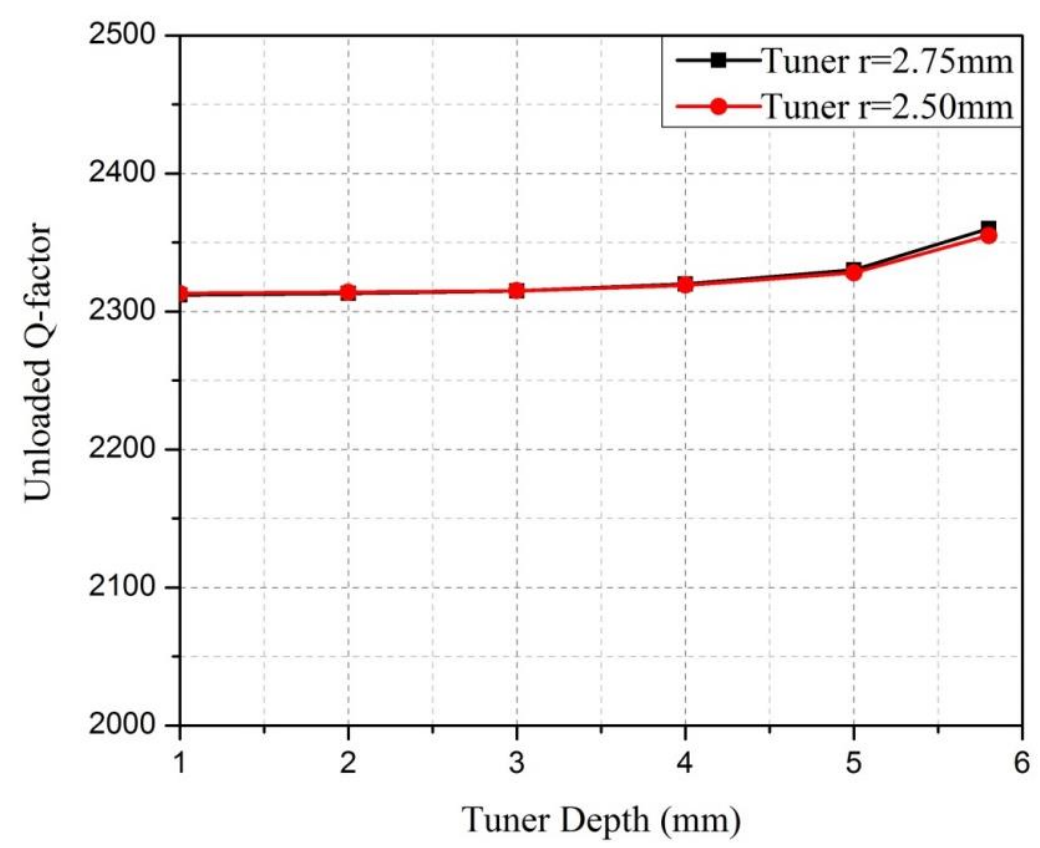

Figure: 8.5: Simulated Ceramic tuner effect on unloaded Q-factor of resonator

\subsection{Manufacturing Process}

To begin the manufacturing process of the ceramic waveguide filter, a mixture of powder of the dielectric material and binders is prepared. This prepared mixture is pressed into block to form the shape. Then, this soft shaped material is cut, machined and prepared to drawing in green state, using machining tools. Then, this prepared part is fired at high temperature to create a hard and dense material. Addition of thermal energy promotes strong bonds between the raw particles of the ceramic. Firing can cause $20 \%-40 \%$ shrinking of the material in green state, which could be accommodated as it is predictable. A secondary machining and lapping is done on fired part to give it the final shape, according to drawing. Finally, metal layer is sprayed on the ceramic part except the input/output probe positions and in the blind holes for tuning screws.

\subsection{Temperature Effects}

There could be three possible effects of the temperature upon the ceramic waveguide filter, i.e. positive or negative expansion of the ceramic material due to the change in temperature, drift in the permittivity of the ceramic material or the thermal expansion of the tuning screw. There is negligible change in the ceramic permittivity or its 
dimensions due to the temperature variation as the temperature coefficient of the ceramic is $-4.5 \mathrm{ppm} /{ }^{0} \mathrm{C}$. There could be a small change in the length of the silver tuning screw due to thermal changes. Thermal expansion of the metal can be expressed as [86]

$$
\Delta L_{l}=\alpha_{l} L_{i l} \Delta T
$$

Where $\alpha_{1}$ is the linear coefficient of thermal expansion, $\Delta \mathrm{T}$ is change in temperature and $\mathrm{L}_{\mathrm{il}}$ is the initial length of silver tuning screw. Thermal coefficient for silver is 19.68 $\mathrm{ppm} /{ }^{0} \mathrm{C}$. When temperature is increased from $0{ }^{0} \mathrm{C}$ to $100{ }^{0} \mathrm{C}$ for a silver tuning screw of initial length of $4 \mathrm{~mm}$, its length expands to $4.0019 \mathrm{~mm}$ resulting only a few $\mathrm{KHz}$ shift in the resonant frequency of the ceramic waveguide resonator. Hence it is observed that temperature variations of $100{ }^{0} \mathrm{C}$ do not have significant effect on the performance of the ceramic waveguide resonator/filter.

\subsection{Power handling}

The simplest way to increase the range and capability of a communication link is to increase its transmitter power. Satellite, radar and radio base stations require high power transmit filters and multiplexers to reject out of band spurious resonances. There has always been a demand of low mass and volume for these radio links thus limiting the power handling capability of the filters and the multiplexers present in the radio link. The power breakdown is caused in a filter when the voltage breakdown occurs in the high electric field region of the filter [20]. Power handling capability is generally worse for narrow band filters as they store more energy in the resonators. The maximum energy in a bandpass filter is stored near its pass band edges making it less robust for power handling. The power breakdown is caused by multipaction effect, ionization breakdown, passive intermodulation (PIM) interferences, detuning or due to thermal instability [87]. Narrower gaps or couplings store more E-field in the gap resulting chance to occur breakdown at the interface of gap [87]. Avoiding inclusion of tuning screws enhance power handling capability of the filter. There should always be a significant margin in input power levels so as to avoid any breakdown in the physical device.

\subsubsection{Power breakdown analysis}

A EM simulation can be carried out to calculate the minimum power required to 
produce threshold breakdown voltage and its position in a microwave filter using $\mathrm{HFSS}^{\mathrm{TM}}$. Once the filter is tuned, input power level is increased until its maximum Efield strength reaches to threshold breakdown point. To avoid computational power and time, different models have been proposed in the literature to calculate power breakdown threshold based on single resonator analysis [88-90]. The power handling capability of a microwave filter can be predicted based on the information of normalized stored energy in each resonator. The maximum stored energy in a resonator can be calculated using following relation

$$
w=\text { Stored Energy }=0.5 \varepsilon_{0} \varepsilon_{\mathrm{r}} \int|\mathrm{E}|^{2} \mathrm{dv}
$$

Here E represents maximum electric field strength in the resonator. For a rectangular waveguide cavity resonator the maximum stored energy can be calculated as [88]

$$
w=\frac{a b d}{8} \varepsilon_{0} \varepsilon_{\mathrm{r}} \mathrm{E}^{2}
$$

Where $a, b$ and $d$ represent the width, height and length of the waveguide resonator. The maximum E-field of the resonator and maximum stored energy can be found using $\mathrm{HFSS}^{\mathrm{TM}}$ field calculator tool. Figure 8.6 shows the E-field graph of the TX filter $\left(f_{0}=2.13 \mathrm{GHz}\right)$ with tuning screws.

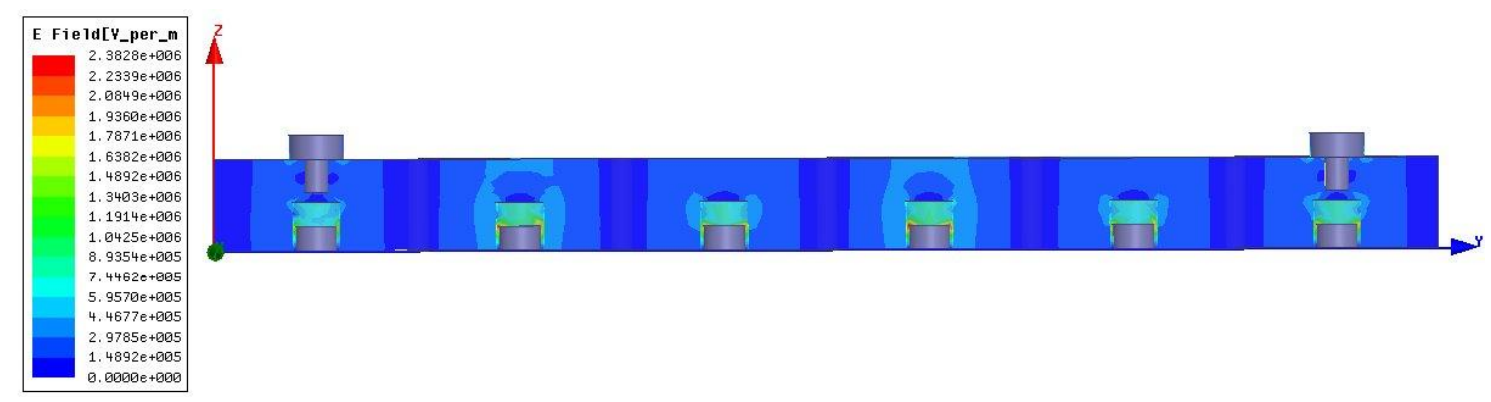

Figure 8.6: Simulated TX filter E-field distribution

It is observed that the maximum E-field strength occurs in the second resonator at the air gap between tuning screw and the resonator. An input power of $2500 \mathrm{~W}$ was required at the input port to produce an air breakdown voltage of $2.3 \mathrm{MV} / \mathrm{m}$ at this point at the centre frequency of $f_{0}=2.13 \mathrm{GHz}$. As the maximum energy is stored near the passband edge, therefore, input power is dropped to $1150 \mathrm{~W}$ to produce the air breakdown voltage of $2.3 \mathrm{MV} / \mathrm{m}$ near the passband edge frequency of $2.158 \mathrm{GHz}$. 
There exists an air gap of $0.3 \mathrm{~mm}$ between ceramic waveguide and tuning screw. If the tuning screws were not present in the design then to produce the same breakdown voltage at the centre frequency of the filter, an input power level of $30 \mathrm{KW}$ was required. As there are small air gaps between tuning screw and the ceramic in a ceramic waveguide filter with tuning screws, therefore its power handling capability reduces significantly. The smaller is the gap between tuning screw and the ceramic resonator the more susceptible is the filter to power breakdown.

\subsection{Spurious performance}

Figure 8.7 and Figure 8.8 show the broadband response of TX and RX filters respectively. It is evident that beside the fundamental pass band there are other spurious modes coupled through the filter. These spurious modes decrease out of band rejection levels. To avoid these unwanted spurious modes a low pass filters could be used along with the band pass filter.

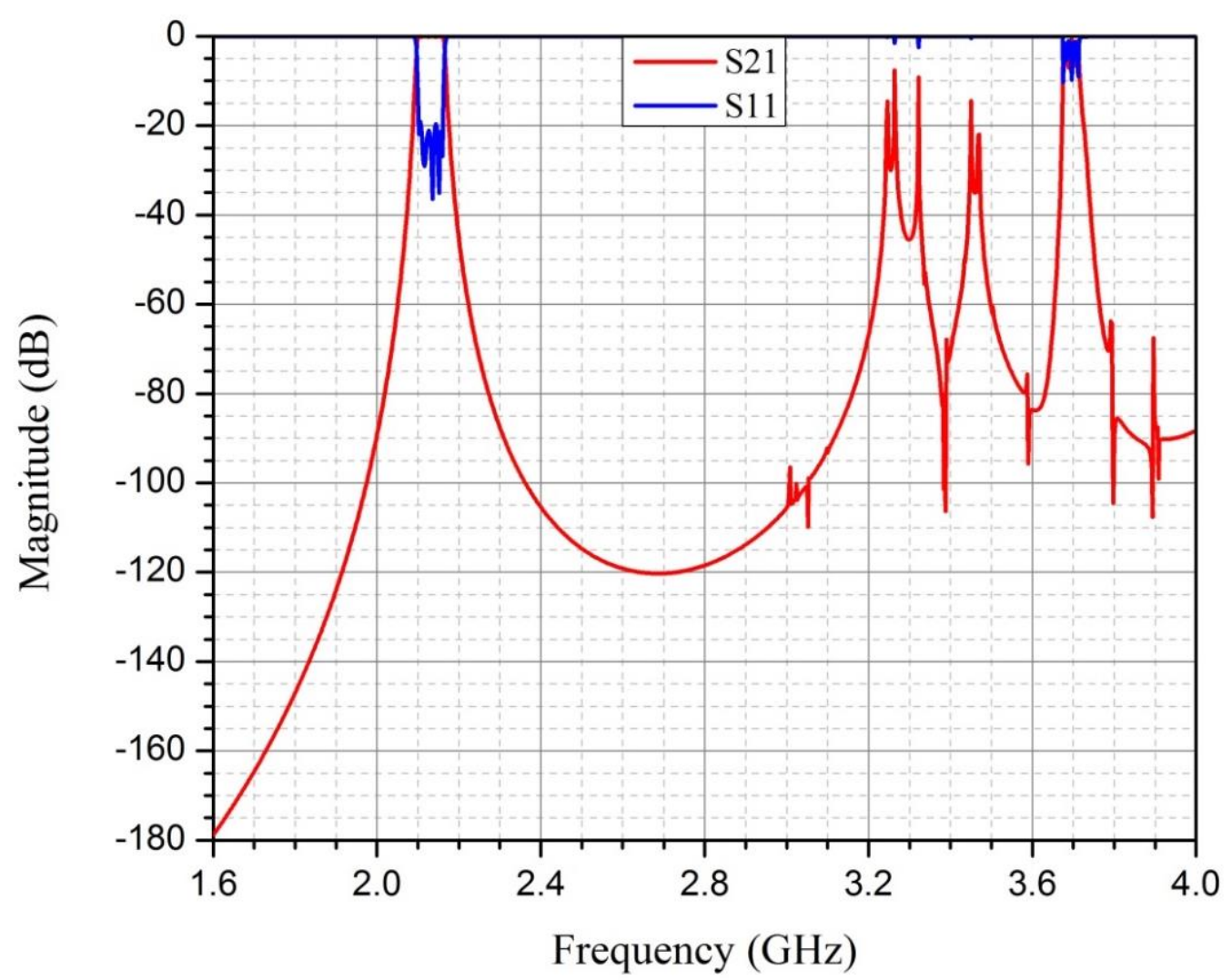

Figure 8.7: Simulated wideband response of TX filter 


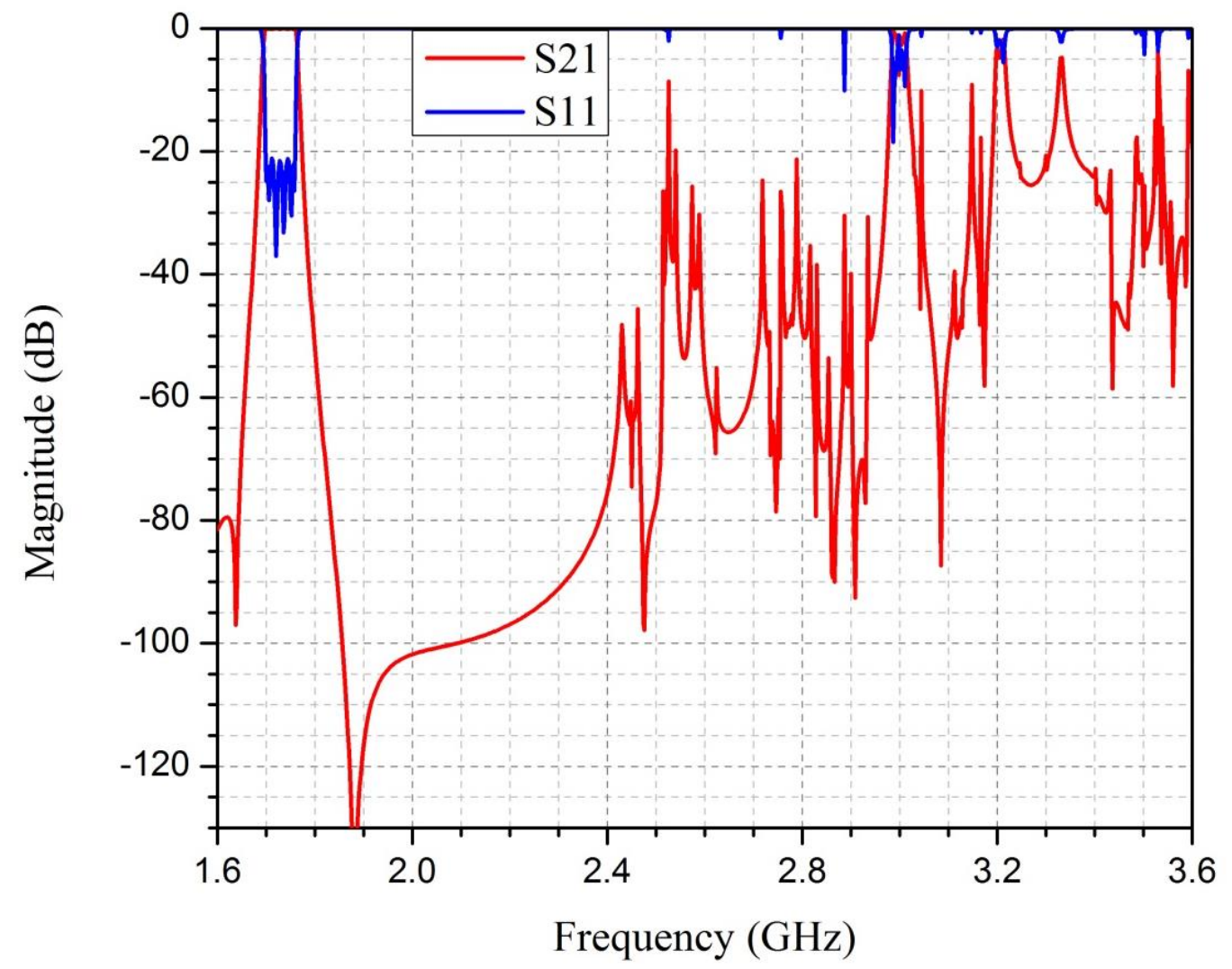

Figure 8.8: Simulated broadband response of RX filter

The spurious performance of the ceramic waveguide filter can be improved by placing a metal coated blind hole at the centre of the resonators. This blind hole shifts the resonant frequency of the resonator below the cut-off, making waveguide resonator as an evanescent mode waveguide resonator. As the depth of the blind hole is increased Qfactor of the resonator and its resonant frequency is decreased. To increase the resonant frequency of the resonator again to $\mathrm{f}_{0}$, the resonator dimensions are decreased. Thus, a silver coated blind hole at the centre of the resonator can improve both the size and spurious performance of the resonator on the expense of lower Q-factor. Figure 8.9 and Figure 8.10 show the effect of blind hole depth on the resonant frequency, first spurious resonance and Q-Factor of the resonator. Figure 8.9 and Figure 8.10 show that by increasing depth of the silver coated blind hole placed at the centre of the broad wall of the ceramic waveguide resonator, resonant frequency and Q-factor of the resonator is decreased. 


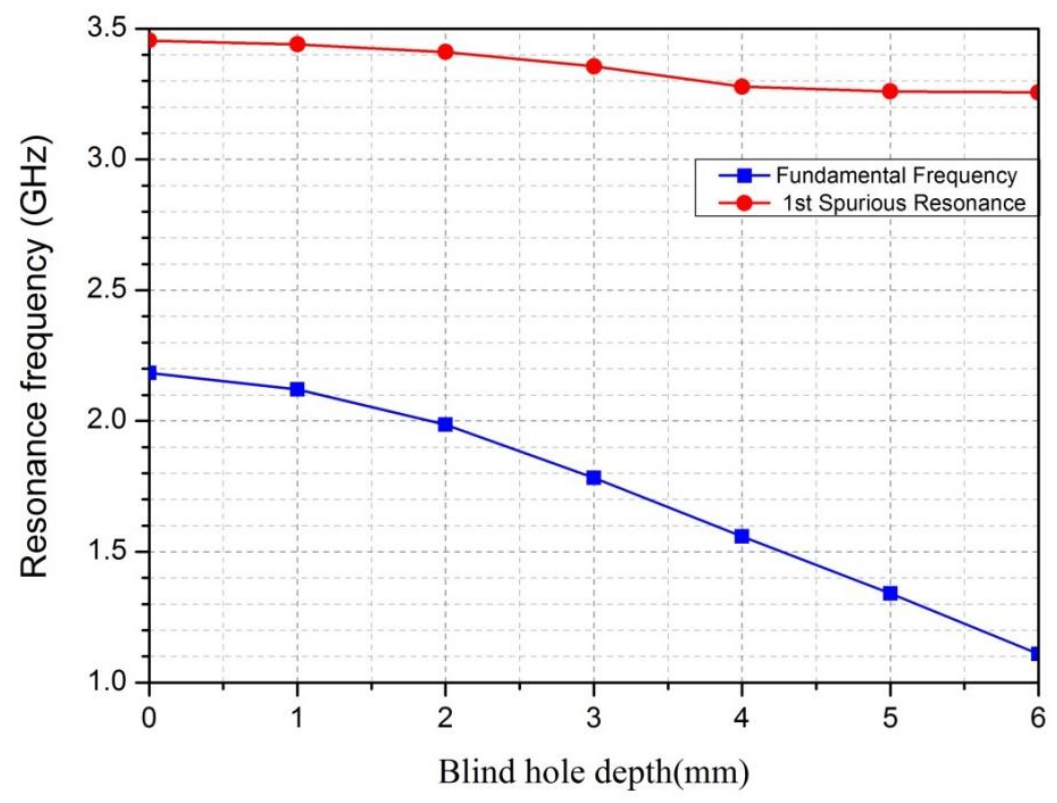

Figure 8.9: Simulated ceramic rectangular waveguide fundamental and first spurious frequency variation by placing the metal coated hole at the centre of broad wall of it.

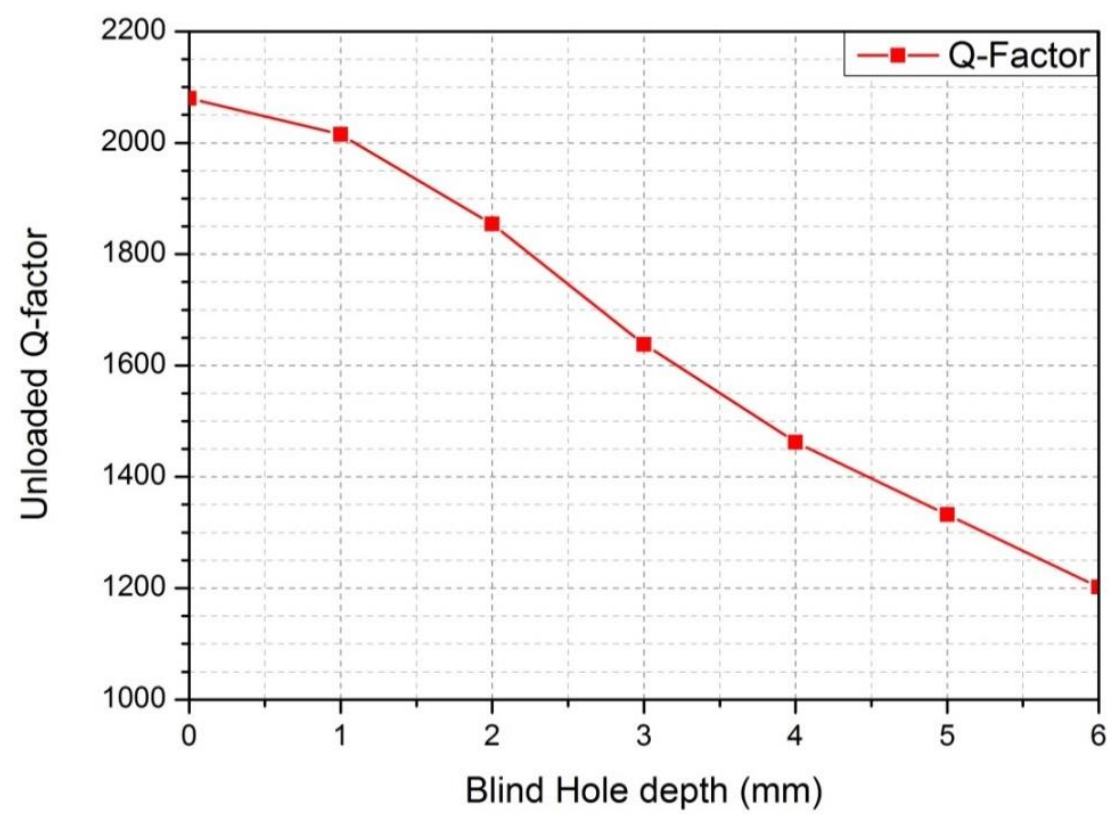

Figure 8.10: Simulated ceramic rectangular waveguide unloaded Q-factor variation by placing the metal coated hole at the centre of broad wall of it. 
Resonator volume can be reduced further by placing the blind hole at its centre at the expense of the reduced Q-factor. Alternatively only first and last resonators in the existing design can be replaced by the improved spurious low $\mathrm{Q}$ resonators without effecting the selectivity of the filter very much [91].

\subsection{Surface roughness}

The electric field and the power of a wave decrease exponentially when it penetrates into the surface of the conductor. At a depth, where electric field intensity is attenuated 1/e times of the value it has at the surface of the conductor and the power is decreased to $1 / \mathrm{e}^{2}$ times of its initial value is called skin depth of the conductor [60]. The skin depth of the conductor can be calculated as [92]

$$
\delta=\sqrt{\frac{\rho}{\pi f \mu}}
$$

Where $\rho$ is the resistivity of the conductor in $\Omega \mathrm{m}$ and frequency in Hz. At a depth of $5 \delta$ the power transported by the wave remains negligible as compared to the wave carrying power at the surface of the conductor [60]. If $\alpha_{c}$ is the conductor loss of surface metal then attenuation due to surface roughness of the conductor can be approximated from [93] as

$$
\propto_{c}^{\prime}=\propto_{c}\left[1+\frac{2}{\pi} \tan ^{-1}\left[1.4\left(\frac{\Delta}{\delta}\right)^{2}\right]\right]
$$

Where $\Delta$ representd surface roughness and $\delta$ is the skin depth of the metal at centre frequency of the filter. The RMS surface finish of ceramic waveguide filter was $0.5 \mu \mathrm{m}$ i.e. less than a skin depth of the conductor. Therefore, surface roughness does not contribute much to the losses of the filter. Figure 8.11 shows a HFSS ${ }^{\mathrm{TM}}$ simulation of Q-degradation of a ceramic waveguide resonator with the increased surface roughness of the metal plating at a centre frequency of $1.73 \mathrm{GHz}$. Figure 8.11 suggests that a surface roughness of less than $2 \mu \mathrm{m}$ does not degrade much of the Q-factor of the resonator. Therefore, it is good to have slight roughness in the surface as it helps to stick the metal to the surface of the resonator. 


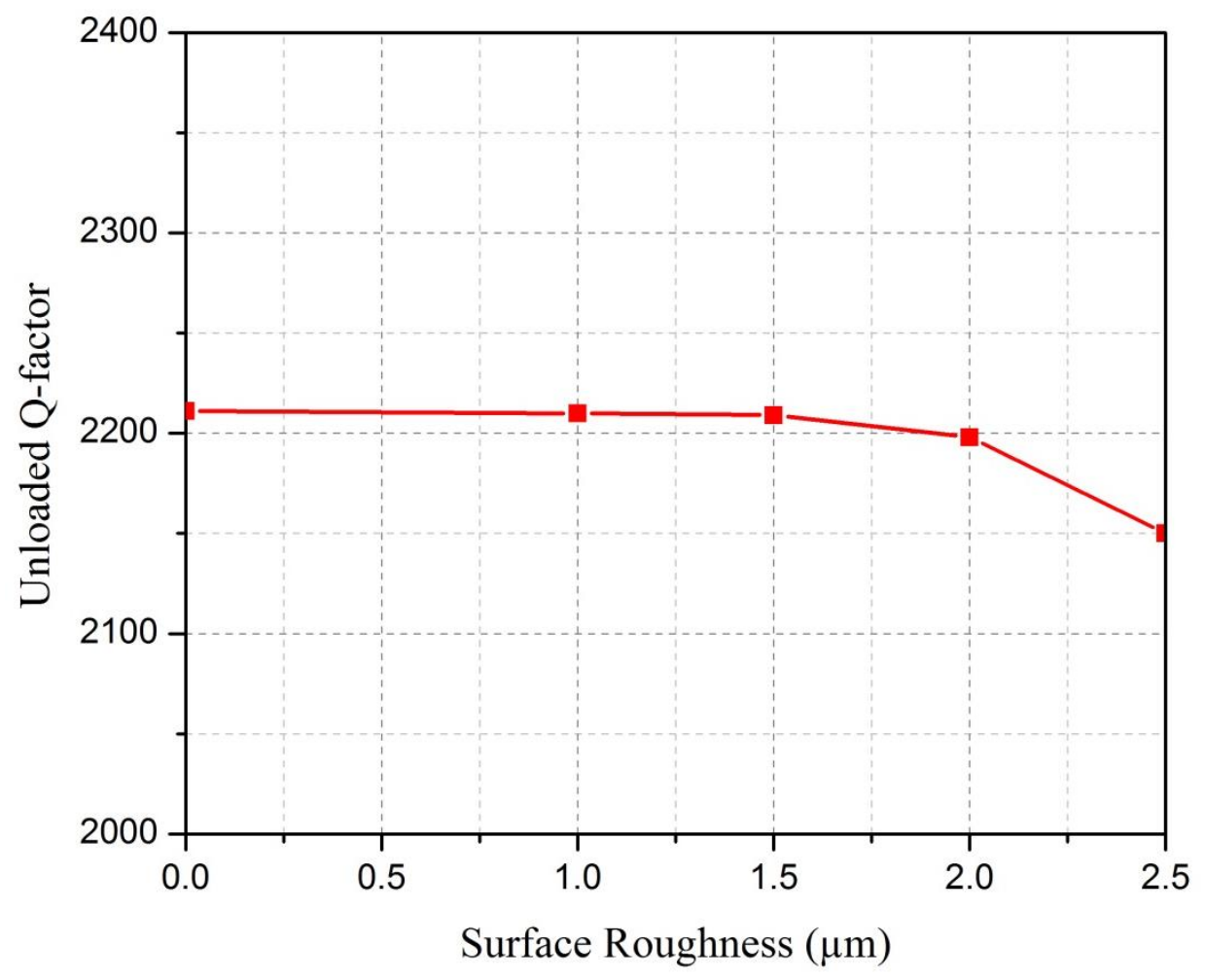

Figure 8.11: Simulated unloaded Q degradation with surface roughness

\subsection{Test fixture}

The initial design of the ceramic waveguide filter encountered some radiation losses due to leakage at the input and output terminals. This is being corrected in all other designs by making a test fixture to hold the ceramic filter tight against the external coupling probes. The copper pins of exact depth and diameter are fixed in the I/O terminals of the ceramic waveguide then the filter is placed in the test fixture as shown in Figure 8.13. The coaxial connectors are fixed at the bottom of the test fixture, there exists a spring between the fixture contact terminals and the waveguide I/O pins. The clips present in the test fixture are used to push the waveguide further down and hold it tightly so as to avoid any mismatch between I/O pins and coaxial connectors. In this way any leakage from $\mathrm{I} / \mathrm{O}$ pins is avoided to minimise the passband insertion loss of the filter. Figure 8.12 shows the generalized Chebyshev RX filter with input/output pins fixed in it. The measured results for this filter are discussed in Chapter 5. 


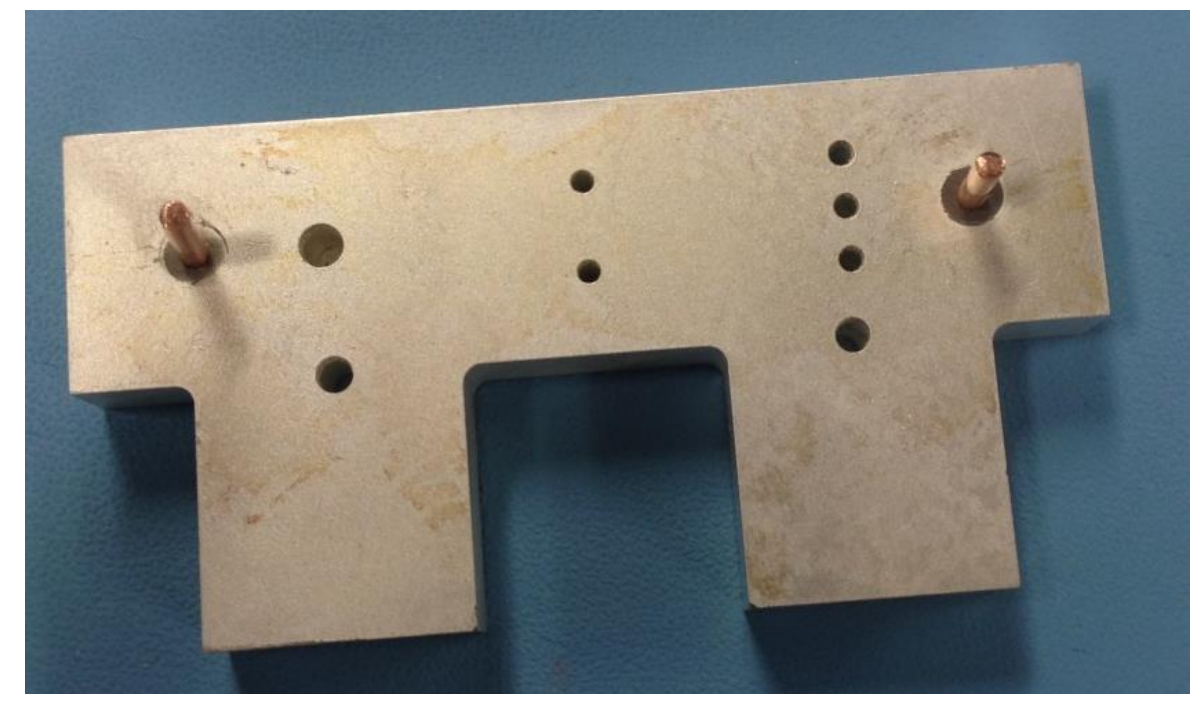

Figure 8.12: Fabricated RX filter with $\mathrm{I} / \mathrm{O}$ pins

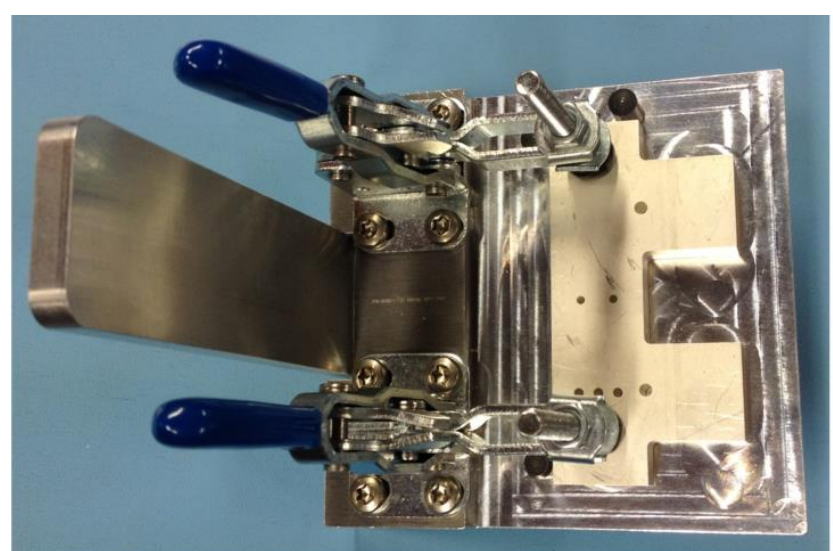

(a)

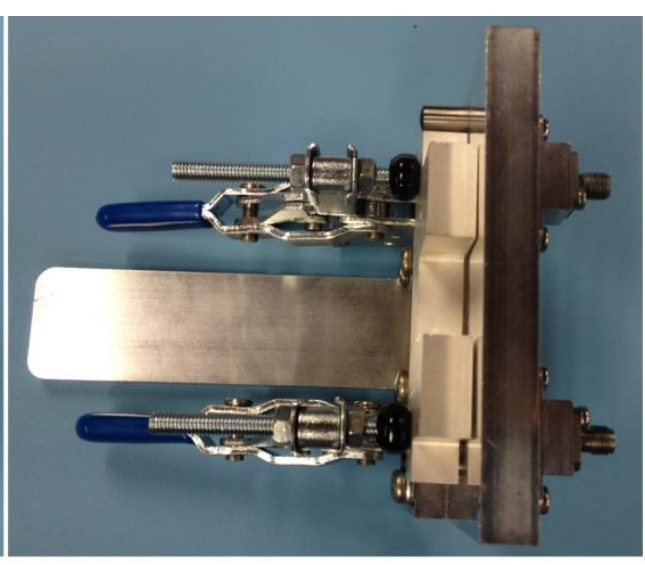

(b)

Figure 8.13: Test fixture with ceramic filter (a) Top view (b) Side view 


\section{CONCLUSIONS}

The motive behind this research is to reduce the volume and mass of the microwave filters used in the RF front end of cellular base stations without compromising on their electrical performance. Microwave filters/diplexers constitute the major portion of the total volume of the RF front end transceiver. Conventionally, TEM coaxial resonator filters or waveguide cavity filters are used in a cellular base station with the stringent electrical performance to avoid interference in ever increasing congested radio spectrum. In this thesis, compact and miniaturized integrated ceramic waveguide resonators are designed to replace the existing bulky coaxial or waveguide cavity resonators used in the microwave filters. Waveguide and TM mode ceramic resonators offer very high Q-factors but they are bulky. When a waveguide cavity resonator is loaded with a ceramic material of permittivity $\varepsilon_{r}$ its physical dimensions reduce by a factor $\frac{1}{\sqrt{\varepsilon_{r}}}$, therefore a significant volume reduction can be achieved by loading the waveguide resonator with a ceramic material of high permittivity. Although the Q-factor of the resonator is also degraded with the same proportion of $\frac{1}{\sqrt{\varepsilon_{r}}}$, but still an acceptable Q-factor can be maintained in a significant smaller volume as compared to coaxial resonators. The Q-factor, the fundamental resonance and spurious resonances of a metal coated ceramic rectangular block are calculated using classical waveguide equations and are verified through EM simulation tool $\mathrm{HFSS}^{\mathrm{TM}}$. The exterior of the rectangular ceramic block is coated with a silver ink of conductivity $4.4 \times 10^{7} \mathrm{~s} / \mathrm{m}$, which makes it a dielectric loaded waveguide resonator. The ceramic rectangular waveguide resonator offers wide spurious free bandwidth at the DCS uplink frequency band which makes it 
suitable to design microwave filters used in cellular base station front end.

A Chebyshev and a generalized Chebyshev integrated ceramic rectangular waveguide resonator filter are designed in this thesis. The insertion loss method of microwave filter design is used to realize these filters. All positive inter resonator couplings are achieved by placing through holes in the broad wall of the ceramic waveguide. These through holes behave as impedance inverters over a broad bandwidth of the filter. The amount of coupling bandwidth required is controlled by the diameter of the through holes and their offset from the side walls. The generalized Chebyshev ceramic waveguide filter consists of two cross coupled triplets in order to realize the transmission zeros at the both sides of the passband of the filter. The inductive couplings are realized by through holes behaving as shunt inductors between half wavelength ceramic waveguide resonators. The capacitive coupling required to form a capacitive triplet to produce a transmission zero at the lower side of the passband is achieved by placing a metal coated blind hole in the broad wall of the waveguide between half wavelength ceramic waveguide resonators. Due to the resonating nature of the blind hole an extra transmission zero is achieved. A further design of generalized Chebyshev filter is fabricated with tuning screws to compensate any material discrepancies or mechanical tolerances. The metal tuning screws are placed at the high E-field region of the ceramic waveguide resonators.

An integrated ceramic rectangular waveguide diplexer is designed after designing two individual Chebyshev and generalized Chebyshev filters constituting the TX and RX branches of the diplexer. Both filters are combined in a parallel configuration with the help of a common junction. The common junction is an extra piece of ceramic which sits in between both filters. The complete structure of the diplexer consists of a metal coated ceramic block with various through and blind holes to realize different inter resonator and external couplings. All of the filters and diplexer are excited using coaxial probes placed at the centre of the broad wall of the external resonators in each individual design. The coaxial probe used at the common junction of the diplexer is offset from the centre of the waveguide broad wall in order to achieve wideband coupling bandwidth. A test fixture is designed to avoid any leakage at the input terminals of the waveguide. 
An integrated ceramic waveguide low pass filter is designed to be used with bandpass waveguide filters to provide a very wide spurious free bandwidth. The stopband of the low pass ceramic waveguide filter can be further enhanced by varying the height or length of the high impedance sections of the waveguide.

Finally different practical issues related to integrated ceramic waveguide filter and diplexer design are discussed. These resonators can be fine-tuned using ceramic or metal tuning screw placed at the centre of the broad wall of the waveguide resonator. The surface finish of the ceramic waveguide filters was less than a skin depth i.e. < $0.5 \mu \mathrm{m}$, which does not contribute much to the conductor losses of the resonator. If a significant gap is maintained between tuning screw and the resonator then these integrated ceramic waveguide filters can be used for high power applications.

\subsection{Future Work}

The work presented in this thesis is limited to single mode ceramic waveguide filter and diplexer design. It can be extended in several aspects. The volume of the filters presented here can be further improved significantly by designing integrated ceramic waveguide filters composed of dual or triple mode ceramic resonators. Dual passband filters are another possible extension of this work.

Also different techniques can be used to improve the spurious performance of the single mode ceramic waveguide resonator. This can be achieved by introducing a metal coated circular hole at the centre of the ceramic waveguide resonator making it TEM resonator. This decreases the resonant frequency of the resonator below cuttoff and also decreases the Q-factor and size of the resonator. A possible configuration could be to use these lower Q TEM ceramic resonators with single mode ceramic resonators to improve overall spurious performance of the filter without compromising on its selectivity.

Another possible extension is the investigation of an integrated device composed of a ceramic waveguide diplexer and low pass corrugated ceramic waveguide filter for better spurious performance which could further be extended to achieve an integration of antenna with the ceramic waveguide diplexer.

The concept of frequency dependent coupling can be investigated to design an inline generalised Chebyshev integrated ceramic waveguide filter using variable height 
discontinuities/blind holes for inter resonator couplings. Each blind hole resonates at a frequency in the stopband of the filter thus producing a transmission zero.

It was difficult to machine a deep narrow blind hole to achieve negative coupling between resonators in generalised Chebyshev filter. Also the blind hole re-resonates at a higher frequency; if the diameter of the hole is not selected properly it can resonate near the passband and can degrade the return loss performance in the passband. Therefore, to avoid any blind hole in the design, all inductive ceramic filter can be designed by doubling the length of second resonator in a triplet which introduces a $90^{\circ}$ phase shift. Therefore, transmission zero is moved to the other side of the passband. 


\section{REFRENCES}

[1] C. Kudsia, R. Cameron, and T. Wai-Cheung, "Innovations in microwave filters and multiplexing networks for communications satellite systems," Microwave Theory and Techniques, IEEE Transactions on, vol. 40, pp. 1133-1149, 1992.

[2] R. V. Snyder, "Evolution of passive and active microwave filters," in Microwave Symposium Digest (MTT), 2012 IEEE MTT-S International, 2012, pp. 1-3.

[3] I. Hunter, Ed., Theory and design of microwave filters. London: The institute of electrical engineers, 2001.

[4] R. J. Cameron, C. M. Kudsia, and R. R. Mansour, Microwave filters for communication systems: fundamentals, design, and applications: WileyInterscience, 2007.

[5] R. Levy and P. Petre, "Design of CT and CQ filters using approximation and optimization," Microwave Theory and Techniques, IEEE Transactions on, vol. 49, pp. 2350-2356, 2001.

[6] R. J. Cameron, "General coupling matrix synthesis methods for Chebyshev filtering functions," Microwave Theory and Techniques, IEEE Transactions on, vol. 47, pp. 433-442, 1999.

[7] D. M. Pozar, Microwave engineering: Wiley, 1997.

[8] S. B. Cohn, "Direct-Coupled-Resonator Filters," Proceedings of the IRE, vol. 45, pp. 187-196, 1957.

[9] V. E. Boria, P. Soto, and S. Cogollos, "Distributed Models for Filter Synthesis," Microwave Magazine, IEEE, vol. 12, pp. 87-100, 2011.

[10] J. D. Rhodes, Theory of Electrical Filters: John Wiley \& Sons Canada, Limited, 1976.

[11] S. F. Peik and R. R. Mansour, "A novel design approach for microwave planar filters," in Microwave Symposium Digest, 2002 IEEE MTT-S International, 2002, pp. 1109-1112 vol. 2.

[12] S. Bila, D. Baillargeat, M. Aubourg, S. Verdeyme, P. Guillon, F. Seyfert, J. Grimm, L. Baratchart, C. Zanchi, and J. Sombrin, "Direct electromagnetic optimization of microwave filters," Microwave Magazine, IEEE, vol. 2, pp. 4651, 2001.

[13] P. Kozakowski and M. Mrozowski, "Automated CAD of coupled resonator filters," Microwave and Wireless Components Letters, IEEE, vol. 12, pp. 470472, 2002.

[14] J. V. M. Ros, P. S. Pacheco, H. E. Gonzalez, V. E. B. Esbert, C. B. Martin, M. T. Calduch, S. C. Borras, and B. G. Martinez, "Fast automated design of waveguide filters using aggressive space mapping with a new segmentation strategy and a hybrid optimization algorithm," Microwave Theory and Techniques, IEEE Transactions on, vol. 53, pp. 1130-1142, 2005.

[15] C. E. Chrisostomidis, M. Guglielmi, P. Young, and S. Lucyszyn, "Application of Chained Functions to Low-Cost Microwave Band-pass Filters Using Standard PCB Etching Techniques," in Microwave Conference, 2000. 30th European, 2000, pp. 1-4.

[16] C. E. Chrisostomidis and S. Lucyszyn, "On the theory of chained-function filters," Microwave Theory and Techniques, IEEE Transactions on, vol. 53, pp. 3142-3151, 2005.

[17] M. Guglielmi and G. Connor, "Chained function filters," Microwave and Guided 
Wave Letters, IEEE, vol. 7, pp. 390-392, 1997.

[18] V. V. Tyurnev, "Coupling coefficients of resonators in microwave filter theory," Progress In Electromagnetics Research B, vol. 21, pp. 47-67, 2010.

[19] J. Helszajn and I. o. E. Engineers, Ridge Waveguides and Passive Microwave Components: Institution of Engineering and Technology, 2000.

[20] G. L. Matthaei, L.Young and E. M. T. Jones, Microwave filters, impedancematching networks, and coupling structures: McGraw-Hill, 1980.

[21] J. B. Thomas, "Cross-coupling in coaxial cavity filters - a tutorial overview," Microwave Theory and Techniques, IEEE Transactions on, vol. 51, pp. 13681376, 2003.

[22] R. J. Cameron, "Advanced coupling matrix synthesis techniques for microwave filters," Microwave Theory and Techniques, IEEE Transactions on, vol. 51, pp. $1-10,2003$.

[23] D. Swanson and G. Macchiarella, "Microwave filter design by synthesis and optimization," Microwave Magazine, IEEE, vol. 8, pp. 55-69, 2007.

[24] W. Chi, K. A. Zaki, A. E. Atia, and T. G. Dolan, "Dielectric combline resonators and filters," Microwave Theory and Techniques, IEEE Transactions on, vol. 46, pp. 2501-2506, 1998.

[25] R. Levy, R. V. Snyder, and G. Matthaei, "Design of microwave filters," Microwave Theory and Techniques, IEEE Transactions on, vol. 50, pp. 783-793, 2002.

[26] S. B. Cohn, "Microwave Bandpass Filters Containing High-Q Dielectric Resonators," Microwave Theory and Techniques, IEEE Transactions on, vol. 16, pp. 218-227, 1968.

[27] R. R. Mansour, "Filter technologies for wireless base stations," Microwave Magazine, IEEE, vol. 5, pp. 68-74, 2004.

[28] I. C. Hunter, L. Billonet, B. Jarry, and P. Guillon, "Microwave filtersapplications and technology," Microwave Theory and Techniques, IEEE Transactions on, vol. 50, pp. 794-805, 2002.

[29] N. E. A. Rashid, M. T. Ali, and N. Hamzah, "Properties of dielectric combline resonator for base station filters," in RF and Microwave Conference, 2004. RFM 2004. Proceedings, 2004, pp. 76-79.

[30] T. Nishikawa, "Microwave Ceramic Dielectrics and Their Applications," in Microwave Conference, 1988. 18th European, 1988, pp. 70-80.

[31] Y. Konishi, "Novel dielectric waveguide components-microwave applications of new ceramic materials," Proceedings of the IEEE, vol. 79, pp. 726-740, 1991.

[32] W. Chi and K. A. Zaki, "Dielectric resonators and filters," Microwave Magazine, IEEE, vol. 8, pp. 115-127, 2007.

[33] A. Knack, J. Mazierska, and H. Piel, "Dielectric Resonator Filters for UMTS Systems," in Art of Miniaturizing RF and Microwave Passive Components, 2008. IMWS 2008. IEEE MTT-S International Microwave Workshop Series on, 2008, pp. 30-33.

[34] Z. Zhang, C. Chen, and X. Wu, "A compact cavity filter with novel TM mode dielectric resonator structure," in Microwave Technology \& Computational Electromagnetics (ICMTCE), 2011 IEEE International Conference on, 2011, pp. 111-113.

[35] K. Pance, "Multiband tunable dielectric resonator filters," in Radio and Wireless Symposium, 2006 IEEE, 2006, pp. 395-398.

[36] M. G. B. A. IOACHIM, L. NEDELCU, C. A. DUȚU, "Band-pass filters with (Zr0.8, Sn0.2)TiO4 dielectric resonators," JOURNAL OF OPTOELECTRONICS 
AND ADVANCED MATERIALS, vol. 8, pp. 941-943, 2006.

[37] G.-C. L. Ji-Fuh Liang, Marco Song, George He, and Tony An. (2011-06-17) Single Mode DR Filters for Wireless Base Stations. ZTE Communications.

[38] H. Salehi, R. K. Reddy, T. Lukkarila, and S. Amir, "Spurious suppression of dielectric filters in practical wireless systems," in Microwave Symposium Digest, 2008 IEEE MTT-S International, 2008, pp. 1087-1090.

[39] K. Pance, "Base Station Filters without Irises," in Microwave Symposium Digest, 2006. IEEE MTT-S International, 2006, pp. 238-241.

[40] I. C. Hunter, J. D. Rhodes, and V. Dassonville, "Dual-mode filters with conductor-loaded dielectric resonators," Microwave Theory and Techniques, IEEE Transactions on, vol. 47, pp. 2304-2311, 1999.

[41] J. M. Chuma and D. Mirshekar-Syahkal, "Compact Dielectric Loaded Combline Filter with Low Insertion-loss," in Microwave Conference, 2000. 30th European, 2000, pp. 1-4.

[42] M. Esa, M. F. M. Yusoff, N. A. Murad, and R. Sinnathamby, "C-band directcoupled cavity waveguide filter design using MathCAD software," in Applied Electromagnetics, 2005. APACE 2005. Asia-Pacific Conference on, 2005, p. 4 pp.

[43] M. Latif, F. Shafiq, M. Q. Shafique, and N. Naseer, "Design \& realization of direct coupled $\mathrm{Ku}$-band waveguide iris filter using coupling matrix model," in Applied Sciences and Technology (IBCAST), 2012 9th International Bhurban Conference on, 2012, pp. 363-367.

[44] S. Choocadee and S. Akatimagool, "Design and implementation of band pass filters in waveguide using simulation tools," in Electrical Engineering/Electronics, Computer, Telecommunications and Information Technology (ECTI-CON), 2011 8th International Conference on, 2011, pp. 248251.

[45] M. T. Sebastian, Dielectric materials for wireless communication. Elsevier Science \& Technology Books, 2008

[46] A. Abdelmonem, L. Ji-Fuh, Y. Hui-Wen, and K. A. Zaki, "Full-wave design of spurious free DR TE mode band pass filters," Microwave Theory and Techniques, IEEE Transactions on, vol. 43, pp. 744-752, 1995.

[47] S. J. Fiedziuszko, I. C. Hunter, T. Itoh, Y. Kobayashi, T. Nishikawa, S. N. Stitzer, and K. Wakino, "Dielectric materials, devices, and circuits," Microwave Theory and Techniques, IEEE Transactions on, vol. 50, pp. 706-720, 2002.

[48] B. H. G. a. M. Khalaj-Amirhosseini, "Compact band pass filters utilizing dielectric filled waveguides," Progress In Electromagnetics Research B, vol. 7, pp. 105-115, 2008.

[49] J. Hayashi and Y. Nikawa, "Waveguide filter using LTCC," in Microwave Conference, 2001. APMC 2001. 2001 Asia-Pacific, 2001, pp. 1012-1015 vol.3.

[50] S. Lucyszyn, D. Budimir, Q. H. Wang, and I. D. Robertson, "Design of compact monolithic dielectric-filled metal-pipe rectangular waveguides for millimetrewave applications," Microwaves, Antennas and Propagation, IEE Proceedings, vol. 143, pp. 451-453, 1996.

[51] M. S. Aftanasar, P. R. Young, I. D. Robertson, and S. Lucyszyn, "Fabrication of dielectric-filled rectangular waveguide using thick-film processing," in High Frequency Postgraduate Student Colloquium, 2001. 6th IEEE, 2001, pp. 82-87.

[52] L. Chen, Microwave electronics: measurement and materials characterization: John Wiley, 2004.

[53] S. Ramo, J. R. Whinnery, and T. Van Duzer, Fields and Waves in 
Communication Electronics: John Wiley \& Sons, 1994.

[54] P. S. Neelakanta, Handbook of Electromagnetic Materials: Monolithic and Composite Versions and Their Applications: Taylor \& Francis, 1995.

[55] D. Kajfez and P. Guillon, Dielelectric Resonators: Noble Publishing Corporation, 1998.

[56] H. J. Reich, H. L. Krauss, and P. F. Ordung, Microwave Theory and Techniques: Literary Licensing, LLC, 2013.

[57] P. N. Martin, J., "Coupling Bandwidth and Reflected Group Delay Characterization of Microwave Bandpass Filters," APPLIED MICROWAVE AND WIRELESS, vol. 11, 1999.

[58] T. Saad, Microwave Engineer's Handbook: Artech House, 1971.

[59] J. F. Liang, H. C. Chang, and K. A. Zaki, "Coaxial probe modeling in waveguides and cavities," Microwave Theory and Techniques, IEEE Transactions on, vol. 40, pp. 2172-2180, 1992.

[60] H. J. Reich, Microwave Theory and Techniques: Va Nostrand, 1953.

[61] R. M. Kurzrok, "General Three-Resonator Filters in Waveguide," Microwave Theory and Techniques, IEEE Transactions on, vol. 14, pp. 46-47, 1966.

[62] R. M. Kurzrok, "General Four-Resonator Filters at Microwave Frequencies (Correspondence)," Microwave Theory and Techniques, IEEE Transactions on, vol. 14, pp. 295-296, 1966.

[63] A. E. Atia, A. E. Williams, and R. W. Newcomb, "Narrow-band multiplecoupled cavity synthesis," Circuits and Systems, IEEE Transactions on, vol. 21, pp. 649-655, 1974.

[64] J. D. Rhodes and R. J. Cameron, "General Extracted Pole Synthesis Technique with Applications to Low-Loss TE<sub>011</sub> Mode Filters," Microwave Theory and Techniques, IEEE Transactions on, vol. 28, pp. 1018-1028, 1980.

[65] R. Sorrentino, L. Pelliccia, and S. Bastioli, "Recent progress in miniaturized and reconfigurable filters for advanced communications and space applications," in Microwaves, Radar and Remote Sensing Symposium (MRRS), 2011, 2011, pp. 20-23.

[66] I. C. Hunter, R. Ranson, A. Guyette, and A. Abunjaileh, "Microwave filter design from a systems perspective," Microwave Magazine, IEEE, vol. 8, pp. 7177, 2007.

[67] M. Dishal, "A Simple Design Procedure for Small Percentage Bandwidth Round-Rod Interdigital Filters (Correspondence)," Microwave Theory and Techniques, IEEE Transactions on, vol. 13, pp. 696-698, 1965.

[68] M. Dishal, "Alignment and Adjustment of Synchronously Tuned MultipleResonant-Circuit Filters," Proceedings of the IRE, vol. 39, pp. 1448-1455, 1951.

[69] N. Yildirim, O. A. Sen, and Y. Sen, "Synthesis of cascaded N-tuplet filters," in Telecommunications in Modern Satellite, Cable and Broadcasting Service, 2001. TELSIKS 2001. 5th International Conference on, 2001, pp. 153-162 vol.1.

[70] M. Politi and A. Fossati, "Direct coupled waveguide filters with generalized Chebyshev response by resonating coupling structures," in Microwave Conference (EuMC), 2010 European, 2010, pp. 966-969.

[71] L. Szydlowski, A. Lamecki, and M. Mrozowski, "Coupled-Resonator Waveguide Filter in Quadruplet Topology With Frequency-Dependent Coupling-A Design Based on Coupling Matrix," Microwave and Wireless Components Letters, IEEE, vol. 22, pp. 553-555, 2012.

[72] J. D. Rhodes, "Direct design of symmetrical interacting bandpass channel diplexers," Microwaves, Optics and Acoustics, IEE Journal on, vol. 1, pp. 34-40, 
1976.

[73] J. L. Haine and J. D. Rhodes, "Direct Design Formulas for Asymmetric Bandpass Channel Diplexers," Microwave Theory and Techniques, IEEE Transactions on, vol. 25, pp. 807-813, 1977.

[74] R. Levy, "Synthesis of non-contiguous diplexers using broadband matching theory," in Microwave Filters and Multiplexers, IEE Colloquium on, 1990, pp. 611-613.

[75] G. Macchiarella and S. Tamiazzo, "Novel Approach to the Synthesis of Microwave Diplexers," Microwave Theory and Techniques, IEEE Transactions on, vol. 54, pp. 4281-4290, 2006.

[76] D. Wolansky, J. Vorek, R. Tkadlec, F. Hrnico, "Virtual prototyping of diplexers by using CST Studio," in Microwave Techniques (COMITE), 2010 15th International Conference on, 2010, pp. 191-194.

[77] S. Sanghoon and S. Kanamaluru, "Diplexer design using EM and circuit simulation techniques," Microwave Magazine, IEEE, vol. 8, pp. 77-82, 2007.

[78] N. Marcuvitz and I. o. E. Engineers, Waveguide Handbook: McGraw-Hill, 1951.

[79] L. Ji-Fuh, H. C. Chang, and K. A. Zaki, "Coaxial probe modeling in waveguides and cavities," Microwave Theory and Techniques, IEEE Transactions on, vol. 40, pp. 2172-2180, 1992.

[80] R. B. Keam and A. G. Williamson, "Broadband design of coaxial line/rectangular waveguide probe transition," Microwaves, Antennas and Propagation, IEE Proceedings, vol. 141, pp. 53-58, 1994.

[81] M. Hajian, D. P. Tran, and L. P. Ligthart, "Modeling the transition between a coaxial line and a flat rectangular waveguide," in Antennas and Propagation, 1995., Ninth International Conference on (Conf. Publ. No. 407), 1995, pp. 269272 vol.1.

[82] R. Levy, "Tapered Corrugated Waveguide Low-pass Filters," Microwave Theory and Techniques, IEEE Transactions on, vol. 21, pp. 526-532, 1973.

[83] O. Ishida and H. M. Maruf, "Expansion of a continuous stop band of a corrugated waveguide by attenuation pole control," in Microwave Conference Proceedings (APMC), 2012 Asia-Pacific, 2012, pp. 571-573.

[84] L. Young and B. M. Schiffman, "New and improved types of waffle-iron filters," Electrical Engineers, Proceedings of the Institution of, vol. 110, pp. 1191-1198, 1963.

[85] O. Monerris, P. Soto, S. Cogollos, V. E. Boria, J. Gil, C. Vicente, and B. Gimeno, "Accurate circuit synthesis of low-pass corrugated waveguide filters," in Microwave Conference (EuMC), 2010 European, 2010, pp. 1237-1240.

[86] R. Serway and J. Jewett, Physics for Scientists and Engineers with Modern Physics: Cengage Learning, 2013.

[87] Y. Ming, "Power-handling capability for RF filters," Microwave Magazine, IEEE, vol. 8, pp. 88-97, 2007.

[88] W. Chi and K. A. Zaki, "Analysis of power handling capacity of band pass filters," in Microwave Symposium Digest, 2001 IEEE MTT-S International, 2001, pp. 1611-1614, vol.3.

[89] A. R. Harish and R. J. Cameron, "Peak voltage analysis in high power microwave filters," in Microwave Filters and Multiplexers (Ref. No. 2000/117), IEE Colloquium on, 2000, pp. 10/1-10/5.

[90] A. Sivadas, Y. Ming, and R. Cameron, "A simplified analysis for high power microwave bandpass filter structures," in Microwave Symposium Digest. 2000 IEEE MTT-S International, 2000, pp. 1771-1774 vol.3. 
[91] P. M. Iglesias and I. C. Hunter, "Non-uniform Q-factor distribution in microwave filters," in 7th European Microwave Integrated Circuits Conference (EuMIC), 2012, pp. 802-805.

[92] W. C. Johnson, Transmission lines and networks: McGraw-Hill, 1950.

[93] T. C. Edwards and B. Steer, Foundations of interconnect and microstrip design: John Wiley, 2000. 